This document is downloaded from DR-NTU (https://dr.ntu.edu.sg) Nanyang Technological University, Singapore.

Meta-heuristics for scheduling flexible job shop

problems with constraints

Gao, Kaizhou

2015

https://hdl.handle.net/10356/65649

https://doi.org/10.32657/10356/65649 

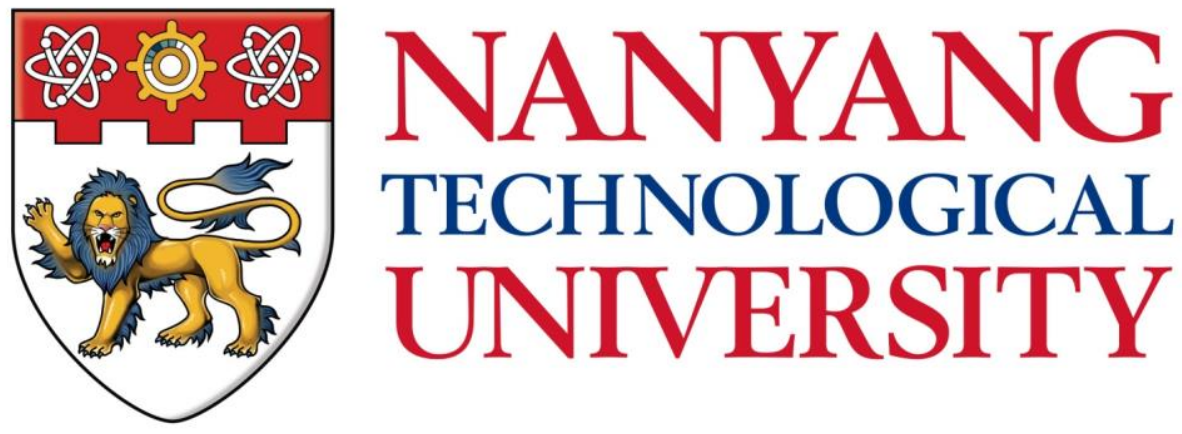

META-HEURISTICS FOR SCHEDULING FLEXIBLE JOB SHOP PROBLEMS WITH CONSTRAINTS

\author{
GAO KAIZHOU \\ SCHOOL OF ELECTRICAL AND ELECTRONIC ENGINEERING \\ 2015
}





\title{
META-HEURISTICS FOR SCHEDULING FLEXIBLE JOB SHOP PROBLEMS WITH CONSTRAINTS
}

\author{
GAO KAIZHOU
}

SCHOOL OF ELECTRICAL AND ELECTRONIC ENGINEERING

A thesis submitted to the Nanyang Technological University in fulfillment of the requirements for the degree of

Doctor of Philosophy 



\section{Statement of Originality}

I hereby certify that the content of this thesis is the result of work done by me and has not been submitted for a higher degree to any other University or Institution.

Date 



\section{Acknowledgments}

I would like to take this opportunity to express my gratitude to those who contributed to the successful completion of my postgraduate studies and this research thesis. I would thank them all, but there are some people who need special recognition.

First, I want to thank my supervisor, Associate Professor Ponnuthurai Nagaratnam Suganthan. He has provided his careful guidance, stimulating suggestions, exact insights and profound knowledge in supervising my research work throughout my $\mathrm{Ph} . \mathrm{D}$ study. Without his precise and appropriate instructions at every step during my research, I could not accomplish my postgraduate study smoothly.

Thank to my co-supervisor, Dr Chong Chin Soon, for advice, discussion and helping me in reviewing and modifying my papers. He is always prudent in every detail. I really appreciate all his generous help.

I also want to express my thanks to Dr. Pan Quan-Ke, Mr. Chua TayJin, and Mr. Cai TianXiang. During the collaboration with them on several research works, they have provided valuable suggestions as well as shared precious experiences on those work.

I would like to thank all my good friends and lab-mates including Mr. Yang ChengBin, my roommate, for the fun and joy we have had during the past three years.

Meanwhile, thanks a lot to my dear parents, my older brother, my sister in law, my nephews and nieces.

Finally, thanks with lots of love to my wife, Wang MeiFang, and my son, Gao MingYu. They encouraged and supported me to complete the Ph.D study with their patience and love. 


\section{Contents}

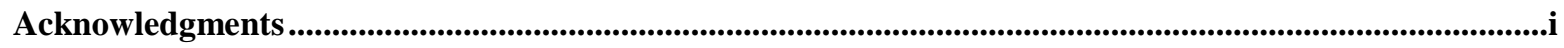

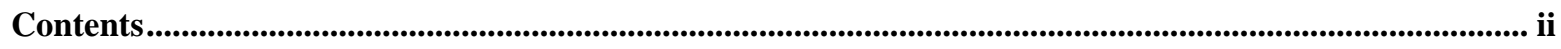

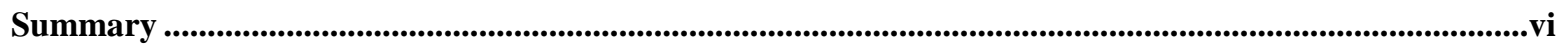

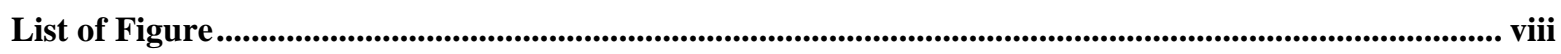

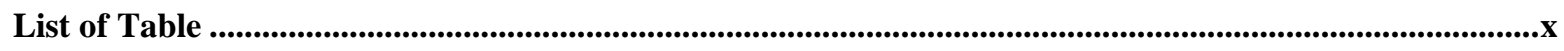

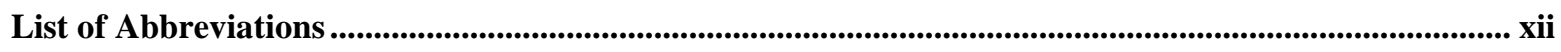

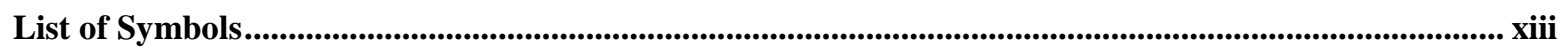

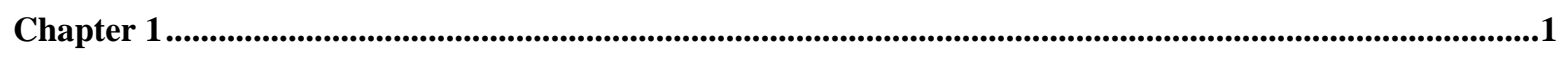

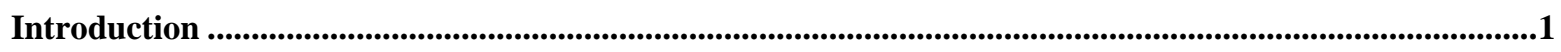

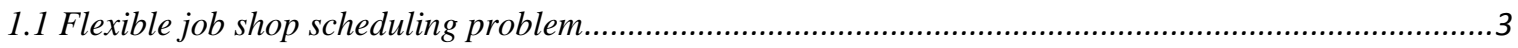

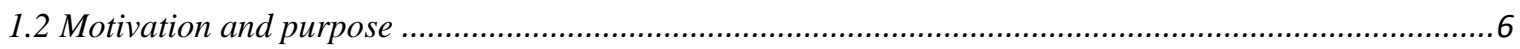

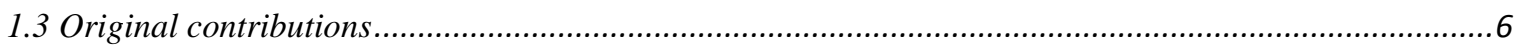

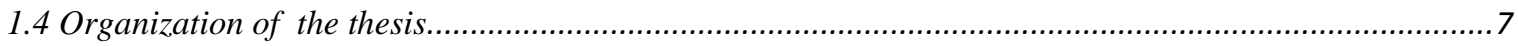

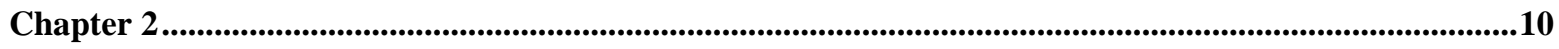

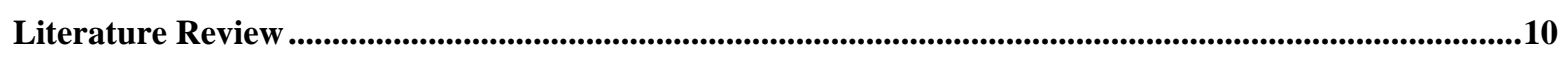

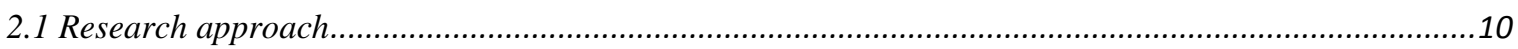

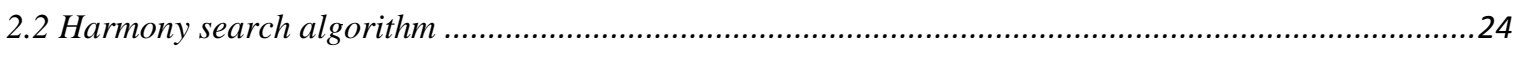

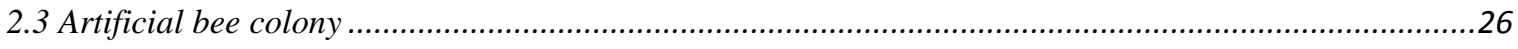

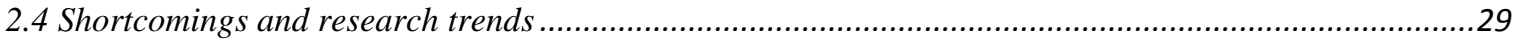

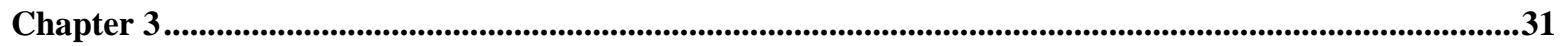

Modelling FJSSP with Multiple Constraints......................................................................................................31

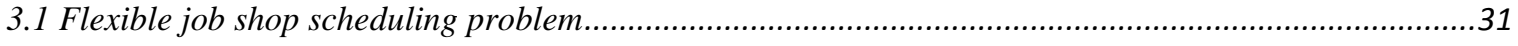

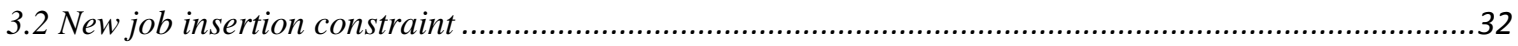

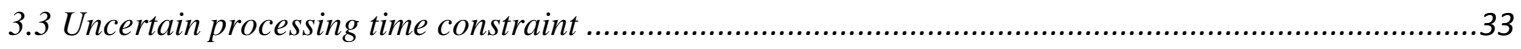

3.3.1 Modeling based on most probable processing time ............................................................33

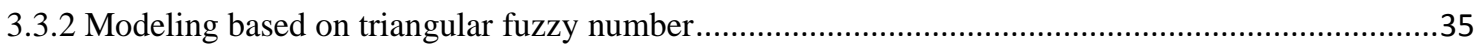

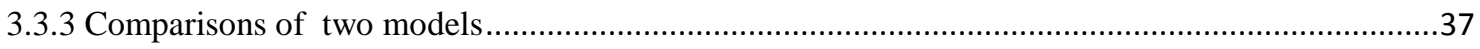

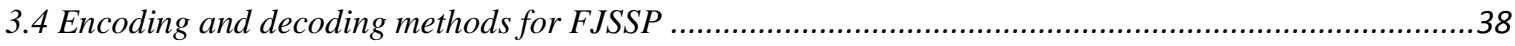

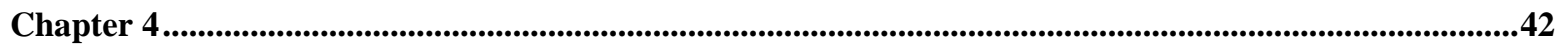

DHS Algorithm for FJSSP .............................................................................................................................42

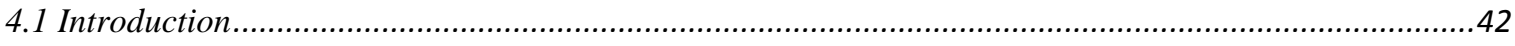

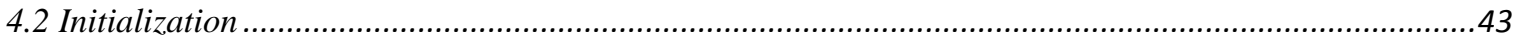




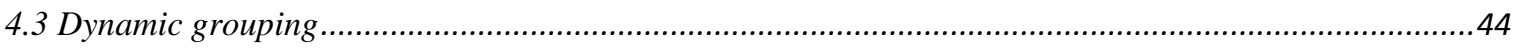

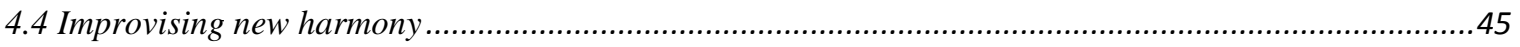

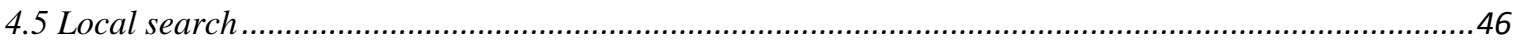

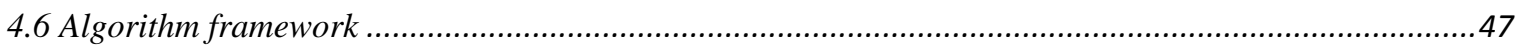

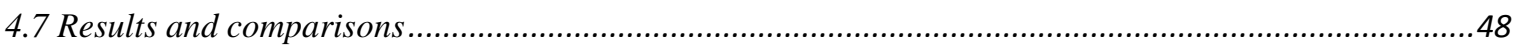

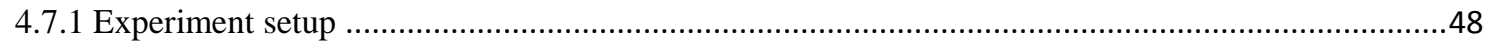

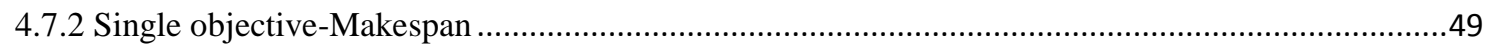

4.7.3 Pareto-based multi-objective objectives ..............................................................................50

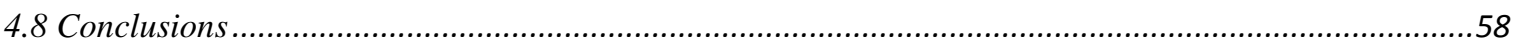

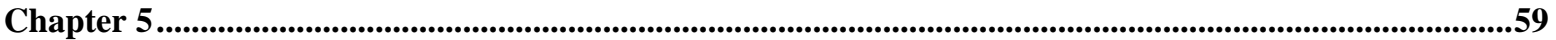

Two-stage ABC Algorithm for FJSSP with New Job Insertion..............................................................59

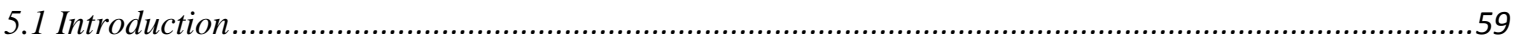

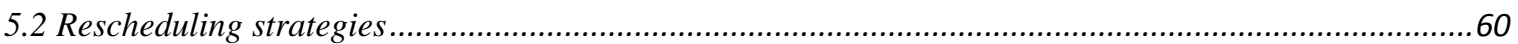

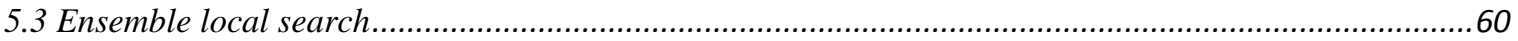

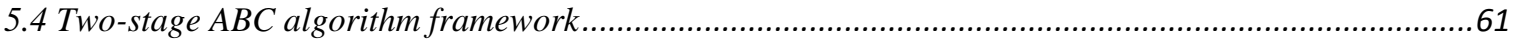

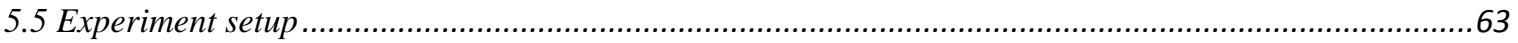

5.6 Discussion and comparison of three re-scheduling strategies ...........................................................64

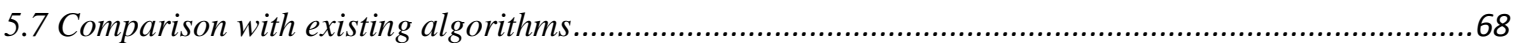

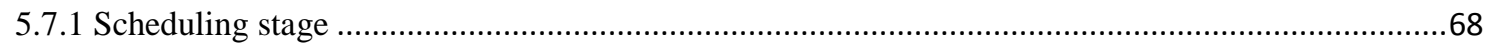

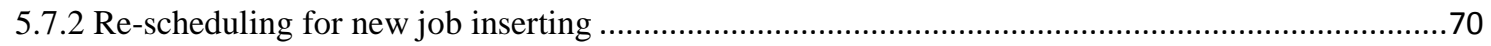

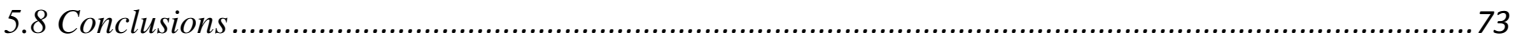

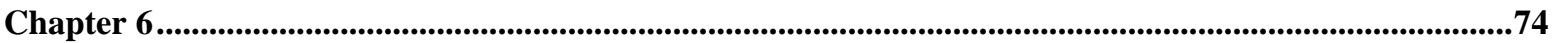

Two-stage DHS Algorithm for FJSSP with Multiple Constraints......................................................74

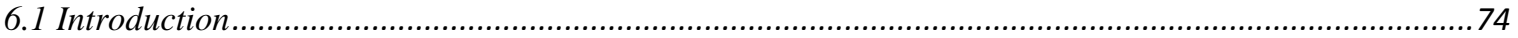

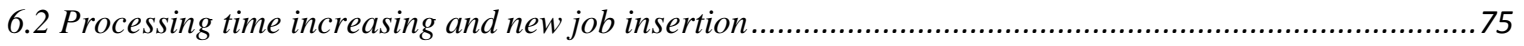

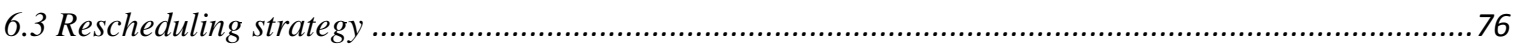

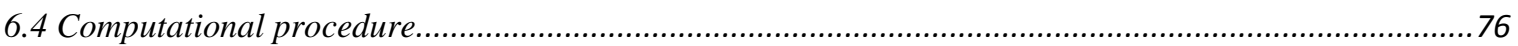

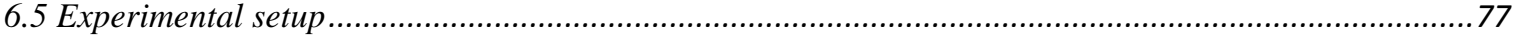

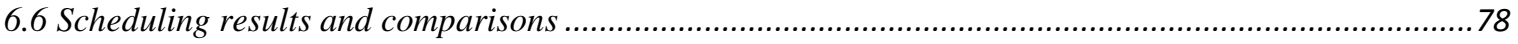

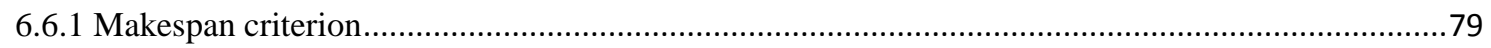

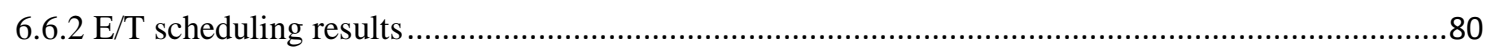

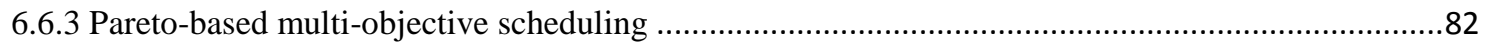

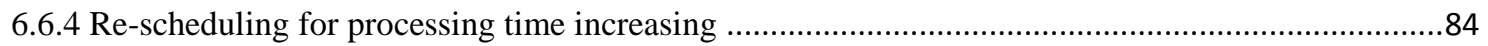

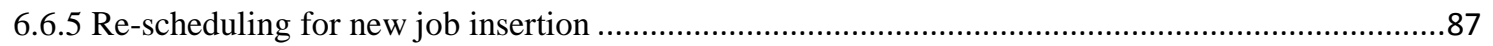

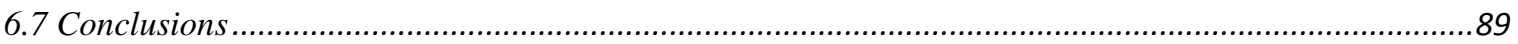

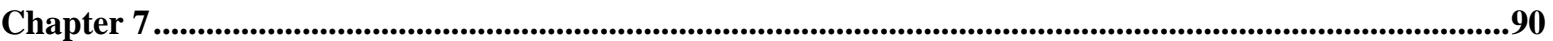


FDHS Algorithm for FJSSP with Fuzzy Processing Time ......................................................................................90

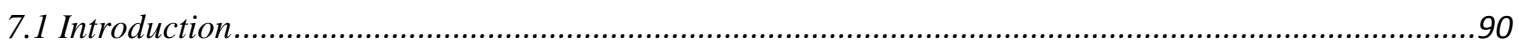

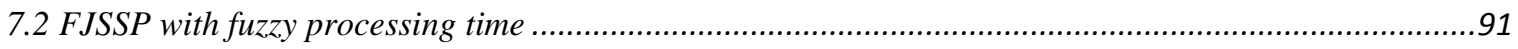

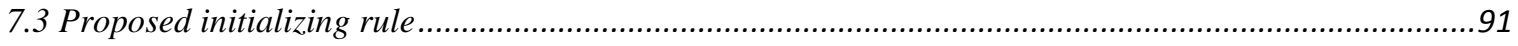

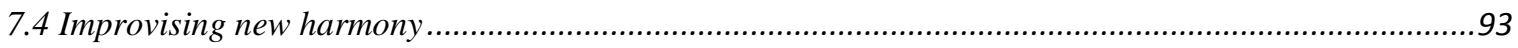

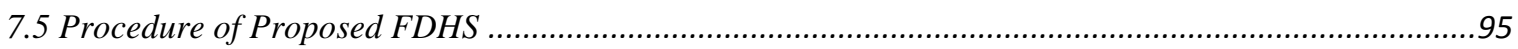

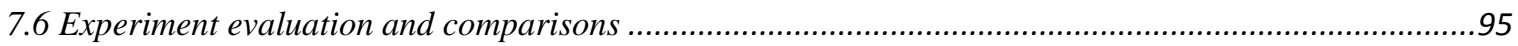

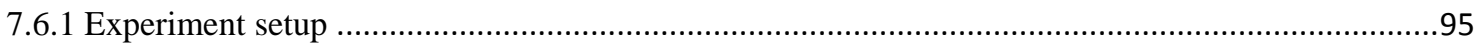

7.6.2 Performance of Proposed MinEnd Heuristic .........................................................................95

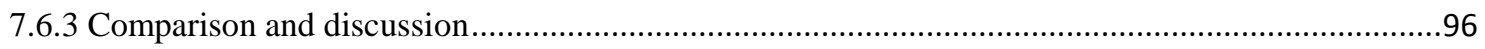

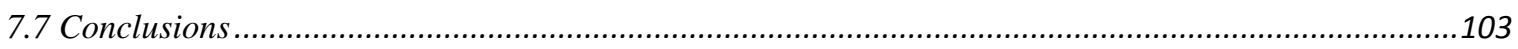

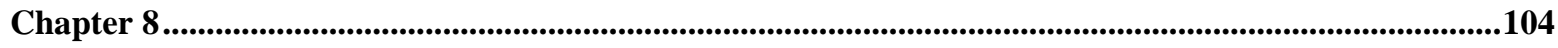

FABC Algorithm for Multi-objective FJSSP with Fuzzy Processing Time...............................................104

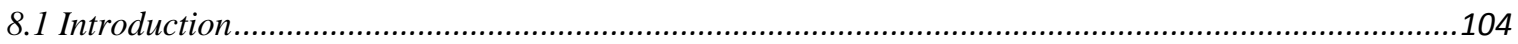

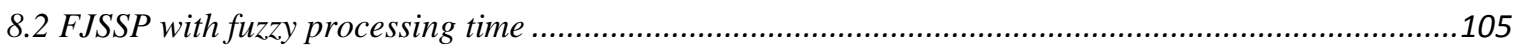

8.3 FABC for FJSSP with fuzzy processing time ...................................................................... 105

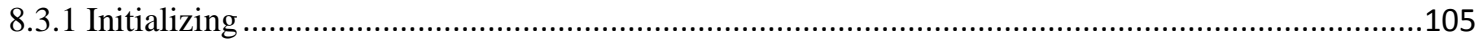

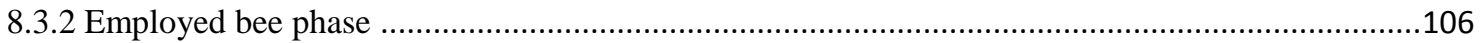

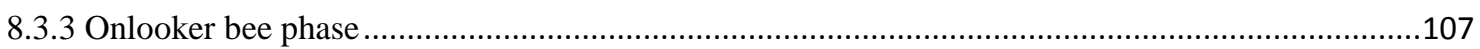

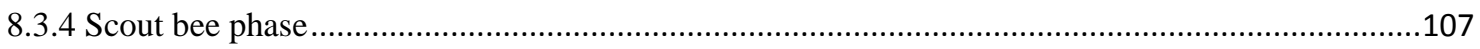

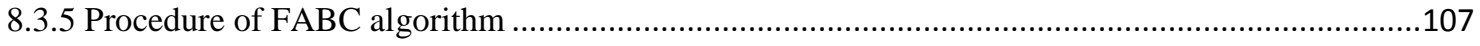

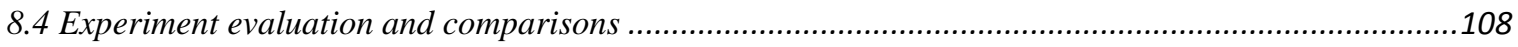

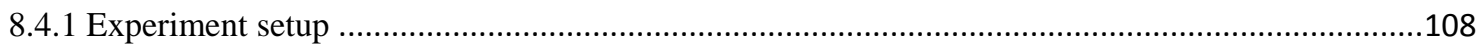

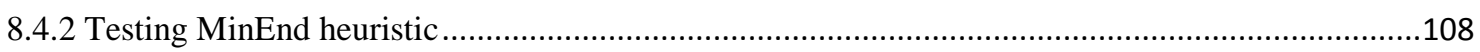

8.4.3 Maximum fuzzy completion time objective ..............................................................110

8.4.4 Maximum fuzzy machine workload objective .....................................................................117

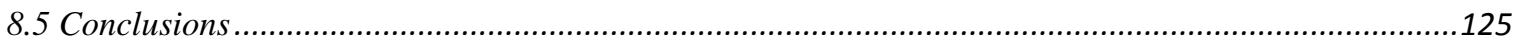

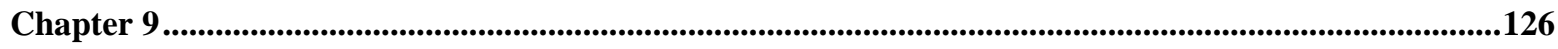

Two-stage FABC Algorithm for Multi-objective and Multi-constraint FJSSP .................................126

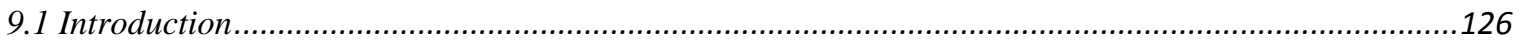

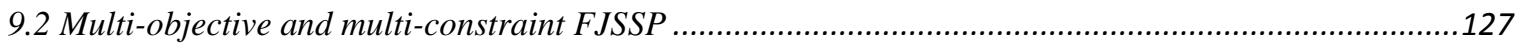

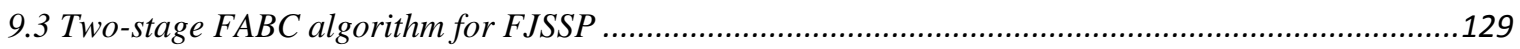

9.4 Experiment evaluation and comparisons ............................................................................130

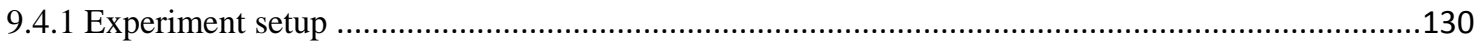

9.4.2 Maximum fuzzy completion time objective....................................................................131

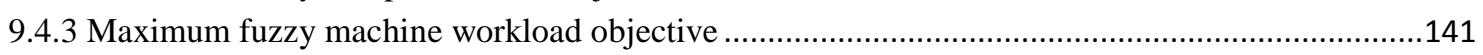

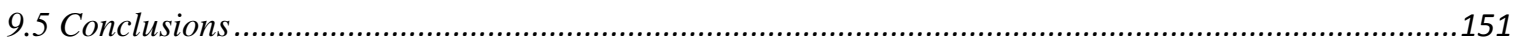




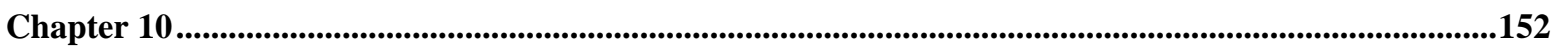

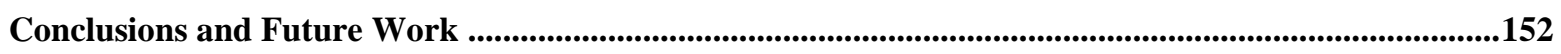

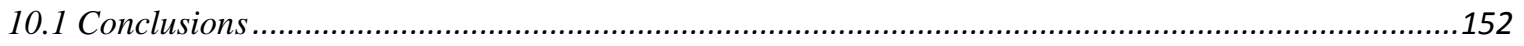

10.2 Future work

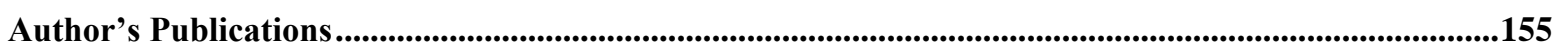

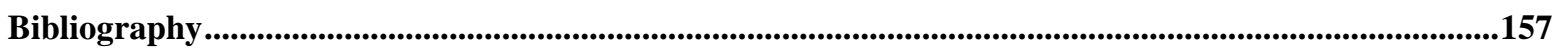

Appendix ...................................................................................................................................................................167

A. The benchmark instances used in this thesis...................................................................................167

B. 8 real instances with most probable processing time ……..................................................................178

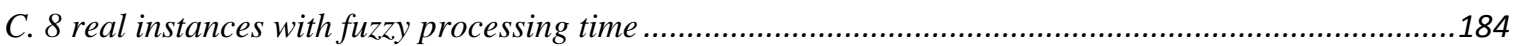




\section{Summary}

Flexible job-shop scheduling problem (FJSSP) is an extension of the classical job shop scheduling problem (JSSP) with practical applications. FJSSP has been proven as an NP-hard problem. Many researchers have focused on FJSSP in recent years.

FJSSP includes two sub-problems: 1. machine assignment that is to select a machine from a set of candidate machines for operations; 2 . operation sequencing that is to schedule the operations on machines to obtain a feasible solution. In the classical JSSP problem, one operation can be processed on only one machine. Hence, the FJSSP is more difficult than the classical JSSP. There is a great variety of real-world problems that can be modeled as FJSSP, e.g., optimization of crane operations, simulation and optimization of transport systems, and scheduling of manufacturing and remanufacturing systems.

In this thesis, the scheduling problems in the remanufacturing industry are modeled as FJSSP with multiple constraints. The constraints are new job insertion and uncertain processing time. The uncertain processing time constraint is built into two models, one is based on most probable processing time and another is based on fuzzy processing time. A rescheduling operator is executed when a new job is inserted or processing time becomes larger than the most probable processing time. The problem size may change dynamically during scheduling and rescheduling stages. The objectives include minimizing maximum completion time (Makespan), minimizing maximum machine workload, minimizing the average of earliness and tardiness and so on.

Compared to exact methods, approximate methods, especially meta-heuristics, are better for solving FJSSP because meta-heuristics can obtain satisfactory solutions in a reasonable time. Meta-heuristics are more suitable for large-scale FJSSP. A music-inspired algorithm, discrete harmony search (DHS), and a nature-inspired algorithm, artificial bee colony (ABC), are investigated and improved to solve FJSSP with constraints.

Two encoding and decoding methods are developed for solution representation. Several simple and ensemble heuristics are employed to initialize population. An effective heuristic, named MinEnd heuristic is proposed to generate a high quality initial solution. Dynamic grouping strategies of harmony memory and new crossover method for improvising new harmonies are proposed. Effective local search operators are proposed to improve algorithms' performance. For the rescheduling operator, three re-scheduling strategies are proposed and compared. A two-stage discrete harmony search 
algorithm and a two-stage artificial bee colony algorithm are proposed for rescheduling FJSSP with remanufacturing constraints.

Extensive experiments have been conducted to test the performance of the proposed algorithms for solving FJSSP with remanufacturing constraints. The instance sets used in this thesis include three sets of benchmark instances and two sets from remanufacturing industry. Single objective, multiobjective and Pareto-based multi-objective formulations are considered in this thesis. The proposed algorithms are compared to more than ten existing heuristics and algorithms. The comparisons with literature show the effectiveness and efficiency of the proposed heuristics and algorithms. 


\section{List of Figure}

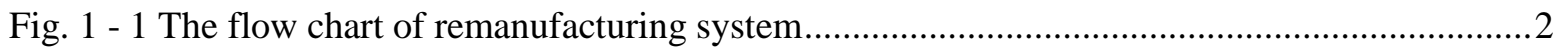

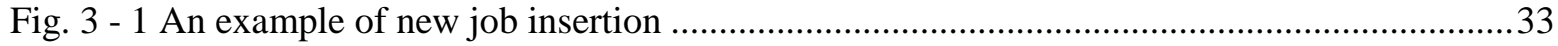

Fig. 3 - 2 An example of increasing processing time ....................................................................... 34

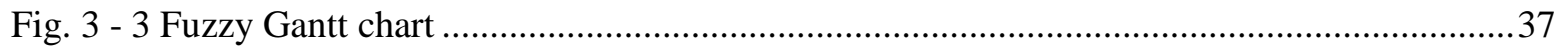

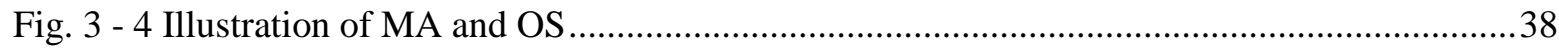

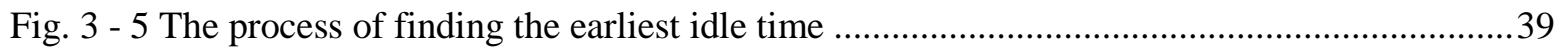

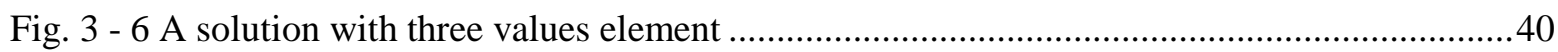

Fig. 3 - 7 Fuzzy Gantt chart of the three values element encoding and decoding ............................41

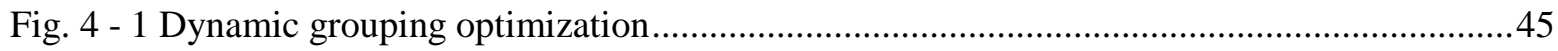

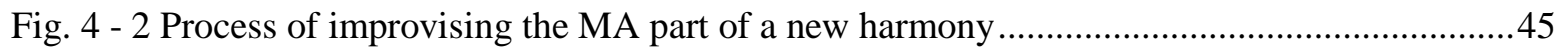

Fig. 4 - 3 Illustration of the crossover operator for OS part of a new harmony ...............................46

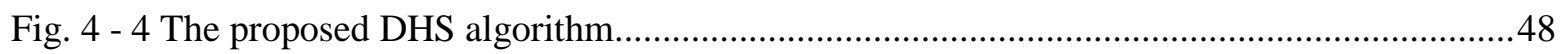

Fig. 4 - 5 Gantt chart of instance MK01 with makespan 40 ........................................................50

Fig. 4 - 6 Gantt chart of the solution $(24,3)$ for Kacem instance $8 \times 8$............................................54

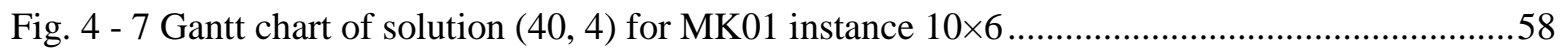

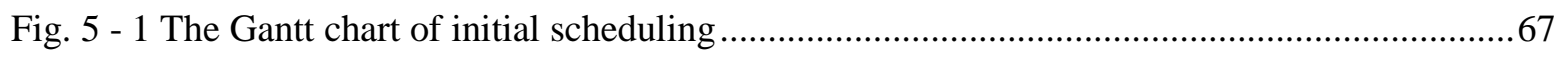

Fig. 5 - 2 The Gantt chart by re-scheduling strategy I ...............................................................67

Fig. 5 - 3 The Gantt chart by re-scheduling strategy II.............................................................6

Fig. 5 - 4 The Gantt chart by re-scheduling strategy III.................................................................6

Fig. 5 - 5 Scheduling and re-scheduling results for instance ….....................................................71

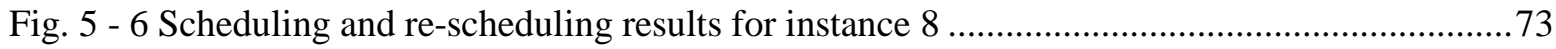

Fig. 6 - 1 An example Gantt chart for increasing processing time ….............................................75

Fig. 6 - 2 An example Gantt chart for new job insertion ............................................................. 75

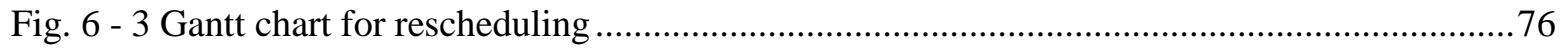

Fig. 6 - 4 The value of makespan with iteration ........................................................................ 81

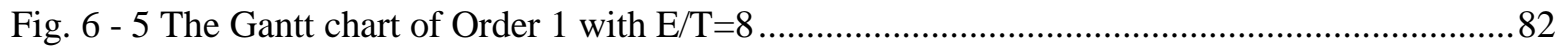

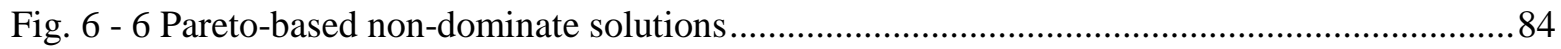

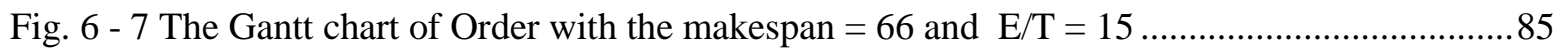




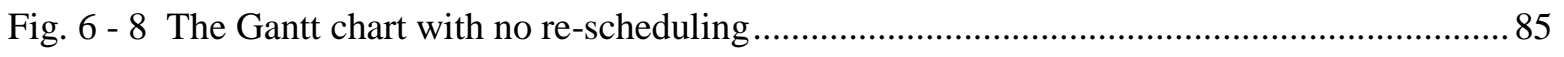

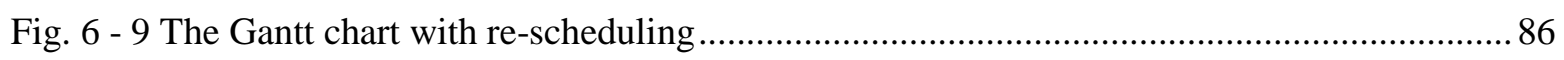

Fig. 6 - 10 The Gantt chart after inserting job Shaft sleeve 3 ..................................................... 86

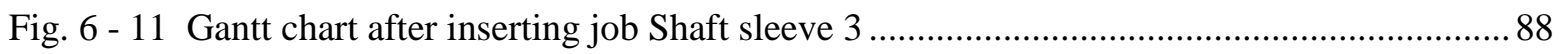

Fig. 6 - 12 Gantt chart after rescheduling for job Shaft sleeve 3 ................................................ 88

Fig. 7 - 1 Fuzzy Gantt chart by proposed heuristic rule.............................................................. 93

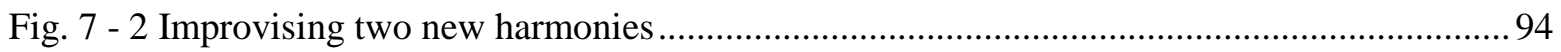

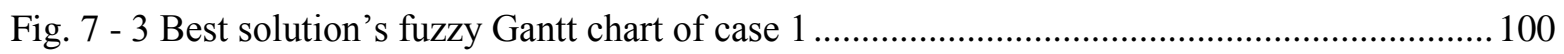

Fig. 7 - 4 Convergence curve of FDHS for eight instances in set two........................................... 101

Fig. 7 - 5 Best solution's fuzzy Gantt chart of instance Reman 2 ................................................. 102

Fig. 8 - 1 Best maximum fuzzy completion time Gantt chart of case 1 by FABC .......................... 115

Fig. 8 - 2 Best maximum fuzzy completion time Gantt chart of Reman 2 by FABC ...................... 119

Fig. 8 - 3 Maximum fuzzy completion time convergence curves of case 5 and Reman 8 ............. 120

Fig. 8 - 4 Best maximum fuzzy machine workload Gantt chart of case 1 by FABC....................... 123

Fig. 8 - 5 Best maximum fuzzy machine workload Gantt chart of Reman 2 by FABC .................. 124

Fig. 8 - 6 Maximum fuzzy machine workload convergence curves of case 5 and Reman 8 ........... 125

Fig. 9 - 1 An example Gantt chart for FJSSP with fuzzy processing time .................................... 127

Fig. 9 - 2 The Gantt chart by rescheduling strategy II ................................................................ 128

Fig. 9 - 3 The Gantt chart after rescheduling new job only ...................................................... 128

Fig. 9 - 4 Gantt chart of instance Reman 2 with minimum maximum fuzzy completion time......... 138

Fig. 9 - 5 First rescheduling Gantt chart with best maximum fuzzy completion time ...................... 139

Fig. 9 - 6 Second rescheduling Gantt chart with best maximum fuzzy completion time ................. 140

Fig. 9 - 7 Gantt chart of instance Reman 2 with minimum machine workload ................................ 148

Fig. 9 - 8 First rescheduling Gantt chart with best fuzzy machine workload ................................. 149

Fig. 9 - 9 Second rescheduling Gantt chart with best fuzzy machine workload .............................. 150 


\section{List of Table}

Table 3 - 1 An example of the model based on the most probable processing time ..........................34

Table 3 - 2 An example of triangle fuzzy number for modeling uncertain processing time...............36

Table 3 - 3 An example of fuzzy processing time for encoding and decoding ................................40

Table 4 - 1 The makespan results of BRdata by five algorithms ................................................50

Table 4 - 2 Pareto-based multi-objective results of five Kacem instances ......................................52

Table 4 - 3 Number of Non-dominate solutions and quality metric (QM) for 5 Kacem instances....53

Table 4 - 4 Diversification metric (DM) for 5 Kacem instances .....................................................54

Table 4 - 5 Pareto-based multi-objective results for BRdata .......................................................56

Table 4 - 6 Number Non-dominate solutions (NNdS) and quality metric (QM) for BRdata .............57

Table 4 - 7 Diversification metric (DM) for 10 BRdata instances...................................................57

Table 5 - 1 The data of eight remanufacturing instances ..........................................................6

Table 5 - 2 Scheduling and re-scheduling results for small-scale instances ...................................65

Table 5 - 3 Scheduling and re-scheduling results for large-scale instances....................................66

Table 5 - 4 Machine release time, job release time and corresponding operations...........................66

Table 5 - 5 Results of two benchmark sets by all compared algorithms .........................................69

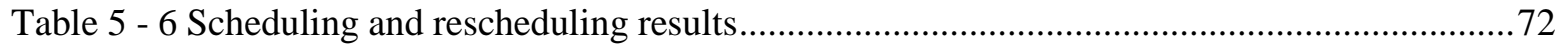

Table 6 - 1 The range of processing time of all jobs in Order 1 ................................................... 78

Table 6 - 2 The range of processing time of all jobs in Order 2 ................................................. 78

Table 6 - 3 The results of Makespan criteria by three algorithms .................................................8 80

Table 6 - 4 The complete times of all jobs with best makespan in two Orders ................................8 81

Table 6 - 5 The results of E/T criteria by three algorithms ............................................................ 82

Table 6 - 6 The completion times of all jobs with best E/T in Order 2 .........................................82

Table 6 - 7 Pareto-based non-dominated solutions by three algorithms ..........................................83

Table 6 - 8 The operators and corresponding range of processing times of Shaft 2 ........................87

Table 7 - 1 An example of FJSSP fuzzy processing time ..........................................................92

Table 7 - 2 Maximum fuzzy completion time results by six heuristic rules .....................................96

Table 7 - 3 Seven compared algorithms' results for five cases in set one .......................................97 
Table 7 - 4 Seven algorithms' CPU time (s) for five cases in set one ...........................................98

Table 7 - 5 FDHS and MinEnd results for eight instances in set two ...........................................99

Table 7 - 6 Average CPU time and generation of FDHS for eight instances in set two...................99

Table 8 - 1 Maximum fuzzy completion time of five cases by six heuristics................................. 109

Table 8 - 2 Maximum fuzzy machine workload of five cases by six heuristics ............................... 110

Table 8 - 3 Maximum fuzzy completion time by eight algorithms for five cases ............................ 112

Table 8 - 4 CPU time of eight algorithms for five cases .......................................................... 113

Table 8 - 5 Maximum fuzzy completion time for eight Reman instances ...................................... 115

Table 8 - 6 CPU time and generation for maximum fuzzy completion time .................................. 117

Table 8 - 7 Maximum fuzzy machine workload by EABC and improvement to six heuristics ....... 120

Table 8 - 8 Maximum fuzzy machine workload results for eight Reman instances ......................... 121

Table 8 - 9 Average CPU time and generation for maximum fuzzy machine workload objective .. 122

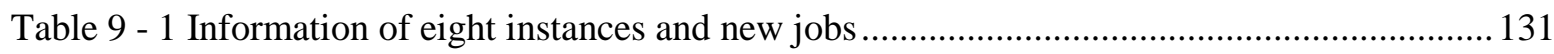

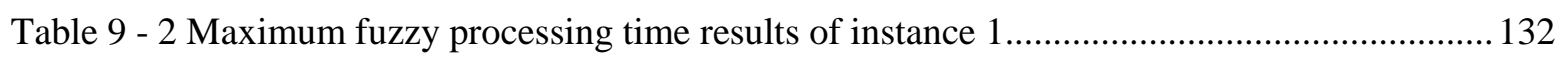

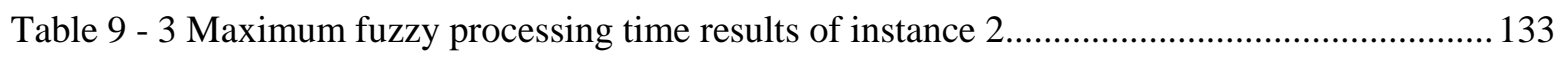

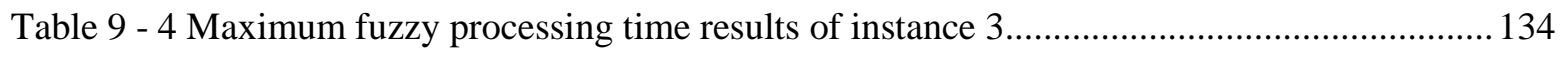

Table 9 - 5 Maximum fuzzy processing time results of instance 4 ............................................... 135

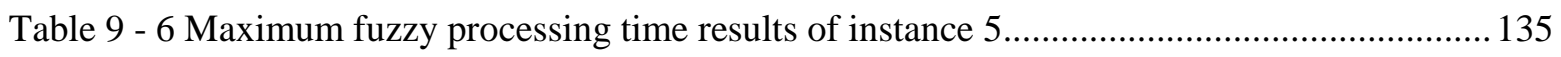

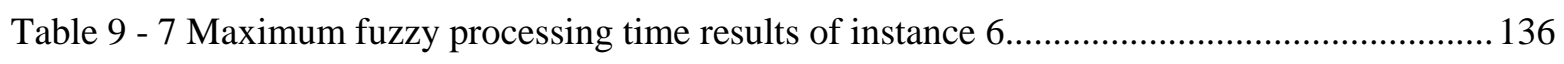

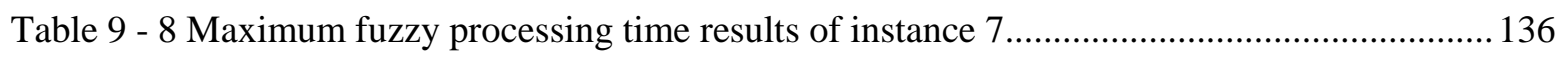

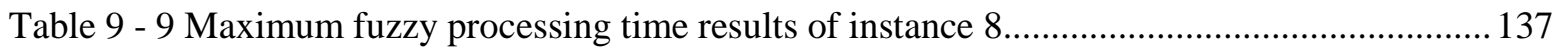

Table 9 - 10 Maximum fuzzy machine workload results of instance 1 ........................................ 142

Table 9 - 11 Maximum fuzzy machine workload results of instance 2 ....................................... 143

Table 9 - 12 Maximum fuzzy machine workload results of instance 3 ......................................... 143

Table 9 - 13 Maximum fuzzy machine workload results of instance 4 ......................................... 144

Table 9 - 14 Maximum fuzzy machine workload results of instance 5 ......................................... 144

Table 9 - 15 Maximum fuzzy machine workload results of instance 6 ........................................ 145

Table 9 - 16 Maximum fuzzy machine workload results of instance 7 ....................................... 146

Table 9 - 17 Maximum fuzzy machine workload results of instance 8 .......................................... 146

Table 9 - 18 TFABC algorithm's average CPU times for all instances and new job insertions....... 147 


\section{List of Abbreviations}

\begin{tabular}{ll} 
JSP & job shop problem \\
JSSP & job shop scheduling problem \\
FJSSP & flexible job shop scheduling problem \\
FIFO & first in first out \\
SPT & short processing time \\
EDD & earliest due date first \\
CDR & composite dispatch rules \\
GA & genetic algorithm \\
ACO & ant colony optimization \\
PSO & particle swarm optimization \\
DE & differential evaluation \\
HS & harmony search \\
DHS & discrete harmony search \\
PGDHS & Pareto-based grouping discrete harmony search \\
ABC & artificial bee colony \\
SA & simulated annealing \\
TS & tabu search \\
VNS & variable neighborhood search \\
HM & harmony memory \\
HMS & harmony memory size \\
HMCR & harmony memory consideration rate \\
PAR & pitch adjustment rate \\
MA & machine assignment vector \\
OS & operation sequence vector \\
TFN & triangle fuzzy number \\
QM & Duality metric \\
DM & diversication metric \\
\hline
\end{tabular}




\section{List of Symbols}

$\begin{array}{ll}J & \text { set of jobs } \\ M & \text { set of machines } \\ O_{i, j} & \text { operation } j \text { of job } i \\ c_{i, j} & \text { the completion time of operation } O_{i, j} \\ c_{i} & \text { the completion time of job } J_{i} \\ C_{M} & \text { the maximum completion time, Makespan } \\ E / T & \text { the mean of earliness and tardiness } \\ R P I & \text { the relative percentage increase } \\ C_{W} & \text { the maximum machine workload } \\ A R P I & \text { the average relative percentage increase } \\ n & \text { number of job } \\ m & \text { number of machine } \\ \text { Di } & \text { the due date of job } J_{i} \\ X_{i} & i^{\text {th }} \text { harmony vector in the HM } \\ X_{n e w} & \text { new harmony }\end{array}$





\section{Chapter 1}

\section{Introduction}

Remanufacturing is the process of disassembly and recovery at the module level and, eventually, at the component level. It requires the repair or replacement of worn out or obsolete components and modules. Parts subject to degradation affecting the performance or the expected life of the whole are replaced. Remanufacturing is a form of a product recovery process that differs from other recovery processes in its completeness: a remanufactured machine should match the same customer expectation as new machines. Lund [1] was the first one to define and discuss the remanufacturing problem. Krupp [2] perfected the concept of remanufacturing and predicted the future of remanufacturing engineering. There are seven characteristics of remanufacturing as follows [3]:

1. Routing uncertainty and processing time uncertainty. The condition of recovered parts is unknown until inspected. This leads to stochastic routing and lead times.

2. Balancing returns with demand. There is a problem of imperfect correlation between the supply of cores and the demand for remanufacturing units.

3. The disassembly of returned products. The disassembly and subsequent release of parts to the remanufacturing operations requires a high degree of coordination with reassembly to avoid high inventory levels or poor customer service. 
4. Uncertainty in materials recovered. Some parts that are not recoverable must be replaced by new parts. This characteristic complicates resource planning and scheduling since the volumes of parts recovered and required processing will be a stochastic variable. Remanufacturing production schedule is to be tightly integrated with dependent demand inventory.

5. The requirement for reverse logistics network. It is the determination of how products will be collected and transported to the operation, involving a set of parallel decisions, such as the number of collection centers, method and frequency of transport.

6. Materials/parts matching requirements. Units are often composed of serial number specific parts and components, along with common ones. Coordination between disassembly and reassembly is critical if customer due dates are to be met.

7. Uncertainty in the timing and the quality of returns. The number of products returned and the timing are factors that cannot be controlled by remanufacturers, due to factors, such as stage of product lifecycle and the rate of technological change.

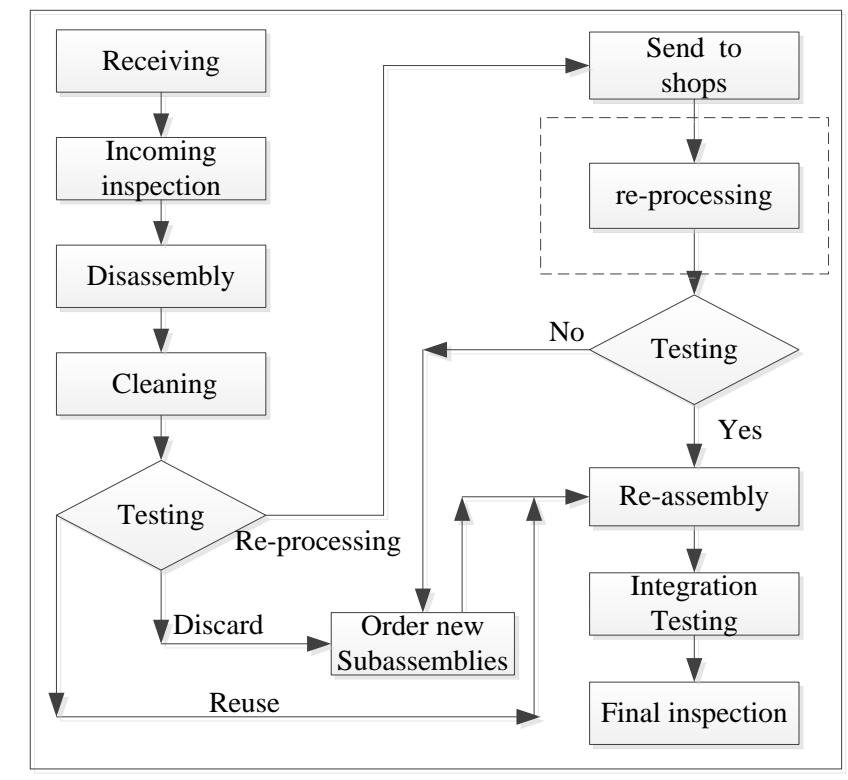

Fig. 1 - 1 The flow chart of remanufacturing system

Fig. 1 - 1 shows the process of remanufacturing, which is developed from the remanufacturing model in literature [4]. For remanufacturing, each return production has a set of subassemblies. After cleaning and testing each subassembly, the subassemblies can be classified into one of the three groups: (1) to be reused, (2) to be discarded and order new one or (3) to be re-processed. The subassembly that can be processed will be sent to shop floor for re-processing. After testing, the re-processed subassemblies will be re-assembled for final inspection. 
In recent years, many researchers focused on remanufacturing theory research and industry application. Guide $\mathrm{Jr}[5]$ reported the production planning and control in remanufacturing for industry practice and research needs. Guide Jr [6] analyzed the capacity planning in remanufacturing environment. Guide [7] examined the impact of product structure complexity on other managerial and operational decisions in a remanufacturing environment. Depuy [4] proposed a methodology for production planning within facilities involved in the remanufacturing of products. Teunter [8] developed a polynomial-time dynamic programming heuristic for the minimization of cost in manufacturing and remanufacturing engineering. Grubbstrom [9] proposed a model to analyze how labor costs, material costs and budget influence the optimal production decisions in remanufacturing engineering. Guide $\mathrm{Jr}$ [10] considered the production planning when inputs have different and uncertain quality levels and discussed different decision variables in remanufacturing engineering. M.L. Junior [11] reviewed the literature on production planning and control in remanufacturing. Seventy-six papers were examined and classified in the literature. However, there are only a handful of papers on reprocessing scheduling in remanufacturing.

The literature on Production Planning \& Scheduling in remanufacturing is dominated by quantitative approaches. The majority of these quantitative models developed are generic; namely, not associated with any kind of specific remanufacturing operation. The presence of complicating characteristics does not necessarily mean it is an area that has been fully addressed. In fact, because most studies are theoretical, the need for more practical research on each PPS activity based on the complicating characteristics is evident.

The re-processing of returns in remanufacturing can be modelled as FJSSP with constraints. The characteristics 1) and 7) can be modelled as constraints for scheduling re-processing. Hence, the reprocessing scheduling problem in remanufacturing can be solved as FJSSP scheduling problem with two constraints. One constraint is that processing time may become larger or smaller in re-processing shop floor for characteristic 1). Another constraint is new job coming and inserting into scheduled sequences for characteristic 7).

\subsection{Flexible job shop scheduling problem}

Job shop scheduling problem (JSSP) is to solve $n$ jobs processed on $m$ machines[12]. Each job comprises multiple operations while each machine can process multiple operations. Each machine can process only one operation at a time and each operation can be processed on only one machine at a time. The purpose of scheduling is to assign all operations on machines to achieve best scheduling 
objectives. In JSSP, each job has a predetermined routing. The operations of the same job have precedence constraints and are processed in a sequential order.

The flexible job-shop scheduling problem (FJSSP) is an extension of classical JSSP with practical applications[13]. FJSSP is a combination of the job shop and the parallel machine environments. Instead of $m$ machines in series, there are multiple work centers with each work center consisting of a number of identical machines in parallel. Each job has its own route to follow through the shop and requires processing at each work center on only one machine and any machine in the work center can process the job. FJSSP includes two sub-problems: 1. machine assignment to select a machine from a set of candidate machines for each operation; 2. operation sequencing to schedule the operations on all machines to obtain a feasible solution. In classical JSSP problem, one operation can be processed on only one machine. Hence, the FJSSP is more difficult than classical JSSP. There is a great variety of real-world problems that can be modeled as FJSSP, e.g., optimization of crane operations, simulation and optimization of transport systems, and scheduling in real manufacturing and remanufacturing systems.

\section{Features of FJSSP}

FJSSP has the following features:

\section{- Computational Complexity}

Comparing to classic JSSP problem, FJSSP has an additional step of selecting a processing machine from candidate machine set for each operation. Hence, FJSSP has higher computation complexity than classic JSSP problem. FJSSP has been proven as an NP-hard problem [14].

Discreteness

FJSSP is a typical discrete event problem. Operations are processed on a different instant of time and different machines. In FJSSP, a job arrival, a change in orders, an addition of machine and a machine breakdown are all discrete events. Hence, discrete event simulation, queuing theory are employed for FJSSP.

- Multi-objectives [15]

In different enterprise and different production environments, the objectives of FJSSP are different and varied. For completion time related criteria, FJSSP scheduling objectives include minimizing maximum completion time (Makespan), minimizing total completion time or average completion time and so on. For machine load related criteria, there are minimizing maximum machine load, minimizing total machine load or average machine load objectives. The due date related criteria are minimizing earliness or tardiness, minimizing average earliness or tardiness, minimizing total earliness and tardiness. There are also some other scheduling objectives in FJSSP, for example, cost related objectives. 
- Multi-constraints

Generally, the routing of each job is known before processing and the operations of a job have strict precedence. At the same time, the candidate machines are also known and the operations must be processed on one machine based on the precedence. In addition, there are many other constraints in different industries and different production environments, for example, uncertain processing time, new job insertion, maintenance activity, limited resources, overlapping operations, sequencedependent setup and transportation times and so on.

\section{Categories of FJSSP}

Based on the number of candidate machines, FJSSP can be divided into two categories: Total FJSSP and Partial FJSSP [16]. In Total FJSSP, each operation can be processed on any machine. In Partial FJSSP, some of the operations can be processed on all machines while the rest of operations can be processed on some of the machines from the machine set. In general, partial FJSSP is more applicable for the real production environment, as some machine cannot be used to process some of the operations.

\section{Research approach on FJSSP}

There are exact and approximate methods for solving FJSSP. Exact methods guarantee global optimal solutions. However, exact methods do not scale up with problem size, and hence are only suitable for small size problems and tend to be very slow. Approximate methods can obtain feasible solutions quickly but cannot guarantee global optimal solutions. Approximate methods are therefore very suitable for large size problems. They can find satisfactory solutions and solve real industry problems in limited time.

- Exact methods

Mathematical programming is the most used exact method for job shop scheduling problems, for example, Lagrangian relaxation and Decomposition method[17]. Most exact methods are based on a disjunctive graph. Branch-and-bound is also a commonly used method for FJSSP in early research [18]. Branch-and-bound consists of a systematic enumeration of all candidate solutions, where large subsets of fruitless candidates are discarded masse, by using upper and lower estimated bound of the problem being optimized.

- Approximate methods

Many approximate methods are proposed and employed for solving FJSSP in the past two decades. Priority dispatch rule is the earliest approximate method [19]. This method is based on the predetermined operation priority. Priority dispatch rule is simple and easy to realize. First in first out (FIFO) rule, shortest processing time (SPT) first rule and earliest due date first (EDD) rule are the usually employed priority dispatch rules. Some researchers have investigated composite dispatch rules 
(CDR) [20]. These CDR are the heuristic combination of single dispatching rules that aim to inherit the advantages of them.

Many meta-heuristics are employed and developed for FJSSP in recent years, for example, Genetic algorithm (GA) [21], ant colony optimization (ACO) [22], particle swarm optimization (PSO) [23], differential evolution (DE) [24], harmony search algorithm (HS) [25], artificial bee colony (ABC) [26], Simulated annealing (SA) and Tabu search (TS) [27] etc. The advantage of meta-heuristic is to obtain multiple approximate solutions quickly.

Local search is also an effective approximate method. Local search is usually used to find local best solutions. Variable local search (VNS) [28] is employed by many researchers to improve the convergence of algorithm for solving FJSSP.

\subsection{Motivation and purpose}

FJSSP is very important in both academic and application fields. Many researchers focus on FJSSP due to its wide applicability and inherent difficulty. However, most existing literature focuses on the theoretical research, benchmark instance and performance of different methods and algorithms. Few pay attention to the practical environment, special fields and constraints. Hence, how to solve the FJSSP in the practical environment and industry constrains is a challenge to researchers and engineers. This study is partly supported by Remanufacturing Scheduling System of A*Star (Agency for science, Technology and Research, Singapore) under Grant 1122904021.

This thesis modeled constrained FJSSP for remanufacturing industry. Two of the seven characteristics of remanufacturing are considered. The two characteristics are new job insertion, and the uncertain processing time of job operations. These two constraints are related to shop scheduling in remanufacturing environment. Two competitive meta-heuristics, discrete harmony search (DHS) and artificial bee colony (ABC) algorithms, are employed and developed for solving the FJSSP with remanufacturing constraints. The objectives are to minimize the completion time related criteria, machine workload related criteria and due date related criteria. The objectives of this thesis are to improve the production effectiveness of re-processing in remanufacturing industry and to reduce the material and production inventory cost. It can also make the enterprise_improve response times and satisfying customer requirements better.

\subsection{Original contributions}

The major contributions throughout the whole $\mathrm{PhD}$ thesis research period are summarized as follows: 
- We model the scheduling problem in remanufacturing industry using constrained FJSSP. Two scheduling related constraints, new job insertion and uncertain processing time, are considered as constraints. Rescheduling strategies are proposed for dealing with new job insertion constraints. The most probable processing time and triangle fuzzy number are employed to present the uncertain processing time.

- A discrete harmony search (DHS) algorithm is proposed to solve single objective, multi-objective and Pareto-based multi-objective FJSSP. We are the first to apply DHS algorithm on practical FJSSP with remanufacturing constraints. For FJSSP with two remanufacturing constraints, a twostage DHS algorithm is proposed to execute scheduling and rescheduling operators. A fuzzy DHS is proposed to solve FJSSP with uncertain processing time. These DHS algorithms are employed to solve benchmark instances and real instances from remanufacturing industry. Many existing algorithms are compared to demonstrate improved performance of proposed DHS algorithms.

- An artificial bee colony (ABC) algorithm is proposed to solve FJSSP with new job insertion for minimizing makespan objective. We are the first to apply ABC algorithm on practical FJSSP with remanufacturing constraints. For fuzzy processing time constraint, a fuzzy $\mathrm{ABC}$ algorithm is proposed to minimize fuzzy maximum completion time and fuzzy maximum machine workload. A two-stage fuzzy $\mathrm{ABC}$ algorithm is proposed to solve FJSSP with uncertain processing time and new job insertion constraints. These $\mathrm{ABC}$ algorithms are compared to many existing algorithms to demonstrate improved performance.

- To improve the quality of initializing solutions, many simple heuristics are employed to initialize population. An effective heuristics, named MinEnd heuristic, is proposed to initialize fuzzy algorithms' population. To test performance, MinEnd heuristic is compared to several simple heuristics. Multiple ensemble heuristics are proposed to solve the FJSSP and compared to proposed DHS and ABC algorithms.

For the feature of FJSSP, two encoding and decoding methods are proposed to represent the solution. Dynamic grouping harmony memory and new crossover method are proposed to improve DHS algorithms performance. An ensemble local search method is proposed to improve the speed of convergence in proposed DHS and $\mathrm{ABC}$ algorithms. The extensive experimental comparisons show the effective and efficiency of these operators over the state-of-the-art.

\subsection{Organization of the thesis}

This thesis is structured into ten chapters, beginning with this introduction. 
Chapter 2 presents harmony search algorithm and artificial bee colony algorithm in detail. Based on research approaches and scheduling objectives, we review the literature on solving FJSSP.

Chapter 3 models FJSSP. Two characteristics of remanufacturing industry, new job insertion and uncertain processing time, are modeled as two constraints of FJSSP. Two encoding and decoding methods are proposed to make the FJSSP solution suitable for DHS and ABC algorithms.

Chapter 4 proposes a dynamic group discrete harmony search algorithm for single objective and Pareto-based multi-objective FJSSP. Simple heuristics are employed for initializing population. Harmony memory dynamic grouping and local search methods are proposed to improve algorithm performance. To demonstrate improved performance, the experiments results for 15 benchmark instances are compared to existing algorithms.

Chapter 5 proposes a two-stage artificial bee colony algorithm (TABC) for scheduling FJSSP with new job insertion. An ensemble local search is proposed to improve the convergence of TABC. Three re-scheduling strategies are proposed for new job insertion. The three re-scheduling strategies are compared and discussed. To demonstrate improved performance, TABC are compared to several simple heuristics, hybrid heuristics and DHS algorithm proposed in Chapter 4.

Chapter 6 proposes a two-stage discrete harmony search (TDHS) algorithm for scheduling FJSSP with new job insertion and processing time increasing. Rescheduling strategy is executed when the processing time increases or the new job is inserted into existing scheduling solution. A local search algorithm is employed to speed up the convergence of the proposed TDHS algorithm. Computational results show that the real remanufacturing problems are solved effectively and efficiently. Scheduling and rescheduling results are satisfactory and can be used in practice.

Chapter 7 proposes a fuzzy DHS (FDHS) algorithm for single objective FJSSP with fuzzy processing time. Triangle fuzzy number (TFN) is used to show the uncertainty of processing time. A heuristics, named MinEnd, is proposed to initialize population. Three fuzzy number operators are employed to compare and rank triangle fuzzy number. The proposed FDHS algorithm is compared to six metaheuristics for five benchmark instances. Eight instances from remanufacturing industry are solved by FDHS algorithm.

Chapter 8 proposes a fuzzy ABC (FABC) algorithm for multi-objective FJSSP with fuzzy processing time. The objectives are to minimize the maximum fuzzy completion time and the maximum fuzzy machine workload. The MinEnd heuristic in Chapter 8 is employed to initialize population. To test performance, FABC algorithm is compared to six meta-heuristics and FDHS algorithm for maximum fuzzy completion time criterion. For maximum fuzzy machine workload, FABC algorithm is compared to six heuristics. 
Chapter 9 proposes a two-stage fuzzy ABC (TFABC) algorithm for FJSSP with fuzzy processing time and new job insertion constraints. Eight instances and nineteen insertions are solved by two-stage fuzzy ABC algorithm. The results and comparisons show the effectiveness and efficiency of TFABC algorithm for solving multi-objective FJSSP with multiple constraints.

Chapter 10 presents the conclusions and future work. 


\section{Chapter 2}

\section{Literature Review}

This Chapter reviews the literature for solving the flexible job shop scheduling problem. Harmony search algorithm and artificial bee colony algorithm are introduced and reviewed in detail.

\subsection{Research approach}

There are exact and approximate methods for solving FJSSP. Mathematical Programming and Branch and bound methods are two major exact methods for shop scheduling problems. There are many different mathematical programming methods, e.g. linear programming, dynamic programming, mixed-integer linear programming and so on. The principle of mathematical programming is to describe the scheduling problem as a mathematical programming model. The problem model is used to obtain the scheduling solutions.

Branch and bound (BB or $\mathrm{B} \& \mathrm{~B})$ is an algorithm design paradigm for discrete and combinatorial optimization problems, such as the job shop scheduling problems. Branch-and-bound consists of a systematic enumeration of candidate solutions by means of state space search: the set of candidate solutions is thought of as forming a rooted tree with the full set at the root. Branch-and-bound explores branches of the tree, which represent subsets of the solution set. Before enumerating the 
candidate solutions of a branch, the branch is checked against upper and lower estimated bounds on the optimal solution, and is discarded if it cannot produce a better solution than the best one found so far by the algorithm.

The advantages of exact methods include that they can be employed to analyze most complex optimization problems in real-life, the exact methods can divide the solved problem to small subproblems and make the solving process easier, the exact method can guarantee the optimal solutions. The exact methods also have some disadvantages. The combinatorial optimization problems and some real-life constraints cannot be described in the programming model. The performance of branch-andbound is based on the efficient estimation of the lower and upper bounds of a region in the search space.

Exact methods do not scale up with problem size. The computational complexity of exact methods will rapidly growth with the scale of the problems. Exact methods are only suitable for small sized problems and cannot satisfy the real-life requirements for large scale problems.

Compared to exact methods, approximate methods can obtain feasible and acceptable quality solutions quickly. Approximate methods are therefore very suitable for large size problems. They can find satisfactory solutions and solve real industry problems in limited time.

This section mainly reviews approximate methods for solving FJSSP. Approximate methods consist of three classes: 1. Priority dispatch rule, 2. Heuristic and 3. Meta-heuristic. We review related literature based on these three classes, especially the meta-heuristics. For meta-heuristics, we review related literature based on the different algorithm types.

\section{Priority dispatch rule}

Priority dispatch rules are the earliest approximate method [18]. These rules are based on the predetermined operation priority. Priority dispatch rules are simple and easy to realize. First in first out (FIFO), shortest processing time first (SPT) and earliest due date first (EDD) rules are the usually employed priority dispatch rules. Some researchers have investigated composite dispatch rules (CDR) [19]. These CDR are the combination of single dispatching rules that aim to inherit the advantages of them.

\section{Heuristic}

Heuristics are simple and can obtain a feasible solution in very limited time. For FJSSP, there are some heuristics for machine assignment, some heuristics for operation sequences and some heuristics for both machine assignment and operation sequence.

1) Heuristics for machine assignment.

- Local minimum processing time heuristic [16] 
This heuristic considers the processing time and starts from the operation with the minimum processing. For each operation, the machine with minimum processing time is fixed and assigned.

- Global minimum processing time heuristic [26]

This rule considers both the processing time and the workload of machines and starts from the operation with the global minimum processing time. The processing time is added to the machine workload. For every operation, the machine with the minimum workload is fixed and assigned. The machine's workload is updated. This rule considers the global workload among all machines and can potentially find better makespan.

- Two-step greedy heuristic [29]

The operations are sorted in ascending order based on the number of selectable machines with nondecreasing order of processing times to break ties when there is an equal number of selectable machines. The machines are sorted in their workload non-decreasing order. One operation is taken from the operations list and the first machine that belongs to the machine list is assigned to the operation. The workload of this machine is updated and the machine ranking is also updated. The process iterates until all operations have been assigned to machines.

- Minimum completion time heuristic [123]

This rule is based on the local minimum processing time heuristic. Two machines are selected for one operation that has more than one candidate machines. One machine has the minimum processing time and another machine has the earliest start time. The completion time on these two machines are calculated. The machine with the smaller completion time is fixed and assigned.

2) Heuristics for operation sequences

- Most work remaining [26,30]

This method firstly orders the jobs in a descending order based on the remaining processing time. The job with the most remaining processing time will be selected first and put into the operation sequence. The iteration is repeated until all operations of all jobs are sequenced.

- Most operation remaining [26,30]

This heuristic selects jobs based on the number of remaining operations. The job with the most remaining operations unprocessed is selected first.

- Shortest processing time (SPT) [30]

In this approach, the operation with the shortest processing time is selected from the next executable operations.

3) Heuristic for both machine assignment and operation sequences 
- Newton-based hierarchical heuristic [31]

In this heuristic, an adaptation is made to consider discrete variables and also to redefine the stopping criterion. Each new solution improves simultaneously all the objectives till the stopping criterion is met.

- Greedy heuristic [32]

This heuristic considers jobs one after another according to a fixed job sequence. The operation sequence is fixed by applying the polynomial algorithm. The machine assignment is based on priority rule.

- Filtered-beam-search based heuristic $[33,34]$

Filtered beam search is an extension of beam search. The filtered beam search procedure uses both crude and accurate evaluations in two phases: filtering phase and beam selection phase. A successful filtered beam search based heuristic for FJSSP should solve four major elements:

$>$ Search tree representation to define a solution space.

$>$ Determination of beam width and filter width.

$>$ Branching scheme.

$>$ Local and global evaluation functions selection.

- Constructive procedure based heuristic $[35,36]$

This approach is motivated by the idea of developing a constructive heuristic that considers simultaneously many factors affecting the solution quality and intelligently balances their effects. This algorithm has a simple structure and great flexibility, is easy to implement, and requires very little computational time.

- Variant of dispatch heuristic [37]

The dispatch heuristic considered two variants: (i) an ordered variant, where the priority dispatching rules were applied in a predefined order, and (ii) a randomized variant, where the user could assign probabilities (weights) to the priority rules. Using the information of the number of units and due dates requested by the customer, the algorithm provided the sequence of operations that must be performed on each machine, as well as the start and completion times of operations. In order to reduce the impact of unexpected events on a generated schedule, several robust rules were considered. The obtained results substantially improved the former method used in the company in terms of minimizing average tardiness. Additionally, other important benefits were obtained, including significant saving in the time spent on scheduling, simplicity of use of the proposed procedure, robustness against unexpected events, reduction of idle times, improvement of decision-making information, and improvement of on-time delivery performance.

- Combination heuristic [38] 
In the combination heuristic, a simulation based on earliest due date (EDD), the operations' lowest level code (LLC) of the bill of materials and the longest processing time (LPT) is proposed. A reallife weapons production factory was used as a case study to evaluate the performance of the proposed algorithm. Due to the high penalty of delays in military orders, the on-time delivery rate was the most important performance measure and makespan was the next most important measure.

- Discrepancy search heuristic [39]

In the heuristic, a variant of the climbing discrepancy search approach was developed for solving FJSSP. Various neighborhood structures related to assignment and sequencing problems were employed to enhance the results quality.

- Mixed integer goal programming model [40]

Two mixed integer goal programming models are formulated for FJSSP separable/non-separable sequence dependent setup times. In the first model, the sequence dependent setup times were nonseparable. In the second one, they were separable. The second model was obtained from the first one with a minor modification. The formulation of the models was described on a small sized numerical example and the solutions were interpreted. Finally, computational results were obtained on test problems.

- Other heuristics

He W [41] proposed a multi-strategies based approach to schedule FJSSP with machine breakdown. In pre-scheduling, an idle time insertion strategy was put forward. In the policy, an idle time equal to repair time was inserted into an appropriate position of each machine according to the machine's breakdown nature. A route changing strategy combined with right-shift policy was proposed to keep the rescheduling as stable and robust as possible. In the policy, whether to right shift or route change dependent on the cost of archiving robustness and stability. Based on the two strategies, algorithms dealing with idle time insertion, right-shift scheduling, and route changing scheduling were designed. The computational results show the effectiveness of the strategies and algorithms compared with other strategies. Also to solve FJSSP with machine breakdown, Sun HD [42] proposed a game theory based approach. In the approach, pre-scheduling without considering machine failure was generated. The operations affected by machine failure were obtained according to time at which machine breakdown happened. The decision made by each side obtained better robust and stable performance. To achieve the optimal objects, Nash Equilibrium (NE) solution for each side was achieved. Considering NE solution might not exist or there were more than one NE solutions, concepts of ideal NE solution and near NE solution were proposed. To avoid huge decision searching space when the number of operations was more than two, an NE searching algorithm under multi-stage game based on the two concepts was also proposed to find NE or near NE solutions. 


\section{Meta-heuristics}

Many meta-heuristics are employed and developed to solve FJSSP in recent years. This section will mainly review the literature based on different meta-heuristics.

- Genetic algorithm (GA)

GA is a widely used algorithm to solve FJSSP. Chan FTS [43] developed a GA based approach to solve FJSSP with resource constraints. Using the approach, a resource-constrained operation machines assignment problem and flexible operation sequencing problem could be solved iteratively. In the connection, the flexibility embedded in the flexible shop floor, which was important to today's manufacturers, could be quantified under different levels of resource availability. Ho NB [44] proposed a GA based architecture (LEGA) for learning and evolving FJSSP. Unlike the canonical evolution algorithm, random elitist selection and mutational genetics were assumed through LEGA. The knowledge extracted from the previous generation was used to influence the diversity and quality of offsprings. In addition, the architecture specified a population generator module that generated the initial population of schedules and trained the schemata-learning module. Experimental results indicated that an instantiation of LEGA outperformed the compared approaches in computational time and quality of schedules. Gao J. [30] proposed a GA algorithm with a local search based on criteria path to solve FJSSP with maintenances.

Pezzella F [16] studied on a genetic algorithm with different initial strategies to solve FJSSP. The algorithm integrated different strategies for generating the initial population, selecting the individuals for reproduction and reproducing new individuals. De Giovanni L [45] proposed an improved GA to solve the distributed and flexible job shop scheduling problem. A greedy decoding procedure exploited the flexibility and determined the job routings. Besides traditional crossover and mutation operators, a new local search based operator was used to improve available solutions by refining the most promising individuals of each generation. Mati Y [46] proposed a GA for practical FJSSP with blocking constraints. The model and algorithm were used to a company to design a new production workshop. Numerical experiments using real data were presented and analyzed. The authors showed how these results were used to support choices in the design of the workshop.

Ai-Hinai N [47] addressed a hybrid GA to solve robust and stable FJSSP with random machine breakdown. The first stage optimized the primary objective, minimizing makespan in the literature, where all the data was considered deterministic with no expected disruptions. The second stage optimized the bi-objective function and integrated machines assignments and operations sequencing with the expected machine breakdown in the decoding space. An experimental study and analysis of variance was conducted to study the effect of different proposed measures on the performance of the 
obtained results. Results indicated that different measures had different significant effects on the relative performance of the proposed method. Chen James C. [48] proposed a GA and grouping GA for FJSSP with parallel machines. Gholami M. [49] researched on an integrating simulation GA for scheduling dynamic FJSSP. To solve multi-objective FJSSP, Rahmati SHA [50] studied a multiobjective genetic algorithm. In the study, non-dominated ranking are adapted. Chiang TC [51] addressed a simple and effective GA for scheduling FJSSP which utilized effective genetic operators and maintains population diversity carefully. A main feature of the algorithm was its simplicity. It needs only two parameters.

For FJSSP with overlapping in operations, Demir Y [52] presented an effective two-steps GA. Zhang GH [53] proposed a GA with high quality initial population to solve FJSSP. Global selection and local selection were designed to improve the quality of the initial population. Wang XJ [54] presented a multi-objective genetic algorithm (MOGA) for FJSSP. The algorithm was based on immune and entropy principle. MOGA applied Pareto-optimality to fitness scheme. To solve FJSSP with machine disruption, Wang YM [21] created a novel genetic algorithm. In the algorithm, a very special chromosome encoding was designed to adapt to disruption. The algorithm was compared to a rightshifting re-scheduler and a pre-scheduler algorithm. For FJSSP with fuzzy processing time, Lei DM [55] proposed a co-evolutionary genetic algorithm (CGA). A new crossover operator and a modified tournament selection are proposed in the study. To solve FJSSP with sequence dependent setups, Defersha FM [56] presented a parallel genetic algorithm. The algorithm runs on a parallel computing platform. Numerical examples showed that parallel computing could greatly improve the computational performance of the algorithm.

- Ant colony optimization (ACO)

Many researchers employed and developed ACO to solve FJSSP. Rossi A [57] proposed an ACO algorithm for FJSSP with routing flexibility and separable setup times. The optimization problem for a real environment, including parallel machines and operation lag times, was approached by means of an effective pheromone trail coding and tailored ant colony operators for improving solution quality. The method used to tune the system parameters was also described. The algorithm was tested by using standard benchmark problems, properly designed for a typical FMS layout. The effectiveness of the proposed system was verified in comparison with alternative approaches. Xing LN [22] proposed a knowledge-based ACO (KBACO) algorithm for FJSSP. The algorithm integrated ACO and a knowledge model. The knowledge-based system learned available knowledge from ACO and applied the existing knowledge to guide the searching. The authors also designed and evaluated an ACO based simulation model to solve multi-objective FJSSP. Yao BZ [58] presented an improved ACO algorithm for FJSSP. Three improved strategies, transition rule with adaptive parameters, crossover operation 
and pheromone updating strategy with adaptive parameters were introduced to enhance the performance of ACO. For multi-objective FJSSP, Luo DL [59] proposed an ACO algorithm which combined SPT rule and local search method to improve performance. Rossi A [60] researched an ACO algorithm with reinforced pheromone relationships to solve FJSSP with sequence-dependent setup and transportation time. Huang RH [61] developed a two pheromone ACO (2PH-ACO) to solve FJSSP. Computational results indicated that $2 \mathrm{PH}-\mathrm{ACO}$ performed better than $\mathrm{ACO}$ in terms of sum of earliness and tardiness time.

- Particle swarm optimization (PSO)

Boukef H [62] proposed an algorithm inspired from PSO for FJSSP with makespan criterion. The experiments and discussions showed that the algorithm was better than the compared GA algorithm. Liu HB [63] used a multi-swarm PSO algorithm to solve multi-objective FJSSP. The authors theoretically proved that the multi-swarm synergetic optimization algorithm converged with a probability of one towards the global optima. To solve Pareto-based multi-objective FJSSP, Moslehi G. [64] proposed a particle swarm optimization with local search. The results indicated that the algorithm satisfactorily captured the multi-objective flexible job-shop problem and competed well with the compared approaches. Nourali S [65] employed a PSO based algorithm for flexible assembly job shop scheduling problem with sequence dependent setup times. The literature focused on real industry application. Practice industry instances were solved to demonstrate the effectiveness of the algorithm.

- Differential evolution (DE)

To solve FJSSP, Yuan Y [66] proposed a hybrid differential evolution (HDE). In the algorithm, a conversion mechanism was developed to make the DE that worked on the continuous domain adaptive to explore the discrete problem space of the FJSSP. A local search algorithm based on the critical path was embedded in the HDE framework to balance the exploration and exploitation by enhancing the local searching ability. In addition, a speed-up method to find an acceptable schedule within the neighborhood structure was presented to improve the efficiency of whole algorithms. In addition, Xu XL [67] proposed a hybrid discrete differential evolution (HDDE) algorithm for lot splitting with capacity constraints in flexible job scheduling. The HDDE algorithm also employed criteria path based local search to improve performance. The simulation results showed that the HDDE algorithm could effectively solve the practical lot splitting problems with equipment capacity constraints.

- $\quad$ Simulated annealing (SA)

A few papers use just SA to solve FJSSP. Fattahi P [68] proposed a SA based hierarchical approach to solve large-scale FJSSP instances. To evaluate the validity of the proposed SA algorithm, the results 
were compared with the optimal solution obtained by the Branch and Bound method. The authors also prove that the SA based algorithm could be applied easily to real factory conditions. SA is used in many hybrid algorithms for FJSSP. The literature for these hybrid algorithms will be reviewed in hybrid algorithms.

- Tabu search (TS)

TS is also a widely used meta-algorithm for FJSSP. Logendran R [69] proposed six TS-based metaheuristics (TSH1-TSH6). The results showed that, as the problem size increases, TSH3 with fixed tabu-list size and long-term memory was preferred over the other heuristics. Further, the branchand-bound technique, by failing to identify as goad a solution as that determined by the heuristics (TSH I-TSH 6), let alone an optimal solution, for a small problem reinforced the need for developing efficient heuristics for solving real problems encountered in industry practice. Saidi-Mehrabad M. [70] presented a TS algorithm that composed of two parts: a procedure that searched for the best sequence of job operations, and a procedure that found the best choice of machine alternatives. These two parts were used to model FJSSP using meta-heuristics. Computational results indicated that the proposed algorithm could produce optimal solutions in a short computational time for small and medium sized problems. Moreover, it could be applied easily in real factory conditions and for large size problems. For multi-objective FJSSP, Li JQ [71,72] proposed two TS algorithms, HTS and TSPCB, for solving FJSSP. HTS combined with two adaptive rules. A left-shift decoding method was employed to transform a solution to an active schedule. TSPCB combined with critical block neighborhood structure. Insert and swap neighbor structures were used on a critical path to perform local search in HTS and TSPCB algorithms. The two algorithms were tested on sets of benchmark instances and compared to several existing algorithms. Vilcot G [29] proposed two TS algorithms for multi-objective FJSSP. The first was based on the minimization of one criterion subject to a bound on the second criterion and the second was based on the minimization of a linear combination of criteria. Jia S [73] proposed a path-relinking tabu search for the multi-objective flexible job shop scheduling problem. A path-relinking heuristic was designed to generate diverse solutions in the most promising areas. An effective dimension-oriented intensification search was employed to find solutions that were located near extreme solutions.

Eshlaghy AT [74] proposed TS based algorithm for flexible job shop manufacturing systems. The algorithm was compared to a hierarchical method. Performance criteria for comparison are "response quality" and "calculation time" in which results of numerical test approved the priority of TS search algorithm in comparison to the hierarchical method. TS is used in some hybrid algorithms for FJSSP. The literature for these hybrid algorithms will be reviewed in hybrid algorithms.

- Estimation of distribution algorithm (EDA) 
To solve FJSSP, Wang L and Wang SY $[75,76,77]$ proposed three EDA based algorithms, bipopulation based EDA algorithm (BEDA), Pareto-based EDA algorithm (PEDA) and an EDA for fuzzy processing time constraints. In BEDA algorithm, two sub-populations were used to adjust the machine assignment and operation sequencing respectively with a splitting criterion and a combination criterion. In PEDA algorithm, the fitness evaluation based on Pareto optimality was employed and a probability model was built with the Pareto superior individuals for estimating the probability distribution of the solution space. Both BEDA and PEDA algorithms employed critical path based local search operations to enhance performance. Triangle fuzzy number and three fuzzy number operations were used to calculate scheduling objective value in the EDA for FJSSP with fuzzy processing time. In addition, Taguchi method was employed to investigate the influence of parameter setting in all the three EDA based algorithms. Three algorithms were tested though comparing to existing algorithms.

- Variable neighborhood search (VNS)

Various neighborhood structures related to machine assignment and operation sequencing problems were used for generating neighboring solutions. Amiri M. [78] proposed a VNS algorithm for FJSSP. To compare the algorithm with previous ones, an extensive computational study on 181 benchmark problems had been conducted. The results obtained from the presented algorithm were quite comparable to those obtained by the compared algorithms. Yazadani M. [79] proposed a parallel variable neighborhood search algorithm (PVNS). Parallelization in this algorithm was based on the application of multiple independent searches increasing the exploration in the search space. The proposed PVNS used various neighborhood structures that carry the responsibility of making changes in machine assignment and operation sequencing for generating neighboring solutions.

To solve FJSSP with sequence-dependent setup times, Bagheri A. [80] proposed an integrated approach based VNS algorithm. In the algorithm, the external loop controlled the stop condition of the algorithm and the internal loop executed the search process. To evaluate the performance of the proposed algorithm, 20 test problems in different sizes were randomly generated. Consequently, computational results and comparisons validated the quality of the proposed approach. Among the above VNS algorithms, systematic changes of neighborhood structure were generated for evading local optimum. The search processes for finding a local or global optimum solution by VNS are very random. This is one of the weaknesses of this algorithm. To remedy this weakness of VNS, Karimi H. [81] combined VNS algorithm with a knowledge module and proposed a knowledge-based VNS (KBVNS). In KBVNS, the VNS part searched the solution space to find good solutions and knowledge module extracted the knowledge of good solution and feed it back to the algorithm. This would make 
the search process more efficient. Computational results of the paper on different size test problems proved the efficiency of KBVNS algorithm for FJS problem.

In addition, Lei DM [82] presented a VNS algorithm for FJSSP with a dual-resource constraint. In the algorithm, the solution to the problem was indicated as a quadruple string of the ordered operations and their resources. Two neighborhood search procedures were sequentially executed to produce new solutions for two sub-problems of the problem, respectively. The search of VNS was restarted from a slightly perturbed version of the current solution by VNS when the determined number of iterations was reached. This VNS was tested on some instances and was compared with methods from existing literature. Computational results showed the significant advantage of VNS on the problem.

- Hybrid algorithm

To improve algorithm performance, many hybrid algorithms based on more than one meta-algorithm were proposed to solve FJSSP. Tanev IT [83] researched a real-world FJSSP, customers' order in factories of plastic injection machines. A hybrid evolutionary algorithm (HEA) was developed in the literature. HEA combined priority-dispatching rules and GA. The results obtained for evolving a schedule of 400 customers' orders on experiment indicated that the business delays in the order of halfan-hour could be achieved. Fattahi P [84] proposed two types of approaches, hierarchical approaches and integrated approaches to solve FJSSP. Two heuristics involving integrated and hierarchical approaches were developed to solve the real size problems. Six different hybrid-searching structures depending on the used searching approach and heuristics were presented in the paper. Frutos M [85] proposed an algorithm based on non-dominated sorting genetic algorithm II (NSGAII) and SA to solve FJSSP.

Roshanaei N [86] proposed a hybrid of artificial immune and SA (AISA) algorithm for larger instances of FJSSP. The AISA was compared to seven existing algorithms and a developed integer linear programming model. An industrial problem in a mould- and die-making shop was used to verify the AISA algorithm. A hybrid genetic algorithm and SA (GASA) was proposed by Shahsavari-Pour N. [87] for multi-objective FJSSP. Contrary to the other methods that assign weights to all objective functions to reduce them to one objective function, in the GASA and during all steps, problems are solved by three objectives. Gao J [88] researched a hybrid genetic algorithm and bottleneck shifting for multi-objective flexible job shop scheduling problems. Gao J [28] also proposed a hybrid genetic and variable neighbor search algorithm to solve FJSSP. The variable neighbor search algorithms were based on criteria path. Xing LN [89] proposed a multi-population interactive co-evolutionary algorithm for FJSSP. In the study, both ant colony optimization and genetic algorithm with different configuration were applied to evolve each population independently. For FJSSP with transportation constraints and bound processing times, Zhang Q [90] proposed a hybrid genetic algorithm with tabu 
search procedure. A hybrid algorithm of gravitational search and colored petri-net (GSPN) was addressed by Barzegar B [91] to solve FJSSP. In GSPN, the flexible job-shop scheduling problem systems were modeled by color Petri net and color Petri net tool. A scheduled job was programmed by GSPN algorithm. The experimental results showed that the proposed method has reasonable performance in comparison with other algorithms. Liouane N [92] described an ACO and tabu search based local search algorithm to solve FJSSP with makespan objective.

To solve multi-objective FJSSP, Xia WJ [93] researched a hybrid optimization based on PSO and SA. The results obtained from the computational study had shown that the proposed algorithm was a viable and effective approach for the multi-objective FJSSP, especially for problems on a large scale. Shao XY [94] proposed a hybrid discrete PSO (DPSO) and SA algorithm for multi-objective FJSSP. Pareto ranking and crowding distance method were incorporated to identify the fitness of particles in the algorithm. SA was used as a local search algorithm in the above two hybrid algorithms. In addition, to solve multi-objective FJSSP, Zhang GH [53] proposed a hybrid PSO and tabu search algorithm. Three above PSO based hybrid algorithm were proved for multi-objective FJSSP, especially for the problems on a large scale.

Araghi MET [95] proposed an incorporating learning hybrid meta-heuristic based on GA algorithm and VNS method for FJSSP. An affinity function was used to enhance algorithm performance. Taguchi's robust design method was employed to define the best parameter values. Some experiments were designed where the proposed method was compared against some new and successful GA and VNS algorithms. Sadrzadeh A [96] developed an artificial immune system (AIS) and PSO algorithm for scheduling FJSSP. The algorithms were compared to the existing GA algorithm and PVNS. The obtained results showed that the proposed PSO outperforms the GA and the PVNS approaches. It was found that the average best-so-far solutions obtained from the proposed AIS were better than those produced by the GA, the PVNS, the VNS, and the PSO algorithms for all the examples. To solve FJSSP with parallel machine and maintenance constraints, Dalfard VM [97] developed two metaheuristics based on GA and SA. The two meta-heuristics were compared to software LINGO scheduling system. The results showed that the applied hybrid GA and SA algorithms were much more effective than the solutions obtained using LINGO. Finally, solutions using the simulated annealing approach were compared with solutions of the hybrid genetic algorithm.

- Others algorithms

There are some other algorithms for FJSSP. To solve FJSSP, Wu ZB [98] proposed a multiagent scheduling method with earliness and tardiness objectives. A job-routing and sequencing mechanism was proposed. In the mechanism, two kinds of jobs were defined to distinguish jobs with one operation left from jobs with more than one operation left. Different criteria were proposed to route these two 
kinds of jobs. Job sequencing enables to hold a job that might be completed. Two heuristic algorithms for job sequencing were developed to deal with these two kinds of jobs. The computational experiments showed that the proposed multi-agent scheduling method significantly outperforms the existing scheduling methods in the literature.

Yu XF [99] addressed a bio-inspired multi-agent model for FJSSP with sequence dependent setups. The study set the model parameters using two steps, termed mapping and tuning. Mapping established a set of coefficients to link the model parameters with the scheduling problem characteristics. Tuning determined good values of these coefficients were used to compute the model parameters. The performance of the proposed multi-agent model was compared with another scheduling method that was based on dispatching rules. It was concluded that the proposed parameter setting method was effective and worth considering when applying bio-inspired division of labor to dynamic manufacturing scheduling.

To solve FJSSP with alternative process plans, Rajabinasab A [100] proposed an agent based scheduling system. A pheromone-based approach was proposed for coordination among agents. The approach was compared with five dispatching rules from literature via simulation experiments to statistical analysis. The simulation experiments were performed under various experimental settings such as shop utilization level, due date tightness, breakdown level, and mean time to repair. To solve FJSSP with machine availability constraints, Moradi E [101] established an architecture. The literature applied an enforceable and easily extendable preventive maintenance policy on FJSSP. In order to figure the problem out, all operators and parameters were calibrated by means of the Taguchi experimental design.

Baghei A [102] addressed an artificial immune algorithm (AIA) for FJSSP. AIA used several strategies for generating the initial population and selecting the individuals for reproduction. Different mutation operators were utilized for reproducing new individuals. To show the effectiveness of AIA, numerical experiments by using benchmark problems were conducted. Consequently, the computational results validated the quality of AIA.

Karthikeyan S. [103] proposed a discrete firefly algorithm for multi-objective FJSSP. The machine assignment and operation sequence were processed by constructing a suitable conversion of the continuous functions as attractiveness, distance and movement, into new discrete functions. Meanwhile, local search method with neighborhood structures is hybridized to enhance the exploitation capability.

A hybrid SFLA algorithm was proposed by Li JQ [104] to solve multi-objective FJSSP. In the algorithm, several approaches were presented to construct the initial population with a high level of quality. Two crossover operators were presented to share information among the best frogs and the 
worst frog. Several local search methods were embedded in the algorithm to enhance the exploitation capability. Li JQ [105] also proposed a discrete chemical-reaction optimization (DCRO) for FJSSP with maintenance activity. In the DCRO, each solution was represented by a chemical molecule. Four improved elementary reactions, on-wall ineffective collision, inter-molecular ineffective collision, decomposition, and synthesis, were developed. A well-designed crossover function was introduced in the inter-molecular collision, synthesis, and decomposition operators. In addition, the decoding mechanism considering the maintenance activity was presented. Several neighboring approaches were developed to improve the local search ability of the DCRO. Through the analysis of experimental results, the highly effective performance of the proposed DCRO algorithm was shown.

Rajkumar M [106,107] proposed two greedy randomized adaptive search procedure (GRASP) to solve FJSSP with maintenance constraints and limited resource constraints. The two GRASP algorithms were meta-heuristic algorithms that were characterized by multiple initializations. It operated in the following manner: first a feasible solution was obtained, which was then further improved by a local search technique. Representative benchmark problems were solved in order to test the effectiveness and efficiency of the two GRASP algorithms.

Farughi H [108] proposed a memetic algorithm (MA) for FJSSP with overlapping operations. A heuristic used the critical path method (CPM) in order to improve the results of MA and reduce the objective function. The experimental results showed that MA was capable of achieving the optimal solution for small size problems and near-optimal solutions for medium and large size problems in a reasonable time.

In addition, Nie L [109] studied a heuristic to implement reactive scheduling for the dynamic scheduling problem. A gene expression programming (GEP) algorithm was developed for scheduling FJSSP with dynamic job releasing date constraint. The GEP automatically constructed reactive scheduling policies for the dynamic scheduling. A variety of processing conditions three factors, such as the shop utilization, due date tightness, problem flexibility, were considered in the simulation experiments. The results showed that GEP-based approach could construct more efficient reactive scheduling policies for DFJSSP with job release dates under a big range of processing conditions. Rahmati SHA [110] proposed a Biogeography-based optimization (BBO) algorithm for FJSSP. The migration operators of BBO were developed for searching a solution area of FJSSP and finding the optimum or near-optimum solution to this problem. To assess the performance of BBO, it was compared with a genetic algorithm that had the most similarity with the proposed BBO. This similarity caused the impact of different neighborhood structures being minimized and the differences among the algorithms being just due to their search quality. To evaluate the distinctions of the two algorithms 
much more elaborately, they were implemented on three different objectives. BBO was also compared with some famous algorithms in the literature.

\subsection{Harmony search algorithm}

Harmony search (HS) is one of the recent meta-heuristic methods presented by Geem et al [111] for solving optimization problems. It imitates the improvisation process of musicians. The harmony in music is analogous to the solution vector in the corresponding optimization problem, and the musician's improvisations are analogous to local and global search in optimization techniques. The harmony search algorithm generates a new candidate solution from all existing solutions. Compared to the earlier meta-heuristics, the HS algorithm imposes fewer mathematical requirements. In addition, numerical comparisons show that the process of evolution in harmony algorithm is faster than that of the GA [112]. The authors of the literature improved the HS performance. They changed the parameters pitch adjusting rate (PAR) and bandwidth (bw) for each generation from fix values to dynamic values with different generations. The results for benchmark instance and standard engineering optimization problems show that harmony search can find better solutions when compared to other heuristics or deterministic methods. Harmony search algorithm is also a powerful search algorithm for various engineering optimization problems.

The computational procedure of the basic single objective HS algorithm can be summarized as follows: Step 1: Initialization

The harmony vector or solution is generally represented as an $n$-dimensional real-valued vector $X=\{x(1), x(2), \ldots, x(n)\}$, where each dimension $x(k)$ denotes a decision variable of the optimization problem. In this step, the aims are to set parameters and fill the harmony memory ( $H M)$ with a population of initial harmonies (solutions). Parameters include the harmony memory size ( $H M S$ ), harmony memory consideration rate ( HMCR), pitch adjustment rate $(P A R)$ and distance bandwidth $(B W)$. The HM is filled by randomly generated harmony vectors.

Let $X_{i}=\left\{x_{i}(1), x_{i}(2), . ., x_{i}(n)\right\}$ represent the $i^{\text {th }}$ harmony vector in the HM, which is generated as follows:

$$
x_{i}(j)=L B(j)+(U B(j)-L B(j)) \times \operatorname{rand}(0
$$

for $j=1,2, . ., n$ and $i=1,2, \cdots H M S$

where $\operatorname{rand}()$ is a uniform random number in the range of $[0,1], L B(j)$ and $U B(j)$ are the lower and upper bounds for the decision variable $x(j)$, respectively.

Step 2: Improvising a new harmony 
The process to produce a harmony vector $X_{n e w}$ is called improvisation. $X_{n e w}$ is produced by applying three rules, i.e., a memory consideration, a pitch adjustment and a random selection. First of all, if a uniform random number $\operatorname{rand}(0)$ in the range of $[0,1]$ is less than $H M C R$, the decision variable $x_{\text {new }}(j)$ is generated by the memory consideration, otherwise, $x_{\text {new }}(j)$ is obtained by a random selection. Secondly, each decision variable $x_{\text {new }}(j)$ will undergo a pitch adjustment with a probability of PAR if it is updated by the memory consideration. The memory consideration, pitch adjustment and random selection are given in Eqs (3-2), (3-3) and (3-4), respectively.

$$
\begin{aligned}
& \text { if }(\text { rand }()<H M C R) \\
& \qquad x_{\text {new }}(j)=x_{a}(j) \\
& \quad i f(\operatorname{rand} 2()<P A R) \\
& \quad x_{\text {new }}(j)=x_{\text {new }}(j) \pm \operatorname{rand}() \times B W \\
& \text { else } \\
& \quad x_{\text {new }}(j)=L B(j)+\operatorname{rand}() \times(U B(j)-L B(j))
\end{aligned}
$$

where $a \in(1,2, \ldots, H M S), x_{a}(j)$ is randomly selected from HM.

Step 3: Updating the HM

The HM will be updated if $X_{n e w}$ is better than the worst harmony vector $X_{w}$ in the HM. In this case, $X_{n e w}$ will replace $X_{w}$ and become a new member of the HM.

Step 4: Stopping condition

If a termination criterion is met, return the best harmony vector $X_{B}$ in the HM; otherwise go to Step

2.

Many researchers have developed HS algorithm and have applied HS variants to solve industry optimization problems [113]. The authors of the literature employed HS algorithm for solving discrete structural optimization problem. The numerical results showed HS could yield better solutions than those obtained by existing methods. Omran and Mahdavi [114] developed a global-best harmony search algorithm. In the paper, the authors modified the pitch-adjustment steps so that the new harmony can mimic the best harmony in harmony memory. The BW parameter was replaced altogether and added a social dimension. The global-best HS algorithm could work efficiently on both continuous and discrete problems. Das [115] analyzed and improved the exploration power of HS algorithm. These papers analyzed the evolution of the population-variance over successive generations in HS and draw some important conclusions regarding the explorative power of HS. Geem applied harmony search algorithm for scheduling multiple dam system [116]. HS algorithm tackled popular 
benchmark system with four dams. The HS algorithm could find more global optimal solutions than the compared GA algorithm in the literature. Furthermore, HS model arrived at the global optima without any sensitivity analysis of algorithm parameters whereas the compared GA model required tedious sensitivity analysis.

In recent years, HS are also employed for solving shop scheduling problem. Wang [117] and Pan [118] proposed a hybrid harmony search algorithm and a local-best harmony search algorithm with dynamic sub-harmony memories for blocking flow shop scheduling problem and lot-streaming flow shop scheduling problem. Gao proposed a grouping harmony search algorithm [119] and a discrete harmony search (DHS) algorithm [120] for no-wait flow shop scheduling problem. In these shop scheduling problem papers, some improving strategies for HS were developed, for example, sub-harmony memory for convergence of HS, local-best search for exploration. Based on the shop scheduling problem features, some simple heuristics or dispatch rules were employed in HS algorithm to improve the quality of initial solutions, for example, NEH heuristic. To improve the local search performance, HS is mixed with several local search operators that are famous in shop scheduling problem, for example insert operator, exchange operator and reverse operator etc.

To solve FJSSP and large-scale FJSSP, Yuan Y [121,122] proposed two harmony search based algorithms. In the proposed HS algorithms, HS was converted to adapt to FJSSP. Local search based on common critical operation was developed to enhance performance. For the large-scale FJSSP, large neighborhood search was integrated to HS to improve results. The HS algorithm for large-scale FJSSP had even found some new upper bounds among the solved benchmark instances. The author of this thesis proposed a discrete harmony search algorithm (DHS) [25] and a Pareto-based grouping discrete harmony search (PGDHS) algorithm [123]. A proposed heuristic and several existing simple heuristics were employed to initialize harmony memory in the two algorithms. A new rule was developed for improvisation a new harmony. In PGDHS algorithm, dynamic grouping was used to improve population diversity. Three widely used performance measures, a number of non-dominated solutions, diversification metric and quality metric, were employed to test the performance of PGDHS algorithm.

\subsection{Artificial bee colony}

Artificial Bee Colony (ABC) is one of the most recently defined algorithms by Dervis Karaboga in 2005, motivated by the intelligent behavior of honey bees [124]. It is simple and uses only common control parameters such as colony size and maximum cycle number. $\mathrm{ABC}$ as an optimization tool provides a population-based search procedure in which individuals are called foods positions and are modified by the artificial bees with time. In ABC, the colony of artificial bees contains three groups 
of bees: employed bees associated with specific food sources, onlooker bees watching the dance of employed bees within the hive to choose a food source, and scout bees searching for food sources randomly. The bee's aim is to discover the places of food sources with high nectar amount and to find the one with the highest nectar. In $\mathrm{ABC}$ system, artificial bees fly around in a multidimensional search space and some (employed and onlooker bees) choose food sources depending on the experience of themselves and their nest mates, and adjust their positions. Some (scouts) fly and choose the food sources randomly without using experience. If the nectar amount of a new source is higher than that of the previous one in their memory, they memorize the new position and forget the previous one. Thus, ABC system combines local search methods, carried out by employed and onlooker bees, with global search methods, managed by onlookers and scouts, attempting to balance exploration and exploitation process.

The main steps of the basic $\mathrm{ABC}$ algorithm are as followings.

1) Initialization of the parameters and population:

The parameters of $\mathrm{ABC}$ are the number of food sources ( $S N$ ), the number of trials after which a food source is to be abandoned (limit) and the termination criterion. The number of food sources is equal to the number of employed bees or onlooker bees. The initialization of population is to fill the population with $S N$ number of randomly generated food sources, $n$-dimensional realvalued vectors. Let $X_{i}=\left\{x_{i 1}, x_{i 2}, \ldots, x_{i n}\right\}$ represent the $i$ th food source in the population. The food sources are generated as follows:

$$
x_{i j}=L B_{j}+\left(U B_{j}-L B_{j}\right) \times r \text { for } j=1,2, \ldots, n \text { and } i=1,2, \ldots, S N
$$

where $r$ is a uniform random number in the range $[0,1] ; L B_{j}$ and $U B_{j}$ are the lower and upper bounds for the dimension $j$, respectively. The food sources are randomly assigned to employed bees and the corresponding fitnesses are evaluated.

2) Employed bee phase:

In this phase, each employed bee $X_{i}$ generates a new food source $X_{n e w}$ in the neighborhood of its present position as follows:

$$
x_{n e w(j)}=x_{i j}+\left(x_{i j}-x_{k j}\right) \times r^{\prime}
$$

where $k \in\{1,2, \ldots, S N\} \wedge k \neq i$ and $j \in\{1,2, \ldots, n\}$ are randomly chosen indexes. $r^{\prime}$ is a uniformly distributed real number in $[-1,1]$.

$X_{n e w}$ will be compared to $X_{i}$. If the fitness of $X_{n e w}$ is equal or better than that of $X_{i}, X_{n e w}$ will replace $X_{i}$ as a new food source; otherwise $X_{i}$ is retained. 
3) Onlooker bee phase:

An onlooker bee evaluates all the employed bees and selects a food source $X_{i}$ depending on its probability value $p_{i}$ calculated by the following expression.

$$
p_{i}=\frac{f_{i}}{\sum_{i=1}^{S N} f_{i}}
$$

where $f_{i}$ is the nectar amount or the fitness value of the $i$ th food source $X_{i}$. The higher the $f_{i}$ is, the more ability that the $i$ th food source is selected.

Once the food source $X_{i}$ is selected, the onlooker bee will execute the update $X_{i}$ using equation (3). If the new food source has equal or better fitness value than $X_{i}$, the new food source will replace $X_{i}$ as a new member in the population.

4) Scout bee phase:

If a food source $X_{i}$ can not be improved through a predetermined number of trials limit, the food source is to be abandoned and the corresponding employed bee becomes a scout. The scout produces a new food source randomly as follows:

$$
x_{i j}=L B_{j}+\left(U B_{j}-L B_{j}\right) \times r \text { for } j=1,2, \ldots, n
$$

where $r$ is a uniform random number in the range $[0,1]$.

5) Repeat steps 2)-4) until the termination condition is satisfied.

In recent years, $\mathrm{ABC}$ algorithm has received intensive interest from researchers in a variety of fields. It was first proposed to solve the multi-variable and multi-modal continuous functions [124]. Many comparative studies have shown that the performance of the $\mathrm{ABC}$ algorithm is competitive to other population-based algorithms with the advantage of employing fewer control parameters in the continuous space $[125,126,127,128]$. Banharnsakun, Achalakul and Sirinaovakul adopted the bestso-far selection in $\mathrm{ABC}$ to enhance the exploitation and exploration processes [129].

$\mathrm{ABC}$ is used to solve combinatorial optimization problems. Wong, Low, and Chong developed a bee colony optimization with a local search to solve the travelling salesman problem [130]. For shop scheduling problem, a discrete artificial bee colony algorithm based on a self-adaptive strategy was proposed to solve the lot-streaming flow-shop scheduling problem with the criterion of total weighted earliness and tardiness penalties [131]. Banharnsakun, Sirinaovakul, and Achalakul applied the bestso-far ABC (B-ABC) to solve the JSSP problem [132].

In recent years, ABC algorithm is developed for FJSSP by many researchers. Li JQ [26,133] proposed an $\mathrm{ABC}$ algorithm and a Pareto-based discrete $\mathrm{ABC}$ algorithm for multi-objective FJSSP. Pareto archive set was employed to record non-dominated solutions in the two algorithms. Several local 
search approaches were designed to balance the exploration and exploitation capability of the algorithm in discrete ABC algorithm. Li JQ [134] also proposed an artificial bee colony algorithm for FJSSP with maintenance activities constraint. An efficient initialization scheme was introduced to construct the initial population with a certain level of quality and diversity. A self-adaptive strategy was adopted to enable the DABC algorithm with learning ability for producing neighboring solutions in different promising regions whereas an external Pareto archive set was designed to record the nondominated solutions found so far.

Two effective ABC algorithms were presented by Wang L $[135,136]$ to solve the mono-objective and the multi-objective FJSSP respectively. The first ABC algorithm was for makespan objective while the second one was for Pareto-based multi-objective. Multiple initializing strategies and critical path based local search are employed to improve algorithms' performance. In addition, Wang L [137] also proposed an ABC algorithm for FJSSP with fuzzy processing time. In the algorithm, initializing strategies, left-shift decoding scheme and local search based on variable neighbor search were employed to enhance performance. Taguchi method was used to investigate the influence of several key parameters in the three algorithms proposed.

\subsection{Shortcomings and research trends}

Most existing research focuses on developing meta-heuristics for general FJSSP and few papers address on the practical application in variety industry environments. There are some problems and new trends for FJSSP.

1. The practical constraints in variety environments are serious obstacles to apply the meta-heuristics to FJSSP in the real shop floor. How to solve FJSSP with constraints in variety industry environments is a new trend of FJSSP research.

2. Most existing scheduling methods and algorithms cannot satisfy the dynamic changes of resources, environments and other factors in the real shop floor. Dynamic rescheduling is needed to satisfy the changes in practical production environments.

3. Little existing literature applies meta-heuristics to remanufacturing related scheduling problems and deal with the scheduling related constraints in remanufacturing.

Based on the review in Sections 2.3 and 2.4, harmony search algorithm and artificial bee colony algorithm have been successfully applied to a variety of engineering optimization problems. The effectiveness and efficiency of these two algorithm are also been proved in many papers. In this thesis, we employ discrete harmony search algorithm and artificial bee colony algorithm to solve scheduling problem in the remanufacturing industry. Two scheduling related constraints in remanufacturing 
environment are solved to satisfy the practical requirements in the real shop floor. The most probable processing time and fuzzy number are employed to represent the uncertain processing time of operations in remanufacturing industry. Dynamic rescheduling is developed in this thesis for new job insertion constraint in remanufacturing. 


\section{Chapter 3}

\section{Modelling FJSSP with Multiple Constraints}

In this chapter, the FJSSP model is described in detail and two characteristics in the remanufacturing environment, new job insertion and uncertain or fuzzy processing time, are modeled as constraints of FJSSP.

\subsection{Flexible job shop scheduling problem}

In a flexible job shop, each job consists of a sequence of operations. An operation can be executed on only one machine out of a set of candidate machines. Each operation of a job must be processed only on one machine at a time, while each machine can process only one operation at a time.

The following notations and assumptions are used for the formulation of multi-objective FJSSP.

1. Let $J=\left\{J_{i}\right\}, 1 \leq i \leq n$, index $i$, be a set of $n$ jobs to be scheduled. $q_{i}$ denotes the total number of operations of a job $J_{i}, d_{i}$ is the due date of $J_{i}$.

2. Let $M=\left\{M_{k}\right\}, 1 \leq k \leq m$, index $k$, be a set of $m$ machines.

3. Each job $\boldsymbol{J}_{i}$ consists of a predetermined sequence of operations. Let $O_{i, h}$ be operation $h$ of $\boldsymbol{J}_{i}$.

4. Each operation $O_{i, h}$ can be processed without interruption on one of the set of candidate machines $M\left(O_{i, h}\right)$. Let $P_{i, h, k}$ be the processing time of $O_{i, h}$ on machine $M_{k}$. 
5. Decision variables

$$
x_{i, h, k}=\left\{\begin{array}{l}
1, \text { if machine } k \text { is selected for the operation } O_{i, h} \\
0, \text { otherwise }
\end{array}\right.
$$

$c_{i, h}$ denotes the completion time of the operation $O_{i, h}$

$c_{i}$ denotes the completion time of the job $J_{i}$

6. The objectives considered in this thesis are as follows:

Makespan, denoted by $C_{M}$, is the maximal of completion time of machines.

$$
\operatorname{Min} C_{M}=\max _{1 \leq i \leq n} c_{i}
$$

Mean of earliness and tardiness, denoted by $E / T$, is the average of all jobs' earliness or tardiness compared to their due dates.

$$
\operatorname{Min} E / T=\frac{\sum_{i=1}^{n}\left|c_{i}-d_{i}\right|}{n}
$$

where $C_{i}$ is the completion time of job $J_{i}$ and $d_{i}$ is the due date of job $J_{i}$.

Maximum machine workload, denoted by $W_{M}$, can be calculated by:

$$
\operatorname{Min} W_{M}=\max _{1 \leq j \leq m} w_{j}
$$

Where $w_{j}$ is the workload of machine $M_{j}$

The FJSP is a NP-complete problem when the mahine number is laerger than two [13]. The time completity of calculation for objective is about $\boldsymbol{O}\left(n^{3} \mathrm{~m}^{2}(\log O)^{2}\right)$, where $n$ is the job number, $m$ is the machine number and $O$ is the total operation number of all jobs.

\subsection{New job insertion constraint}

In remanufacturing environment, the new job arrival is unpredictable. When a new job arrives and is inserted into the processing job sequence, conflicts may arise and the objective may be affected. Rescheduling is needed in this condition. Because the scheduling solution is executing in workshop, the machines are available for rescheduling when the current operations on them are completed. It means that machines and jobs may have different available times when rescheduling is performed. We model the new job arrival and insertion feature of remanufacturing as a constraint of FJSSP, New job Insertion.

To explain FJSSP with a new job insertion, an example is shown in the following content. Fig. 3 - 1(a) shows a Gantt chart for 3-jobs and 3-machines FJSSP. The numbers of operations in three jobs are $J_{1}$, $3, J_{2}, 2$ and $J_{3}, 2$, respectively. The makespan value in this scheduling solution is 10 . The completion time of three machines is $M_{1}, 8, M_{2}, 7$ and $M_{3}, 10$. Fig. 3 - 1(b) shows the new job, $J_{4}$, arrives and is 
inserted into the current running schedule at Time 3. The job $J_{4}$ has three operations. Fig. 3 - 1(c) shows the result with no rescheduling. The existing schedule remains the same and the Job4 is scheduled after all the assigned operation on the machines. The earliest start time of the three machines for rescheduling is $M_{1}, 8, M_{2}, 7$ and $M_{3}, 10$. Fig. 3 - 1(d) shows the revised schedule after rescheduling. Both new $J_{4}$ and all yet-to-start operations of existing three jobs are rescheduled. The earliest available time of $M_{1}$ and $M_{3}$ is 3 while the available time of jobs $J_{2}, J_{3}$ and $J_{4}$ is also 3. $M_{2}$ is processing the first operation of Job1 when the Job4 inserts at Time 3. $M_{2}$ and $J_{1}$ are available when the first operation of $J_{1}$ is completed. Therefore, the available time of $M_{2}$ and $J_{1}$ is Time 4 . In Fig. 3 - 1(d), the start time of $O_{1,2}$ and $O_{1,3}$ are delayed as compared to that of schedule in Fig. 3 - 1(b).
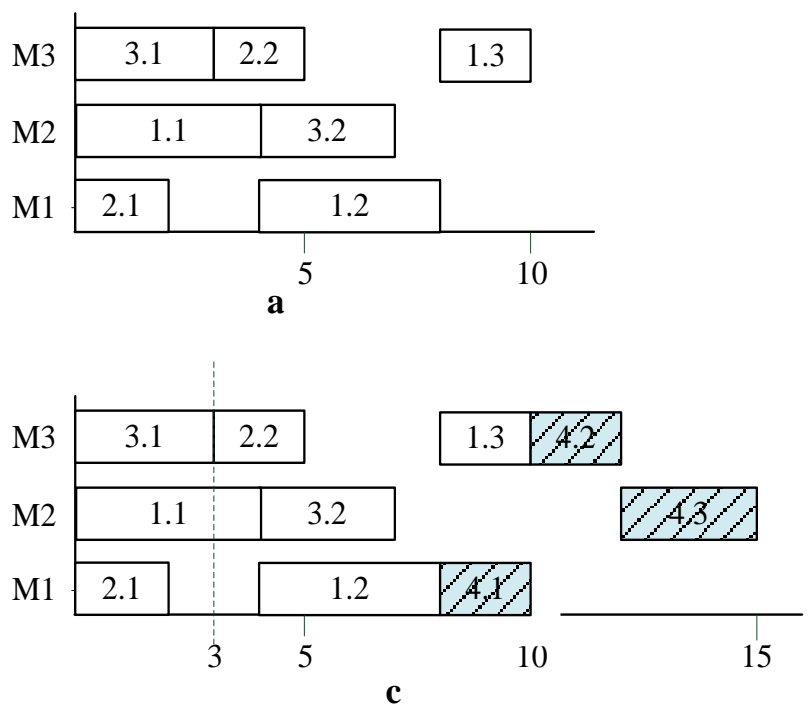
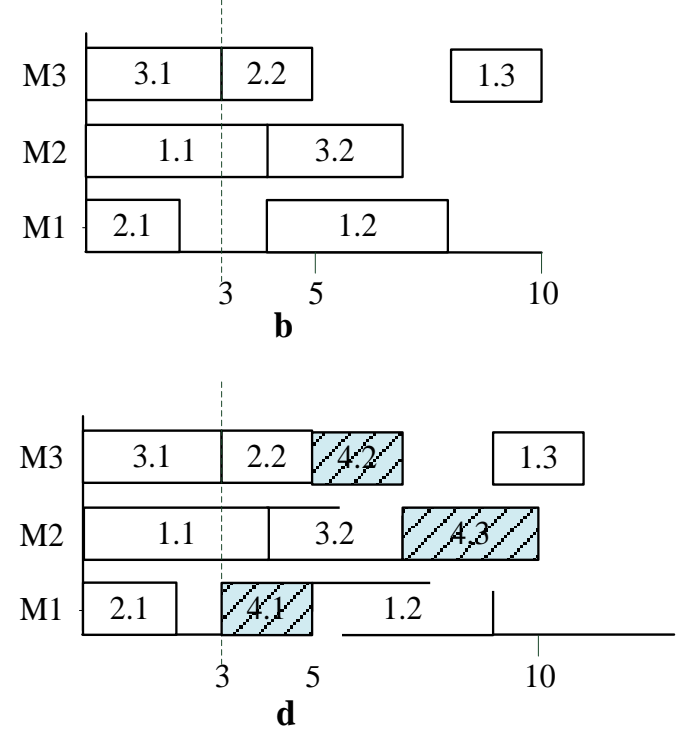

Fig. 3 - 1 An example of new job insertion

\subsection{Uncertain processing time constraint}

In remanufacturing environment, the operation processing time is not fixed and may vary in real shop floor. This section proposed two strategies to model the uncertainty of processing time. One model is based on the most probable processing time and another model is based on fuzzy number.

\subsubsection{Modeling based on most probable processing time}

In this model, the scheduling is according to operation's most probable processing time that is evaluated by engineers' experiences. Rescheduling is executed when the operation processing time increases larger than the most probable processing time. To explain the model detail, an example is shown in following contents. 
Table 3 - 1 shows the operation most probable processing time of job re-processing in remanufacturing. There are three Volutes and two Impellers jobs. The first operation of Volute 2 can only be processed by milling machine 1 . The processing time is 2 . For the two Impeller jobs, there are different selectable machines and the processing times on different machines are different.

Table 3 - 1 An example of the model based on the most probable processing time

\begin{tabular}{|c|c|c|c|c|c|}
\hline \multirow{2}{*}{$\begin{array}{c}\text { Sub- } \\
\text { assembly }\end{array}$} & \multicolumn{2}{|l|}{ O 1} & \multirow{2}{*}{$\begin{array}{c}\text { O } 2 \\
\text { Metal building }\end{array}$} & \multicolumn{2}{|l|}{ O 3} \\
\hline & Milling 1 & Milling 2 & & Turning 1 & turning 2 \\
\hline Volute 1 & 5 & 4 & 6 & 5 & 4 \\
\hline 2 & 2 & & 8 & 4 & 3 \\
\hline 3 & 5 & 4 & 8 & 11 & 7 \\
\hline Impeller 1 & 5 & 4 & 6 & 4 & 2 \\
\hline 2 & 3 & 6 & & 3 & 4 \\
\hline
\end{tabular}
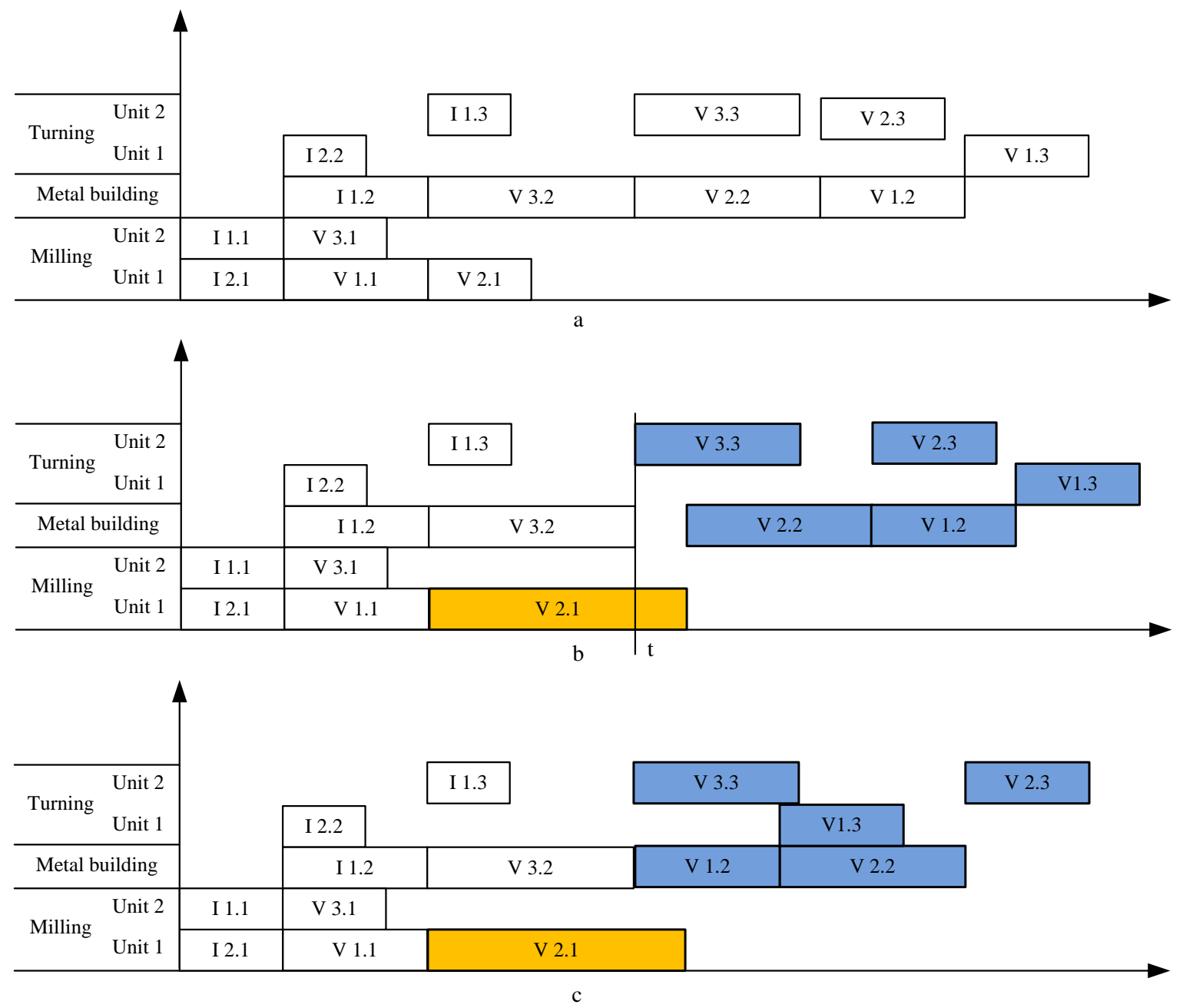

Fig. 3 - 2 An example of increasing processing time 
Fig. 3 - 2 (a) shows one scheduling solution of the data in Table 3 - 1. If the processing time increases and affects the objective, rescheduling is executed on the operations that are not start yet in order to improve the results. For example, the processing time of operation V2.1 on machine milling 2 increases from the value shown in Fig. 3 - 2 (a) to that shown in Fig. 3 - 2 (b). The processing time change of job operation V2.1 affects the maximum completion time after time " $t$ ". The operations after V2.1 should be rescheduled at time "t". These operations are V2.2, V2.3, V1.2, V1.3 and V3.3. Fig. 3 - 2 (c) shows the Gantt chart after rescheduling. The operations V1.2 and V2.2 exchange their positions. The start time of operations V1.2 and V1.3 becomes earlier than that in Fig. 3 - 2 (b). It is clear that the maximum complete time in Fig. 3 - 2 (c) is less than that in Fig. 3 - 2 (b).

\subsubsection{Modeling based on triangular fuzzy number}

In this model, we model the uncertain processing time as fuzzy processing time. The uncertainty processing time is described in three values, the smallest processing time, the most probable processing time and the largest processing time. Triangular fuzzy number (TFN) is used to show the operation processing time in scheduling process. The TFN is shown in the following formula:

$$
t_{i, j, k}=\left(t_{i, j, k}^{1}, t_{i, j, k}^{2}, t_{i, j, k}^{3}\right)
$$

where $t_{i, j, k}^{1}, t_{i, j, k}^{2}$ and $t_{i, j, k}^{2}$ are three probable processing time of operation $\boldsymbol{O}_{i, j}$ on machine $M_{k}$.

To compare and order TFN, addition operation, maximum operation and ranking operation are used. The addition operation is for computing the summary time. The maximum operation and the ranking operation are used to compare TFNs. The three operations are computed as follows:

Addition operation: two TFNs, $t=\left(t_{1}, t_{2}, t_{3}\right)$ and $t^{\prime}=\left(t_{1}^{\prime}, t_{2}^{\prime}, t_{3}^{\prime}\right)$, their addition is as follows.

$$
t+t^{\prime}=\left(t_{1}+t_{1}^{\prime}, t_{2}+t_{2}^{\prime}, t_{3}+t_{3}^{\prime}\right)
$$

Ranking operation: three criteria are used to compare two TFNs $t=\left(t_{1}, t_{2}, t_{3}\right)$ and $t^{\prime}=\left(t_{1}^{\prime}, t_{2}^{\prime}, t_{3}^{\prime}\right)$.

1. If $\left(t_{1}+2 t_{2}+t_{3}\right) / 4>(<)\left(t_{1}^{\prime}+2 t_{2}^{\prime}+t_{3}^{\prime}\right) / 4$, then $t>(<) t^{\prime}$.

2. If $\left(t_{1}+2 t_{2}+t_{3}\right) / 4=\left(t_{1}^{\prime}+2 t_{2}^{\prime}+t_{3}^{\prime}\right) / 4$, then $t_{2}$ and $t_{2}^{\prime}$ is compared. If $t_{2}>(<) t_{2}^{\prime}$, then $t>(<) t^{\prime}$.

3. If $t_{2}=t_{2}^{\prime}$, then the spreads of two TFNs are compared. If $t_{3}-t_{1}>(<) t_{3}^{\prime}-t_{1}^{\prime}$, then $t>(<) t^{\prime}$.

Maximum operation: The max of two TFNs is approximated with the criterion: If $t>t^{\prime}$, then $t \vee t^{\prime}=t$; otherwise $t \vee t^{\prime}=t^{\prime}$.

To explain the model in detail, an example is shown. Table 3 - 2 shows the fuzzy processing time of 3 jobs on 4 machines. In Table 3 - 2, each fuzzy processing time includes three values. For example, the processing time of operation $O_{2,1}$ on machine $M_{3}$ is $(1,2,5)$. This means that the smallest 
processing time is " 1 ", the most probable processing time is " 2 " and the largest processing time is " 5 ". To present the scheduling Gantt chart using TFN, Fig. 3 - 3 shows a Gantt chart of one solution for the data in Table 3 - 2.

Table 3 - 2 An example of triangle fuzzy number for modeling uncertain processing time

\begin{tabular}{cccccc}
\hline \multirow{2}{*}{ Job } & \multirow{2}{*}{ Operation } & \multicolumn{5}{c}{ Machine } \\
\cline { 3 - 6 } & & $M_{1}$ & $M_{2}$ & $M_{3}$ & $M_{4}$ \\
\hline \multirow{2}{*}{1} & $O_{11}$ & $(5,6,7)$ & - & $(3,5,6))$ \\
& $O_{12}$ & $(1,2,3)$ & $(6,8,10)$ & $(5,9,11)$ & $(9,10,14)$ \\
& $O_{13}$ & $(1,3,4)$ & - & $(4,6,8)$ & - \\
2 & $O_{21}$ & - & $(5,7,9)$ & $(1,2,5)$ & $(1,2,3)$ \\
& $O_{22}$ & $(5,7,9)$ & $(4,5,7)$ & $(3,5,6)$ & $(1,2,3)$ \\
& $O_{23}$ & $(7,8,11)$ & - & - & $(1,3,4)$ \\
& $O_{24}$ & $(7,9,10)$ & - & $(5,6,9)$ & - \\
3 & $O_{31}$ & $(2,4,8)$ & - & $(4,5,7)$ & - \\
& $O_{32}$ & $(6,8,12)$ & $(5,8,10)$ & $(6,7,10)$ & $(4,7,9)$ \\
& $O_{33}$ & $(8,9,12)$ & $(3,4,5)$ & - & - \\
\hline
\end{tabular}

In Fig. 3 - 3, the triangle under machine line means the start time of one operation while the triangle above the machine line means the completion time of one operation. From the left side, the first vertex of one triangle means the smallest start or completion time. The second vertex means the most probable start or completion time and the last vertex presents the largest start or completion time. For example, the first triangle above the line of $M_{4}$ means the completion time of operation $O_{1,1}$ is $(3,5,6)$. From the left, the first vertex value is " 3 ", the second vertex value is " 5 " and the third vertex value is "6". The first triangle under the line of machine $M_{4}$ means the start time of operation $O_{2,3}$ is $(7,11$, 17). For each job, if the first operation starts from time 0 , the start time is presented using a rectangle under the line of processing machine.

To explain the Gantt chart in detail, job 2 is as an example. The first operation $O_{2,1}$ is processed on machine $M_{3}$. The start time of operation $O_{2,1}$ is 0 . There is a rectangle under machine $M_{3}$. The completion time of operation $O_{2,1}$ is $(1,2,5)$ and the first triangle above the line of machine $M_{3}$ shows the fuzzy number $(1,2,5)$. From the left, the first vertex value is " 1 ", the second vertex value is " 2 " and the third vertex value is " 5 ". The operation $O_{2,2}$ is processed on machine $M_{1}$ and the start time is $(2,4,8)$. The first triangle under the line of machine $M_{1}$ shows the fuzzy start time $(2,4,8)$. The completion time of $O_{2,2}$ is $(7,11,17)$ and the second triangle above machine $M_{1}$ line shows the fuzzy number $(7,11,17)$. The operation $O_{2,3}$ is processed on machine $M_{4}$ and the start time is $(7,11,17)$. 
The first triangle under machine $M_{4}$ line shows the fuzzy start time. The completion time of $O_{2,3}$ is (8, $14,21)$ and the second triangle above machine $M_{4}$ line shows the fuzzy completion time. The operation $O_{2,4}$ is processed on machine $M_{3}$ and the start time is $(8,14,21)$. The second triangle under machine $M_{3}$ line shows the fuzzy start time. The completion time of $O_{2,4}$ is $(13,20,30)$ and the third triangle above machine $M_{3}$ line shows the fuzzy completion time.

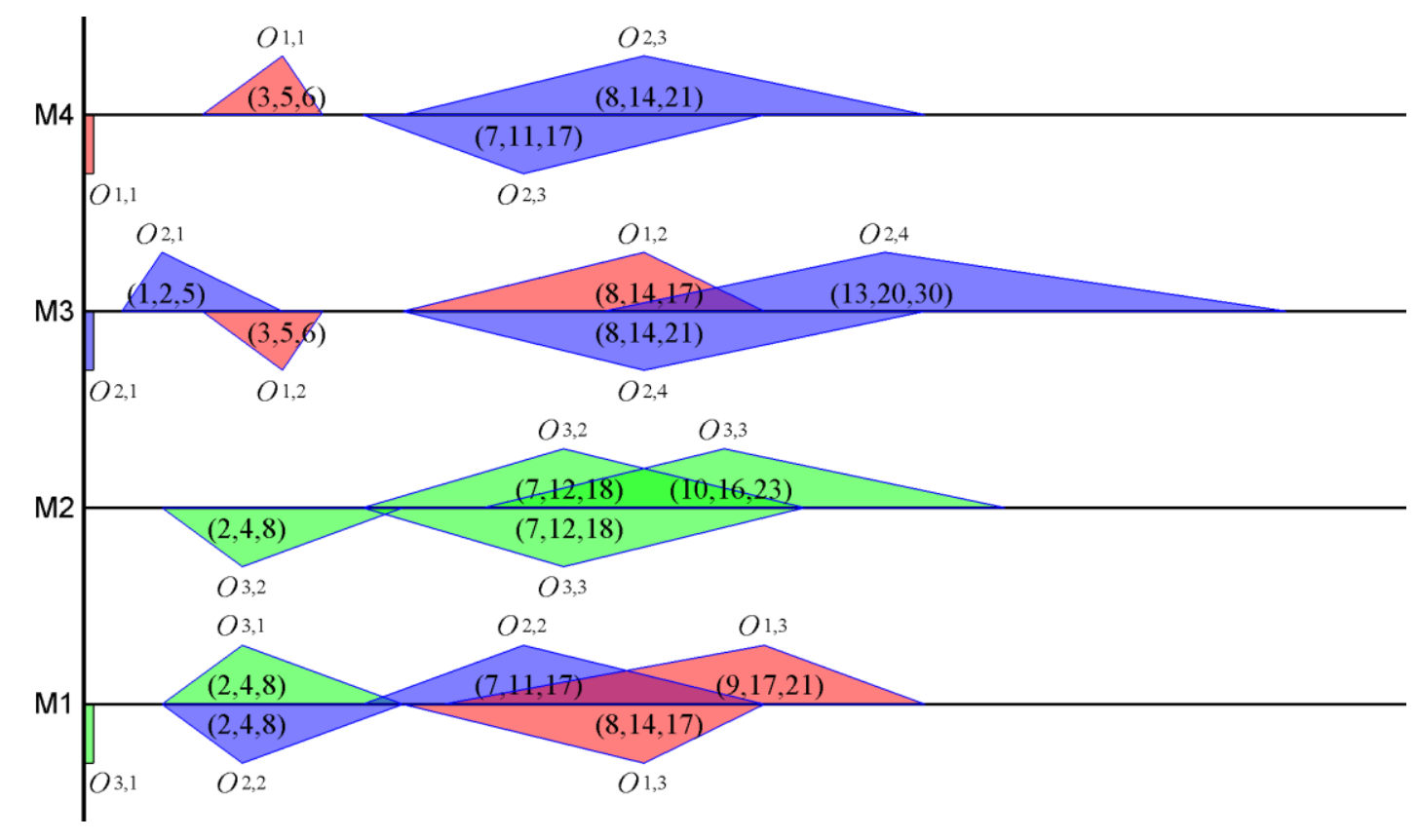

Fig. 3 - 3 Fuzzy Gantt chart

\subsubsection{Comparisons of two models}

Two models for uncertain processing time constraint are proposed in section 3.3.1 and section 3.3.2. The first model is based on the most probable processing time and the second model is based on fuzzy number. The first model can obtain a solution with an exact objective result while the second model obtains the result with a fuzzy number. The first model will execute rescheduling when new job comes and inserted into existing sequence or the processing time is larger than the most probable processing time and affect the objective. The second model executes rescheduling only for a new job insertion. Hence, the first model will execute more times rescheduling than the second model. The scheduling complexity of the first model is also higher than that of the second one. The second model uses a triangular fuzzy number to show the processing time and only give objective result in a range. However, the second model can reduce the rescheduling times. These two models have both advantage and disadvantage. Based on the two models, we will discuss FJSSP with new job insertion and fuzzy 
processing time constraints in the following Chapters. Extensive experiments and discussions will be shown to evaluate the two models under different scheduling heuristics and algorithms.

\subsection{Encoding and decoding methods for FJSSP}

In harmony search algorithm, one solution is called a harmony. In artificial bee colony algorithm, one solution is called a food source. To employ these two algorithms for FJSSP, this section proposed two encoding and decoding methods to present scheduling solution.

\section{MAOS encoding and decoding}

In this encoding and decoding method, a solution consists of two vectors corresponding to the machine assignment and operation scheduling sub-problems. After encoding, a solution is therefore composed of two parts:

Machine assignment vector (hereafter called MA)

Operation sequence vector (hereafter called OS)

The MA vector is to assign machine for each operation while OS is to sequence the operations. Fig. 3 - 4 (a) illustrates a machine assignment vector while Fig. 3 - 4 (b) shows the corresponding operation sequence. In MA, each element represents the machine selected for the corresponding operation. In OS, the same elements represent the different operations of the same job. For example, the first " 3 " in Fig. 3 - 4 (a) means that machine 3 is selected for operation $O_{1,1}$. The second "2" in Fig. 3 - 4 (b) represents the second operation of job 2 while the third " 4 " is the third operation of job4.

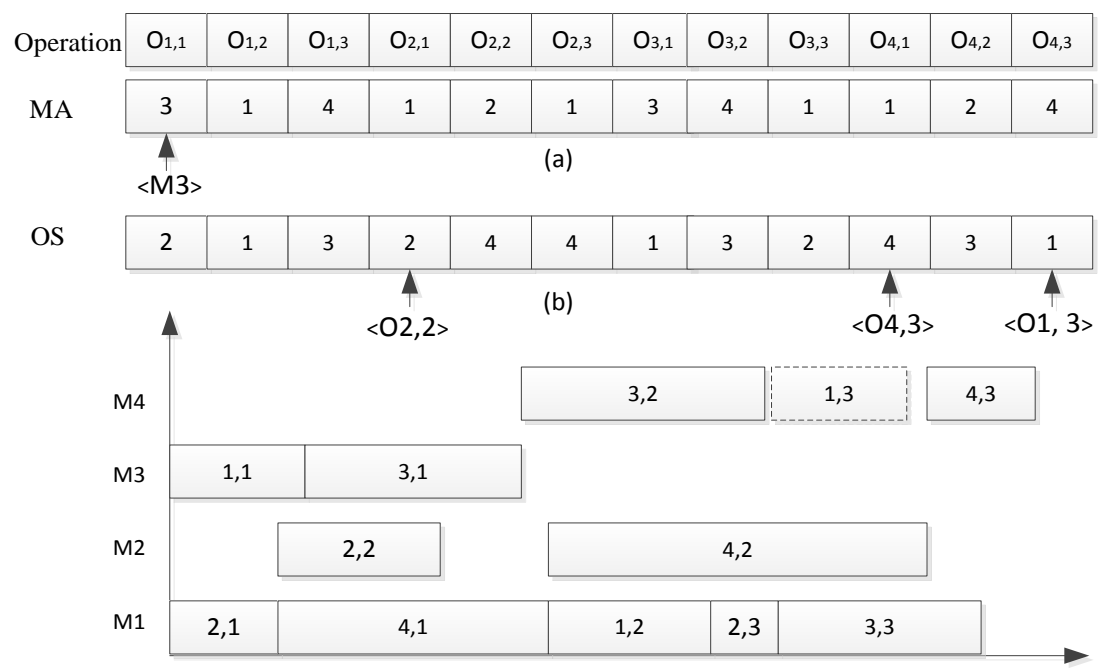

(c)

Fig. 3 - 4 Illustration of MA and OS

Pinedo [12] divided schedules into three classes: non-delay schedule, active schedule and semischedule. It has been verified in the above literature that an active schedule contains the optimal 
schedule. In an active schedule, there is no operation to be processed earlier except putting off another operation's start time or changing the order of operations. Therefore, we decode the MAOS solution to an active schedule in order to reduce the search space. The criterion for obtaining the active schedule is to find the earliest idle time interval for each operation on the corresponding machine[53]. The process of finding the earliest idle time interval is shown in Fig. 3 - 5. For example, Fig. 3 - 4 (c) is the Gantt chart decoded from the encoding shown in Fig. 3 - 4 (a) and Fig. 3 - 4 (b). It can be seen from Fig. 3 - 4 (b) that the operation $O_{4,3}$ is decoded before operation $O_{1,3}$. In Fig. 3 - 4 (a), operation $O_{1,3}$ is processed on $M_{4}$, the same machine with operation $O_{4,3}$. If the earliest idle time is not considered, the operation $O_{1,3}$ will be assigned after operation $O_{4,3}$. It can be seen from Fig. 3 - 4 (c) that the end time of $O_{3,2}$ is larger than the end time of $O_{1,2}$ and the interval between operations $O_{3,2}$ and $O_{4,3}$ is larger than the processing time of operation $O_{1,3}$. Hence, operation $O_{1,3}$ can be inserted between operations $O_{3,2}$ and $O_{4,3}$. In this way, the release time of $M_{4}$ will be the completion time of operation $O_{4,3}$. Insertion operation $O_{1,3}$ on machine $M_{4}$ does not increase the release time of machine $M_{4}$.

Procedure: Find the earliest idle time interval for operation $O_{\mathrm{i}, \mathrm{j}}$

Step1: Set I=1.

Step2: Count the interval, S, between operations I and operation I+1 on current machine.

Step3: If $\mathrm{S}$ is larger or equal the processing time of operation $O_{\mathrm{ij}}$, go to Step5, else go to Step4.

Step4: I=I+1, if I equals to the number of operations the machine has processed, go to Step6; else go to Step2.

Step5: Insert operation $O_{\mathrm{ij}}$ between operations I and $\mathrm{I}+1$ and output.

Step6: Insert $O_{\mathrm{ij}}$ after the finial operation which has been scheduled and output.

Fig. 3 - 5 The process of finding the earliest idle time

2. Three-value element encoding and decoding

In three-value element encoding decoding method, the machine assignment and operation sequencing are done and describe in an element. In each element of a solution, there are three values, job number, operation number and processing machine number. For example, a solution of 4-job, 4-machine problem is shown in Fig. 3 - 6 . The first element, $(3,1,2)$, means that the operation $O_{3,1}$ is processed on machine $M_{2}$. The element number is the total operation number of all jobs. This encoding method consists of the order of operation sequence and machine assignment.

The decoding of this method is similar to MAOS method. Table 3 - 3 shows the fuzzy processing time of each job on corresponding machines for the solution shown in Fig. 3 - 6. The solution can be decoded 
from left to right. The operations on the same machine are processed based on the order appearance in operation sequence. For example, the third element, $(4,1,4)$ and the fifth element, $(3,2,4)$ have the same processing machine $M_{4}$. The operation, $(4,1)$ will be processed first. The operation, $(3,2)$ can start when the operation $(4,1)$ is completed. In this way, the solution can be decoded to a schedule. The processing orders on all machines are $M_{1}=O_{4,2}, O_{3,3}, O_{2,3}, M_{2}=O_{3,1}, O_{2,1}, O_{1,3}, M_{3}=O_{1,1}, O_{1,2}, O_{2,2}$ and $M_{4}=O_{4,1}, O_{3,2}, O_{4,3}$. The fuzzy completion time can be computed and the fuzzy Gantt chart of this example is shown in Fig. 3 - 7.

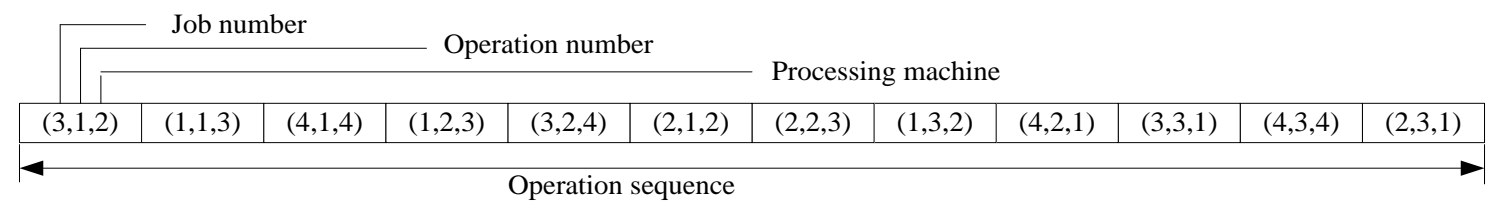

Fig. 3 - 6 A solution with three values element

Table 3 - 3 An example of fuzzy processing time for encoding and decoding

\begin{tabular}{cccccc}
\hline \multirow{2}{*}{ Job } & \multirow{2}{*}{ Operation } & \multicolumn{5}{c}{ Machine } \\
\cline { 2 - 5 } & & $M_{1}$ & $M_{2}$ & $M_{3}$ & $M_{4}$ \\
\hline \multirow{2}{*}{1} & $O_{11}$ & $(2,4,8)$ & - & $(4,5,7)$ & - \\
& $O_{12}$ & $(6,8,12)$ & $(5,8,10)$ & $(6,7,10)$ & $(4,7,9)$ \\
\multirow{2}{*}{2} & $O_{13}$ & $(8,9,12)$ & $(3,4,5)$ & - & - \\
& $O_{21}$ & & $(5,9,10)$ & & $(7,9,12)$ \\
& $O_{22}$ & $(2,4,5)$ & & $(4,7,8)$ & $(1,2,4)$ \\
3 & $O_{23}$ & $(3,5,6)$ & $(1,3,4)$ & $(1,2,5)$ & \\
& $O_{31}$ & - & $(5,6,7)$ & - & $(3,5,6))$ \\
& $O_{32}$ & $(1,2,3)$ & $(6,8,10)$ & $(5,9,11)$ & $(9,10,14)$ \\
4 & $O_{33}$ & $(1,3,4)$ & - & $(4,6,8)$ & - \\
& $O_{41}$ & - & $(5,7,9)$ & $(1,2,5)$ & $(1,2,3)$ \\
& $O_{42}$ & $(5,7,9)$ & $(4,5,7)$ & $(3,5,6)$ & $(1,2,3)$ \\
& $O_{43}$ & $(7,8,11)$ & - & - & $(1,3,4)$ \\
\hline
\end{tabular}




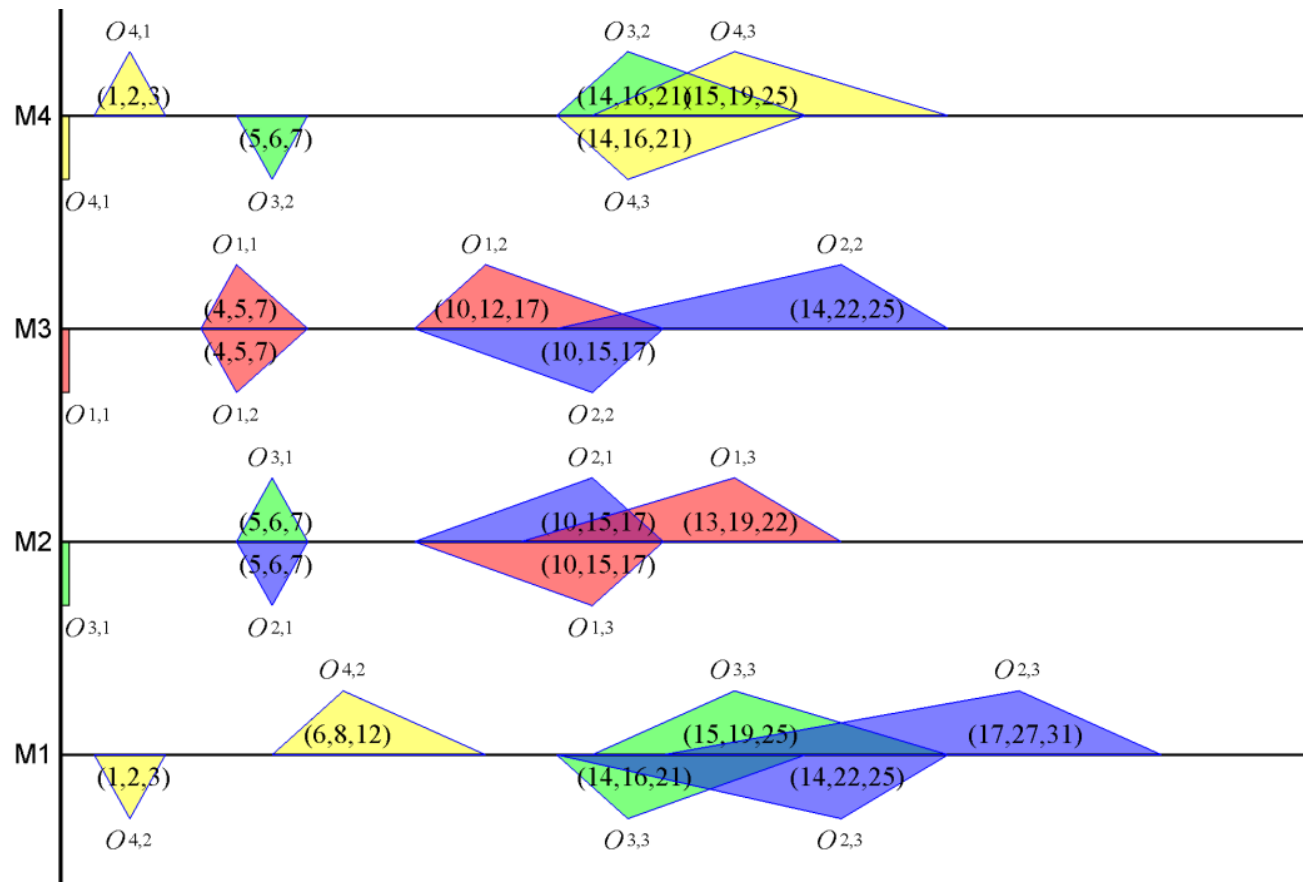

Fig. 3 - 7 Fuzzy Gantt chart of the three values element encoding and decoding 


\section{Chapter 4}

\section{DHS Algorithm for FJSSP}

\subsection{Introduction}

This Chapter proposes a Pareto-based grouping discrete harmony search algorithm (PGDHS) to solve the single objective and multi-objective flexible job shop scheduling problem (FJSSP). Two objectives, namely the maximum completion time (makespan) and the mean of earliness and tardiness, are considered simultaneously. First, two novel heuristics and several existing heuristics are employed to initialize the harmony memory. Second, multiple new harmony improvising strategies are proposed to improve the performance of harmony search algorithm. The operation sequence in a new harmony is produced based on the encoding method and the characteristics of FJSSP. Thirdly, two local search methods based on critical path and due date are embedded to enhance the exploitation capability. Finally, extensive computational experiments are carried out using well-known benchmark instances. Three widely used performance measures, number of non-dominated solutions, diversification metric and quality metric, are employed to test the performance of PGDHS algorithm. Computational results and comparisons show the efficiency and effectiveness of the proposed PGDHS algorithm for solving the multi-objective flexible job-shop scheduling problem.

The rest of this Chapter is organized as follows: In Sections 4.2-4.6, we present the PGDHS algorithm in detail. Section 4.7 presents the experiments and results compared with other algorithms in existing 
literature to demonstrate the superior performance of the proposed PGDHS algorithm. Finally, we conclude Chapter in Section 4.8.

\subsection{Initialization}

In DHS algorithm, the quality of initial HM affects the convergence speed to an optimal solution. Therefore, it is critical to generate a better quality initial HM. In this DHS algorithm, MAOS encoding and decoding method is employed to represent solution. The initialization process includes machine assignment phase and operation scheduling phase. The adoption of a mix of following rules is used to produce the machine assignment and operation scheduling for FJSSP. For example, the percentages of each initializing rule are equal.

1) Machine assignment component

Except Random rule, three heuristic rules are employed for initializing machine assignment:

$>$ Global minimum-processing time rule [16]

This rule considers both the processing time and the workload of machines and start from the operation with the global minimum processing. The processing time is added to the machine workload. For other operations, the machine with minimum processing time (workload) are fixed and assigned. The machine's workload update is also performed. This rule considers the global workload among all machines and can help find better makespan. The disadvantage is the lack of diversity.

$>$ Two-step greedy rule [29]

The operations are sorted in ascending order based on the number of selectable machines with ascending order of processing times to break ties when there is equal number of selectable machines. The machines are sorted in their workload non-decreasing order. The operation is taken from the operations list and the first machine that belongs to the machine list is assigned to the operation. The workload of this machine is updated and the machine sorting is also updated. The process iterates until all operations have been assigned to machines. The two-step greedy rule is proposed for minimization of the maximum tardiness.

Proposed minimum-completion time rule

We develop this rule based on the operation minimum processing time rule [28]. The proposed rule is as follows:

Step1: For each operation $O_{i, j}$, select machine M1 with the minimum processing time and machine M2 with the earliest feasible time from selectable machine set.

Step2: Calculate the completion time T1 and T2 of operation $\mathrm{O}_{\mathrm{i}, \mathrm{j}}$ on machines M1 and M2.

Step3: If $\mathrm{T} 1<\mathrm{T} 2, \mathrm{M} 1$ is selected for processing operation $O_{i, j}$; otherwise, $\mathrm{M} 2$ is selected for processing operation $O_{i, j}$. 
This rule considers the completion of each operation and is conducive to optimize the makespan. It can be seen from the steps of the minimum-completion time rule that the computation complexity is $O\left(2 \sum_{i=1}^{n} q_{i}\right)$

2) Operation scheduling component

Once the machine assignment is fixed, the operations on each machine should be sequenced. Except for random rule, the operation scheduling component is initialized by the following three different methods[26]:

\section{Most work remaining rule}

This method firstly orders the jobs in a descending order based on the remaining processing time. The job with the most remaining processing time will be selected first and put into the operation sequence. The iteration is repeated until all operations of all jobs have been sequenced.

$>$ Most operations remaining rule

This rule selects jobs based on the number of remaining operations. The job with the most remaining number of operations will be selected first.

$>$ Shortest processing time rule

In this approach, the operation with the shortest processing time will be selected from the next executable operations.

3) Early end time rule

The initialization methods in Sections 4.2.1 and 4.2.2 do machine assignment component first and perform the operation scheduling after machine assignment. In this section, we propose an initialization rule in the reverse process. The process is as follows:

Step1: The operation sequence is obtained by randomly shuffling the order of all operations of all jobs. Step2: For each operation $O_{i, j}$ in operation sequence, calculate the end time on all selectable machines. Step3: The machine with the minimum end time is selected for processing operation $O_{i, j}$.

\subsection{Dynamic grouping}

The small-sized HM works better than a large one for the HS algorithm and dynamic subpopulation can effectively balance the fast convergence and large diversity[138]. Hence, the DHS algorithm employs multiple small-sized dynamic sub-HMs. More specifically, the whole HM of the DHS algorithm is grouped into small-sized sub-HMs randomly in first iteration. Then, each sub-HM uses its own members to search for better solutions in the search space. After this iteration, the sub-HMs form a whole HM that is regrouped again into small-sized sub-HMs randomly, and the sub-HMs restart 
their search independently. This process, shown in Fig. 4 - 1, is continued until a termination criterion is met.

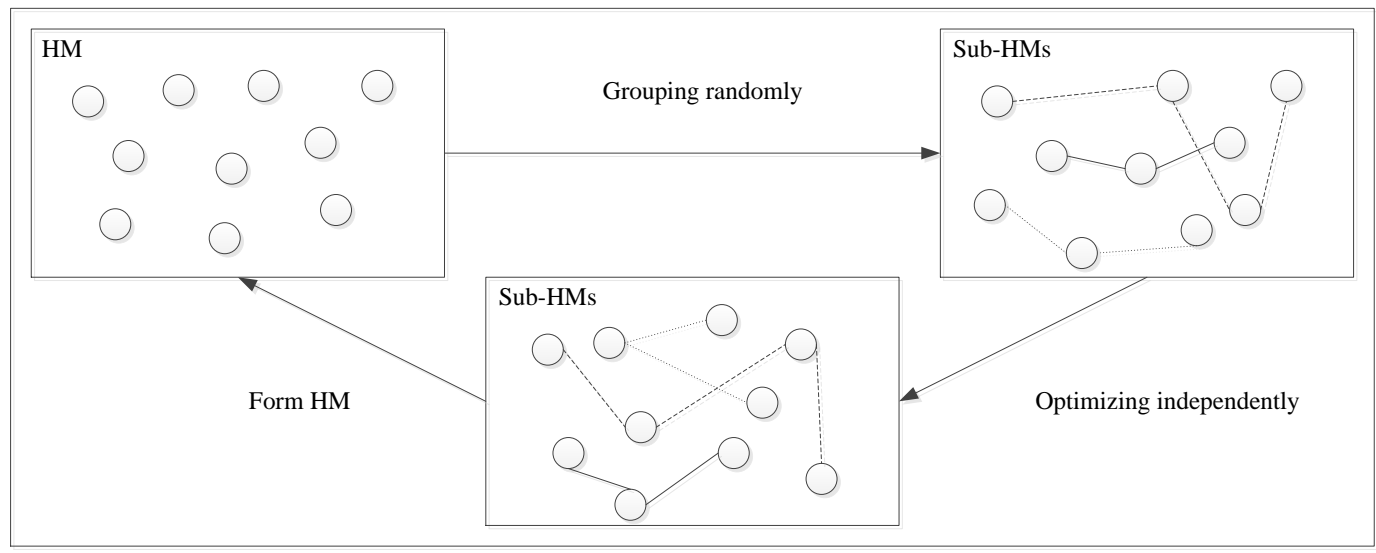

Fig. 4 - 1 Dynamic grouping optimization

\subsection{Improvising new harmony}

In DHS algorithm, each harmony includes machine assignment and operation sequence. In MA part, each element represents a machine selected for processing corresponding operation. From different harmonies, the elements at the same position are for the same operation in MA part while the elements at the same position may mean the operation of different jobs in OS part. Therefore, we improvise a new harmony for the machine assignment part and operation sequence part respectively. The process of improvising a new MA solution is shown in Fig. 4 - 2.

Procedure: Improvising the new MA part for new harmony

Step1: Generate two random numbers $R l$ and $R 2$.

Step2: If $R 1<H M C R$ and $R 2<P A R$, go to Step3; else, go to Step6;

Step3: Select one machine $k$ for current operation. If $k$ is the last machine in the set of candidate machine, go to Step4; else, go to Step5.

Step4: Select the first machine $k^{\prime}$ in the set of candidate machines for current operation.

Step5: Select the next machine $k$ ', of machine $k$ in the set of candidate machines for current operation.

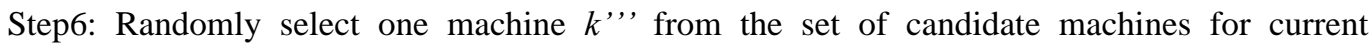
operation.

Fig. 4 - 2 Process of improvising the MA part of a new harmony

According to the characteristic of FJSSP and the encoding strategies, we use crossover operators for the OS part of a new harmony. There are several crossover operations proposed during the past decades, such as partial-mapped crossover, order crossover, cycle crossover and so on [16]. In this study, we employ a new crossover operator based on order crossover. We obtain two new harmonies from the 
current two harmonies. The employed crossover operator works for the OS part as illustrated in Fig. 4 - 3 and summarized below:

Step1: Generate a random number $R$ from 1 to number of jobs;

Step2: Copy the values from the OS part of Harmony 1 to the corresponding positions in New Harmony 1 where the values are less than or equal to $R$.

Step3: Copy the values from the OS part of Harmony 2 to the corresponding positions in New Harmony 2 where the values are larger than $R$.

Step4: From the OS part of Harmony 2, copy the values which do not appear in New Harmony 1 to the vacant positions in New Harmony 1 from left to right according to the order of the sequence in Harmony 2.

Step5: From the OS part of Harmony 1, copy the values which do not appear in New Harmony 2 to the vacant positions in New Harmony 2 from left to right according to the order of the sequence in Harmony 1.

We proposed an improvisation to generate multiple new harmonies. For each sub-HM, the number of new harmonies is the same as the number of harmonies in this sub-HM. For the OS part, there will be four operation sequences after applying crossover operation in Fig. 4 - 3. Meanwhile, we also generate four MA solutions correspondingly. We select two harmonies from the four harmonies based on domination. Two harmonies will be selected randomly if the four harmonies are non-dominated.

\begin{tabular}{|c|c|c|c|c|c|c|c|c|c|c|c|c|c|c|}
\hline Harmony 1 & 3 & 1 & 4 & 5 & 2 & 3 & 4 & 2 & 5 & 4 & 1 & 3 & 2 & 1 \\
\hline & & & & & & & & & & & & & & \\
\hline New Harmony 1 & 3 & 1 & 4 & 5 & 2 & 3 & 4 & 2 & 4 & 5 & 1 & 3 & 2 & 1 \\
\hline \multicolumn{15}{|c|}{$\mathrm{R}=3$} \\
\hline New Harmony 2 & 3 & 1 & 4 & 5 & 2 & 3 & 2 & 1 & 4 & 3 & 4 & 5 & 2 & 1 \\
\hline Harmony 2 & 2 & 1 & 4 & 5 & 3 & 2 & 2 & 1 & 4 & 3 & 4 & 5 & 3 & 1 \\
\hline
\end{tabular}

Fig. 4 - 3 Illustration of the crossover operator for OS part of a new harmony

\subsection{Local search}

In this section, we proposed two local search algorithms for FJSSP, one for makespan criterion based on critical path and another for E/T criterion based on due date of jobs.

1) Local search algorithm for makespan criterion

The critical path concept has been employed in job shop scheduling problem for improving the convergence speed[139]. In this study, we proposed a local search algorithm based on critical path for makespan criterion of FJSSP. However, we also consider the neighborhood from the different 
selectable machines of public critical operations. In the local search method, if the operation has two or more machines available for selection, we will assign a machine that is different from the current one. The first operation and the last operation in the public critical block will swap with the adjacent public critical operations.

2) Local search algorithm for $E / T$ criterion

For earliness-tardiness criterion, the jobs are divided into two groups in the proposed local search algorithm. Group one includes the jobs which are completed before the due date while group two has the jobs which are completed after the due date. For all operations on all machines, the operations which belong to the job in group one move to right adjacent position while the operations which belong to the job in group two move to left adjacent position. More specifically, the operations on the same machine are considered as pair from the first two operations. If the jobs including one pair of operations are included in different groups, the position of the pair of operations on machine will be interchanged.

\subsection{Algorithm framework}

Due to the complexity of flexible job shop scheduling problem, the proposed DHS algorithm employs multiple initialization methods, novel improvisation of new harmonies, two local search algorithms for solving the FJSSP effectively. The diversity of populations and the balance of global exploration and local exploitation are all considered. The proposed DHS algorithm is illustrated in Fig. 4 - 4. First, harmony memory and archived set are initialized. Then, harmony memory is divided into multiple Sub-HMs. Each Sub-HM executes improvising new harmonies and local search independently. Then, Sub-HMs and AS are updated. If the maximum iteration is not reached, the harmony memory is grouped again. The grouping operator and local search balance the global exploration and local exploitation. The grouping operator and improvising a new harmony stress the diversity of population during the search process. Hence, the DHS is expected to yield good performance. In each generation, the computational complexity of improvising new harmony includes two parts, Machine assignment and operations sequencing. For machine assignment, the complexity is $O\left(\sum_{i=1}^{n} q_{i}\right)$. The complexity for operation sequencing is $O\left(\log _{2} \sum_{i=1}^{n} q_{i} * \log _{2} \sum_{i=1}^{n} q_{i}\right)$. The complexity to update one harmony is $O(1)$. Hence, the computational complexity to generate new harmonies and update existing harmonies is $O\left(\left(\sum_{i=1}^{n} q_{i}+\log _{2} \sum_{i=1}^{n} q_{i} * \log _{2} \sum_{i=1}^{n} q_{i}+1\right) * H M S\right)$. 


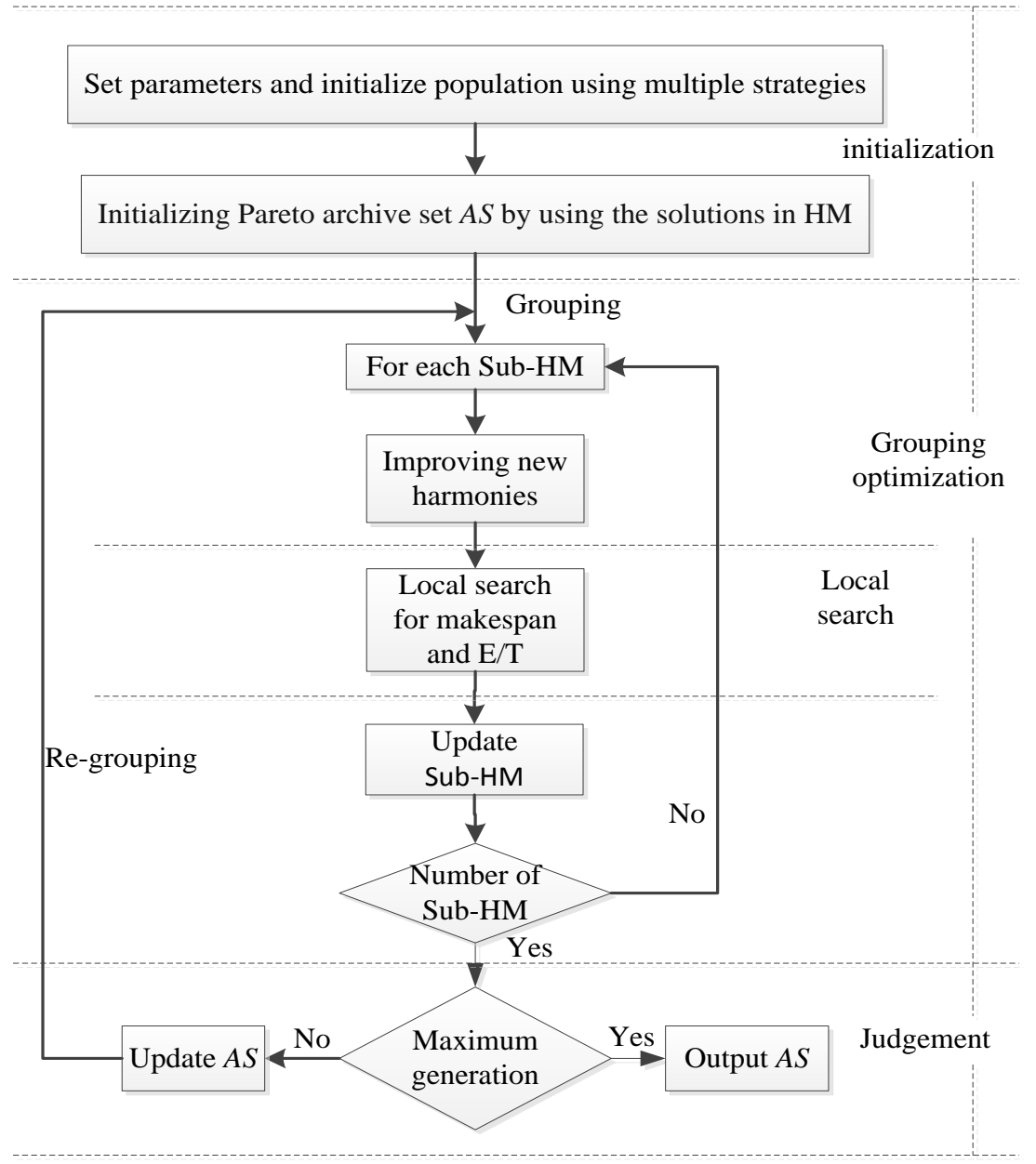

Fig. 4 - 4 The proposed DHS algorithm

\subsection{Results and comparisons}

\subsubsection{Experiment setup}

To test the performance of the proposed DHS algorithm, extensive experimental evaluation and comparisons with other methods are presented using well-known FJSSP benchmark sets. Two sets of problem instances are considered in this paper:

The first data set are five Kacem instances[140].

The second data set, called BRdata, is a set of 10 problems by Brandimare[141].

The Kacem benchmark set is composed of 5 instances with the size ranging from 4 jobs, 5 machines and 11 operations to 15 jobs, 10 machines and 60 operations. The Brandimare benchmark set includes 10 problems with the size ranging from 10 jobs, 6 machines and 55 operations to 20 jobs, 16 machines and 240 operations. In order to introduce due date in the data sets, the method inspired by Gholami[49] is employed. In this study, the due date of job $J_{i}$ can be defined by 


$$
\mathrm{D}_{i}=\left(1+\frac{T \times n}{m}\right) \times \sum_{j=1}^{n_{i}} p_{i, j}
$$

where $\mathrm{T}$ is a parameter that has been fixed as $T=0.3, n$ is the number of jobs, $m$ is the number of machines, $p_{i, j}$ is the average processing time of operation $\boldsymbol{O}_{i, j}$ on all selected machines.

Algorithms were coded in C++.net and run on Intel $2.8 \mathrm{GHz}$ PC with $1 \mathrm{~GB}$ memory. The parameters HMS, HMCR and PAR affect the performance of HS algorithm. Omran and Mahdavi [114] suggested that it was generally better to use a large value of HMCR (i.e., >=0.9). A large PAR value enhances the local exploitation ability of the algorithm while a small PAR value enlarges the search area and diversity of the HS algorithm. Based on our previous research experiences, the parameters are fixed as follows: $\mathrm{HMS}=1000, \mathrm{HMCR}=0.95$ and $\mathrm{PAR}=0.5$. Each instance is run for the 20 independent replications with $10 \times n \times m$ generation. In this paper, grouping operator is employed for improving algorithm convergence.

\subsubsection{Single objective-Makespan}

To test the performance of the proposed DHS algorithm for a single objective, we considered the makespan of the 10 instances from BRdata, which range from 10 jobs, 6 machines to 20 jobs, 15 machines. We compared the DHS with four recent algorithms, BR [141], LEGA [16], Xing [142] and HTSA[71]. The experimental results and comparisons are shown in Table 4 - 1. It can be seen from Table 4 - 1 that: 1) Our DHS algorithm achieves the optimal solutions for 7 out of 10 instances. 2) For instances MK04, MK09 and MK10, DHS algorithm outperforms the four compared algorithms. 3) For instances MK01 and MK05, DHS and HTSA obtain the same results and the results are optimal. 4) Xing, HTSA and DHS produce the optimal solution for instance MK03 while all compared algorithms can find the optimal solution for instance MK08. 5) For instances MK02 and MK06, the DHS obtains worse results than HTSA. It should be noted that the objectives of BR, LEGA, Xing and HTSA are makespan and workload while the DHS algorithm in this study considers makespan, earliness and tardiness. We also show the low bound solutions of the 10 instances. DHS algorithm can find the low bound results for MK03 and MK08. For MK01 and MK02, DHS algorithm can find results that are very near to the low bound results. For other instances, there are some distance between the DHS results and the low bound results. However, DHS algorithm has obvious advantage in computation time. For 10 instances, the computation times of DHS are from several seconds to tens of seconds that are much less than those for getting the low bound. We also count the relative error (DHS_RE) between the results by DHS and the low bound. The values of minimum, average and the maximum relative errors are $0.00,0.11$ and 0.28 , respectively. Thus, the comparisons of makespan results verify 
the efficiency of the DHS algorithm. Fig. 4 - 5 shows the Gantt chart of the best solution by DHS algorithm for instance MK01, and the value of makespan is 40 in this solution.

Table 4 - 1 The makespan results of BRdata by five algorithms

\begin{tabular}{|c|c|c|c|c|c|c|c|c|}
\hline \multirow{2}{*}{ Instance } & \multirow{2}{*}{ Size } & \multirow{2}{*}{ LB } & \multicolumn{6}{|l|}{$C M$} \\
\hline & & & BR & LEGA & Xing & HTSA & DHS & DHS_RE \\
\hline MK01 & 10,6 & 36 & 42 & $40^{a}$ & 42 & $40^{\mathrm{a}}$ & $40^{\mathrm{a}}$ & 0.11 \\
\hline MK02 & 16,6 & 24 & 32 & 29 & 28 & $26^{\mathrm{a}}$ & 28 & 0.17 \\
\hline MK03 & 15,8 & 204 & 211 & N/A & $204^{\mathrm{a}}$ & $204^{a}$ & $204^{\mathrm{a}}$ & 0.00 \\
\hline MK04 & 15,8 & 48 & 81 & 67 & 68 & 61 & $60^{a}$ & 0.25 \\
\hline MK05 & 15,4 & 168 & 186 & 176 & 177 & $172^{\mathrm{a}}$ & $172^{a}$ & 0.02 \\
\hline MK06 & 10,15 & 33 & 86 & 67 & 75 & $65^{a}$ & 67 & 0.16 \\
\hline MK07 & 20,5 & 133 & 157 & 147 & 150 & $140^{\mathrm{a}}$ & 143 & 0.08 \\
\hline MK08 & 20,10 & 523 & $523^{a}$ & $523^{a}$ & $523^{a}$ & $523^{a}$ & $523^{a}$ & 0.00 \\
\hline MK09 & 20,10 & 299 & 369 & 320 & 311 & 310 & $309^{a}$ & 0.03 \\
\hline MK10 & 20,15 & 165 & 296 & 229 & 227 & 214 & $212^{a}$ & 0.28 \\
\hline
\end{tabular}

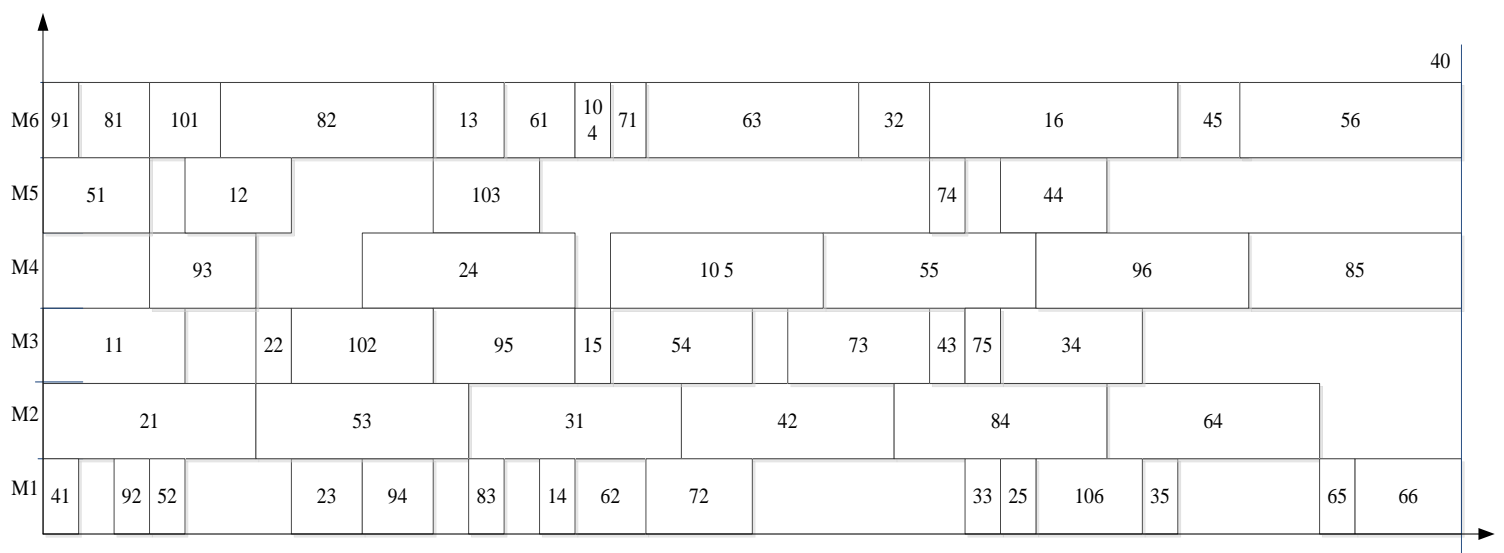

Fig. 4 - 5 Gantt chart of instance MK01 with makespan 40

\subsubsection{Pareto-based multi-objective objectives}

A multi-objective optimization problem can be described as follows:

$$
\text { Min } f(x)=\left(f_{1}(x), f_{2}(x), \cdots, f_{n}(x)\right), \quad x \in \Omega, f(x) \in R^{n}
$$

where $x$ is the decision vector in space $\Omega$ and $f(x)$ is the objective vector.

Pareto domination states that solution A dominates solution $B$ if and only if $\forall i \in\{1,2, \cdots, n\}, f_{i}(A) \leq f_{i}(B)$ and $\exists i \in\{1,2, \cdots, n\}, f_{i}(A)<f_{i}(B)$. Solution A is an optimal in the Pareto sense if there is not any solution B which dominates A. Pareto optimal set is the collection of all Pareto optimal solutions and the corresponding image in the objective space is the Pareto front. In this paper, an archive set (AS) is used to record the non-dominated solutions during the iterations. During the 
search process, if a new solution dominates one or more solutions in AS, the new solution will replace the dominated solutions. For multi-objective optimization, the algorithm should obtain more nondominated solutions with good proximity and diversity [143] with respect to the true Pareto front. In other words, it requires the algorithm to obtain more non-dominated solutions, on or closer to the optimal Pareto front, and distributed evenly over the whole Pareto front.[76]

Pareto-based multi-objective optimization algorithms are committed to find an approximate of nondominated solutions. Hence, the performance metrics are different from the single objective methods. The following widely used performance measures are used in the paper[144] with larger values representing better performances:

$>$ Number of non-dominated solutions $(\mathrm{NNdS})$

This performance measure counts the total number of non-dominated solutions generated by the compared algorithms.

$>$ Diversification metric $(\mathrm{DM})$

DM determines the diversity of non-dominated solutions by each compared algorithm. Inverted generational distance (IGD) is used by many researchers for assessing the DM performance[145]. Let $P^{*}$ be a set of uniformly distributed points in the objective space along the Pareto front $(\mathrm{PF})$. Let $P$ be the set of non-dominated solutions by compared algorithms. The inverted generational distance from $P^{*}$ to $P$ is defined as

$$
I G D(P *, P)=\frac{\sum_{v \in P^{*}} d(v, P)}{\left|P^{*}\right|}
$$

where $d(v, P)$ is the minimum Euclidean distance in the objective $V$ and the points in $P$. If $\left|P^{*}\right|$ is large enough to represent the PF well, $I G D(P *, P)$ could measure both the diversity and convergence of $P$ in a sense. To have a low value of $I G D(P *, P), P$ must be very close to the PF and should not miss any part of the whole PF. In this paper, we set $P^{*}$ as the set of Pareto solutions in PF and $P$ is the set of non-dominated solutions obtained by each algorithm.

\section{$>$ Quality metric $(\mathrm{QM})$}

In this metric, a combined overall Pareto front is constructed from all non-dominated solutions obtained by all compared algorithms. Then, the number of Pareto solutions contributed by each comparing algorithm to the overall Pareto front is counted and the percentage of the Pareto solutions belonging to each algorithm is also calculated. The algorithm with higher value is better. 
To test performance, the proposed PGDHS algorithm is compared with several recently published algorithms. These algorithms are AL+CGA presented by Kacem et al. [140], Multiagent method (Multi-Agent) proposed by $\mathrm{Wu}$ and Weng [98], the multi-objective genetic algorithm (MOGA) designed by Wang et al.[146], the particle swarm optimization and local search algorithm (MoPSO+LS) developed by Moslehi and Mahnam [64], the hybrid shuffled frog-leaping algorithm (HFSLA) proposed by Li et al. [104] and the enhanced Pareto-based artificial bee colony algorithm (EPABC) designed by Wang [136]. The two objectives are considered simultaneously, i.e. minimization of makespan (denoted by $\mathrm{C}_{\mathrm{M}}$ ) and the mean of earliness and tardiness (denoted by E/T).

Table 4 - 2 shows the results of five Kacem instances. All Pareto solutions in Table 4 - 2 are shown in boldface. It can be easily seen that: 1) Except AL+CGA, all algorithms can obtain the three Pareto solutions, $(11,7),(12,6)$ and $(16,2)$, for instance $4 \times 5.2)$ For instance $8 \times 8$, MOPSO+LS, HSFLA and EPABC identify two Pareto solutions, $(14,10)$ and $(15,8)$ while MOGA obtains three, $(14,10),(15$, 8) and (20. 5). However, PGDHS obtains one more Pareto solution, (24, 3). 3) For instance 10×7, PGDHS obtains three Pareto solutions, $(11,13),(12,9)$ and $(16,4)$. MOGA, MOPSO+LS, HSFLA and EPABC obtain just two. 4) AL+CGA algorithm obtains three of seven Pareto solutions for instance $10 \times 10$ while MOGA, MOPSO+LS, HSFLA and EPABC obtain five. PGDHS discovers all seven Pareto solutions. 5) For instance 15×10, PGDHS obtains four Pareto solutions, (11, 18), (12, 15), (18, 8), (20,6), more than other compared algorithms. Table 4 - 2 also shows the central processing unit (CPU) time of PGDHS algorithm. It also shows that proposed algorithm is efficient since the CPU time cost is low.

Table 4 - 2 Pareto-based multi-objective results of five Kacem instances

\begin{tabular}{|c|c|c|c|c|c|c|c|c|c|c|c|c|c|}
\hline \multirow{2}{*}{$\begin{array}{l}\text { Instance } \\
(\mathrm{n} \times \mathrm{m})\end{array}$} & \multicolumn{2}{|c|}{$\mathrm{AL}+\mathrm{CGA}$} & \multicolumn{2}{|c|}{ MOGA } & \multicolumn{2}{|c|}{ MOPSO+LS } & \multicolumn{2}{|c|}{ HSFLA } & \multicolumn{2}{|c|}{ EPABC } & \multicolumn{2}{|c|}{ PGDHS } & \multirow{2}{*}{$\begin{array}{r}\mathrm{CPU} \\
(\mathrm{s})\end{array}$} \\
\hline & $\mathrm{C}_{\mathrm{M}}$ & $\mathrm{E} / \mathrm{T}$ & $\mathrm{C}_{\mathrm{M}}$ & $\mathrm{E} / \mathrm{T}$ & $\mathrm{C}_{\mathrm{M}}$ & $\mathrm{E} / \mathrm{T}$ & $\mathrm{C}_{\mathrm{M}}$ & $\mathrm{E} / \mathrm{T}$ & $\mathrm{C}_{\mathrm{M}}$ & $\mathrm{E} / \mathrm{T}$ & $\mathrm{C}_{\mathrm{M}}$ & $\mathrm{E} / \mathrm{T}$ & \\
\hline \multirow[t]{3}{*}{$4 \times 5$} & 16 & 6 & 11 & 7 & 11 & 7 & 11 & 7 & 11 & 7 & 11 & 7 & 0.32 \\
\hline & 18 & 4 & 12 & 6 & 12 & 6 & 12 & 6 & 12 & 6 & 12 & 6 & \\
\hline & & & 16 & 2 & 16 & 2 & 16 & 2 & 16 & 2 & 16 & 2 & \\
\hline \multirow[t]{4}{*}{$8 \times 8$} & 15 & 11 & 14 & 10 & 14 & 10 & 14 & 10 & 14 & 10 & 14 & 10 & 2.12 \\
\hline & 24 & 8 & 15 & 8 & 15 & 8 & 15 & 8 & 15 & 8 & 15 & 8 & \\
\hline & & & 20 & 5 & & & & & & & 20 & 5 & \\
\hline & & & & & & & & & & & 24 & 3 & \\
\hline \multirow[t]{3}{*}{$10 \times 7$} & 12 & 13 & 11 & 13 & 11 & 13 & 11 & 13 & 11 & 13 & 11 & 13 & 2.50 \\
\hline & 13 & 9 & 12 & 9 & 12 & 9 & 12 & 9 & 12 & 9 & 12 & 9 & \\
\hline & & & 17 & 6 & 17 & 6 & 17 & 6 & 17 & 6 & 16 & 4 & \\
\hline \multirow[t]{2}{*}{$10 \times 10$} & 7 & 13 & 7 & 12 & 7 & 12 & 7 & 12 & 7 & 12 & 7 & 12 & 3.49 \\
\hline & 8 & 11 & 8 & 11 & 8 & 11 & 8 & 11 & 8 & 11 & 8 & 11 & \\
\hline
\end{tabular}




\begin{tabular}{cccccccccccccc}
\hline & 10 & 7 & 10 & 7 & 10 & 7 & 10 & 7 & 10 & 7 & 10 & 7 & \\
& 11 & 6 & 11 & 6 & 11 & 6 & 11 & 6 & 11 & 6 & 11 & 6 & \\
& & & 12 & 5 & 12 & 5 & 12 & 5 & 12 & 5 & 12 & 5 & \\
& & & & & & & & & & & 15 & 4 & \\
& & & & & & & & & & & 16 & 3 & \\
& & & & & & & & & & & 16 & \\
& 25 & 15 & 11 & 18 & 11 & 18 & 11 & 18 & 11 & 18 & 11 & 18 & 9.06 \\
& & 12 & 15 & 12 & 16 & 12 & 15 & 12 & 15 & 12 & 15 & \\
& & 18 & 8 & 18 & 11 & 18 & 10 & 18 & 10 & 18 & 8 & \\
& & & & & & 20 & 9 & 20 & 9 & 20 & 6 & \\
\hline
\end{tabular}

Table 4 - 3 shows the number of non-dominated solutions and quality metric of all compared algorithms for five Kacem instances. The second column shows the number of Pareto solutions in PF. For each compared algorithm, the total number of non-dominated solutions, the number of nondominated solutions which belong to the overall combined PF and the average quality metric values for five instances are also calculated. For example, AL+CGA can find two non-dominated solutions for instance 5, but both of them are not in the overall combined PF. For the same instance, MOGA obtained three non-dominated solutions and all of them are in PF while PGDHS algorithm can find four non-dominated solutions and all of them are in the overall combined PF. It can be seen that the proposed PGDHS algorithm has 21 non-dominated solutions and all these non-dominated solutions are in the overall combined PF. The quality metric values obtained by PGDHS are all $100 \%$ for five instances. It means that PGDHS can obtain all Pareto solutions for five instances. For above mentioned performance measures, PGDHS has better results than the compared five algorithms.

In addition, the diversification metric values of each compared algorithms are computed for five instances. The minimum and standard deviation of IGD-metric values are shown in Table 4 - 4. It can be seen from Table 4 - 4 that five algorithms can obtain the best minimum values for five instances except AL+CGA. Among six compared algorithms, only PGDHS found the best mean and standard deviation values for five instances. Hence, the diversification metric of PGDHS algorithm is better than the five compared algorithms.

Hence, the PGDHS is better than compared five algorithms for five Kacem instances. As an example, Fig. 4 - 6 shows the Gantt chart of Pareto solution $(24,3)$ of Kacem instance $8 \times 8$ in which the due dates of all jobs are 22, 31, 22, 23, 33, 28, 26 and 31.

Table 4 - 3 Number of Non-dominate solutions (NNdS) and quality metric (QM) for 5 Kacem instances

\begin{tabular}{|c|c|c|c|c|c|c|c|c|c|c|c|c|c|}
\hline \multirow{2}{*}{$\begin{array}{l}\text { Instance } \\
(\mathrm{n} \times \mathrm{m})\end{array}$} & \multirow[b]{2}{*}{$\mathrm{PF}$} & \multicolumn{2}{|c|}{$\mathrm{AL}+\mathrm{CGA}$} & \multicolumn{2}{|l|}{ MOGA } & \multicolumn{2}{|c|}{ MOPSO+LS } & \multicolumn{2}{|l|}{ HSFLA } & \multicolumn{2}{|l|}{ EPABC } & \multicolumn{2}{|l|}{ PGDHS } \\
\hline & & NS/PF & QM\% & NS/PF & QM\% & NS/PF & QM\% & NS/PF & QM\% & $\mathrm{NS} / \mathrm{PF}$ & QM\% & NS/PF & QM\% \\
\hline
\end{tabular}




\begin{tabular}{llllllllllllll}
\hline $4 \times 5$ & 3 & $2 / 0$ & 0.0 & $3 / 3$ & 100.0 & $3 / 3$ & 100.0 & $3 / 3$ & 100.0 & $3 / 3$ & 100.0 & $3 / 3$ & 100 \\
$8 \times 8$ & 4 & $2 / 0$ & 0.0 & $3 / 3$ & 75.0 & $2 / 2$ & 50.0 & $2 / 2$ & 50.0 & $2 / 2$ & 50.0 & $4 / 4$ & 100 \\
$10 \times 7$ & 3 & $2 / 0$ & 0.0 & $3 / 2$ & 66.7 & $3 / 2$ & 66.7 & $3 / 2$ & 66.7 & $3 / 2$ & 66.7 & $3 / 3$ & 100 \\
$10 \times 10$ & 7 & $4 / 3$ & 42.9 & $5 / 5$ & 71.4 & $5 / 5$ & 71.4 & $5 / 5$ & 71.4 & $5 / 5$ & 71.4 & $7 / 7$ & 100 \\
$15 \times 10$ & 4 & $2 / 0$ & 0.0 & $3 / 3$ & 75.0 & $3 / 1$ & 25.0 & $4 / 2$ & 50.0 & $4 / 2$ & 50.0 & $4 / 4$ & 100 \\
Sum & 21 & 12 & 8.6 & 17 & 77.6 & 16 & 62.6 & 17 & 67.6 & 17 & 67.6 & 21 & 100 \\
Ave & 3 & & 16 & & 13 & & 14 & & 14 & & 21 & \\
\hline
\end{tabular}

Table 4 - 4 Diversification metric (DM) for 5 Kacem instances

\begin{tabular}{lccccccccccccc}
\hline $\begin{array}{l}\text { Instance } \\
(\mathrm{n} \times \mathrm{m})\end{array}$ & \multicolumn{2}{c}{ AL+CGA } & \multicolumn{2}{c}{ MOGA } & \multicolumn{3}{c}{ MOPSO+LS } & \multicolumn{2}{c}{ HSFLA } & \multicolumn{2}{c}{ EPABC } & \multicolumn{3}{c}{ PGDHS } \\
$\mathrm{min}$ & $\mathrm{sd}$ & $\mathrm{min}$ & $\mathrm{sd}$ & $\mathrm{min}$ & $\mathrm{sd}$ & $\mathrm{min}$ & $\mathrm{sd}$ & $\mathrm{min}$ & $\mathrm{sd}$ & $\mathrm{min}$ & $\mathrm{mean}$ & $\mathrm{sd}$ \\
\hline $4 \times 5$ & 0.94 & 0.38 & 0.00 & 0.00 & 0.00 & 0.00 & 0.00 & 0.00 & 0.00 & 0.00 & 0.00 & 0.00 & 0.00 \\
$8 \times 8$ & 0.35 & 0.43 & 0.00 & 0.56 & 0.00 & 1.25 & 0.00 & 1.25 & 0.00 & 1.25 & 0.00 & 0.00 & 0.00 \\
$10 \times 7$ & 0.33 & 0.93 & 0.00 & 0.43 & 0.00 & 0.43 & 0.00 & 0.43 & 0.00 & 0.43 & 0.00 & 0.00 & 0.00 \\
$10 \times 10$ & 0.00 & 0.34 & 0.00 & 0.27 & 0.00 & 0.27 & 0.00 & 0.27 & 0.00 & 0.27 & 0.00 & 0.00 & 0.00 \\
$15 \times 10$ & 1.60 & 0.72 & 0.00 & 0.35 & 0.00 & 0.59 & 0.00 & 0.38 & 0.00 & 0.38 & 0.00 & 0.00 & 0.00 \\
\hline
\end{tabular}

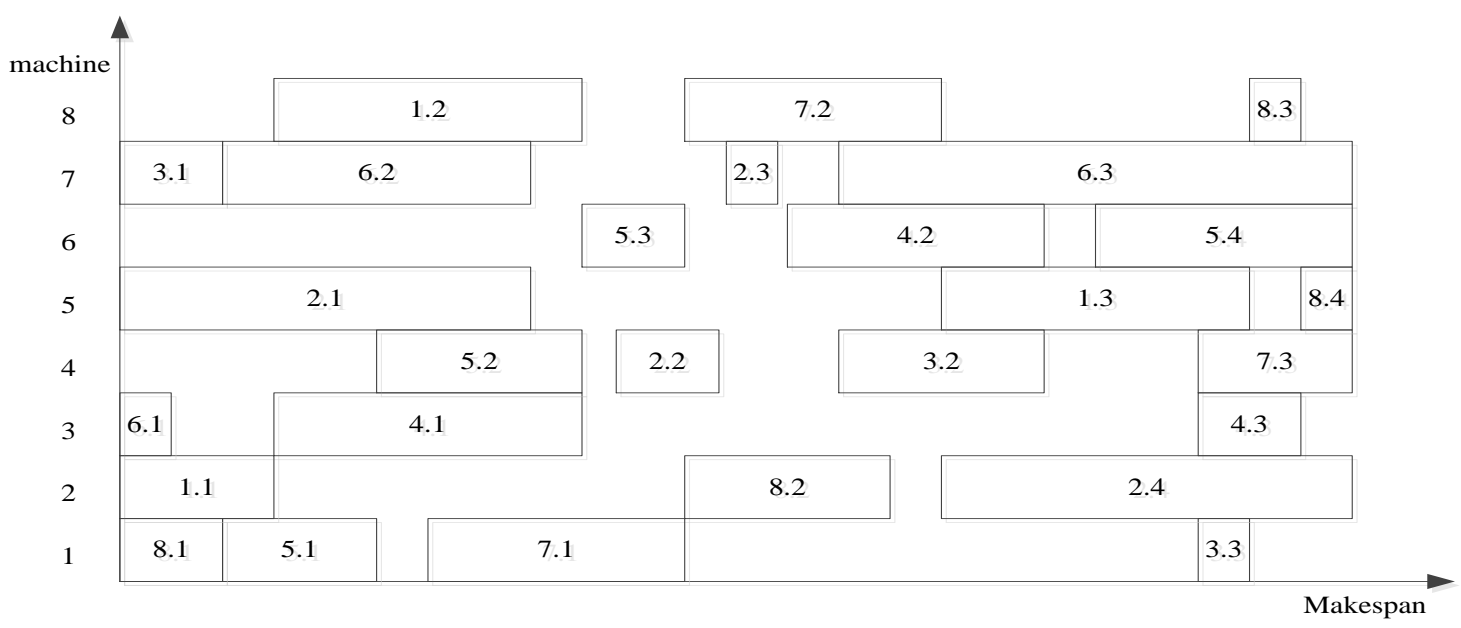

Fig. 4 - 6 Gantt chart of the solution $(24,3)$ for Kacem instance $8 \times 8$

PGDHS algorithm is compared with four very recent algorithms, i.e. Multi-Agent [98], MOGA [144], HSFLA [104] and EPABC [136] using BRdata set. The results obtained by compared algorithms are given in Table 4 - 5. All the Pareto solutions in Table 4 - 5 are also in boldface.

As shown in Table 4 - 5, the proposed PGDHS algorithm is better than or competitive to four compared algorithms. 1) For instance MK01, all five algorithms can locate Pareto solution $(42,3)$. Except Multi-Agent algorithm, other four algorithms can also obtain Pareto solution (40, 4). For instance MK 03, all compared algorithms obtain Pareto solution (204, 21). But, only PGDHS algorithm obtains Pareto solutions $(220,20)$ and $(225,18)$. 2) Multi-Agent can locate only one non- 
dominated solution $(42,3)$ for instance MK 01 and $(204,21)$ for instance MK03. 3) MOGA, HSFLA and EPABC obtain two Pareto solutions, $(26,5)$ and $(27,3)$ for instance MK 02 and $(173,59)$ and $(174,58)$ for instance MK 05. However, PGDHS obtains one more Pareto solution $(31,2)$ for instance MK 02 and obtains four more Pareto solutions $(178,41),(180,38),(181,37)$ and $(190,36)$ for instance MK 05. 4) MOGA discovers a Pareto solution $(60,19)$ for instance MK 04 and a Pareto solution (139, 26) for instance MK 07. These two Pareto solutions are not found by PGDHS and other compared algorithms. However, PGDHS obtains four Pareto solutions for instance MK 04 and three of them are not found by MOGA. At the same time, PGDHS discovers five Pareto solutions for instance MK 07 and two of them are not found by MOGA. 5) PGDHS obtains one Pareto solution $(62,2)$ for instance MK 06. Meanwhile, PGDHS identifies two Pareto solutions $(523,171)$ and $(525,165)$ for instance MK 08. But, other compared algorithms could not find any non-dominated solution for these two instances. 6) For instance MK 09 and MK 10, PGDHS obtains five and four Pareto solutions respectively while other compared algorithms could not find any Pareto solutions for these two instances. Table 4 - 5 also shows the CPU times for each instance.

Table 4 - 6 shows the number of non-dominated solutions and quality metric values of all compared algorithm for 10 BRdata instances. For each compared algorithm, the number of Pareto solutions, the total number of non-dominated solutions, the number of non-dominated solutions which belong to the overall combined PF and the average quality metric values are also calculated. It can be seen from Table 4 - 6 that the proposed PGDHS algorithm has 35 non-dominated solutions. This total count is more than the number of solutions obtained by other compared algorithms (Multi-Agent 2, MOGA 13, HSFLA 10 and EPABC 10). The PGDHS algorithm also obtained maximum Pareto solutions compared to other algorithms. For 10 instances, PGDHS algorithm found 35 out of 38 Pareto solutions in the overall combined PF. The quality metric values obtained by PGDHS are 100 for seven out of ten instances. The corresponding values for MK04, MK05 and MK07 are 80.00, 85.71 and 83.33, respectively. The average quality metric value of PGDHS is the best one among the compared algorithms.

Table 4 - 7 shows the diversification metric values of each compared algorithms for 10 BRdata instances. The mean and standard deviation of IGD diversification metric values of each algorithm are also calculated. For 10 instances, the minimum values of PGDHS are all zero. At the same time, the mean and standard deviation values of PGDHS are also zero for 7 instances except MK04, MK05 and MK07 instances. For instances MK05 and MK07, the mean and standard deviation values are (0.06, $0.15)$ and $(0.09,0.22)$, respectively. These results are better than the four compared algorithms even though the values are larger than zero. For instance MK04, PGDHS can also obtain the best mean value, 0.18 , while the standard deviation value, 0.40 , is worse than four compared algorithms. MOGA, 
HSFLA and EPABC can also obtain the best mean and standard deviation values for instance MK01. However, PGDHS algorithm has the best competitive values for mean and standard deviation of 10 instances because most results generated by PGDHS are better than those by the four compared algorithms are.

Once again, it verifies that the PGDHS possesses superior performance than the four compared algorithms in terms of several widely used performance measures. As an example, Fig. 4 - 7 shows the Gantt chart of Pareto solution $(40,4)$ of MK 01 instance $10 \times 6$ in which the due dates of job1-10 are $30,28,31,25,42,33,19,36,33$ and 30 .

Table 4 - 5 Pareto-based multi-objective results for BRdata

\begin{tabular}{|c|c|c|c|c|c|c|c|c|c|c|c|}
\hline \multirow{2}{*}{ Instance } & \multicolumn{2}{|c|}{ Multi-Agent } & \multicolumn{2}{|c|}{ MOGA } & \multicolumn{2}{|c|}{ HSFLA } & \multicolumn{2}{|c|}{ EPABC } & \multicolumn{2}{|c|}{ PGDHS } & \multirow{2}{*}{$\begin{array}{l}\text { CPU } \\
\text { (s) }\end{array}$} \\
\hline & $\mathrm{C}_{\mathrm{M}}$ & $\mathrm{E} / \mathrm{T}$ & $\mathrm{C}_{\mathrm{M}}$ & $\mathrm{E} / \mathrm{T}$ & $\mathrm{C}_{\mathrm{M}}$ & $\mathrm{E} / \mathrm{T}$ & $\mathrm{C}_{\mathrm{M}}$ & $\mathrm{E} / \mathrm{T}$ & $\mathrm{C}_{\mathrm{M}}$ & $\mathrm{E} / \mathrm{T}$ & \\
\hline \multirow[t]{2}{*}{ MK01 } & & & 40 & 4 & 40 & 4 & 40 & 4 & 40 & 4 & 5.2 \\
\hline & 42 & 3 & 42 & 3 & 42 & 3 & 42 & 3 & 42 & 3 & \\
\hline \multirow[t]{3}{*}{ MK02 } & 28 & 3 & 26 & 5 & 26 & 5 & 26 & 5 & 26 & 5 & 5.3 \\
\hline & & & 27 & 3 & 27 & 3 & 27 & 3 & 27 & 3 & \\
\hline & & & & & & & & & 31 & 2 & \\
\hline \multirow[t]{3}{*}{ MK03 } & 204 & 21 & 204 & 21 & 204 & 21 & 204 & 21 & 204 & 21 & 24.1 \\
\hline & & & & & & & & & 220 & 20 & \\
\hline & & & & & & & & & 225 & 18 & \\
\hline \multirow[t]{4}{*}{ MK04 } & 67 & 17 & 60 & 19 & 62 & 15 & 62 & 15 & 62 & 15 & 14.7 \\
\hline & 70 & 16 & 62 & 15 & 68 & 12 & 68 & 12 & 65 & 12 & \\
\hline & & & 68 & 12 & 71 & 11 & 73 & 11 & 68 & 10 & \\
\hline & & & & & & & & & 69 & 8 & \\
\hline \multirow[t]{6}{*}{ MK05 } & 174 & 61 & 173 & 59 & 173 & 59 & 173 & 59 & 173 & 59 & 9.0 \\
\hline & 178 & 58 & 174 & 58 & 174 & 58 & 174 & 58 & 174 & 58 & \\
\hline & & & 176 & 56 & 176 & 56 & 176 & 56 & 178 & 41 & \\
\hline & & & 184 & 49 & 182 & 52 & 179 & 48 & 180 & 38 & \\
\hline & & & & & & & & & 181 & 37 & \\
\hline & & & & & & & & & 190 & 36 & \\
\hline \multirow[t]{3}{*}{ MK06 } & 67 & 7 & 62 & 9 & 64 & 5 & 64 & 5 & 62 & 4 & 25.7 \\
\hline & 77 & 6 & 64 & 5 & 66 & 4 & 66 & 4 & & & \\
\hline & & & 66 & 4 & & & & & & & \\
\hline \multirow[t]{5}{*}{ MK07 } & 144 & 23 & 139 & 26 & 141 & 21 & 141 & 21 & 140 & 23 & 13.9 \\
\hline & 146 & 20 & 140 & 23 & 144 & 20 & 144 & 20 & 141 & 21 & \\
\hline & & & 141 & 21 & & & & & 144 & 20 & \\
\hline & & & 144 & 20 & & & & & 150 & 16 & \\
\hline & & & & & & & & & 152 & 14 & \\
\hline \multirow[t]{3}{*}{ MK08 } & 523 & 177 & 523 & 177 & 523 & 177 & 523 & 177 & 523 & 171 & 53.6 \\
\hline & 525 & 171 & 525 & 171 & 525 & 171 & 525 & 171 & 525 & 165 & \\
\hline & 526 & 170 & 526 & 170 & 526 & 170 & 526 & 170 & & & \\
\hline
\end{tabular}




\begin{tabular}{|c|c|c|c|c|c|c|c|c|c|c|c|}
\hline & 527 & 169 & 527 & 169 & 527 & 169 & 527 & 169 & & & \\
\hline \multirow[t]{5}{*}{ MK09 } & 311 & 110 & 310 & 112 & 311 & 110 & 309 & 114 & 307 & 96 & 62.4 \\
\hline & & & 311 & 110 & & & 310 & 112 & 316 & 80 & \\
\hline & & & & & & & 311 & 110 & 320 & 77 & \\
\hline & & & & & & & & & 333 & 68 & \\
\hline & & & & & & & & & 336 & 65 & \\
\hline \multirow[t]{4}{*}{ MK10 } & 215 & 51 & 214 & 53 & 215 & 51 & 215 & 48 & 211 & 48 & 78.9 \\
\hline & 217 & 45 & 216 & 45 & 217 & 45 & 217 & 45 & 212 & 40 & \\
\hline & 218 & 41 & 218 & 41 & 218 & 41 & 218 & 41 & 213 & 36 & \\
\hline & & & & & & & & & 215 & 31 & \\
\hline
\end{tabular}

Table 4 - 6 Number Non-dominate solutions (NNdS) and quality metric (QM) for BRdata

\begin{tabular}{llllllllllll}
\hline Instance & \multirow{2}{*}{ PF } & \multicolumn{2}{c}{ Multi-Agent } & \multicolumn{2}{c}{ MOGA } & \multicolumn{2}{c}{ HSFLA } & \multicolumn{2}{c}{ EPABC } & \multicolumn{2}{c}{ PGDHS } \\
\cline { 3 - 11 } & & NS/PF & QM\% & NS/PF & QM\% & NS/PF & QM\% & NS/PF & QM\% & NS/PF & QM\% \\
\hline MK01 & 2 & $1 / 1$ & 50.00 & $2 / 2$ & 100.00 & $2 / 2$ & 100.00 & $2 / 2$ & 100.00 & $2 / 2$ & 100.00 \\
MK02 & 3 & $1 / 0$ & 0.00 & $2 / 2$ & 66.7 & $2 / 2$ & 66.7 & $2 / 2$ & 66.7 & $3 / 3$ & 100.00 \\
MK03 & 3 & $1 / 1$ & 33.33 & $1 / 1$ & 33.33 & $1 / 1$ & 33.33 & $1 / 1$ & 33.33 & $3 / 3$ & 100.00 \\
MK04 & 5 & $2 / 0$ & 0.00 & $3 / 2$ & 40.00 & $3 / 1$ & 20.00 & $3 / 1$ & 20.00 & $4 / 4$ & 80.00 \\
MK05 & 7 & $2 / 0$ & 0.00 & $4 / 3$ & 42.86 & $4 / 3$ & 42.86 & $4 / 3$ & 42.86 & $6 / 6$ & 85.71 \\
MK06 & 1 & $2 / 0$ & 0.00 & $3 / 0$ & 0.00 & $2 / 0$ & 0.00 & $2 / 0$ & 0.00 & $1 / 1$ & 100.00 \\
MK07 & 6 & $2 / 0$ & 0.00 & $4 / 4$ & 66.67 & $2 / 2$ & 33.33 & $2 / 2$ & 33.33 & $5 / 5$ & 83.33 \\
MK08 & 2 & $4 / 0$ & 0.00 & $4 / 0$ & 0.00 & $4 / 0$ & 0.00 & $4 / 0$ & 0.00 & $2 / 2$ & 100.00 \\
MK09 & 5 & $1 / 0$ & 0.00 & $2 / 0$ & 0.00 & $1 / 0$ & 0.00 & $3 / 0$ & 0.00 & $5 / 5$ & 100.00 \\
MK10 & 4 & $3 / 0$ & 0.00 & $3 / 0$ & 0.00 & $3 / 0$ & 0.00 & $3 / 0$ & 0.00 & $4 / 4$ & 100.00 \\
Sum/Ave & 38 & $19 / 2$ & 8.33 & $28 / 14$ & 34.96 & $24 / 11$ & 29.62 & $26 / 11$ & 29.62 & $35 / 35$ & 94.90 \\
\hline
\end{tabular}

Table 4 - 7 Diversification metric (DM) for 10 BRdata instances

\begin{tabular}{lllllllllllll}
\hline \multirow{2}{*}{ Instance } & \multicolumn{2}{c}{ Multi-Agent } & \multicolumn{2}{c}{ MOGA } & \multicolumn{2}{c}{ HSFLA } & \multicolumn{2}{c}{ EPABC } & \multicolumn{3}{c}{ PGDHS } \\
& mean & sd & mean & sd & mean & sd & mean & sd & min & mean & sd \\
\hline MK01 & 0.56 & 0.79 & 0.00 & 0.00 & 0.00 & 0.00 & 0.00 & 0.00 & 0.00 & 0.00 & 0.00 \\
MK02 & 0.78 & 0.39 & 0.46 & 0.79 & 0.46 & 0.79 & 0.46 & 0.79 & 0.00 & 0.00 & 0.00 \\
MK03 & 4.14 & 3.69 & 4.14 & 3.69 & 4.14 & 3.69 & 4.14 & 3.69 & 0.00 & 0.00 & 0.00 \\
MK04 & 1.30 & 0.24 & 0.36 & 0.37 & 0.52 & 0.34 & 0.54 & 0.36 & 0.00 & 0.18 & 0.40 \\
MK05 & 1.87 & 1.43 & 0.99 & 0.94 & 1.20 & 1.15 & 0.91 & 0.94 & 0.00 & 0.06 & 0.15 \\
MK06 & 5.83 & 0.00 & 2.24 & 0.00 & 2.24 & 0.00 & 2.24 & 0.00 & 0.00 & 0.00 & 0.00 \\
MK07 & 0.82 & 0.37 & 0.48 & 0.76 & 0.69 & 0.68 & 0.69 & 0.68 & 0.00 & 0.09 & 0.22 \\
MK08 & 1.62 & 0.87 & 1.62 & 0.87 & 1.62 & 0.87 & 1.62 & 0.87 & 0.00 & 0.00 & 0.00 \\
MK09 & 7.12 & 2.94 & 7.12 & 2.94 & 7.12 & 2.94 & 7.12 & 2.94 & 0.00 & 0.00 & 0.00 \\
MK10 & 1.79 & 0.59 & 1.84 & 0.53 & 1.79 & 0.59 & 1.73 & 0.67 & 0.00 & 0.00 & 0.00 \\
\hline
\end{tabular}




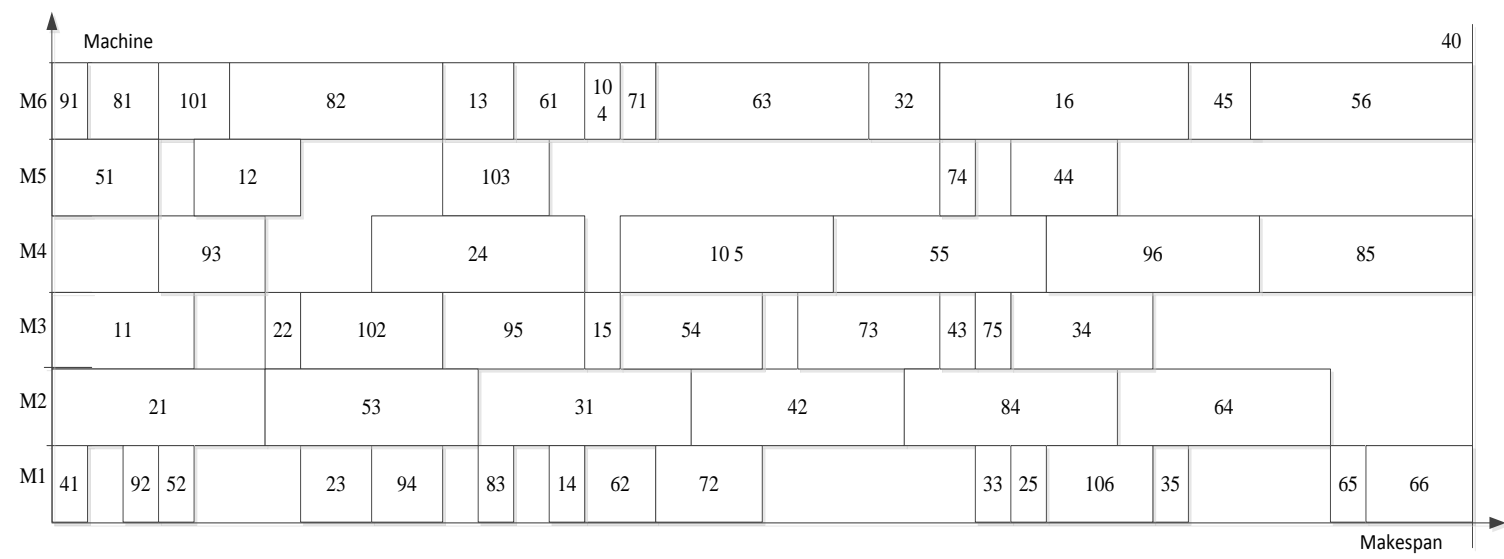

Fig. 4 - 7 Gantt chart of solution $(40,4)$ for MK01 instance $10 \times 6$

\subsection{Conclusions}

This Chapter proposed a grouping discrete harmony search algorithm for flexible job shop scheduling with makespan, and earliness-tardiness criteria. Two heuristics were developed for initializing the harmony memory. Sub-Harmony memory was employed to improve the diversity of harmony for a large population in harmony memory. Based on the problem characteristics and solution representation, a method was developed to improvise new harmonies. In order to improve the search ability and reduce the search space, two local search algorithms were proposed based on critical path and due date for makespan and E/T criterion, respectively. The large population and local search algorithms balanced the capability of exploration and exploitation. It has been demonstrated by simulation results based on the widely studied benchmarks and comparisons with the state-of-the-art algorithms that the proposed grouping discrete harmony search algorithm is better than the existing algorithms in terms of quality and the number of obtained overall non-dominated solutions. 


\section{Chapter 5}

\section{Two-stage ABC Algorithm for FJSSP with New Job Insertion}

\subsection{Introduction}

This Chapter addresses flexible job-shop scheduling problem (FJSSP) with new job insertion. The new job insertion is one of seven important characteristics in remanufacturing. Scheduling problem in remanufacturing can be divided into two stages: scheduling and re-scheduling when a new job arrives. A two-stage artificial bee colony (TABC) algorithm is proposed for scheduling and re-scheduling with new job(s) insertion. The objective is to minimize maximum complete time (makespan). An ensemble local search is proposed to improve the convergence of TABC. Three re-scheduling strategies are proposed for new job insertion. Extensive computational experiments are carried out using fifteen well-known benchmark instances with eight instances from remanufacturing. Three re-scheduling strategies are compared and discussed. For scheduling performance, TABC is compared to several existing algorithms. For re-scheduling performance, TABC is compared to several simple heuristics and proposed hybrid heuristics. The results and comparisons show that the TABC algorithm is 
effectiveness and efficiency for solving FJSSP with new job insertion. In the TABC algorithm, MAOS encoding and decoding method is employed to represent a solution.

The remainder of this Chapter is organized as follows. Section 5.2 describes the three rescheduling strategies. In Section 5.3, an ensemble local search algorithm is proposed. Section 5.4 presents the two-stage ABC algorithm framework. Experimental design, comparison and discussion are shown in Sections 5.5-5.7. We conclude this Chapter in Section 5.8.

\subsection{Rescheduling strategies}

In this section, we proposed three rescheduling strategies for the new job insertion. The three different rescheduling strategies are shown as follows:

1. Strategy $\mathrm{I}$ is just reschedule the new inserted job(s). Existing scheduling scheme remains. In rescheduling strategy $\mathrm{I}$, the machines are available when all the assigned operations are completed. It means that machines may have different start time when rescheduling is implemented. Hence, machine start time must be considered for rescheduling in strategy I. The rescheduling problem becomes FJSSP with different machine starting times.

2. Strategy II is rescheduling both new insertion job(s) and the existing jobs' operations that are not started at the new job insertion time. The scheduling solution for existing jobs' operations may be changed. In strategy II, some machines may be performing some operations of existing jobs at the new job insertion time. The machines are available when the processing operations are completed. The existing jobs are also available for rescheduling when the corresponding operations are completed. It means that both machines and jobs have different start times when rescheduling is implemented. Hence, both machine start times and job start times must be considered for rescheduling in strategy II. The rescheduling problem becomes FJSSP with different machine starting times and different job starting times.

3. Strategy III is free-time-gap rescheduling based on existing scheduling scheme. Similar to Strategy I, the existing scheduling schema is remained. The new job(s) is (are) scheduled on all gaps of all machines after arrival time. It means that one machine is available if it has free-timegap on the existing scheduling schema. In this condition, the machine can be employed for scheduling new job(s). After considered all free gaps on all machine, the same scheduling strategy in Strategy I is employed for scheduling remains operations. Compared to Strategy I, Strategy III has higher time complexity.

\subsection{Ensemble local search}


Critical path theory is employed for solving the FJSSP by many researchers. In one solution, there may be one or more critical paths. Each operation on one critical path is called a critical operation. This Chapter proposed an ensemble local search on a machine. The machines are sorted based on the number of critical operations processed on them. The first ensemble local search is executed on the machine with the maximum critical operations. There are five local search operators, one Insert, one Swap, two Inserts, two Swaps, one Insert and one Swap. These five operators are stored in an operator pool. In the beginning, each operator has the same ratio to be selected for generating a neighbor solution. The operator improving the solution will be remained in operator pool while the operator not improving the solution will be deleted from the operator pool. The ensemble local search will be terminated when all the operators have been deleted from operator pool. The next machine will be executed ensemble local search until the maximum local search iterations are met. The detail steps of ensemble local search can be described as follows:

1. Set $l=1$.

2. Sort all machines non-increasing based on the number of critical operations.

3. Set $m=1$.

4. Ensemble local search

4.1 Select a local search operator from the operator pool.

4.2 Generate neighbor solution.

4.3 If the neighbor solution is better than the current one, remain the operator; otherwise, delete the operator from operator pool.

5. Set $l=l+1$, if $l \leq L$ ( $L$ is the maximum iteration), repeat (4) until operator pool is empty; otherwise, stop and return solution.

6. If the solution is improved, update the solution and go to (2).

7. Set $m=m+1$, if $m \leq M$ ( $M$ is machine number), go to (4); otherwise, go to (3).

The computational complexity to get a non-increasing machine sequence is $O\left(M \log _{2} M\right)$ and the complexity to search local space is $O(M * L)$. Hence, the computational complexity of the ensemble local search is $O\left(M\left(\log _{2} M+L\right)\right)$.

\subsection{Two-stage $\mathrm{ABC}$ algorithm framework}

The TABC algorithm employs multiple initialization strategies, novel machine assignment and operation sequencing operator for generating new solutions, ensemble local search method to improve algorithm performance. The exploitation and exploration are balanced and stressed in this algorithm. 
The proposed TABC algorithm includes two stages: scheduling stage and re-scheduling stage. At scheduling stage, the start time of all jobs and machines is at time 0. After scheduling stage, the solution with the best objective value will be output, and all operations will be processed on the corresponding machines based on the best solution. On new job(s) coming and inserting into the shop floor, the re-scheduling stage will be activated and reschedule the new job(s) and the operations that are not yet started. All not yet started operations and new job(s) will proceed based on the rescheduling best solution. The computational procedure of TABC algorithm for scheduling and rescheduling can be described as follows:

Scheduling stage:

1. Set parameters, including the number of employed bees, the number of onlooker bees and the number of scout bees. In TABC algorithm, the number of employed bees equal to the number of food sources.

2. Initialize population with multiple strategies shown in Section 4.2, evaluate each solution and determine the best food source.

3. Employed bee phase. For all populations, repeat the following sub-steps:

3.1 For each pair of current solutions, generate two new solutions by using the strategy presented in Section 4.3.

3.2 Improve the two new solutions based on the ensemble local search method in Section 4.4.

3.3) If the new solutions are better than or equal to the current solutions, update the population.

4. Onlooker bee phase. For all populations, repeat the following sub-steps:

4.1 Select two food sources for two onlooker bees by using tournament selection.

4.2 Generate two new solutions for the two onlookers by using the strategy in Section 4.3.

4.3 Improve the two new solutions based on the ensemble local search method in Section 4.4.

4.4 If the new solutions are better than or equal to the current solutions, update the population.

5. Scout bee phase. If a solution has not been improved during the last limit number of trials, abandon it, generate a new solution randomly and execute the ensemble local search on it.

6. Update the best solution. If the termination criterion is reached, return the best solution; otherwise, go to step (3).

Re-scheduling stage:

7. Insert the new job(s) in the scheduled and executing sequence.

8. Calculate and record the start time of each job and each machine based on the inserting time.

9. For existing jobs, Re-calculate the number of operations that are not yet started. Add the new job(s) to the re-scheduling job set.

10. Execute re-scheduling. 
11. Link up the re-scheduling results to executing scheduling results based on the inserting time.

\subsection{Experiment setup}

To test the performance of the proposed TABC algorithm, extensive experimental evaluation and comparisons with existing algorithms are provided using well-known FJSSP benchmark sets. Two sets of instances are considered in this Chapter: (1) the first data set are five Kacem instances [140], (2) the second data set, called BRdata, is a set of 10 problems by Brandimare[141]. The Kacem benchmark set is composed of five instances with the size ranging from 4 jobs, 5 machines and 11 operations to 15 jobs, 10 machines and 60 operations. The Brandimare benchmark set includes 10 problems with the size ranging from 10 jobs, 6 machines and 55 operations to 20 jobs, 16 machines and 240 operations.

Table 5 - 1 The data of eight remanufacturing instances

\begin{tabular}{|c|c|c|c|c|c|}
\hline Instance & Inserting Order & Inserting time & Job Number & Machine Number & Operation number \\
\hline \multirow{3}{*}{1} & $\overline{0}$ & 0 & 5 & \multirow{3}{*}{4} & 23 \\
\hline & 1 & 10 & 1 & & 4 \\
\hline & 2 & 15 & 1 & & 5 \\
\hline \multirow{4}{*}{2} & 0 & 0 & 8 & \multirow{3}{*}{8} & 64 \\
\hline & 1 & 12 & 1 & & 8 \\
\hline & 2 & 24 & 1 & & 8 \\
\hline & 0 & 0 & 10 & \multirow{4}{*}{6} & 81 \\
\hline \multirow{3}{*}{3} & 1 & 15 & 1 & & 8 \\
\hline & 2 & 25 & 1 & & 7 \\
\hline & 3 & 35 & 1 & & 6 \\
\hline \multirow{3}{*}{4} & 0 & 0 & 10 & \multirow{3}{*}{10} & 100 \\
\hline & 1 & 15 & 2 & & 20 \\
\hline & 2 & 30 & 2 & & 20 \\
\hline \multirow{3}{*}{5} & 0 & 0 & 15 & \multirow{3}{*}{8} & 171 \\
\hline & 1 & 21 & 2 & & 18 \\
\hline & 2 & 47 & 2 & & 17 \\
\hline \multirow{4}{*}{6} & 0 & 0 & 15 & \multirow{4}{*}{10} & 185 \\
\hline & 1 & 14 & 2 & & 20 \\
\hline & 2 & 31 & 2 & & 19 \\
\hline & 3 & 44 & 2 & & 19 \\
\hline \multirow{3}{*}{7} & 0 & 0 & 20 & \multirow{3}{*}{10} & 308 \\
\hline & 1 & 21 & 3 & & 38 \\
\hline & 2 & 52 & 3 & & 32 \\
\hline \multirow{4}{*}{8} & 0 & 0 & 20 & \multirow{4}{*}{15} & 355 \\
\hline & 1 & 18 & 3 & & 42 \\
\hline & 2 & 34 & 3 & & 42 \\
\hline & 3 & 49 & 2 & & 25 \\
\hline
\end{tabular}

To test the performance of TABC for FJSSP with new job inserting and the three re-scheduling strategies provided in Section 5. 2, an instance set from remanufacturing enterprise is considered. This instance set includes eight instances with the size ranging from 5 jobs, 4 machines and 23 operations to 20 jobs, 15 machines and 355 operations. There are 35 new jobs inserted to existing scheduling 
sequence of eight instances. Each inserting has different inserting time, job number and operation number. The detail information is shown in Table $5-1$. The first two columns are instance number and new job insertion times. The third column is the inserting time of corresponding inserting order. The last three columns are the corresponding job number, machine number and operation number. The first row of each instance shows the job number, machine number and operation number at initial scheduling.

The TABC algorithm is coded in C++ and implemented on an Intel ${ }^{\circledR}$ Core TM2 Duo CPU P8600 @ 2.40GHZ PC with 4 GB RAM. The population size is fixed at 50. The maximum generation is 3000 . The probability of crossover operator is 0.4 . The maximum iteration of ensemble local search is set to 5 times machine number. Each instance is carried out 30 replications.

\subsection{Discussion and comparison of three re-scheduling strategies}

For the eight instances from remanufacturing enterprise, the re-scheduling is based on the initiating scheduling or last time re-scheduling for new job(s) inserting. In this section, we just focus on the minimal makespan over 30 runs. To compare the three re-scheduling strategies, we also calculated the relative percentage increase (RPI) as follows:

$$
\operatorname{RPI}\left(C_{M}^{i}\right)=\frac{C_{M}^{i}-C_{M}^{*}}{C_{M}^{*}} \times 100
$$

Where $C_{M}^{i}$ is the makespan value obtained in the ith replication, $C_{M}^{*}$ is the best maksespan value found by three re-scheduling strategies. Obviously, the smaller the RPI value, the better result the rescheduling strategy produces. To show the algorithm performance, we also calculated the average relative percentage increase (ARPI) of each re-scheduling strategy. For each instance, the average run time over 30 replications also recorded for the performance of TABC algorithm and three rescheduling strategies.

To compare three re-scheduling strategies detailed, the eight remanufacturing instances are divided into small-scale set and large-scale set. The small-scale set include instance 1 to instance 4 , and the larger set include instance 5 to instance 8. For each instance, the minimal makespan value (CM), the average run time over 30 times and the average relative percentage increase (ARPI) are shown in Table $5-2$ and Table $5-3$. The first row of each instance is the corresponding results of initiating scheduling, and the result values of three re-scheduling strategies are the same. The insert order of initiating scheduling is set as " 0 " while the each new job(s) inserting order is increased.

It can be easily seen from Table 5 - 2 and Table 5 - 3 that the re-scheduling strategy II was found to give the best makespan and ARPI values and for each new job(s) inserting. The re-scheduling strategy 
II also obtained average APRI for small-scale, 0.45, and large-scale, 0.75, instance sets. The rescheduling strategy I got about the same average APRI for small-scale, 31.39, and large-scale, 30.14, instance sets. The re-scheduling III obtained different average APRI for small-scale, 21.62 and largescale, 18.85, instance sets. The re-scheduling strategy III has smaller average APRI value for largescale instance set. Compared to re-scheduling I and III, the re-scheduling strategy II costs maximum summation-running time for all new job(s) inserting re-scheduling. The summation re-scheduling time for small-scale is 21.483 seconds, large-scale 279.157. The summation-running time of re-scheduling strategy I and III are no significant difference. Hence, the re-scheduling II found the best solutions with the maximum running time in three re-scheduling strategies. The re-scheduling strategy III obtained better solutions than re-scheduling strategy I with about the same time.

Table 5 - 2 Scheduling and re-scheduling results for small-scale instances

\begin{tabular}{|c|c|c|c|c|c|c|c|c|c|c|}
\hline \multirow{2}{*}{ Instance } & \multirow{2}{*}{$\begin{array}{r}\text { Inserting } \\
\text { Order }\end{array}$} & \multicolumn{3}{|c|}{ Re-sche I } & \multicolumn{3}{|c|}{ Re-sche II } & \multicolumn{3}{|c|}{ Re-sche III } \\
\hline & & $\mathrm{C}_{\mathrm{M}}$ & Time(s) & ARPI $(\%)$ & $\mathrm{C}_{\mathrm{M}}$ & Time(s) & $\operatorname{ARPI}(\%)$ & $\mathrm{C}_{\mathrm{M}}$ & Time(s) & ARPI $(\%)$ \\
\hline \multirow[t]{3}{*}{1} & 0 & 25 & 1.032 & 0.00 & 25 & 1.032 & 0.00 & 25 & 1.032 & 0.00 \\
\hline & 1 & 36 & 0.156 & 33.33 & 27 & 0.469 & 0.37 & 36 & 0.312 & 33.33 \\
\hline & 2 & 36 & 0.219 & 12.50 & 32 & 0.531 & 0.00 & 36 & 0.341 & 12.50 \\
\hline \multirow[t]{3}{*}{2} & 0 & 36 & 4.203 & 0.00 & 36 & 4.203 & 0.00 & 36 & 4.203 & 0.00 \\
\hline & 1 & 63 & 0.235 & 38.64 & 44 & 2.343 & 0.00 & 53 & 0.419 & 20.45 \\
\hline & 2 & 77 & 0.312 & 57.14 & 49 & 1.531 & 1.84 & 59 & 0.487 & 20.41 \\
\hline \multirow[t]{4}{*}{3} & 0 & 55 & 5.641 & 0.00 & 55 & 5.641 & 0.00 & 55 & 5.641 & 0.00 \\
\hline & 1 & 77 & 0.281 & 32.76 & 58 & 3.640 & 0.17 & 77 & 0.646 & 32.76 \\
\hline & 2 & 111 & 0.261 & 68.18 & 66 & 2.687 & 1.97 & 111 & 0.583 & 68.18 \\
\hline & 3 & 111 & 0.247 & 58.57 & 70 & 2.172 & 1.14 & 111 & 0.602 & 58.57 \\
\hline \multirow[t]{3}{*}{4} & 0 & 44 & 7.938 & 0.00 & 44 & 7.938 & 0.00 & 44 & 7.938 & 0.00 \\
\hline & 1 & 69 & 1.020 & 35.29 & 51 & 5.344 & 0.00 & 56 & 2.754 & 9.80 \\
\hline & 2 & 103 & 1.111 & 71.67 & 60 & 2.766 & 0.33 & 75 & 1.673 & 25.00 \\
\hline Avg & & & & 31.39 & & & 0.45 & & & 21.62 \\
\hline Sum & & & 3.842 & & & 21.483 & & & 7.817 & \\
\hline
\end{tabular}

To illustrate the three re-scheduling strategies more clearly, the first time new job inserting rescheduling of instance 2 is used as an example. Fig. 5 - 1 shows the initiating scheduling Gantt chart. The makespan value is 36. It means that all existing jobs will be completed and all machines are available at time " 36 " if there is no new job(s) inserting. The new job, Job 9, inserts at time "12". At this time, the machine release time, job release time and corresponding operation number are shown in Table 5 - 4. For example, the fourth operation of job 1, O1.4, is processing on machine M3 on time "12". This operation will be completed on time "13" on machine M3. Hence, the release time of job 1 is on time " 13 " and the start operation is O1.5. At the same time, the release time of machine M3 is 
also on time "13". In the same way, all job release time and machine can be obtained. For the new job $\mathrm{J} 9$, the release time is on time " 12 " and the first start operation is operation O9.1.

The three re-scheduling strategies' Gantt charts are shown in Fig. 5 - 2 to Fig. 5 - 4. Fig. 5 - 2 is the Gantt chart of re-scheduling strategy I. The new inserting job, Job 9 is re-scheduling from time " 36 " and the finial complete time is 63. Fig. 5 - 3 shows the Gantt chart of re-scheduling strategy II. The re-scheduled operations include Job9's operations and the existing jobs' operations that have not started at the inserting time "12". Compared to Fig. 5 - 1, the existing jobs' operations have different and new operation sequence. The Job9's operations are also included in the new operation sequences. The final complete time is 44 by re-scheduling strategy II. The Gantt chart by re-scheduling strategy III is shown in Fig. 5 - 4. It can be seen that the existing jobs' operations are the same with initial scheduling, shown in Fig. 5 - 1. The first three operations of Job 9 are inserted into the free-time-gap of existing operation sequence. The fourth operation's start time is time " 35 " which is also earlier than the makespan value, unit time " 36 ", in initial scheduling. The final complete time in Fig. 5 - 4 is 53. Table 5 - 3 Scheduling and re-scheduling results for large-scale instances

\begin{tabular}{|c|c|c|c|c|c|c|c|c|c|c|}
\hline \multirow{2}{*}{ Instance } & \multirow{2}{*}{$\begin{array}{r}\text { Inserting } \\
\text { Order }\end{array}$} & \multicolumn{3}{|c|}{ Re-sche I } & \multicolumn{3}{|c|}{ Re-sche II } & \multicolumn{3}{|c|}{ Re-sche III } \\
\hline & & $\mathrm{C}_{\mathrm{M}}$ & Time(s) & ARPI $(\%)$ & $\mathrm{C}_{\mathrm{M}}$ & Time(s) & ARPI (\%) & $\mathrm{C}_{\mathrm{M}}$ & Time(s) & ARPI (\%) \\
\hline \multirow[t]{3}{*}{5} & 0 & 79 & 19.813 & 0.38 & 79 & 19.813 & 0.38 & 79 & 19.813 & 0.38 \\
\hline & 1 & 106 & 0.904 & 29.27 & 82 & 10.891 & 0.98 & 101 & 1.657 & 23.17 \\
\hline & 2 & 132 & 0.817 & 48.31 & 89 & 5.391 & 1.12 & 114 & 1.490 & 28.09 \\
\hline \multirow[t]{4}{*}{6} & 0 & 73 & 25.157 & 0.00 & 73 & 25.157 & 0.00 & 73 & 25.157 & 0.00 \\
\hline & 1 & 97 & 1.008 & 32.88 & 73 & 18.875 & 1.37 & 97 & 1.369 & 32.88 \\
\hline & 2 & 115 & 0.939 & 49.35 & 77 & 11.250 & 1.04 & 110 & 1.425 & 42.86 \\
\hline & 3 & 118 & 0.931 & 51.28 & 78 & 8.594 & 1.28 & 110 & 2.247 & 41.03 \\
\hline \multirow[t]{3}{*}{7} & 0 & 96 & 53.594 & 0.31 & 96 & 53.594 & 0.31 & 96 & 53.594 & 0.31 \\
\hline & 1 & 140 & 2.423 & 26.13 & 111 & 41.391 & 0.45 & 140 & 3.163 & 26.13 \\
\hline & 2 & 168 & 1.807 & 37.70 & 122 & 25.391 & 0.82 & 140 & 6.592 & 14.75 \\
\hline \multirow[t]{4}{*}{8} & 0 & 91 & 112.078 & 0.66 & 91 & 112.078 & 0.66 & 91 & 112.078 & 0.66 \\
\hline & 1 & 116 & 2.865 & 24.73 & 93 & 71.578 & 0.00 & 104 & 4.036 & 11.83 \\
\hline & 2 & 147 & 2.800 & 51.55 & 97 & 53.156 & 1.03 & 109 & 4.317 & 12.37 \\
\hline & 3 & 161 & 2.237 & 69.47 & 95 & 32.640 & 1.05 & 123 & 3.941 & 29.47 \\
\hline Sum $^{\text {Avg }}$ & & & 16.731 & 30.14 & & 279.157 & 0.75 & & 30.237 & 18.85 \\
\hline
\end{tabular}

Table 5 - 4 Machine release time, job release time and corresponding operations

\begin{tabular}{lrrrrrrrrr}
\hline Order & 1 & 2 & 3 & 4 & 5 & 6 & 7 & 8 & 9 \\
\hline Job release time & 13 & 12 & 17 & 13 & 19 & 18 & 13 & 16 & 12 \\
Job start operation & 1.5 & 2.4 & 3.3 & 4.4 & 5.5 & 6.6 & 7.6 & 8.4 & 9.1 \\
Machine release time & 16 & 17 & 13 & 18 & 19 & 12 & 13 & 13 & \\
\hline
\end{tabular}


It is clear that the re-scheduling strategy II is more appropriate than re-scheduling strategy III if the new Job 9 has the same priority with the existing jobs. The re-scheduling strategy III should be selected if the existing jobs are priority completed. In practical remanufacturing environment, re-scheduling strategy III may also be selected if the operations of existing jobs cannot be moved from assigned machine. Although three re-scheduling obtain different performance, each of them may be selected in practical shop floor.

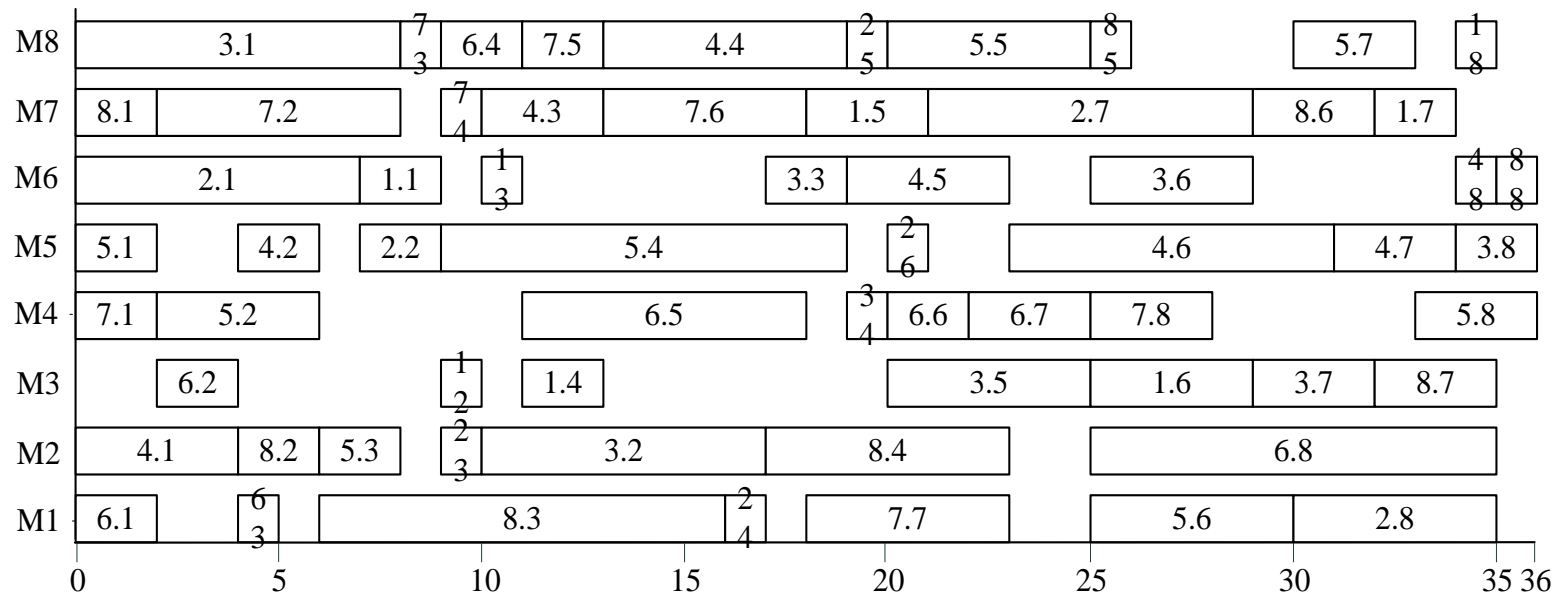

Fig. 5 - 1 The Gantt chart of initial scheduling

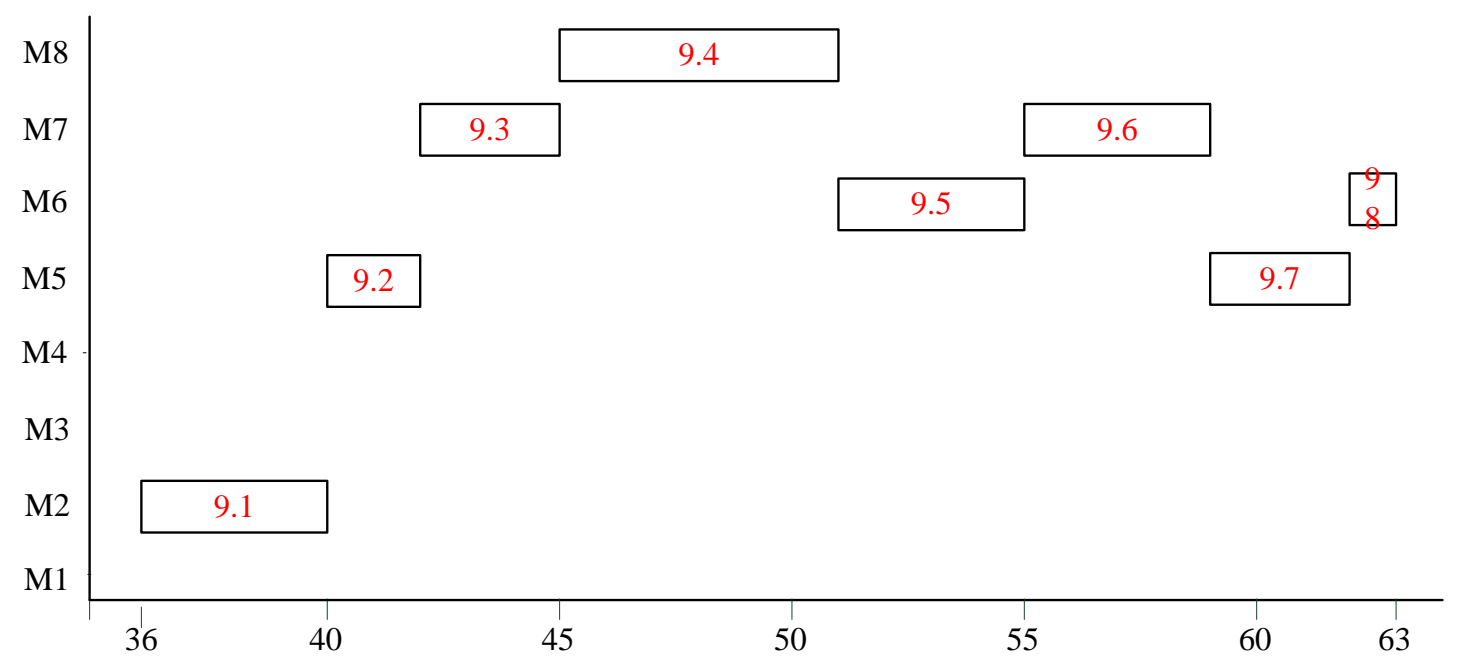

Fig. 5 - 2 The Gantt chart by re-scheduling strategy I 


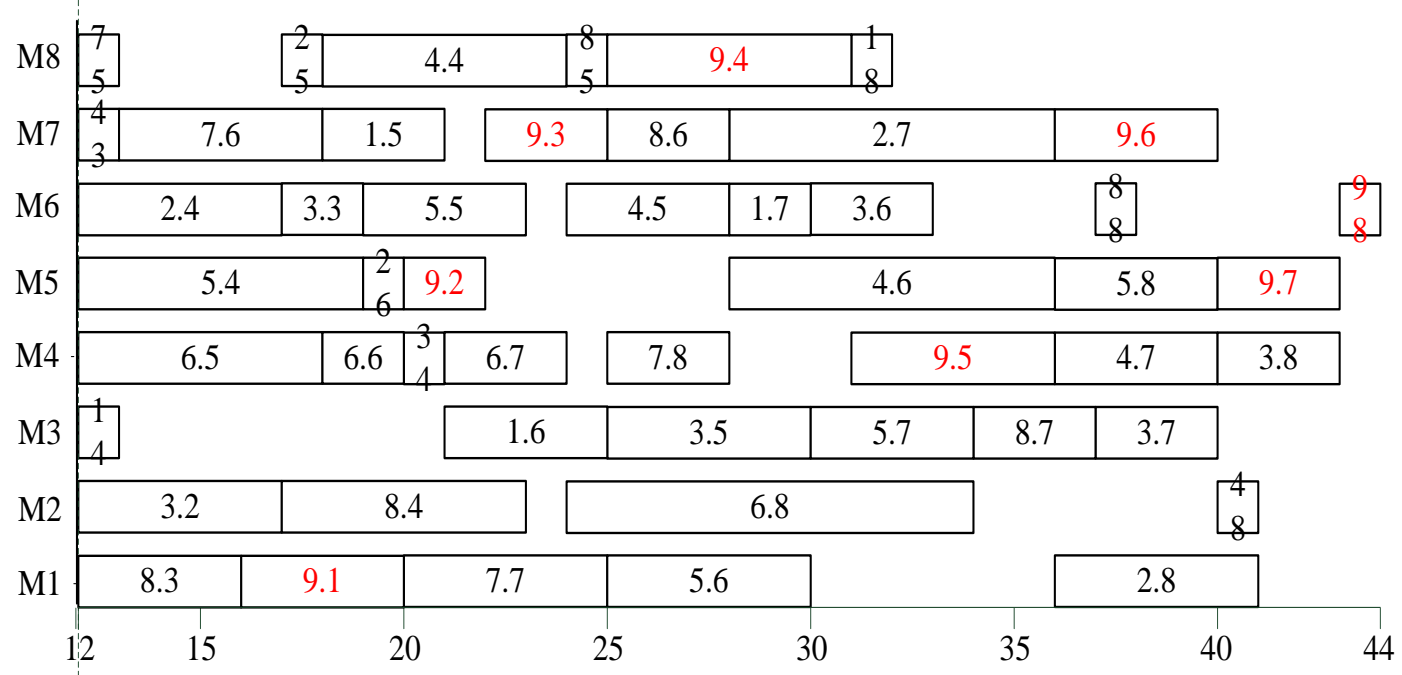

Fig. 5 - 3 The Gantt chart by re-scheduling strategy II

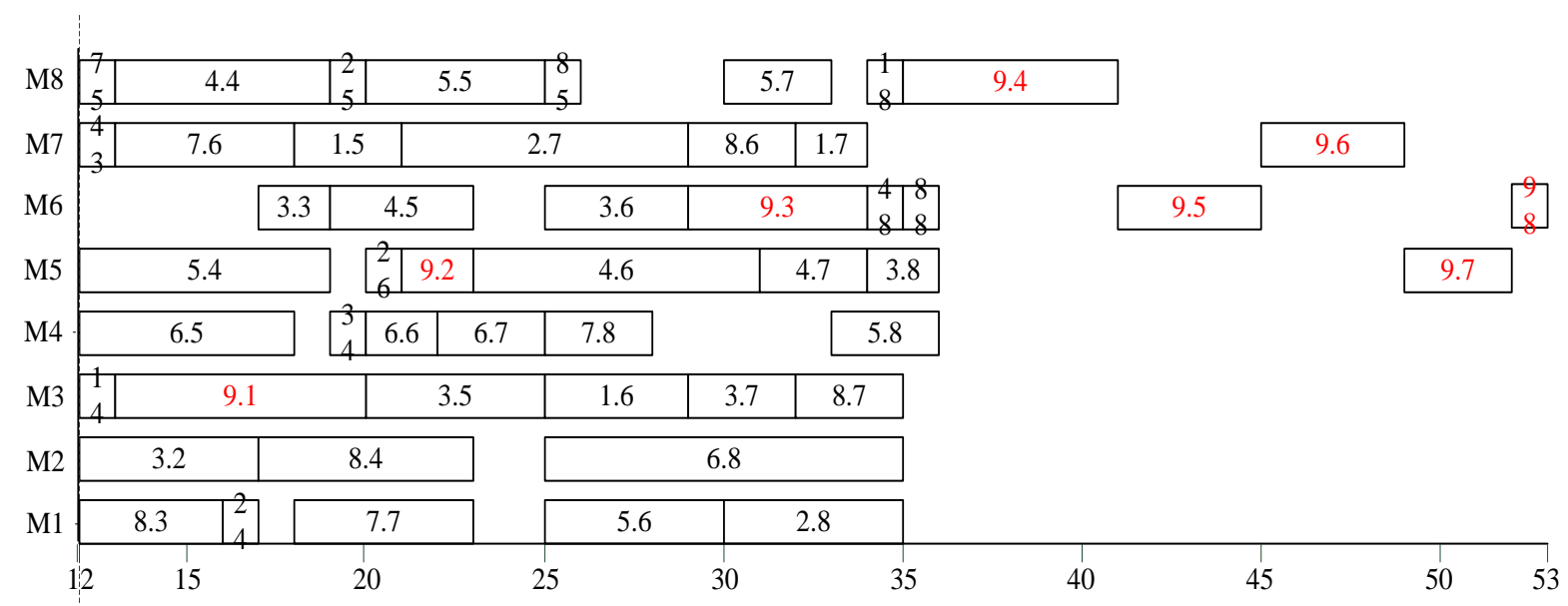

Fig. 5 - 4 The Gantt chart by re-scheduling strategy III

\subsection{Comparison with existing algorithms}

In scheduling phase, the job start time and machine release time are the same, zero. Hence, the problem is a general FJSSP. In re-scheduling phase, the job start time may be different and the machine release time may be different. The problem becomes FJSSP with different job start time and different machine release time. In addition, the job number is also different in different re-scheduling stages. In this section, we compare the TABC algorithm to several existing algorithms for FJSSP in scheduling phase. For the re-scheduling phase, we compare $\mathrm{TABC}$ algorithm with several simple and combination heuristics. The results and discussions are shown in sections 5.7.1 and 5.7.2.

\subsubsection{Scheduling stage}


There are many existing meta-heuristics for solving flexible job shop scheduling problem with makespan criterion. To test the performance of proposed algorithm, we compare TABC algorithm to five competitive meta-heuristics, parallel variable neighborhood search (PVNS), knowledge-based ant colony optimization (KBACO) [22], tabu search algorithm with efficient neighborhood structure (TSPCB) [71], effective artificial bee colony algorithm (EABC) [136] and a simple and effective evolutionary algorithm (SEA) [51]. TABC algorithm is also compared to the DHS algorithm proposed in Chapter 4. The makespan values for all the algorithms are shown in Table 5 - 5. For five Kacem instances, all compared algorithms can obtain the best known makespan as long as the instances were solved. The only exception is that PVNS did not reach the best-known makespan for Kacme 5 with 15 jobs, 10 machines and 60 operations. For 10 BRdata instance, TABC and EABC are the two best algorithms. TABC obtained minimal makespan values for eight instances except MK01 and MK05 while EABC also reached eight minimal makespan values except instance MK01 and MK10. PVNS found minimal makspan for six instances, and TSPCB did it for four instances. Both KBACO and SEA found 3 minimal makespn values in 10 instances. Compare to DHS algorithm, TABC algorithm improves the results of instances 6, 7, 9 and 10. The result of instance 5 by TABC is larger than that by DHS algorithm. TABC algorithm is more competitive for large-scale instances. For each instance, the standard deviation of makespan value over 30 runs is shown in the second last column of Table 5 - 5. All the compared algorithms are run on different PC with different parameters and running time. In this Chapter, we just provide the average running time over 30 times of the proposed TABC algorithm to find the minimal makespan for corresponding instances. The detail information of running time for all benchmark instances is also shown in the last column of Table 5 - 5. Based on the makespan results of TABC and comparison to existing algorithms, the TABC algorithm is effective and efficiency for solving the flexible job shop scheduling problem. Hence, the TABC algorithm is also employed for re-scheduling when new job(s) come(s) and insert(s) into scheduled operation sequence.

Table 5 - 5 Results of two benchmark sets by all compared algorithms

\begin{tabular}{|c|c|c|c|c|c|c|c|c|c|}
\hline \multirow{2}{*}{ Instance } & \multirow{2}{*}{ PVNS } & \multirow{2}{*}{ KBACO } & \multirow{2}{*}{ TSPCB } & \multirow{2}{*}{ EABC } & \multirow{2}{*}{ SEA } & \multirow{2}{*}{ DHS } & \multicolumn{3}{|c|}{ TABC } \\
\hline & & & & & & & $\mathrm{C}_{\mathrm{M}}$ & SD & Avg_t (s) \\
\hline Kacme1 & N/A & 11 & 11 & 11 & 11 & 11 & 11 & 0.00 & 0.47 \\
\hline Kacme 2 & 14 & 14 & 14 & 14 & 14 & 14 & 14 & 0.00 & 1.19 \\
\hline Kacme 3 & N/A & 11 & 11 & 11 & 11 & 11 & 11 & 0.00 & 1.2 \\
\hline Kacme 4 & 7 & 7 & 7 & 7 & 7 & 7 & 7 & 0.00 & 1.4 \\
\hline Kacme 5 & 12 & 11 & 11 & 11 & 11 & 11 & 11 & 0.00 & 2.97 \\
\hline MK01 & 40 & 39 & 40 & 40 & 40 & 40 & 40 & 0.53 & 3.36 \\
\hline MK02 & 26 & 29 & 26 & 26 & 26 & 28 & 26 & 0.81 & 3.72 \\
\hline
\end{tabular}




\begin{tabular}{lrrrrrrrrr}
\hline MK03 & 204 & 204 & 204 & 204 & 204 & 204 & 204 & 0.00 & 1.56 \\
MK04 & 60 & 65 & 62 & 60 & 61 & 60 & 60 & 0.83 & 66.58 \\
MK05 & 173 & 173 & 172 & 172 & 173 & 172 & 173 & 0.00 & 78.45 \\
MK06 & 60 & 67 & 65 & 60 & 65 & 67 & 60 & 2.02 & 173.98 \\
MK07 & 141 & 144 & 140 & 139 & 140 & 143 & 139 & 0.91 & 66.19 \\
MK08 & 523 & 523 & 523 & 523 & 523 & 523 & 523 & 0.00 & 2.15 \\
MK09 & 307 & 311 & 310 & 307 & 311 & 309 & 307 & 1.81 & 304.43 \\
MK10 & 208 & 229 & 214 & 208 & 225 & 212 & 202 & 4.98 & 418.19 \\
No. C & $2+6$ & $5+3$ & $5+4$ & $5+8$ & $5+3$ & $5+4$ & $5+8$ & & \\
\hline
\end{tabular}

\subsubsection{Re-scheduling for new job inserting}

Several simple heuristics are proposed for machine assignment and operation sequencing in FJSSP. For example, machine with minimum workload heuristic (MSC) is for machine assignment while job with maximum remaining work (MSB) and job with maximum remain operations (MSC1) are two heuristics for operation sequencing. In this section, we improve some simple heuristics and develop combined heuristics based on simple heuristics. For example, random job order in machine with minimum workload heuristic (RMSC), MSC+MSB heuristic, RMSC+MSB heuristic, MSC+MSC1 heuristic, RMSC+MSC1 heuristic etc al. we also combine MSC1 heuristic with RH heuristic proposed in Section 4.2. MSC1 heuristic is employed to obtain the operation sequence in RH heuristic. Based on the initial scheduling results of simple heuristics and the combination heuristics, we compare TABC algorithm to MSC, RMSC, RMSC+MSB, RMSC+MSC1, RH and MSC1+RH heuristics in rescheduling phase. The initializing scheduling and all re-scheduling results are shown in Table 5 - 6 . MSC and MSC1 are simple heuristics and the results are the fixed. MSC1+RH heuristic also have fixed results. RMSC, RMSC+MSB, RMSC+MSC1 and RH heuristics run 30 times. We count minimum values (min), average values (ave) and standard deviation results by these four heuristics. $\mathrm{TABC}$ algorithm is also run 30 times and the results are fixed for the same instance. For each instance, the best solution is selected for next rescheduling operator.

It can be seen from Table 5 - 6 that MSC has the worst average (Ave) result for eight instances. RMSC and RMSC+MSB obtain quite minimum (min) values, average (ave) values and standard deviation (SD) values for initializing scheduling and all re-scheduling operators. RMSC+MSC1, MSC1+RH and RH have similar average values of min, ave and SD, RMSC+MSC1 (85.8, 93.6, 4.5), MSC1+RH (84.3) and RH $(81.3,90.3,5.0)$. In these three heuristics, RH has the best Ave value of min (81.3) and the worst average value of SD (5.0). For all initializing scheduling and all re-scheduling operators, TABC obtain best results and best Ave value. Among all compared heuristics, only RH can find the best result of instance 1 at initializing phase. 
For all compared algorithms, there are several special results. For example, MSC1+RH heuristic obtains a smaller first re-scheduling minimum result (71) than the initial result (77) for the second instance. It means that the re-scheduling results have much improvement after inserting the first new job into the second instance. MSC1+RH show the same situation for the fourth instance and the eighth instance. In addition, this heuristic has the same initial scheduling and the first re-scheduling results (100) for the eighth instance. TABC algorithm has the same initial scheduling and the first rescheduling results (73) for the sixth instance. In the third re-scheduling operator for the eighth instance, TABC obtains a smaller result (95) than the second re-scheduling result (97). These situations mean that, after inserting new job, the re-scheduling operator may remain or even reduce makespan value in some special situation. To compare different heuristics and TABC algorithm clearly, Fig. 5 - 5 and Fig. 5 - 6 show the initial scheduling results and all re-scheduling results for the sixth instance and eighth instance. It is clear that TABC has better results than other heuristics in initial scheduling and re-scheduling operator phase.

In summary, TABC algorithm is effective and efficiency for solving FJSSP. For scheduling and rescheduling FJSSP with new job inserting, combination heuristics and RH has better performance than simple heuristics. TABC algorithm obtains better results than simple and combination heuristics in both initial scheduling and re-scheduling phase.

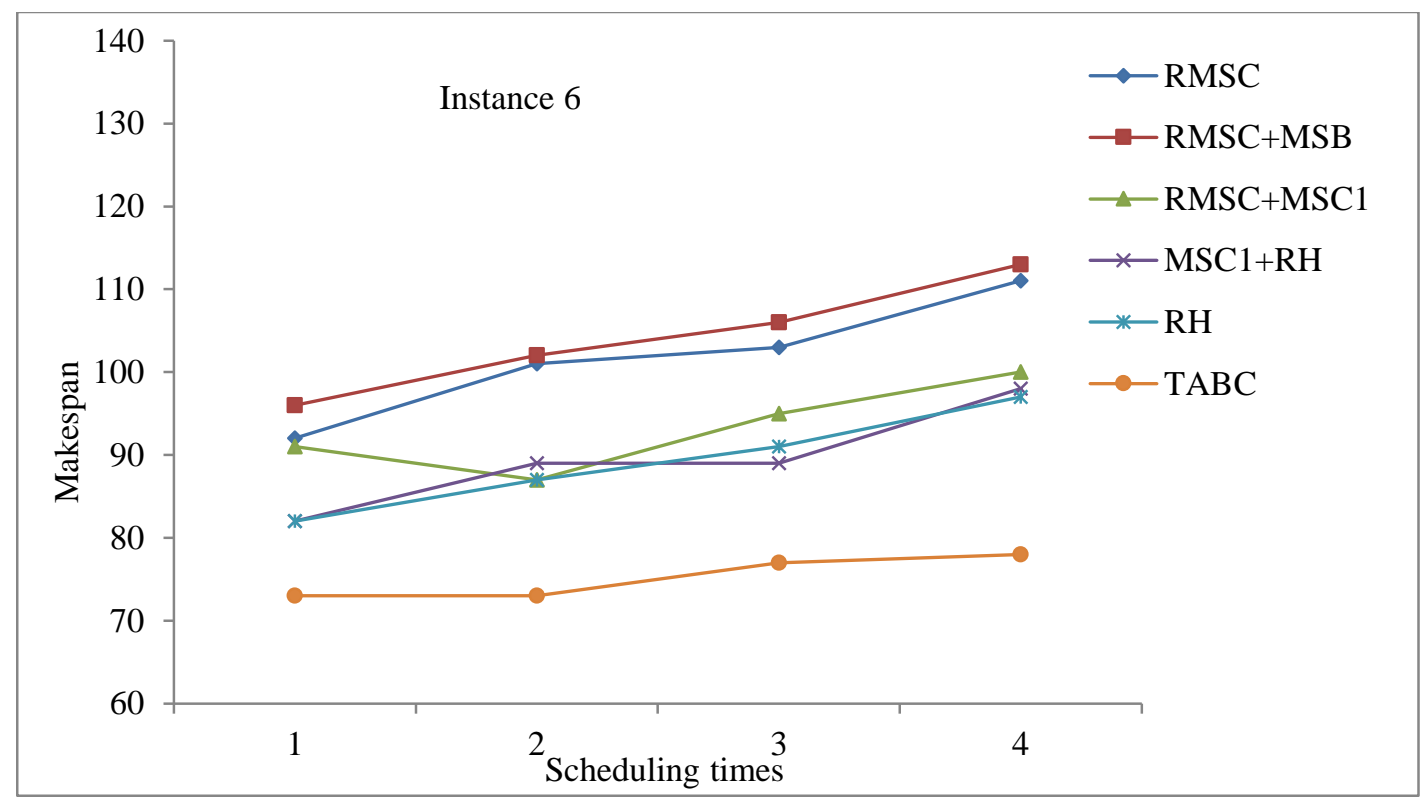

Fig. 5 - 5 Scheduling and re-scheduling results for instance 6 
Table 5 - 6 Scheduling and rescheduling results

\begin{tabular}{|c|c|c|c|c|c|c|c|c|c|c|c|c|c|c|c|c|}
\hline \multirow{2}{*}{ Instance } & \multirow{2}{*}{$\begin{array}{l}\text { Insertion } \\
\text { Order }\end{array}$} & \multirow{2}{*}{ MSC } & \multicolumn{3}{|c|}{ RMSC } & \multicolumn{3}{|c|}{ RMSC+MSB } & \multicolumn{3}{|c|}{ RMSC+MSC1 } & \multirow{2}{*}{$\begin{array}{l}\mathrm{MSC} 1 \\
+\mathrm{RH}\end{array}$} & \multicolumn{3}{|c|}{ RH } & \multirow{2}{*}{ TABC } \\
\hline & & & $\min$ & ave & SD & $\min$ & ave & SD & $\min$ & ave & SD & & $\min$ & ave & SD & \\
\hline \multirow{3}{*}{1} & 0 & 39 & 27 & 34.3 & 4.03 & 28 & 35.6 & 3.81 & 27 & 29.4 & 2.77 & 28 & 25 & 33 & 4.39 & 25 \\
\hline & 1 & 43 & 31 & 34.3 & 1.75 & 31 & 33.4 & 1.77 & 34 & 35.4 & 1.61 & 36 & 28 & 30.6 & 2.42 & 27 \\
\hline & 2 & 56 & 34 & 39.7 & 3.41 & 35 & 40.2 & 3.45 & 35 & 38.1 & 2.07 & 44 & 33 & 37.2 & 3.59 & 32 \\
\hline \multirow{3}{*}{2} & 0 & 90 & 48 & 57.3 & 5.66 & 50 & 58.9 & 6.04 & 49 & 58.7 & 5.06 & 51 & 44 & 51.6 & 3.12 & 36 \\
\hline & 1 & 107 & 55 & 64.1 & 6.32 & 56 & 64.7 & 3.91 & 57 & 64.2 & 3.63 & 55 & 52 & 58.4 & 3.82 & 44 \\
\hline & 2 & 102 & 58 & 63.2 & 2.62 & 60 & 67.7 & 4.79 & 63 & 68.4 & 4.15 & 62 & 56 & 63 & 5.37 & 49 \\
\hline \multirow{4}{*}{3} & 0 & 106 & 70 & 81.8 & 7.01 & 71 & 82.1 & 4.78 & 69 & 78.1 & 4.97 & 77 & 67 & 80.9 & 7.34 & 55 \\
\hline & 1 & 123 & 73 & 80.3 & 3.52 & 74 & 80.8 & 4.27 & 71 & 80.1 & 5.75 & 71 & 71 & 81.6 & 6.04 & 58 \\
\hline & 2 & 131 & 84 & 94.6 & 4.87 & 88 & 97.1 & 4.77 & 78 & 86.8 & 4.71 & 80 & 80 & 91.6 & 5.61 & 66 \\
\hline & 3 & 145 & 89 & 97.8 & 3.87 & 91 & 100.6 & 4.69 & 86 & 90.7 & 4.27 & 81 & 86 & 94.6 & 4.49 & 70 \\
\hline \multirow{3}{*}{4} & 0 & 111 & 61 & 70.1 & 5.63 & 63 & 69.8 & 4.2 & 58 & 69.7 & 5.92 & 67 & 56 & 67.4 & 5.94 & 44 \\
\hline & 1 & 116 & 69 & 75.8 & 5.04 & 70 & 79.7 & 4.77 & 67 & 76.7 & 5.57 & 64 & 61 & 71.7 & 4.59 & 51 \\
\hline & 2 & 140 & 84 & 94.4 & 7.73 & 83 & 93.8 & 4.61 & 81 & 88.4 & 4.91 & 84 & 74 & 84.7 & 5.97 & 60 \\
\hline \multirow{3}{*}{5} & 0 & 195 & 103 & 115.7 & 6.09 & 102 & 115.2 & 7.07 & 97 & 106 & 3.95 & 92 & 93 & 102.4 & 5.31 & 79 \\
\hline & 1 & 199 & 115 & 125.5 & 5.95 & 112 & 122.3 & 5.56 & 110 & 115.9 & 4.15 & 101 & 101 & 111.3 & 5.63 & 82 \\
\hline & 2 & 216 & 124 & 131.5 & 5.43 & 124 & 131.8 & 4.87 & 113 & 125 & 6.03 & 117 & 110 & 119.2 & 3.67 & 89 \\
\hline \multirow{4}{*}{6} & 0 & 174 & 92 & 107.1 & 7.17 & 96 & 110.5 & 5.96 & 91 & 96.3 & 4.55 & 82 & 82 & 91.3 & 5.98 & 73 \\
\hline & 1 & 184 & 101 & 111.5 & 7.18 & 102 & 110.9 & 5.85 & 87 & 99.1 & 5.78 & 89 & 87 & 95.6 & 5.13 & 73 \\
\hline & 2 & 192 & 103 & 112.8 & 5.43 & 106 & 116.3 & 4.29 & 95 & 101.6 & 4.28 & 89 & 91 & 102.9 & 5.04 & 77 \\
\hline & 3 & 220 & 111 & 117.2 & 4.29 & 113 & 123.4 & 6.64 & 100 & 106.9 & 4.48 & 98 & 97 & 105.1 & 6.53 & 78 \\
\hline \multirow{3}{*}{7} & 0 & 263 & 122 & 137.3 & 6.81 & 119 & 134.7 & 8.41 & 120 & 130.9 & 3.64 & 117 & 117 & 124.4 & 4.77 & 96 \\
\hline & 1 & 267 & 142 & 152.6 & 4.97 & 143 & 153.9 & 6.24 & 137 & 145.6 & 5.86 & 126 & 131 & 140.4 & 5.56 & 111 \\
\hline & 2 & 303 & 152 & 165.9 & 6.62 & 153 & 163.9 & 5.26 & 144 & 152.6 & 5.16 & 136 & 140 & 147.8 & 4.63 & 122 \\
\hline \multirow{4}{*}{8} & 0 & 223 & 110 & 121.2 & 5.16 & 111 & 121.1 & 7.12 & 99 & 110.7 & 5.29 & 100 & 94 & 106.2 & 7.24 & 91 \\
\hline & 1 & 258 & 118 & 126.5 & 4.7 & 119 & 126.8 & 5.69 & 108 & 117.1 & 4.86 & 100 & 101 & 106.7 & 3.83 & 93 \\
\hline & 2 & 275 & 134 & 141.1 & 4.05 & 130 & 139.4 & 5.45 & 118 & 126.6 & 4.47 & 116 & 108 & 117.5 & 4.93 & 97 \\
\hline & 3 & 268 & 134 & 140.9 & 5.04 & 137 & 143 & 4.88 & 123 & 130.1 & 4.05 & 114 & 110 & 121.3 & 4.97 & 95 \\
\hline Ave & & 168.3 & 90.5 & 99.8 & 5.2 & 91.3 & 100.6 & 5.1 & 85.8 & 93.6 & 4.5 & 84.3 & 81.3 & 90.3 & 5.0 & 69.3 \\
\hline
\end{tabular}




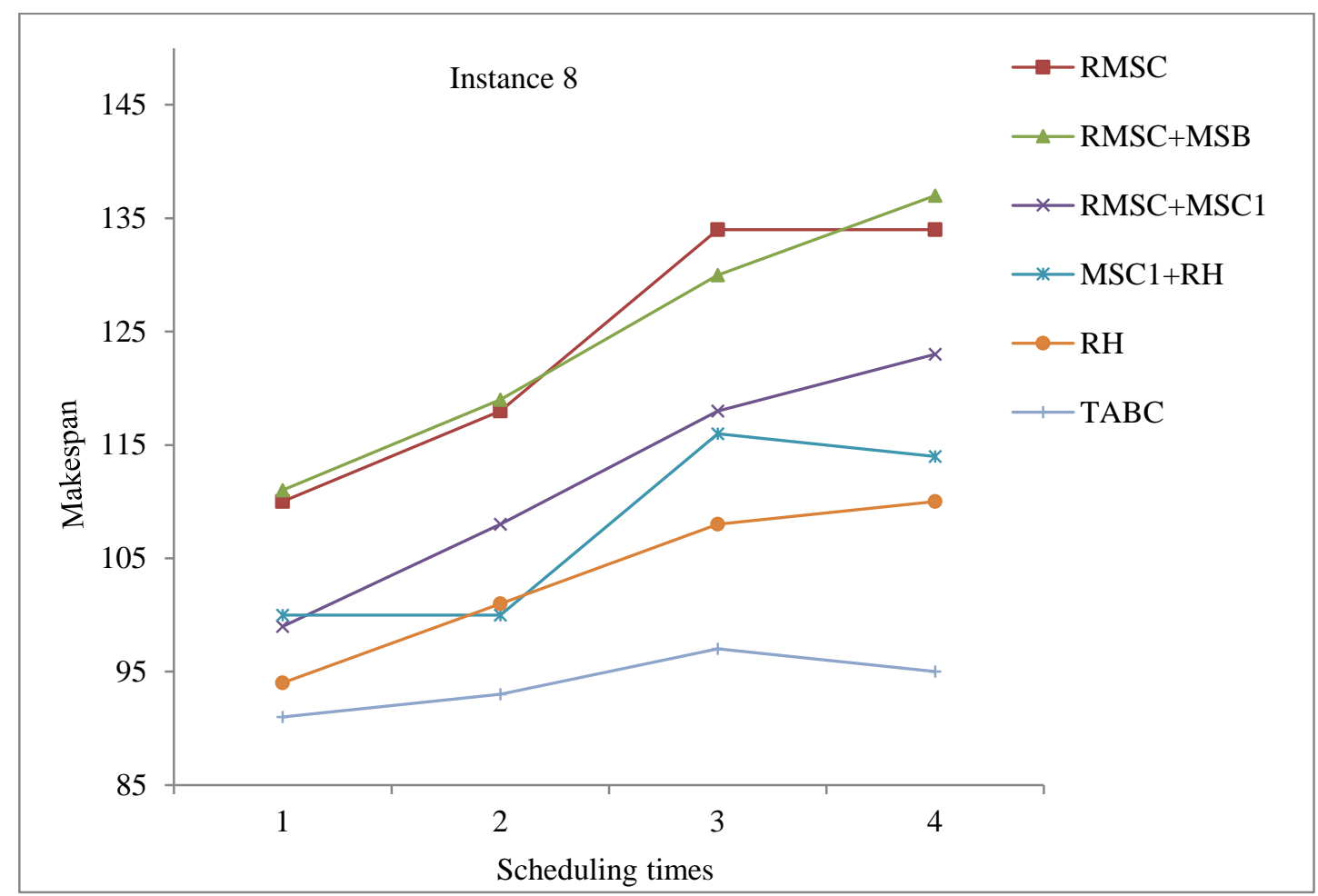

Fig. 5 - 6 Scheduling and re-scheduling results for instance 8

\subsection{Conclusions}

This Chapter proposed a two-stage hybrid artificial bee colony algorithm to solve flexible job shop rescheduling problem with the arrival of new jobs. The objective of this Chapter was to minimize makespan (maximum completion time). Three re-scheduling strategies were proposed for rescheduling new jobs. The three re-scheduling strategies were compared in terms of the re-scheduling performance. The proposed algorithm compared against several existing algorithms on scheduling performance. The experimental results showed the effectiveness and efficiency of the proposed algorithm for solving the flexible job shop scheduling problem. For the re-scheduling performance, TABC algorithm compared against several simple heuristics and the proposed combined heuristics. The results and comparisons demonstrated improved the performance of TABC algorithm. 


\section{Chapter 6}

\section{Two-stage DHS Algorithm for FJSSP with Multiple Constraints}

\subsection{Introduction}

Remanufacturing is a form of product recovery process and very important in waste management, material recovery and sustainable manufacturing. This Chapter researches a case study for the scheduling and rescheduling problem with processing time increasing and new job insertion constraints. The scheduling and rescheduling problems for re-processing are modeled as FJSSP with two constraints. This Chapter uses MAOS encoding and decoding method to represent a solution. An experience-based strategy is used to tackle the unpredictability of job processing time. Rescheduling strategy is considered for (1) processing time increasing and (2) new job insertion. A two-stage discrete harmony search (TDHS) algorithm based on problem characteristics is proposed for scheduling and rescheduling FJSSP. A local search method is employed for speeding up the convergence of TDHS. Two instances from a pump remanufacturing enterprise are solved. The objectives are to minimize the maximum completion time (makespan) and the mean of earliness and tardiness $(\mathrm{E} / \mathrm{T})$. These objectives are considered individually as well as together as a multi-objective 
problem. As a case study, we select two instances from remanufacturing engineering and describe the solving in detail. The specific information of the two instances are provided. Computational results show that the proposed TDHS algorithm can solve the real remanufacturing problems effectively. Scheduling and rescheduling results are satisfactory and can be used in practice.

The rest of this Chapter is organized as follows: Section 6.2 introduces the processing time increasing and new job insertion constraints in remanufacturing. The rescheduling strategy is proposed in Section 6.3. Section 6.4 gives the detailed computational procedure of the two-stage DHS algorithm for scheduling and rescheduling. Section 6.5 sets up experiments. Section 6.6 is devoted to computational results obtained from real data and comparisons to existing approaches. We conclude this Chapter in Section 6.7.

\subsection{Processing time increasing and new job insertion}

Fig. 6 - 1 shows one scheduling solution with processing time increasing. The dotted rectangle after operation V2.1 is the increasing processing time of job V2.1 in practice. Because this change of job V2.1 affects the makespan criterion in this instance, the operations after V2.1 should be rescheduled.

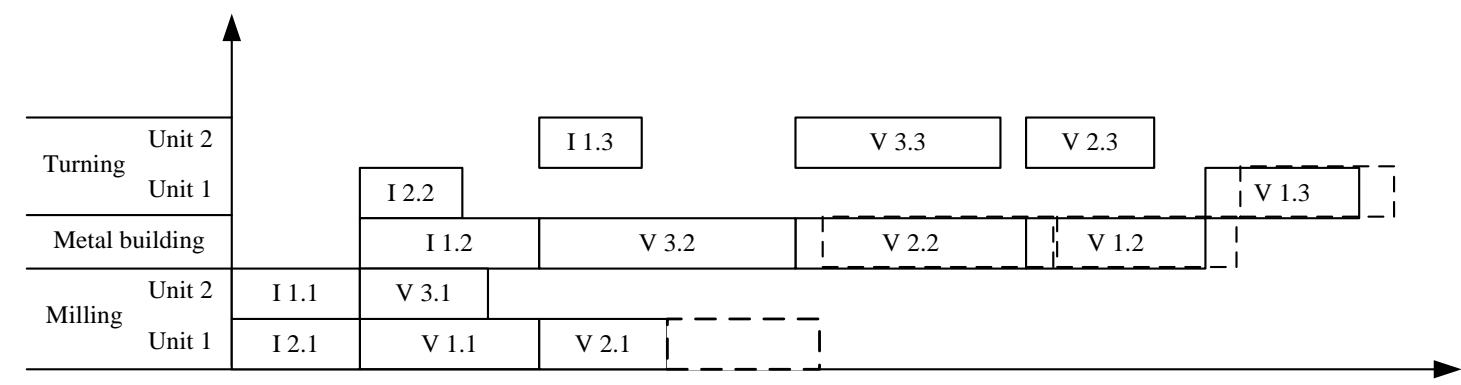

Fig. 6 - 1 A Gantt chart for increasing processing time

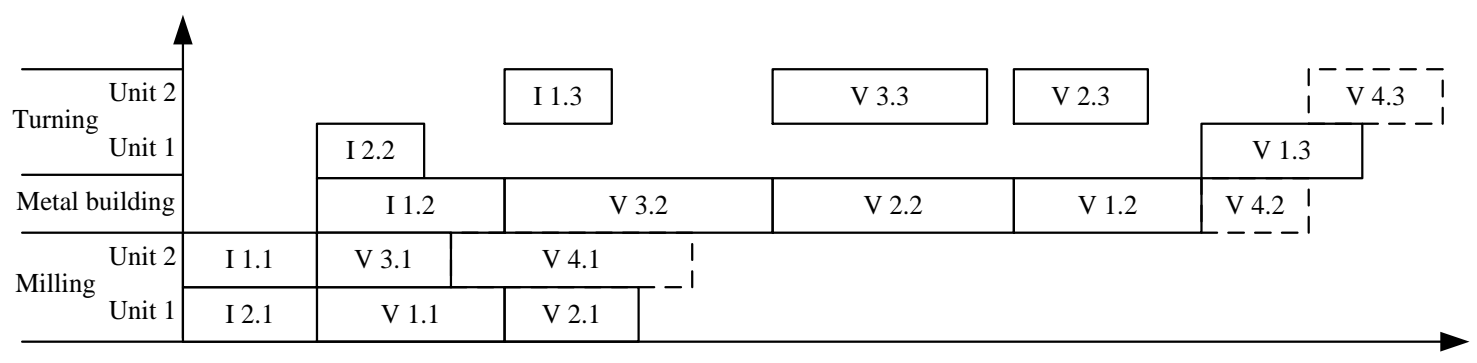

Fig. 6 - 2 A Gantt chart for new job insertion

The arrival of new products is also unpredictable in remanufacturing. When a new job inserts into the executing job sequence, the objective value and level satisfaction may also be affected. In this condition, we reschedule the operations that have not yet started at the insertion time. Fig. 6 - 2 shows 
an example of inserting a new incoming job into the schedule and executing sequence. The job Volute 4 is inserted into the scheduled sequence. Obviously, the insertion of Volute 4 affects the value of makespan. The operations that have not started at the insertion time should be rescheduled.

\subsection{Rescheduling strategy}

Rescheduling is for processing time increasing or new job insertion when the schedule is being executed. There are two strategies for these two stochastic unexpected situations: (1) The processing time increasing or new job insertion does not affect the value of objective nor satisfaction. There is no rescheduling for the executing sequence. (2) The processing time increasing or new job insertion affects the value of objective or satisfaction level. The remaining operations that have not yet started will be rescheduled for improving results. As shown in Fig. 6 - 3, the value of makespan becomes larger after inserting job Volute 4 into the scheduled sequence. To obtain a better value of makespan, we should reschedule the operations which have not yet started at insertion time. The corresponding operations are filled with lines. Rescheduling results have to link up to the executing scheduling results. Therefore, the available time of each machine and start time of each job in rescheduling must be recorded previously.

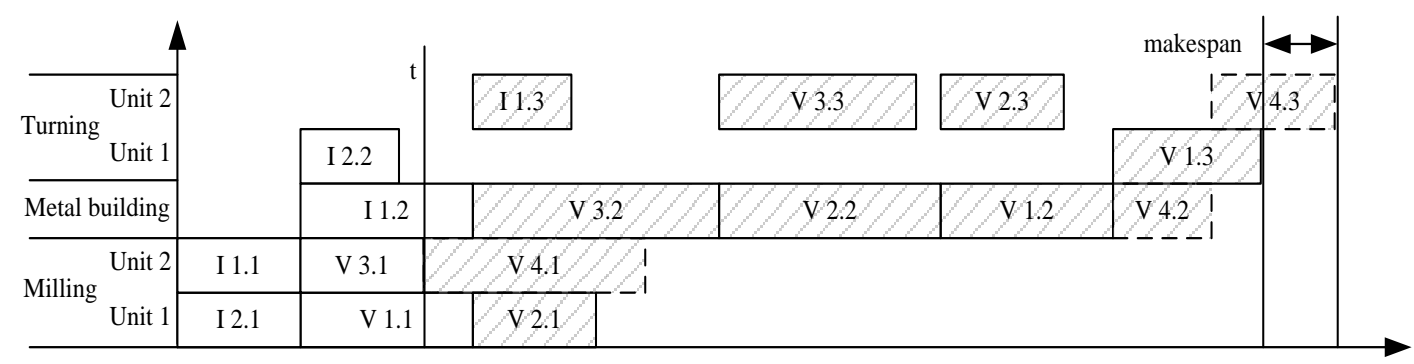

Fig. 6 - 3 Gantt chart for rescheduling

\subsection{Computational procedure}

The two-stage DHS algorithm employed dynamic grouping and local search operators in Chapter 4 to improve performance. The computational procedure of the two-stage DHS algorithm for scheduling and rescheduling can be described as follows:

Scheduling stage:

1) Initializing algorithm parameters and harmony memory.

2) Grouping harmony memory into many small Sub-HMs.

3) Sub-HM optimization

4) Improvising a new harmony. 
5) Local search for a new harmony.

6) Update Sub-HM.

7) Repeat step 4) to step 6) till all Sub-HMs are executed.

8) If the termination condition is not met, go to step 9); otherwise, go to step 11).

9) All Sub-HMs form HM and corresponding results are recorded.

10) Repeat step 2) to step 9).

11) Output the final scheduling results.

Rescheduling stage:

12) The processing time inceasing or new job insertion in the executing sequence.

13) If the objective values are affected, go to step 14); otherwise, continue processing in practice.

14) Reinitializing the avaliable time of all machines and recording the operations that have not yet started.

15) Rescheduling the operations which have not yet started at the insertion time.

16) Link up the rescheduling results to executing scheduling results.

For the two-stage DHS algorithm, the computational complexity of the first stage is the same to the complexity of DHS in Chapter 4. When the processing time increases or new job is inserted into the executing sequence, the first stage will be executed. Hence, the computational complexity of the twostage DHS algorithm is based on the number of times of processing time increasing or new job insertion.

\subsection{Experimental setup}

Table 6 - 1 and Table 6 - 2 show the jobs and the corresponding processing times in two Orders from a pump remanufacturing enterprise. In Table 6 - 1, there are two Volutes, two Impellers, one Shaft, two Shaft sleeves, three Case wear rings, and two Impeller keys. In Table 6 - 2, the jobs include two Volutes, three Impellers, two Shafts, three Shaft sleeves, four Case wear rings, three Impeller wear rings and three Impeller keys. Although the exact processing time of each job is unpredictable, experienced engineers can estimate it in advance. In Table $6-1$ and Table $6-2$, the processing times are the most probable processing time evaluated by engineers' experiences. In practice, if the processing time is larger than the most probable processing time, rescheduling will be executed.

In HS algorithm, the parameters HMS, HMCR and PAR affect the performance of HS algorithm. The HMS should be small. Omran [114] suggested that it was generally better to use a large value of HMCR (i.e., >=0.9). A large PAR value enhances the local exploitation ability of the algorithm while a small PAR value enlarges the search area and diversity of the HS algorithm. In this Chapter, the 
parameters are fixed as follows: $\mathrm{HMS}=1000, \mathrm{HMCR}=0.95$ and $\mathrm{PAR}=0.5$. The number of Sub-HM is 50. To show the performance of DHS algorithm, we compared the DHS algorithm to the GA algorithm [28] and EPABC algorithm [136]. All algorithms were coded in C++.net and run on Intel 2.8GHz PC with 1 GB memory. The two Orders were run for the 30 independent replications by three compared algorithms.

\subsection{Scheduling results and comparisons}

In this section, makespan, the mean of earliness and tardiness, and Pareto-based multi-objective criteria are considered. The size of re-processing problem in Order 1 is 12 jobs, 10 machines, and 44 operations while the corresponding values in Order 2 are 20, 12 and 91. Our purpose is to obtain high quality scheduling solutions for both criteria individually as well as together.

Table 6 - 1 The range of processing time of all jobs in Order 1

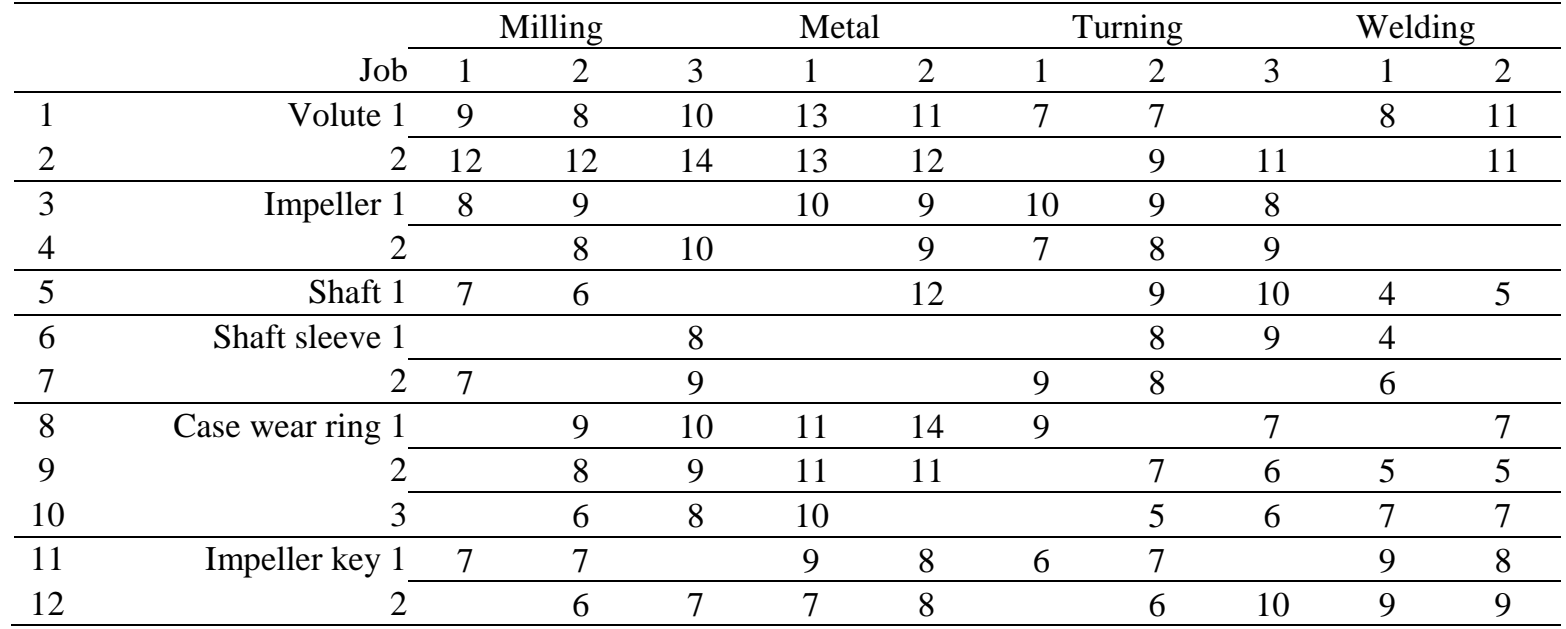

Table 6 - 2 The range of processing time of all jobs in Order 2

\begin{tabular}{|c|c|c|c|c|c|c|c|c|c|c|c|c|}
\hline \multirow{2}{*}{$\mathrm{Job}^{-}$} & \multicolumn{3}{|c|}{ Milling } & \multicolumn{2}{|c|}{ Metal } & \multicolumn{2}{|c|}{ Turning } & \multicolumn{3}{|c|}{ Welding } & \multicolumn{2}{|c|}{ Coating } \\
\hline & 1 & 2 & 3 & 1 & 2 & 1 & 2 & 3 & 1 & 2 & 1 & 2 \\
\hline Volute 1 & 8 & 6 & 8 & 10 & 8 & 6 & 5 & & 6 & 10 & 5 & 6 \\
\hline 2 & 11 & 10 & 12 & 12 & 11 & & 8 & 9 & & 9 & 6 & 4 \\
\hline 3 & 4 & 3 & & 9 & 6 & 7 & 6 & 7 & 10 & 8 & 4 & \\
\hline Impeller 1 & 7 & 8 & & 8 & 7 & 9 & 8 & 6 & & & 7 & 6 \\
\hline 2 & & 6 & 9 & & 7 & 5 & 7 & 6 & & & 5 & 6 \\
\hline 3 & 7 & & 6 & 6 & & 12 & & 11 & & & 3 & 5 \\
\hline
\end{tabular}




\begin{tabular}{|c|c|c|c|c|c|c|c|c|c|c|c|c|}
\hline Shaft 1 & 6 & 5 & & & 9 & & 6 & 8 & 2 & 4 & 3 & 3 \\
\hline 2 & & 7 & 7 & & 10 & 6 & 9 & & 3 & 3 & 3 & 3 \\
\hline \multirow[t]{2}{*}{ Shaft sleeve 1} & & 6 & & & & & 7 & 7 & 3 & & 4 & 5 \\
\hline & 6 & 5 & 7 & 5 & 6 & 7 & 5 & 7 & 5 & & 4 & 5 \\
\hline \multirow[t]{4}{*}{ Case wear ring 1} & & 8 & 7 & 8 & 12 & 6 & & 5 & & 5 & 2 & 2 \\
\hline & & 7 & 7 & 10 & 9 & & 6 & 4 & 3 & 4 & 5 & 6 \\
\hline & & 4 & 7 & 7 & & & 4 & 3 & 5 & 6 & 8 & 7 \\
\hline & & 5 & 3 & & 11 & 4 & & & 4 & 4 & 6 & 6 \\
\hline \multirow[t]{3}{*}{ Impeller wear ring 1} & 4 & 2 & & & & 8 & & 7 & & & 4 & 5 \\
\hline & 4 & + & 3 & & & 6 & 6 & 9 & & & 3 & 5 \\
\hline & & 4 & & & & & 7 & 6 & & & 4 & \\
\hline \multirow[t]{3}{*}{ Impeller key 1} & 6 & 5 & & 6 & 5 & 4 & 6 & & 8 & 6 & 4 & 7 \\
\hline & & 5 & 5 & 4 & 7 & & 5 & 8 & 7 & 6 & & 7 \\
\hline & & 4 & 5 & 6 & 7 & 6 & 7 & & 5 & 5 & & 5 \\
\hline
\end{tabular}

\subsubsection{Makespan criterion}

For Orders 1 and 2, the scheduling results by three algorithms are shown in Table 6 - 3. The first column of one algorithm is the minimum makespan value by this algorithm in 30 runs. The other three columns are the average value, maximum value and the value of standard deviation. It can be seen from Table 6 - 3 that DHS algorithm obtained the best min, avg, max and stdev values than GA and EPABC algorithms in 30 runs. The best makespan values of two Orders by DHS algorithm are 66 and 80 while the corresponding values by GA and EPABC algorithms are all 68 and 81 .

For the jobs and corresponding processing times in Table 6 - 2, the values of makespan with iteration times by three algorithms are shown in Fig. 6 - 4. It can be seen from Fig. 6 - 4 that DHS algorithm can obtain the best result of makespan when the iteration count is between 109 and 127. The DHS algorithm has a better convergence than GA algorithm. The EPABC algorithm has better convergence than DHS in the beginning. However, the EPABC algorithm falls into a local optimum around 100th iteration. Compared to EPABC algorithm, DHS has a superior ability to escape from local optima to generate better solutions eventually. 
To better guide the actual production in re-processing shop, Table 6 - 4 shows the completion time of all jobs in Order 1 and Order 2 when the values of makespan are 66 and 80 . The average processing time of all jobs in Order 1 is 52 while the corresponding value of Order 2 is 59.

Table 6 - 3 The results of Makespan criteria by three algorithms

\begin{tabular}{|c|c|c|c|c|c|c|c|c|c|c|c|c|}
\hline \multirow{2}{*}{ Order } & \multicolumn{4}{|l|}{ EPABC } & \multicolumn{4}{|l|}{ GA } & \multicolumn{4}{|l|}{ DHS } \\
\hline & $\min$ & ave & $\max$ & stdev & $\min$ & ave & $\max$ & stdev & $\min$ & ave & $\max$ & stdev \\
\hline 1 & 68 & 69 & 70 & 1.000 & 68 & 69.2 & 70 & 0.687 & 66 & 66.6 & 67 & 0.493 \\
\hline 2 & 81 & 82.5 & 85 & 1.269 & 81 & 82.6 & 84 & 0.966 & 80 & 80.4 & 81 & 0.5163 \\
\hline
\end{tabular}

\subsubsection{E/T scheduling results}

We discuss the E/T criterion for Order 1 and Order 2 in this section. The scheduling results of E/T by three algorithms are given in Table 6 - 5. The first column of one algorithm is the minimum E/T value by this algorithm in 30 runs. The other three columns are the average value, maximum value and the standard deviation value. It can be seen from Table 6 - 5 that DHS algorithm obtains the best min, avg, max and stdev values. The best E/T values of two Orders by DHS algorithm are 8 and 14 while the corresponding values by GA and EPABC algorithms are $(10,19)$ and $(10,18)$, respectively. The values of the best E/T of Order 1 and Order 2 are 8 and 14 and are not approximately zero (0). However, the results obtained by DHS algorithm are satisfactory in practice.

To better guide the actual production in re-processing shop, Fig. 6 - 5 shows the Gantt chart for Order 1 with the best E/T value (8). In Table 6 - 6 , the second column and the fifth column show the due date of all jobs in Order 2 while the third column and the sixth column show the completion time of all jobs with the best value of E/T (14). 


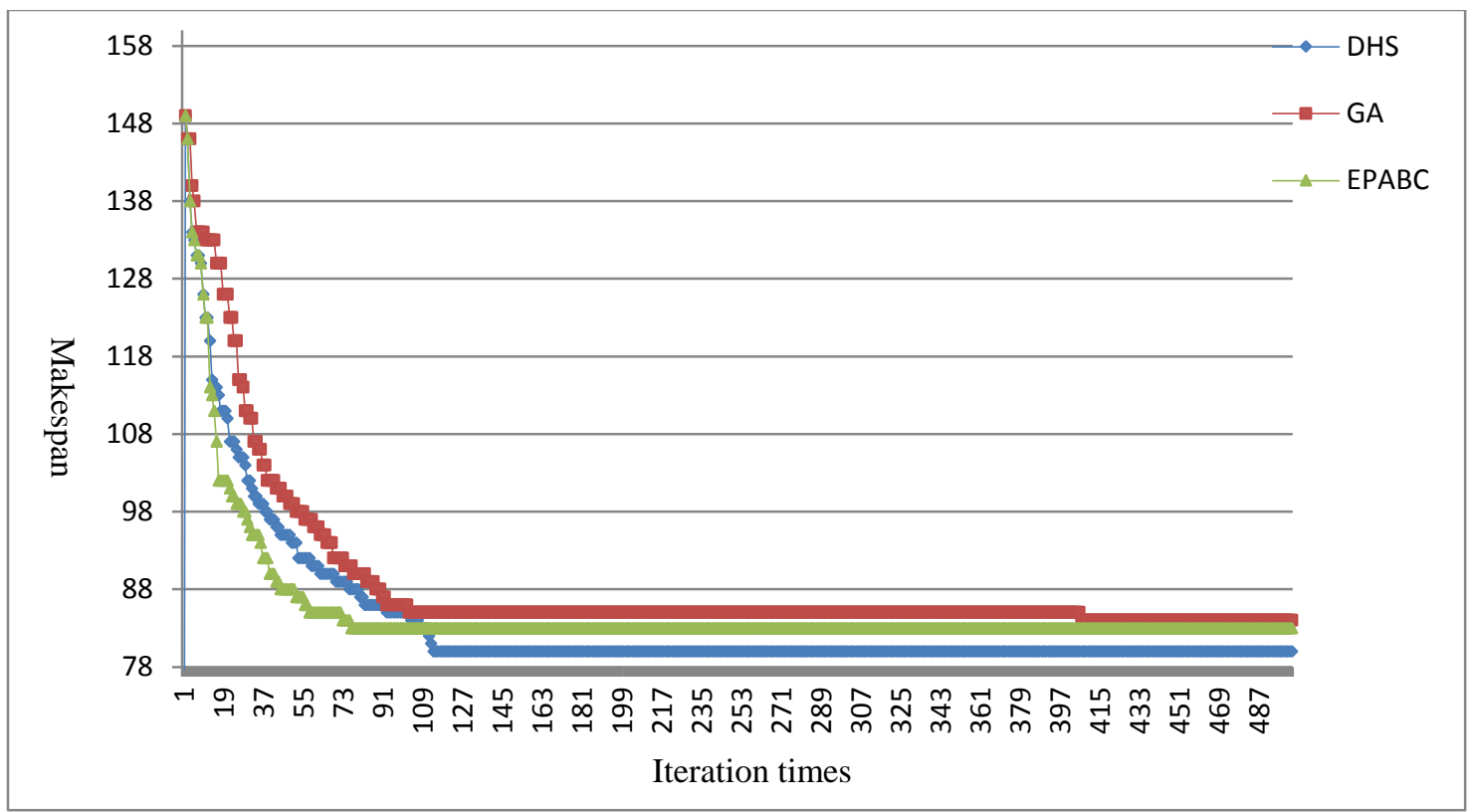

Fig. 6 - 4 The value of makespan with iteration

Table 6 - 4 The complete times of all jobs with best makespan in two Orders

\begin{tabular}{|c|c|c|c|}
\hline job & complete time & job & complete time \\
\hline Volute 1 & 34 & Volute 1 & 37 \\
\hline 2 & 47 & 2 & 76 \\
\hline Impeller 1 & 66 & 3 & 48 \\
\hline 2 & 66 & Impeller 1 & 67 \\
\hline Shaft 1 & 49 & 2 & 38 \\
\hline Shaft sleeve 1 & 53 & 3 & 42 \\
\hline 2 & 41 & Shaft 1 & 70 \\
\hline Case wear ring 1 & 54 & 2 & 80 \\
\hline 2 & 58 & Shaft sleeve 1 & 52 \\
\hline 3 & 65 & 2 & 75 \\
\hline Impeller key 1 & 64 & Case wear ring 1 & 80 \\
\hline 2 & 29 & 2 & 62 \\
\hline avg & 52 & 3 & 33 \\
\hline \multirow[t]{9}{*}{ Makespan } & 66 & 4 & 54 \\
\hline & & Impeller wear ring 1 & 67 \\
\hline & & 2 & 78 \\
\hline & & 3 & 56 \\
\hline & & Impeller key 1 & 60 \\
\hline & & 2 & 30 \\
\hline & & 3 & 72 \\
\hline & & avg & 59 \\
\hline & & Makespan & 80 \\
\hline
\end{tabular}


Table 6 - 5 The results of E/T criteria by three algorithms

\begin{tabular}{|c|c|c|c|c|c|c|c|c|c|c|c|c|}
\hline \multirow{2}{*}{ Order } & \multicolumn{4}{|l|}{ EPABC } & \multicolumn{4}{|l|}{ GA } & \multicolumn{4}{|l|}{ DHS } \\
\hline & $\min$ & ave & $\max$ & stdev & $\min$ & ave & $\max$ & stdev & $\min$ & ave & $\max$ & stdev \\
\hline 1 & 10 & 11 & 12 & 0.666 & 10 & 10.7 & 12 & 0.674 & 8 & 8.8 & 9 & 0.421 \\
\hline 2 & 18 & 20.3 & 22 & 1.251 & 19 & 20.2 & 21 & 0.788 & 14 & 14.4 & 15 & 0.516 \\
\hline
\end{tabular}

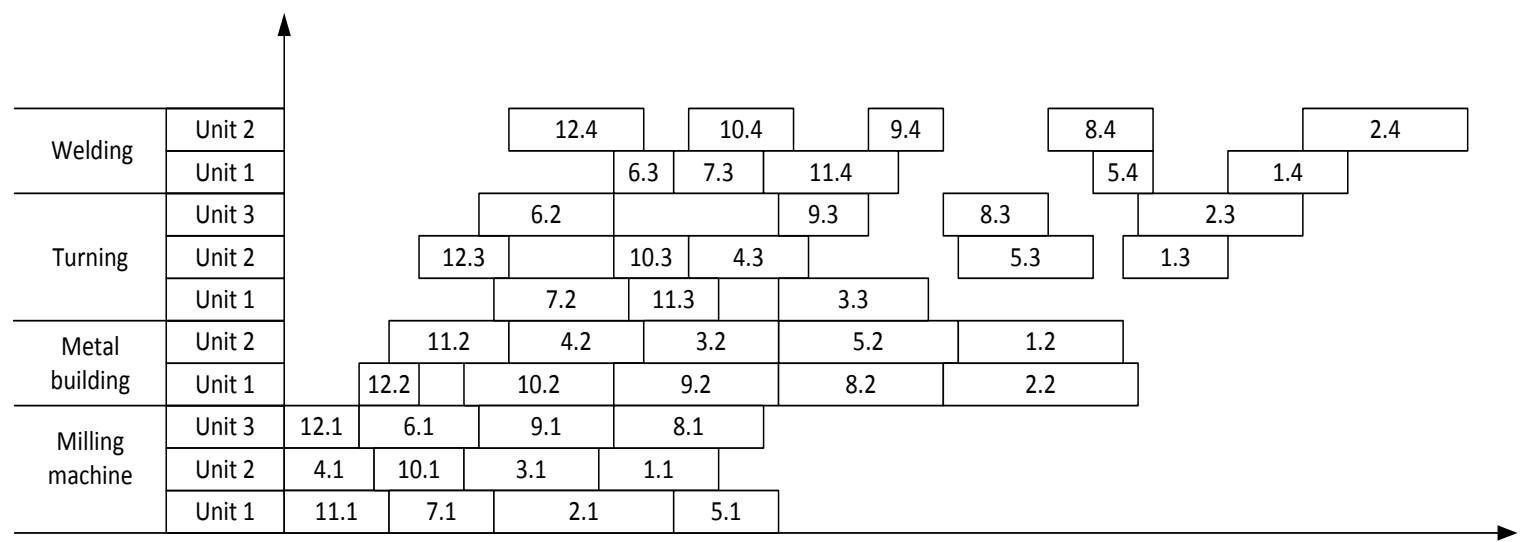

Fig. 6 - 5 The Gantt chart of Order 1 with E/T=8

Table 6 - 6 The completion times of all jobs with best E/T in Order 2

\begin{tabular}{|c|c|c|c|c|c|}
\hline Job & Due date & $\begin{array}{r}\text { Completion } \\
\text { time }\end{array}$ & Job & Due date & $\begin{array}{r}\text { Completion } \\
\text { time }\end{array}$ \\
\hline Volute 1 & 51 & 75 & Case wear ring 1 & 43 & 43 \\
\hline 2 & 66 & 90 & 2 & 43 & 63 \\
\hline 3 & 43 & 49 & 3 & 40 & 57 \\
\hline Impeller 1 & 40 & 41 & 4 & 43 & 33 \\
\hline 2 & 37 & 49 & Impeller wear ring 1 & 21 & 26 \\
\hline 3 & 40 & 56 & 2 & 21 & 22 \\
\hline Shaft 1 & 40 & 70 & 3 & 21 & 32 \\
\hline 2 & 45 & 79 & Impeller key 1 & 40 & 67 \\
\hline Shaft sleeve 1 & 30 & 25 & 2 & 43 & 63 \\
\hline 2 & 40 & 40 & 3 & 39 & 73 \\
\hline
\end{tabular}

\subsubsection{Pareto-based multi-objective scheduling}

For multi-objective optimization, the purposes are to obtain more non-dominated solutions that satisfy multiple objectives with good proximity and diversity with respect to the true Pareto front. Table 6 7 shows the Pareto-based non-dominated solutions obtained by three algorithms for Order 1 and Order 2. It can be seen from Table 6 - 7 that DHS algorithm obtains 6 solutions for Order 1 while the corresponding values by GA and EPABC are 4 and 6, respectively. There are 6 non-dominated solutions, $(77,8),(75,9),(74,10),(72,11),(68,18)$, and $(66,19)$ for Order 1 . Three algorithms can 
all obtain the non-dominated $(68,18)$. Only DHS algorithm can obtain 6 non-dominated solutions. For Order 2, the number of solutions by DHS algorithm is eight while the corresponding values by GA and $\mathrm{EPABC}$ are 7 and 6 . There are 9 non-dominated solutions, $(90,14),(87,15),(86,16),(85,17)$, $(84,18),(83,19),(82,32),(81,40)$, and $(80,43)$ for Order 2 . GA and EPABC algorithms have the same non-dominated solution $(81,40)$. DHS obtained 8 additional non-dominated solutions except the non-dominated solutions $(81,40)$. Hence, DHS algorithm finds more non-dominated solutions than GA and EPABC. For the merits of non-dominated solutions by three algorithms, Fig. 6 - 6 shows the non-dominated solutions by three algorithms for Order 2. It can be easily seen from Fig. 6 - 6 that the quality of non-dominated solutions obtained by DHS algorithm is better than those obtained by GA and EPABC algorithms. In addition, the best single objective solutions for two Orders are included in the non-dominated solutions obtained by DHS algorithm. For example, the best values of makespan for Order 1 and Order 2 are 66 and 80 while the corresponding values of E/T are 8 and 14. These four best single objective solutions correspond to the non-dominated solutions $(66,19),(80,43),(77,8)$ and $(90,14)$. Hence, the re-processing scheduling problem corresponding to Order 1 and Order 2 can be satisfactorily solved with respect to different single objective criterion and Pareto-based multiobjective criteria by DHS algorithm.

Table 6 - 7 Pareto-based non-dominated solutions by three algorithms

\begin{tabular}{|c|c|c|c|c|c|c|}
\hline \multirow[t]{2}{*}{ Order } & \multicolumn{2}{|l|}{ EPABC } & \multicolumn{2}{|l|}{ GA } & \multicolumn{2}{|l|}{ DHS } \\
\hline & Makespan & $\mathrm{E} / \mathrm{T}$ & Makepsan & $\mathrm{E} / \mathrm{T}$ & Makespan & $\mathrm{E} / \mathrm{T}$ \\
\hline \multirow[t]{6}{*}{1} & 80 & 10 & 79 & 10 & 77 & 8 \\
\hline & 75 & 11 & 75 & 12 & 75 & 9 \\
\hline & 74 & 12 & 72 & 13 & 74 & 10 \\
\hline & 70 & 14 & 68 & 18 & 72 & 11 \\
\hline & 69 & 17 & & & 68 & 18 \\
\hline & 68 & 18 & & & 66 & 19 \\
\hline \multirow[t]{8}{*}{2} & 90 & 18 & 93 & 17 & 90 & 14 \\
\hline & 89 & 19 & 92 & 19 & 87 & 15 \\
\hline & 86 & 20 & 91 & 22 & 86 & 16 \\
\hline & 85 & 22 & 85 & 24 & 85 & 17 \\
\hline & 83 & 33 & 84 & 31 & 84 & 18 \\
\hline & 81 & 40 & 83 & 37 & 83 & 19 \\
\hline & & & 81 & 40 & 82 & 32 \\
\hline & & & & & 80 & 43 \\
\hline
\end{tabular}




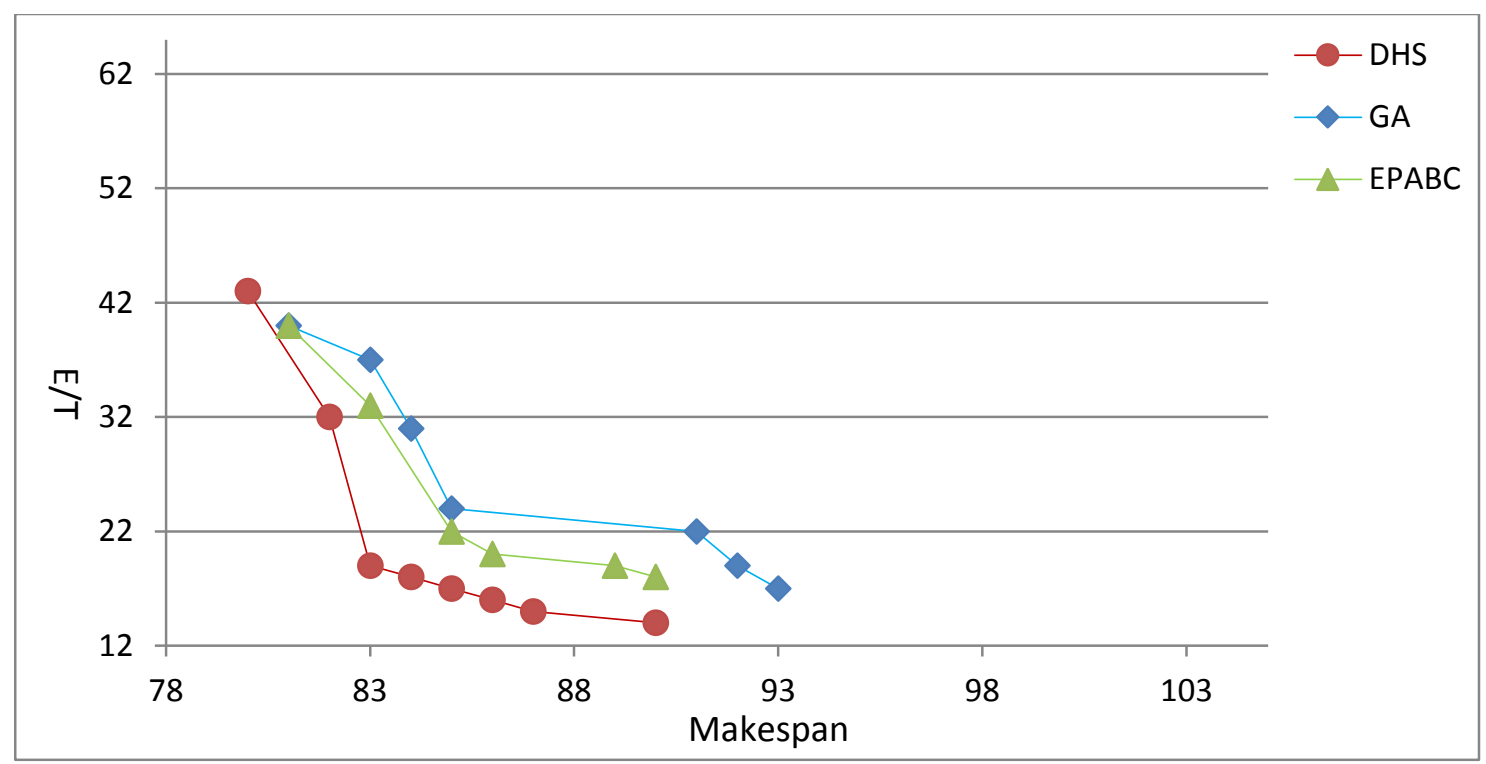

Fig. 6 - 6 Pareto-based non-dominate solutions

\subsubsection{Re-scheduling for processing time increasing}

In this section, we discuss the rescheduling problem for the processing time increasing in practice. According to scheduling results, the jobs will be processed in real re-processing shop floor. The processing time of one operation is estimated as a most probable processing time in advance. However, the processing time may be larger than the most probable processing time in some cases.

As shown in Fig. 6 - 8, the final scheduling result of makespan for Order 1 is 66 while the value of E/T in this schedule is 15 . When the jobs in Order 1 are processed in shop floor, the processing time of operation 2.2 (the second operation of job Volute 2) increases from 13 time units to 16 time units. If there is no rescheduling, the final Gantt chart after this change is shown in Fig. 6 - 7. The value of makespan is 68 and the increasing of $\mathrm{E} / \mathrm{T}$ criterion is 16.2. After completion of operation 2.2, the operations which can be re-scheduled are 1.4, 2.3, 2.4, 3.2, 3.3, 4.2, 4.3, 6.2, 6.3, 8.2, 8.3, 8.4, 9.3, 9.4, and 10.4. After rescheduling, the Gantt chart is shown in Fig. 6 - 10. The rescheduled operations are shown in dotted rectangles. The value of makespan after rescheduling is the same with that in Fig. 6 7. The increase of $\mathrm{E} / \mathrm{T}$ criterion reduces from 16.2 to 15.2. 


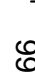

8
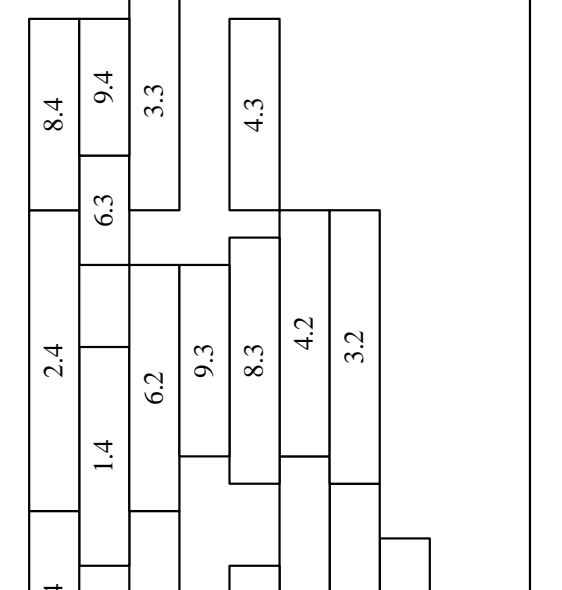

$\stackrel{+}{\circ}$

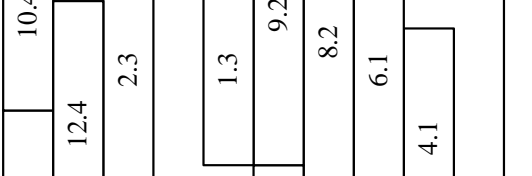

$\stackrel{+}{=}$

$=$

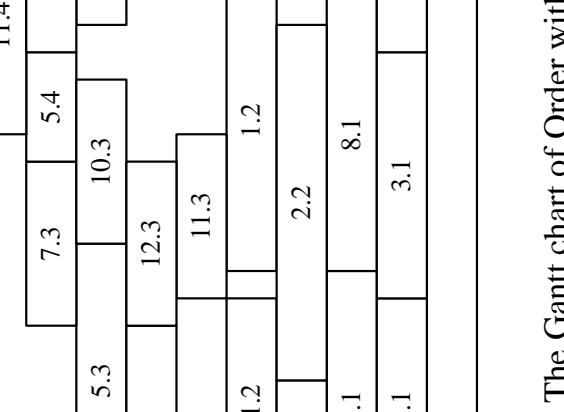

in

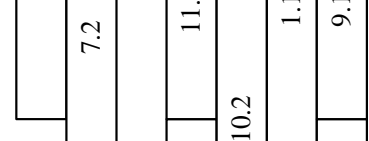

$\square$

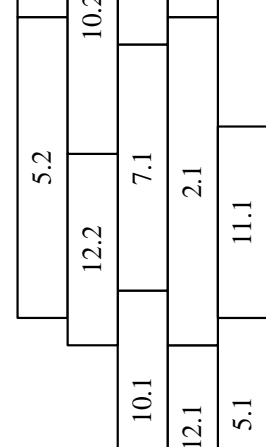

4

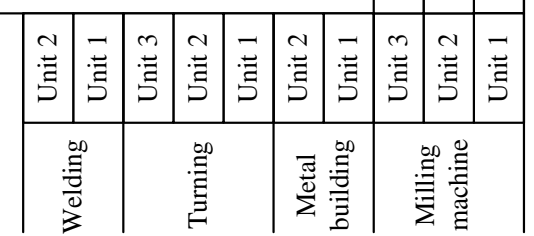

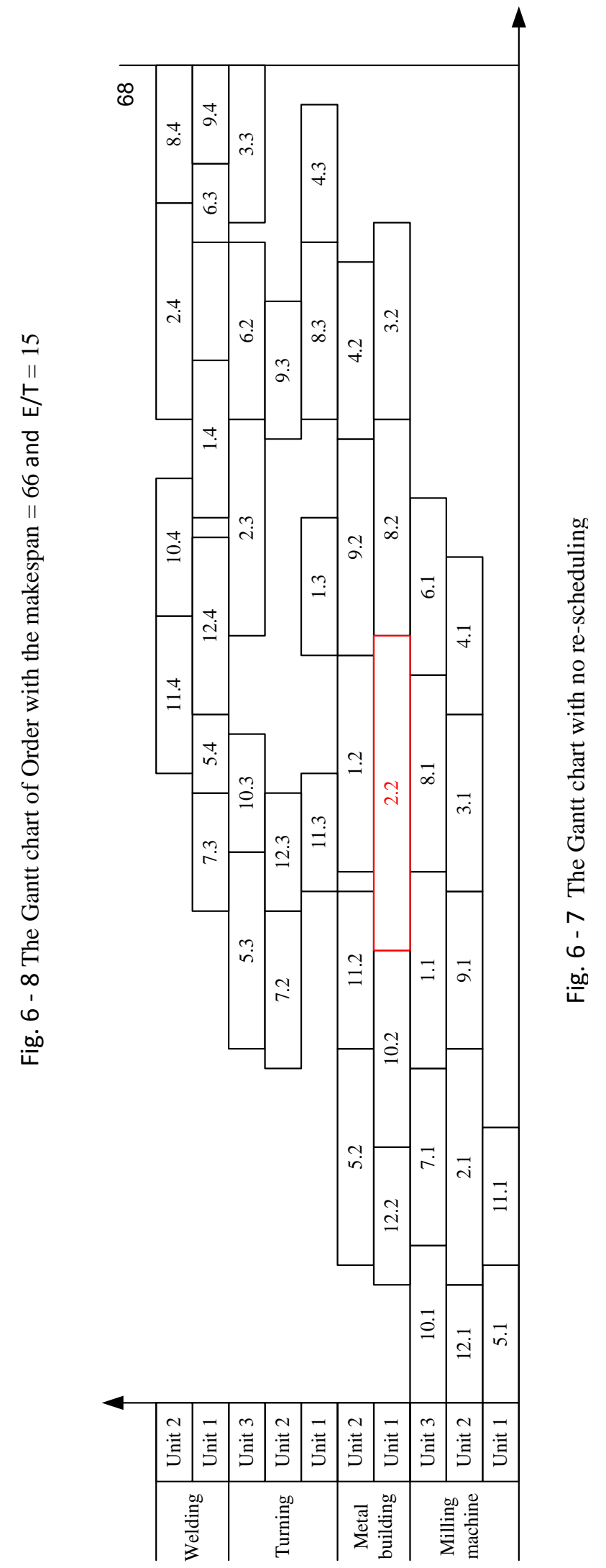




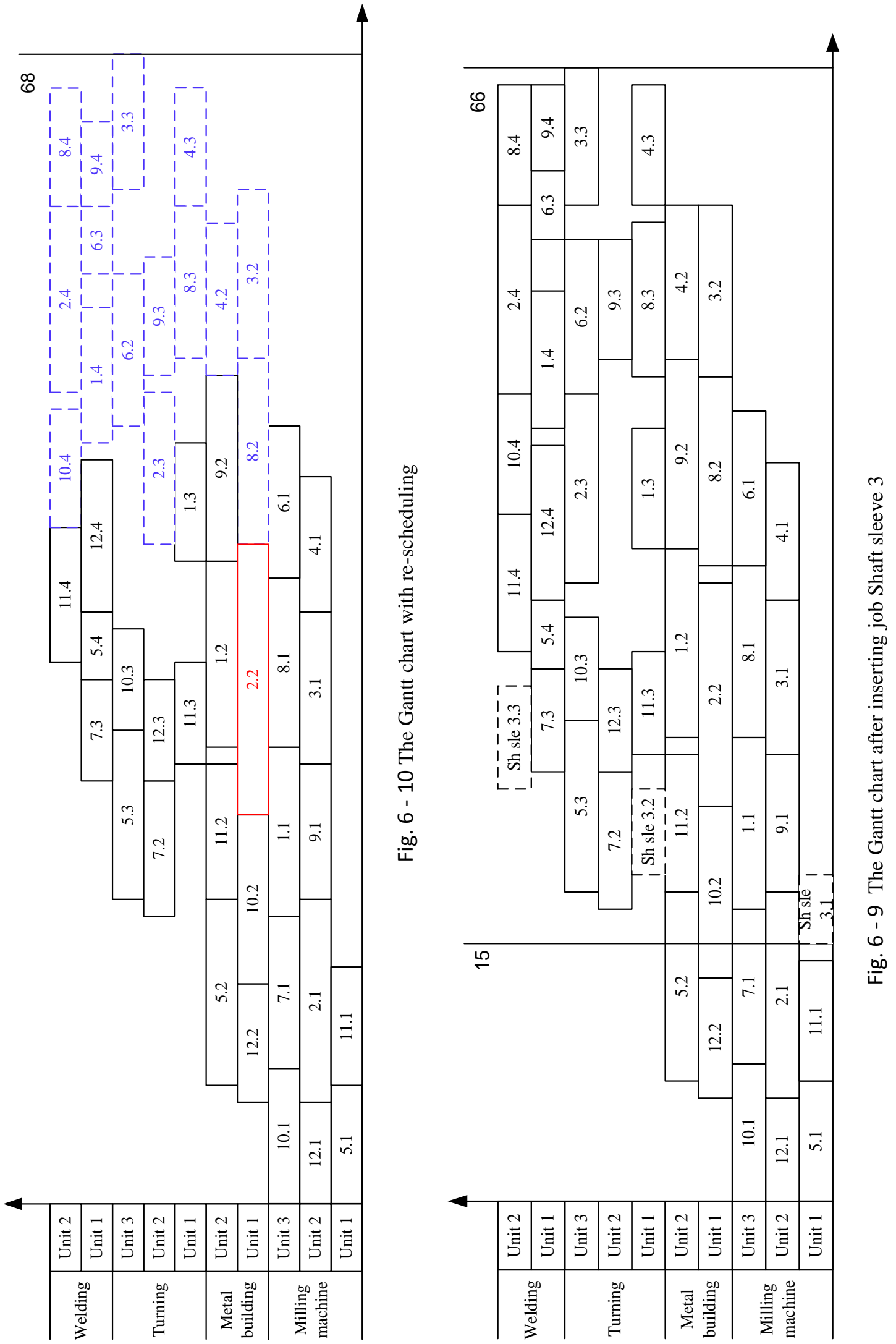




\subsubsection{Re-scheduling for a new job insertion}

In this section, we discuss the rescheduling in practice when a new job is inserted into the executing sequence. According to scheduling results for the Order 1, the value of makespan is 66 and E/T is 15 . The Gantt chart is shown in Fig. 6 - 8. When the jobs in Order 1 are re-processed in the shop, two new incoming jobs Shaft sleeve 3 and Shaft 2 are inserted. The operations and corresponding processing times are shown in Table 6 - 8. The Due dates of Shaft sleeve 3 and Shaft 2 are 15 and 26.

The job Shaft sleeve 3 is inserted into the scheduled sequence at 15 time units. After insertion, the Gantt chart is shown in Fig. 6 - 9 and the operations of Shaft sleeve 3 are in dotted rectangles. The value of makespan is not affected. The start time and completion time of Shaft sleeve 3 are 15 and 31 . Therefore, the tardiness time of Shaft sleeve 3 is 1 . The E/T values of all scheduled jobs are also unchanged. Hence, there is no need for rescheduling.

The Shaft 2 is inserted into the sequence at 32 time units. After insertion, the Gantt chart with no rescheduling is shown in Fig. 6 - 11. The operations of the Shaft 2 are also shown in dotted rectangles. The first operation of Shaft 2 is re-processed on unit 1 of Milling machine and the processing time is 4 time units. The third operation is re-processed on unit 1 of Turning machine. Both of the two operations are re-processed on the machines which have the minimum processing times among the selectable machines. The second and fourth operations have only one selectable machine. The value of makespan increases from 66 to 77. The start time and completion time of Shaft 2 are 32 and 77. The tardiness time of Shaft sleeve 3 is 45 . Hence, the operations which are not yet start at time 32 will be rescheduled. Fig. 6 - 12 shows the Gantt chart after rescheduling. There are nine operations changed their start times or processing machine, such as 2.2- 2.4, 8.2-8.4, 3.2-3.3 and 4.3. The completion times of job 3, 4, and 8 are increased from 66, 65, 65 to 72, 66, and 70, respectively. However, the completion time of Shaft 2 decreases from 77 to 63 . Hence, the E/T criterion is not affected obviously. The value of makespan reduces from 77 to 72 . The value of makespan is still larger than 66 , but it has been reduced from 77 to 72 by rescheduling.

Table 6 - 8 The operators and corresponding range of processing times of Shaft 2

\begin{tabular}{ccccccccccc}
\hline & \multicolumn{3}{c}{ Milling } & \multicolumn{2}{c}{ Metal } & \multicolumn{2}{c}{ Turning } & \multicolumn{2}{c}{ Welding } \\
Job & 1 & 2 & 3 & 1 & 2 & 1 & 2 & 3 & 1 & 2 \\
\hline Shaft sleeve & 3 & & 4 & & & 4 & 4 & 5 & 6 & 5 \\
3 & 4 & & 5 & & 5 & 6 & 6 & 7 & 6 \\
\hline \multirow{2}{*}{ Shaft 2 } & 3 & 4 & 5 & 5 & & 6 & 8 & & & 4 \\
& 4 & 5 & 6 & 7 & 7 & 10 & & \\
\hline
\end{tabular}




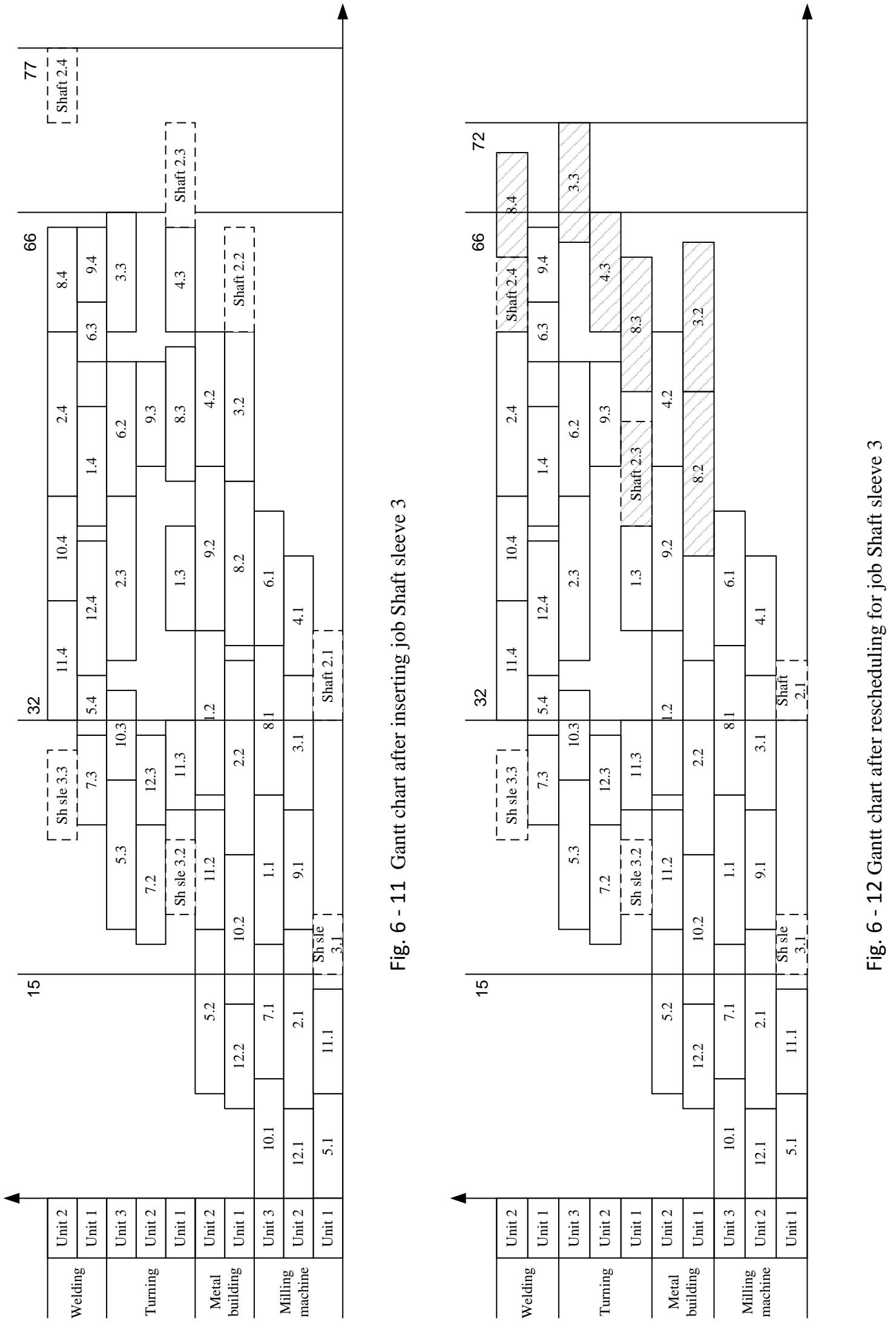




\subsection{Conclusions}

This Chapter investigated the re-processing scheduling and rescheduling problem in remanufacturing system. The scheduling and rescheduling problem of re-processing were modeled. Considering the processing time of each job is unpredictable, the processing time was estimated as a most probable value by experience. Discrete harmony search algorithm and local search method were employed for this problem. Rescheduling strategy was proposed for processing time increasing and new job insertion in practice. Makespan, mean of earliness and tardiness objectives were considered. As a case study, we solved two instances from real-life remanufacturing engineering and described the process in detail. The results demonstrate DHS algorithm can effectively solve scheduling and rescheduling problems in practice. 


\section{Chapter 7}

\section{FDHS Algorithm for FJSSP with Fuzzy Processing Time}

\subsection{Introduction}

In this Chapter, we propose an effective fuzzy discrete harmony search (FDHS) algorithm to solve the FJSSP with fuzzy processing time. The objective is to minimize the maximum fuzzy completion time. A simple and effective heuristic, namely MinEnd, is proposed to initialize population. To test performance, MinEnd is compared to several existing heuristics. FDHS is tested using existing benchmark cases and real instances from remanufacturing. The experimental results and comparisons demonstrate the performance of FDHS.

The rest of this Chapter is organized as follows: Section 7.2 briefly describes the objective of FJSSP with fuzzy processing time constraint. The MinEnd heuristic is presented in Section 7.3. Section 7.4 presents the method for improvising new harmony in FDHS. The framework of the proposed FDHS algorithm is given in Section 7.5. Section 7.6 is for experimental results and comparisons. We conclude this Chapter in Section 7.7. 


\subsection{FJSSP with fuzzy processing time}

The FJSSP with fuzzy processing time means that the operation processing time is not an exact value and the processing time is represented with triangular fuzzy number (TFN) as follows:

$$
t_{i, j, k}=\left(t_{i, j, k}^{1}, t_{i, j, k}^{2}, t_{i, j, k}^{3}\right)
$$

where $t_{i, j, k}^{1}, t_{i, j, k}^{2}$ and $t_{i, j, k}^{3}$ are three probable processing times of operation $\boldsymbol{O}_{i, j}$ on machine $\boldsymbol{M}_{k}$. The fuzzy completion time of operation $O_{i, j}$ is also a TFN as follows:

$$
C_{i, j}=\left(C_{i, j}^{1}, C_{i, j}^{2}, C_{i, j}^{3}\right)
$$

where $C_{i, j}^{1}, C_{i, j}^{2}$ and $C_{i, j}^{3}$ are three probable completion times of operation $O_{i, j}$.

The objective is to minimize maximum fuzzy completion time. The maximum fuzzy completion time denoted by $C_{M}$ can be calculated by the following formula.

$$
\text { Min } C_{M}=\max _{1 \leq i \leq n}\left\{C_{i}\right\}
$$

where $C_{i}$ is the fuzzy completion time of job $i$.

\subsection{Proposed initializing rule}

For meta-heuristics, the quality of initial population often affects the speed of convergence. Therefore, it is a critical step to generate a high quality initial population. For machine assignment, there are random rule, local minimal processing time (LS) rule and global minimal processing time rule (GS). For operation sequencing, random rule, most work remaining rule (MReW) and most number of operations remaining $(\mathrm{MReO})$ rule were proposed in literature [16].

In this Chapter, we proposed a new heuristic, named MinEnd heuristic, for initializing population. In this heuristic, the operation sequence is decided randomly. The processing machine for each operation is assigned based on the operation sequence. The steps of this heuristic are shown as follows: Step1: All operations of all jobs are shuffled randomly to obtain an operation sequence (OS).

Step2: Repair OS, make sure the operations of the same job can satisfy the processing precedence. Step3: For each operation $O_{i, j}$, evaluate the fuzzy completion time on each selectable machine.

Step4: The machine with minimum fuzzy completion time is selected for processing operation $O_{i, j}$. The minimum computational complexity to obtain a random operation sequence is $O\left(\sum_{i=1}^{n} q_{i}\right)$. The complexity to repair operation sequence is $O\left(\sum_{i=1}^{n} q_{i} * \log _{2} \sum_{i=1}^{n} q_{i}\right)$. The complexity to select machine for all operatons is $O\left(\sum_{i=1}^{n} q_{i}\right)$. Hence, the computational complexity of the MinEnd heuristic 
is $O\left(\sum_{i=1}^{n} q_{i} *\left(2+\log _{2} \sum_{i=1}^{n} q_{i}\right)\right)$. To describe this rule clearly, we take the problem shown in Table 7 - 1 to represent the heuristic rule in detail. In Table 7 - 1, there are three jobs, three machines and ten operations. First, we generate an operation sequence randomly. The operation sequence is as $\mathrm{OS}=\{(3,2),(2,1),(1,3),(1,1),(2,2),(2,4),(3,3),(2,3),(3,1),(1,2)\}$. In step2, the operations sequence is repaired to satisfy the processing precedence of the same job's operations. The operation sequence is changed as $O S=\{(3,1),(2,1),(1,1),(1,2),(2,2),(2,3),(3,2),(2,4),(3,3),(1,3)\}$. Step3 is to select processing machine for each operation. For the first operation $(3,1)$, selectable machines are $M_{1}$ and $\boldsymbol{M}_{2}$. The fuzzy completion time on machine $M_{1}$ is $(2,4,8)$ while the fuzzy completion time on $\boldsymbol{M}_{2}$ is $(4,5,7)$. Based on the fuzzy TFNs ranking criteria, $(4,5,7)$ is larger than $(2,4,8)$. Hence, $M_{1}$ is selected for operation $(3,1)$. The available time of $M_{1}$ for next operation is $(2,4,8)$. The second operation is $(2,1)$. The fuzzy completion on selectable machine $\boldsymbol{M}_{2}, \boldsymbol{M}_{3}$ and $\boldsymbol{M}_{4}$ are $(5,7,9),(1,2$, 5) and (1, 2, 3), respectively. Machine $\boldsymbol{M}_{4}$ is selected and the available time becomes $(1,2,3)$. For third operation(1,1), the selectable machines are $\boldsymbol{M}_{2}$ and $\boldsymbol{M}_{4}$. The fuzzy completion time on $\boldsymbol{M}_{2}$ is $(5,6,7)$. Because the available time of $M_{4}$ is $(1,2,3)$, the fuzzy completion time on $\boldsymbol{M}_{4}$ is the addition of $(1,2,3)$ and $(3,5,6)$. Based on the TFNs addition operation, the fuzzy completion time is $(4,7,9)$. Based on the TFNs ranking criteria, $(4,7,9)$ is larger than $(5,6,7)$. So, $M_{2}$ is selected for operation $(1,1)$ and the available time becomes $(5,6,7)$. All remaining operations can be assigned to one processing machine in the same way. The final operation order on each machine are $M_{1}=O_{3,1}, O_{1,2}, O_{2,4}, M_{2}=O_{1,1}, O_{3,3}, M_{3}=O_{3,2}, O_{1,3}$ and $M_{4}=O_{2,1}, O_{2,2}, O_{2,3}$. The Gantt chart of fuzzy completion time is shown in Fig. 7 - 1.

Table 7 - 1 An example of FJSSP fuzzy processing time

\begin{tabular}{cccccc}
\hline \multirow{2}{*}{ Job } & Operation & \multicolumn{4}{c}{ Machine } \\
\cline { 3 - 6 } & & $M_{1}$ & $M_{2}$ & $M_{3}$ & $M_{4}$ \\
\hline \multirow{2}{*}{1} & $O_{11}$ & $(1,2,3)$ & $(6,8,10)$ & $(5,9,11)$ & $(9,5,10,14)$ \\
& $O_{12}$ & $(1,3,4)$ & - & $(4,6,8)$ & - \\
& $O_{13}$ & - & $(5,7,9)$ & $(1,2,5)$ & $(1,2,3)$ \\
2 & $O_{21}$ & $(5,7,9)$ & $(4,5,7)$ & $(3,5,6)$ & $(1,2,3)$ \\
& $O_{22}$ & $(7,8,11)$ & - & - & $(1,3,4)$ \\
& $O_{23}$ & $(7,9,10)$ & - & $(5,6,9)$ & - \\
& $O_{24}$ & $(2,4,8)$ & - & $(4,5,7)$ & - \\
& $O_{31}$ & $(6,8,12)$ & $(5,8,10)$ & $(6,7,10)$ & $(4,7,9)$ \\
& $O_{32}$ & $(8,9,12)$ & $(3,4,5)$ & - & - \\
\hline
\end{tabular}




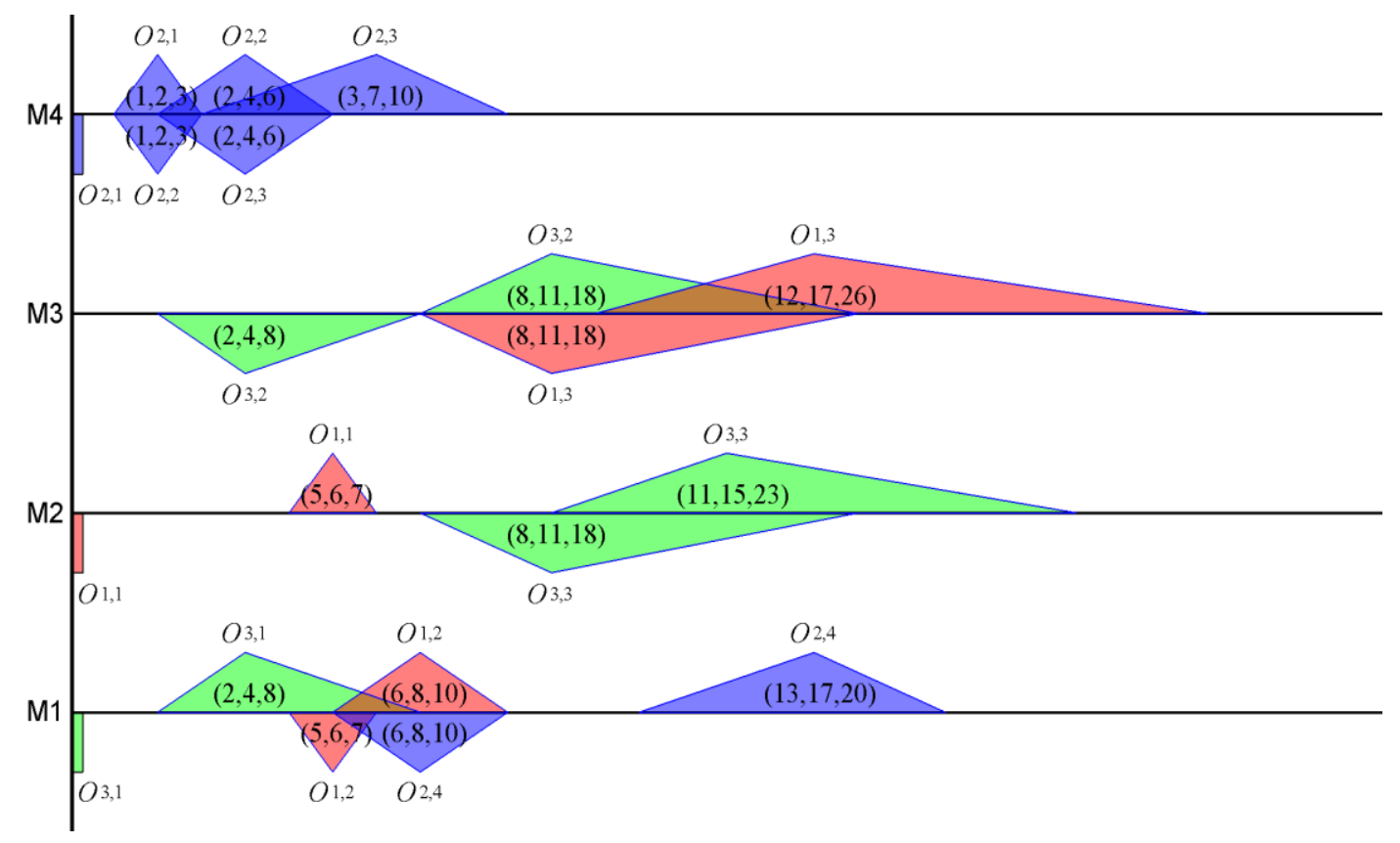

Fig. 7 - 1 Fuzzy Gantt chart by proposed heuristic rule

\subsection{Improvising new harmony}

In the basic harmony search algorithm, a new harmony is generated based on harmony memory or randomly. In this Chapter, we generate two new harmonies based on two harmonies in harmony memory at the same time. The steps are shown as follows:

Procedure: Improvising two new harmonies $x_{n e w}^{1}$ and $x_{\text {new }}^{2}$

Step1: For each operation $j, 1 \leq j \leq$ Toper.Toper is the total number of operations.

Step2: Generate one random number $R 1$. If $R 1<H M C R$, go to Step3; else, go to Step6. $x_{a}$ and $x_{b}$ are two harmonies in harmony memory.

Step3: If there is no value in $j^{\text {th }}$ position of $x_{\text {new }}^{1}, x_{\text {new }}^{1}(j)=x_{a}(j)$; else, find the first position $(p)$ place with no value in $x_{\text {new }}^{1}$ and set $x_{\text {new }}^{1}(p)=x_{a}(j)$. If there is no value in $j^{\text {th }}$ position of $x_{\text {new }}^{2}$, $x_{\text {new }}^{2}(j)=x_{b}(j)$; else, find the first position $\left(p^{\prime}\right)$ with no value in $x_{\text {new }}^{2}$ and set $x_{\text {new }}^{2}\left(p^{\prime}\right)=x_{b}(j)$.

Step4: Generate one random number $R 2$. If $R 2<P A R$, go to Step5; else, go to Step7.

Step5: Search the place $(p 1)$ of $x_{\text {new }}^{1}(j)$ in $x_{b}$, exchange $x_{\text {new }}^{1}(j)$ and $x_{\text {new }}^{1}(p 1)$; search the place $(p 2)$ of $x_{\text {new }}^{2}(j)$ in $x_{a}$, exchange $x_{\text {new }}^{2}(j)$ and $x_{\text {new }}^{2}(p 2)$. 
Step6: If the processing machine for $x_{\text {new }}^{1}(j)$ has the minimum processing time, random select a candidate machine for $x_{\text {new }}^{1}(j)$. If the processing machine for $x_{\text {new }}^{2}(j)$ has not the minimum processing time, select the machine with minimum processing time for $x_{\text {new }}^{2}(j)$.

Step7: Repair $x_{n e w}^{1}$ and $x_{n e w}^{2}$ to make sure that the operations of the same job can satisfy the processing precedence.

\begin{tabular}{|c|c|c|c|c|c|c|c|c|c|c|}
\hline \multirow[b]{2}{*}{$x_{a}$} & & \multirow{2}{*}{$\begin{array}{r}\mathrm{R} 2<\mathrm{PAR} \\
(1,1,4) \\
\end{array}$} & & $\mathrm{R} 2<\mathrm{PAR}$ & & & & $\mathrm{R} 2<\mathrm{PAR}$ & \\
\hline & $(2,1,3)$ & $(3,1,1)$ & & $(1,2,3)$ & $(3,2,2)$ & $(2,2,1)$ & $(2,3,4)$ & $(1,3,1)$ & $(3,3,2)$ & $(2,4,3)$ \\
\hline $\mathrm{x}_{\mathrm{b}}$ & $(1,1,2)$ & $(2,1,3)$ & $(2,2,4)$ & $(3,1,2)$ & $(2,3,1)$ & $(3,2,1)$ & $(1,2,3)$ & $(1,3,2)$ & $(2,4,4)$ & $(3,3,1)$ \\
\hline position & 1 & 2 & 3 & 4 & 5 & 6 & 7 & 8 & 9 & 10 \\
\hline \multirow{3}{*}{$\underset{\mathrm{x}_{\text {new }}^{1}}{\longrightarrow}$} & \multicolumn{10}{|l|}{$\mathrm{J}=1,2$} \\
\hline & $(2,1,3)$ & $(3,1,1)$ & & & & & & & & \\
\hline & $(1,1,2)$ & $(2,1,3)$ & & & & & & & & \\
\hline$\rightarrow$ & \multicolumn{3}{|l|}{$\mathrm{J}=3$} & & & & & & & \\
\hline$x^{1}{ }_{\text {new }}$ & $(1,1,4)$ & $(3,1,1)$ & $(2,1,3)$ & & & & & & & \\
\hline $\mathrm{x}_{\text {new }}^{2}$ & $(1,1,2)$ & $(2,1,3)$ & & & & $(2,2,4)$ & & & & \\
\hline \multicolumn{11}{|c|}{$\begin{array}{l}\longrightarrow \mathrm{J}=\cdots \\
\mathrm{J}=8\end{array}$} \\
\hline $\mathrm{x}_{\text {new }}^{1}$ & $(1,1,4)$ & $(3,1,1)$ & $(2,1,3)$ & $(1,2,3)$ & $(2,2,1)$ & $(3,2,2)$ & $(2,3,4)$ & $(1,3,3)$ & & \\
\hline $\mathrm{x}_{\text {new }}^{2}$ & $(1,1,2)$ & $(2,1,3)$ & $(3,2,1)$ & $(3,1,2)$ & $(1,2,3)$ & $(2,2,4)$ & $(2,3,1)$ & $(1,3,2)$ & & \\
\hline \multicolumn{11}{|c|}{$\begin{array}{l}\longrightarrow \mathrm{J}=\cdots \\
\mathrm{J}=10\end{array}$} \\
\hline $\mathrm{x}_{\text {new }}^{1}$ & $(1,1,4)$ & $(3,1,1)$ & $(2,1,3)$ & $(1,2,3)$ & $(2,2,1)$ & $(3,2,2)$ & $(2,3,4)$ & $(1,3,3)$ & $(2,4,3)$ & $(3,3,2)$ \\
\hline $\mathrm{x}^{2}{ }_{\text {new }}$ & $(1,1,2)$ & $(2,1,3)$ & $(3,2,1)$ & $(3,1,2)$ & $(1,2,3)$ & $(2,2,4)$ & $(2,3,1)$ & $(1,3,2)$ & $(3,3,1)$ & $(2,4,4)$ \\
\hline \multicolumn{11}{|c|}{ Repair $x_{\text {new }}^{2}$} \\
\hline & $(1,1,2)$ & $(2,1,3)$ & $(3,1,2)$ & $(3,2,1)$ & $(1,2,3)$ & $(2,2,4)$ & $(2,3,1)$ & $(1,3,2)$ & $(3,3,1)$ & $(2,4,4)$ \\
\hline
\end{tabular}

Fig. 7 - 2 Improvising two new harmonies

To represent the steps for improvising new harmony in detail, an example is shown in Fig. 7 - 2. There are 3 jobs, 4 machines and 10 operations in this example. When $j=1$ and $j=2$, the random $R 1$ is less than HMCR. The operation in ${ }^{x_{\text {new }}^{1}}(j)$ equals that in $x_{a}(j)$ and the operation in $x_{\text {new }}^{2}(j)$ equals that in $x_{b}(j)$. When ${ }^{j=3}$, the random number $R 2$ is less than $P A R$. The operation in $x_{\text {new }}^{1}(3)$ equals that $x_{a}(3)$ and the operation in ${ }^{x_{\text {new }}^{2}}(3)$ equals that in $x_{b}(3)$. The position of operation $(p 1)$ of $x_{\text {new }}^{1}(3)$ in $x_{b}$ is 1 . Hence, the values in $x_{\text {new }}^{1}(1)$ and $x_{\text {new }}^{1}(3)$ are changed. The position of operation ( ${ }^{2}$ ) of $x_{\text {new }}^{2}(3)$ in $x_{a}$ is 6 . Hence, the values of $x_{\text {new }}^{2}(3)$ is put into $x_{\text {new }}^{2}(6)$ and $x_{\text {new }}^{2}(3)$ remains null. The $x_{\text {new }}^{1}$ and $x_{\text {new }}^{2}$ can be obtained in the same way until $j=8$. When $j=8$, the random $R 1$ is larger than $H M C R$. The processing machine of operation in ${ }^{x_{\text {new }}^{1}}(8)$ is changed from machine ${ }^{M_{1}}$ to $\boldsymbol{M}_{3}$. After considering all operations, $x_{n e w}^{1}$ and $x_{\text {new }}^{2}$ an be obtained. However, the first and second operations of job 3 in $x_{\text {new }}^{2}$ do not satisfy the processing priority. After changing the positions of these two operations, the two new harmonies $x_{\text {new }}^{1}$ and $x_{\text {new }}^{2}$ are obtained finally. 


\subsection{Procedure of Proposed FDHS}

Based on the above design, the FDHS in this Chapter employs random rule, global minimal processing time rule and the MinEnd heuristic proposed in Section 4.2 to initialize harmony memory or population. The computational procedure of proposed FDHS for FJSSP with fuzzy processing time can be described as follows:

Procedure: Proposed FDHS

Step1: Set parameters, HMS, HMCR, PAR.

Step2: Initialize harmony memory $H M$ using MinEnd heuristic, global minimal processing time rule and random rule.

Step3: Evaluate fuzzy completion time of all harmony and update the best fuzzy completion time and the best harmony.

Step4: Improvising new harmonies based on the method proposed in Section 4.3.

Step5: Evaluate fuzzy completion times of all new harmonies and update the best fuzzy completion time and the best harmony.

If the number of iterations is less than the maximum generation, go to Step 4; else, output the best solution.

\subsection{Experiment evaluation and comparisons}

\subsubsection{Experiment setup}

To evaluate the performance of proposed FDHS algorithm, experimental evaluation and comparisons are conducted. Two sets of instances are studied in this Chapter. Set one includes five FJSSP cases with fuzzy processing time [82,147]. The sizes are ranging from 10-jobs, 10-machines and 40-operations to 15-jobs, 10-machines and 80-operations. Set two includes eight instances from remanufacturing engineering. The sizes are from 5-jobs, 4-machines and 23-operations to 20-jobs, 15machines and 355-operations. The proposed FDHS algorithm is coded in C++ and run on Intel 2.40 $\mathrm{GHz}$ PC with $2 \mathrm{~GB}$ memory. The parameters are set based on our parameter tuning simulations as follows: $\mathrm{HMS}=150, \mathrm{HMCR}=0.95$ and $\mathrm{PAR}=0.5$. For instances in set one and the first four instances in Set two, the generation is set to 1000 to meet the same number of evolutions as compared algorithms. For the last four instances in Set two, the generation is set to 4000 because the sizes of these four instances are larger than other instances. All experiments are carried out with 30 replications.

\subsubsection{Performance of Proposed MinEnd Heuristic}

To test the performance of the proposed MinEnd heuristic, we compare the average fuzzy completion time results of five cases in set one by six heuristic rules, MinEnd, GS, LS, MReW, MReO 
and random. The results are shown in Table 7 - 2. Compared to random rule, MReW and MReO rules cannot improve the average fuzzy completion times. GS rule can find better average fuzzy completion times than LS, MReW, MReO and random rules for case 2 and case 3. LS rule can get better fuzzy completion times than GS, MReW, MReO and random rules for case 1, case 4 and case 5. GS and LS have better performance than $\mathrm{MReW}, \mathrm{MReO}$ and random rules. Compared to GS and LS rules, MinEnd heuristic can obtain better average fuzzy completion time results for all five cases and the improvement is obvious. The CPU times of MinEnd heuristic and all the compared heuristics are very limited and can be ignored. Hence, the proposed MinEnd heuristic rule is effective for initializing harmony memory.

Table 7 - 2 Maximum fuzzy completion time results by six heuristic rules

\begin{tabular}{|c|c|c|c|c|c|c|c|c|c|}
\hline \multirow{2}{*}{$\begin{array}{l}\text { Instance } \\
\text { Case1 }\end{array}$} & \multirow{2}{*}{$\begin{array}{l}\text { Algorithm } \\
\text { MinEnd }\end{array}$} & \multicolumn{3}{|c|}{$\begin{array}{c}\text { Ave Fuzzy } \\
\text { completion time }\end{array}$} & \multirow{2}{*}{$\begin{array}{l}\text { Instance } \\
\text { Case4 }\end{array}$} & \multirow{2}{*}{$\begin{array}{l}\text { Algorithm } \\
\text { MinEnd }\end{array}$} & \multicolumn{3}{|c|}{$\begin{array}{c}\text { Ave Fuzzy } \\
\text { completion time }\end{array}$} \\
\hline & & 45.6 & 61.9 & 81.0 & & & 34.9 & 51.9 & 72.2 \\
\hline & GS & 52.0 & 74.3 & 96.2 & & GS & 55.9 & 79.4 & 105.6 \\
\hline & LS & 49.4 & 71.2 & 90.9 & & LS & 54.7 & 78.1 & 103.1 \\
\hline & MReW & 59.3 & 88.9 & 117.5 & & MReW & 64.4 & 87.7 & 113.4 \\
\hline & $\mathrm{MReO}$ & 59.7 & 88.8 & 117.0 & & $\mathrm{MReO}$ & 61.8 & 85.6 & 110.6 \\
\hline & Random & 59.2 & 88.0 & 116.2 & & Random & 71.4 & 98.5 & 127.0 \\
\hline \multirow[t]{6}{*}{ Case2 } & MinEnd & 45.6 & 61.9 & 81.0 & Case 5 & MinEnd & 53.7 & 78.5 & 108.2 \\
\hline & GS & 80.7 & 108.3 & 138.5 & & GS & 80.9 & 113.5 & 150.4 \\
\hline & LS & 81.5 & 109.9 & 140.3 & & LS & 81.2 & 111.3 & 146.8 \\
\hline & MReW & 98.4 & 132.6 & 168.8 & & MReW & 92.1 & 129.1 & 170.8 \\
\hline & $\mathrm{MReO}$ & 102.9 & 139.3 & 177.9 & & $\mathrm{MReO}$ & 95.5 & 134.1 & 177.5 \\
\hline & Random & 99.0 & 134.4 & 171.6 & & Random & 94.4 & 132.7 & 174.7 \\
\hline \multirow[t]{6}{*}{ Case3 } & MinEnd & 46.1 & 65.9 & 85.6 & & & & & \\
\hline & GS & 72.1 & 97.7 & 125.1 & & & & & \\
\hline & LS & 77.3 & 105.6 & 135.7 & & & & & \\
\hline & MReW & 88.3 & 117.9 & 151.5 & & & & & \\
\hline & $\mathrm{MReO}$ & 83.3 & 111.7 & 143.7 & & & & & \\
\hline & Random & 85.5 & 114.1 & 146.9 & & & & & \\
\hline
\end{tabular}

\subsubsection{Comparison and discussion}

For the five cases in set one, the proposed FDHS algorithm is compared to six existing algorithms: EDA [77], HABC [137], DIGA [147], CGA [55], PEGA [16] and PSO+SA [23]. The results of average fuzzy completion times, best fuzzy completion times and the worst fuzzy completion times are shown in Table 7 - 3.

It can be seen from Table 7 - 3 that the proposed FDHS algorithm performs better than all compared algorithms. For five cases, FDHS algorithm can obtain better average fuzzy completion times and best fuzzy completion times than those by six compared algorithms. In 30 runs, FDHS can find minimum 
worst fuzzy completion time for case 2, case 3 and case 4. EDA algorithm finds minimum worst fuzzy completion time for case 1 and case 5 . The best solution obtained by FDHS for case 1 is $(17,29,39)$ and the fuzzy Gantt chart is illustrated in Fig. 7 - 3.

The average CPU time of all compared algorithms are listed in Table 7 - 4. Compared to the six algorithms, FDHS has the minimum average CPU time. For case 1 to case 4, the average time of FDHS is less than 1.5 seconds. For the largest case, case 5, the average CPU time is just 2.65 seconds. Although the CPU frequencies are different among seven algorithms, we can conclude that the proposed FDHS algorithm is the most efficient one.

To further test the performance of proposed FDHS algorithm, the eight instances in set two are solved. The average, best and worst results by FDHS algorithm and MinEnd heuristic are shown in Table 7 5. It can be seen from Table 7 - 5 that FDHS can improve MinEnd obviously for all instances. FDHS can improve the average fuzzy completion time results by MinEnd more than $30 \%$ for all instances. Fig. 7 - 4 shows the convergence curve of three ranking criteria results by FDHS. For the same value of the first ranking criterion, the values of the second and the third ranking criteria may become larger or smaller to show the change of results. For the same values of the first and second ranking criteria, the values of the third ranking criterion may become larger or smaller to present results changes. Hence, the first ranking criterion in Fig. 7 - 4 is non-increasing for all instances. The second and third ranking criteria may increase or decrease while the third ranking criterion has larger fluctuations than the second ranking criterion. To show the scheduling results more clearly for eight instances in set two, Fig. 7 - 5 illustrates the fuzzy Gantt chart of the best solution for instance Reman 2.

Table 7 - 3 Seven compared algorithms' results for five cases in set one

\begin{tabular}{|c|c|c|c|c|c|c|c|c|c|c|}
\hline \multirow{2}{*}{$\begin{array}{l}\text { Instance } \\
\text { Case1 }\end{array}$} & \multirow{2}{*}{$\frac{\text { Algorithm }}{\text { FDHS }}$} & \multicolumn{3}{|c|}{ Average value } & \multicolumn{3}{|c|}{ Best value } & \multicolumn{3}{|c|}{ Worst value } \\
\hline & & 19.9 & 29.6 & 40.6 & 17 & 29 & 39 & 19 & 31 & 44 \\
\hline & EDA & 20.3 & 30.5 & 41.6 & 20 & 28 & 40 & 22 & 32 & 43 \\
\hline & HABC & 21 & 32 & 43.6 & 19 & 30 & 43 & 23 & 33 & 46 \\
\hline & CGA & 23.1 & 33.1 & 43.4 & 21 & 29 & 41 & 25 & 37 & 47 \\
\hline & DIGA & 22.5 & 32.7 & 43.3 & 21 & 31 & 40 & 25 & 36 & 48 \\
\hline & PEGA & 25 & 35.1 & 47.2 & 23 & 31 & 42 & 29 & 40 & 50 \\
\hline & $\mathrm{PSO}+\mathrm{SA}$ & 26.2 & 36.9 & 47.7 & 25 & 32 & 40 & 27 & 41 & 54 \\
\hline \multirow[t]{7}{*}{ Case2 } & FDHS & 32.1 & 46.2 & 57.3 & 30 & 46 & 58 & 35 & 46 & 57 \\
\hline & EDA & 33.7 & 46.9 & 57.9 & 32 & 46 & 57 & 34 & 48 & 58 \\
\hline & HABC & 33 & 47.8 & 62.2 & 33 & 46 & 58 & 36 & 48 & 65 \\
\hline & CGA & 35 & 47.1 & 60.6 & 32 & 47 & 57 & 38 & 49 & 64 \\
\hline & DIGA & 33.4 & 47.5 & 62.1 & 31 & 47 & 59 & 38 & 50 & 66 \\
\hline & PEGA & 36.9 & 51 & 65.9 & 34 & 45 & 60 & 38 & 55 & 72 \\
\hline & $\mathrm{PSO}+\mathrm{SA}$ & 36.7 & 51.2 & 65.2 & 34 & 45 & 60 & 39 & 54 & 74 \\
\hline Case3 & FDHS & 31.6 & 45.9 & 59.9 & 31 & 45 & 58 & 33 & 48 & 62 \\
\hline
\end{tabular}




\begin{tabular}{llccccccccc}
\hline \multirow{5}{*}{ EDA } & 32.8 & 47.2 & 62.9 & 31 & 46 & 60 & 34 & 49 & 66 \\
& HABC & 33.9 & 50.8 & 67.3 & 33 & 47 & 64 & 36 & 54 & 70 \\
& CGA & 36.4 & 50.8 & 66 & 34 & 47 & 63 & 38 & 53 & 71 \\
& DIGA & 36.1 & 51.5 & 67.5 & 36 & 47 & 64 & 40 & 55 & 73 \\
& PEGA & 40.6 & 56.4 & 73.3 & 38 & 51 & 66 & 40 & 59 & 77 \\
& PSO+SA & 38.6 & 54.4 & 70 & 36 & 51 & 65 & 40 & 57 & 75 \\
& & & & & & & & & & \\
Case4 & FDHS & $\mathbf{2 4 . 1}$ & $\mathbf{3 6 . 1}$ & $\mathbf{5 0 . 9}$ & $\mathbf{2 4}$ & $\mathbf{3 5}$ & $\mathbf{4 8}$ & $\mathbf{2 6}$ & $\mathbf{3 7}$ & $\mathbf{5 3}$ \\
& EDA & 24.8 & 37.2 & 51.9 & 21 & 36 & 50 & 24 & 39 & 57 \\
& HABC & 25.5 & 40 & 56.3 & 23 & 38 & 53 & 25 & 44 & 59 \\
& CGA & 27.4 & 40.4 & 55 & 26 & 37 & 51 & 29 & 42 & 59 \\
& DIGA & 29.6 & 42.4 & 56.9 & 28 & 40 & 56 & 30 & 46 & 63 \\
& PEGA & 34.3 & 48.8 & 65.7 & 34 & 46 & 63 & 35 & 50 & 68 \\
& PSO+SA & 33.6 & 47.9 & 64.5 & 32 & 45 & 62 & 34 & 49 & 68 \\
& & & & & & & & & & \\
& FDHS & $\mathbf{3 7 . 9}$ & $\mathbf{5 5 . 8}$ & $\mathbf{7 7 . 8}$ & $\mathbf{3 6}$ & $\mathbf{5 4}$ & $\mathbf{7 4}$ & 42 & 59 & 84 \\
& EDA & 38.6 & 56.9 & 78.3 & 36 & 55 & 73 & $\mathbf{4 0}$ & $\mathbf{6 0}$ & $\mathbf{8 1}$ \\
& CGA & 47 & 65.4 & 86 & 42 & 62 & 82 & 49 & 70 & 91 \\
& DIGA & 45.8 & 66.3 & 88.7 & 42 & 63 & 84 & 49 & 71 & 92 \\
& PEGA & 50.3 & 74 & 96.5 & 48 & 68 & 94 & 50 & 74 & 100 \\
& PSO+SA & 51.2 & 74.6 & 97.6 & 48 & 72 & 93 & 52 & 73 & 101 \\
\hline
\end{tabular}

Table 7 - 4 Seven algorithms' CPU time (s) for five cases in set one

\begin{tabular}{llrrrrr}
\hline Algorithm & CPU & Case1 & Case2 & Case3 & Case4 & Case5 \\
\hline FDHS & $2.40 \mathrm{GHz}$ & 1.00 & 1.03 & 1.30 & 1.34 & 2.65 \\
EDA & $2.3 \mathrm{GHz}$ & 3.65 & 3.63 & 4.86 & 4.56 & 9.83 \\
$\mathrm{HABC}$ & $2.83 \mathrm{GHz}$ & 9.87 & 10.88 & 14.8 & 13.85 & - \\
CGA & $1.7 \mathrm{GHz}$ & 8.29 & 8.26 & 10.66 & 10.77 & 23.87 \\
DIGA & $1.7 \mathrm{GHz}$ & 15.36 & 15.57 & 18.87 & 19.02 & 37.82 \\
PEGA & $1.7 \mathrm{GHz}$ & 12.56 & 12.67 & 15.23 & 15.71 & 30.15 \\
PSO+SA & $1.7 \mathrm{GHz}$ & 12.4 & 12.33 & 15.24 & 15.66 & 30.9 \\
\hline
\end{tabular}

In addition, the average CPU times and generations for eight instances in set two are shown in Table 7 - 6. It can be seen from Table 7 - 6 that average CPU times for 1000 generations are less than 3 seconds for instances Reman 1 to Reman 4. For instances Reman 5 and Reman 6, the average CPU times are more than 20 seconds. The reason is that the instance size becomes larger and the maximum generation increases from 1000 to 4000 . The instance sizes of Reman 7 and Reman 8 are 20-jobs, 10machines, 308-operations and 20-jobs, 15-machines, 355-operations. The average CPU time is 73.83 seconds for Reman 7 and 103.56 seconds for Reman 8.

In a summary, the proposed FDHS algorithm has better performance than six compared algorithms for five cases in set one. Compared to MinEnd heuristic, FDHS can improve the fuzzy completion time results more than $30 \%$ for all instances in set two. At the same time, the average CPU times of 
FDHS algorithm are less than those of six compared algorithms for 5 cases in set one. For the eight instances in set two, FDHS also has very limited average CPU time.

Table 7 - 5 FDHS and MinEnd results for eight instances in set two

\begin{tabular}{|c|c|c|c|c|c|c|c|c|c|c|c|c|c|}
\hline \multirow{2}{*}{$\begin{array}{l}\text { Instance } \\
\operatorname{Re} 1\end{array}$} & \multirow{2}{*}{$\begin{array}{l}\text { Algorithm } \\
\text { FDHS }\end{array}$} & \multicolumn{3}{|c|}{ Average value } & \multicolumn{3}{|c|}{ Ave impro (\%) } & \multicolumn{3}{|c|}{ Best value } & \multicolumn{3}{|c|}{ Worst value } \\
\hline & & 17.4 & 26.0 & 34.7 & 30.6 & 33.1 & 32.9 & 19 & 26 & 33 & 18 & 26 & 36 \\
\hline & MinEnd & 25.1 & 38.9 & 51.8 & & & & 18 & 30 & 42 & 37 & 52 & 68 \\
\hline \multirow[t]{2}{*}{$\operatorname{Re} 2$} & FDHS & 24.7 & 40.7 & 56.8 & 34.0 & 33.4 & 33.1 & 17 & 39 & 58 & 28 & 43 & 59 \\
\hline & MinEnd & 37.5 & 61.2 & 84.9 & & & & 31 & 51 & 73 & 48 & 72 & 96 \\
\hline \multirow[t]{2}{*}{$\operatorname{Re} 3$} & FDHS & 36.8 & 59.1 & 80.3 & 43.1 & 40.7 & 39.7 & 37 & 58 & 77 & 43 & 60 & 79 \\
\hline & MinEnd & 64.8 & 99.7 & 133.2 & & & & 57 & 89 & 115 & 69 & 110 & 144 \\
\hline \multirow[t]{2}{*}{$\operatorname{Re} 4$} & FDHS & 34.3 & 51.0 & 70.2 & 35.4 & 37.0 & 37.1 & 31 & 49 & 70 & 35 & 54 & 75 \\
\hline & MinEnd & 53.2 & 81 & 111.7 & & & & 38 & 67 & 94 & 63 & 104 & 147 \\
\hline \multirow[t]{2}{*}{$\operatorname{Re} 5$} & FDHS & 52.6 & 84.4 & 116.1 & 40.3 & 39.9 & 40.0 & 53 & 82 & 112 & 55 & 86 & 121 \\
\hline & MinEnd & 88.1 & 140.6 & 193.6 & & & & 82 & 132 & 184 & 103 & 150 & 206 \\
\hline \multirow[t]{2}{*}{$\operatorname{Re} 6$} & FDHS & 44.5 & 72.6 & 98.5 & 37.1 & 38.8 & 39.2 & 44 & 69 & 94 & 52 & 76 & 98 \\
\hline & MinEnd & 70.8 & 118.7 & 162 & & & & 62 & 100 & 139 & 67 & 134 & 186 \\
\hline \multirow[t]{2}{*}{$\operatorname{Re} 7$} & FDHS & 60.4 & 107.7 & 153.3 & 37.6 & 35.5 & 34.3 & 60 & 105 & 149 & 58 & 112 & 159 \\
\hline & MinEnd & 96.9 & 167.2 & 233.6 & & & & 94 & 152 & 214 & 100 & 179 & 254 \\
\hline \multirow[t]{2}{*}{$\operatorname{Re} 8$} & FDHS & 57.7 & 92.3 & 126.6 & 32.0 & 34.5 & 34.3 & 56 & 89 & 122 & 57 & 95 & 133 \\
\hline & MinEnd & 84.9 & 141.1 & 192.8 & & & & 83 & 132 & 172 & 95 & 153 & 206 \\
\hline
\end{tabular}

Table 7 - 6 Average CPU time and generation of FDHS for eight instances in set two

\begin{tabular}{llllll}
\hline Instance & Cpu time (s) & Generation & Instance & Cpu time (s) & Generation \\
\hline Reman 1 & 0.40 & 1000 & Reman 5 & 22.38 & 4000 \\
Reman 2 & 1.44 & 1000 & Reman 6 & 25.97 & 4000 \\
Reman 3 & 1.89 & 1000 & Reman 7 & 73.83 & 4000 \\
Reman 4 & 2.68 & 1000 & Reman 8 & 103.56 & 4000 \\
\hline
\end{tabular}

Notes:2.40GHz CPU 


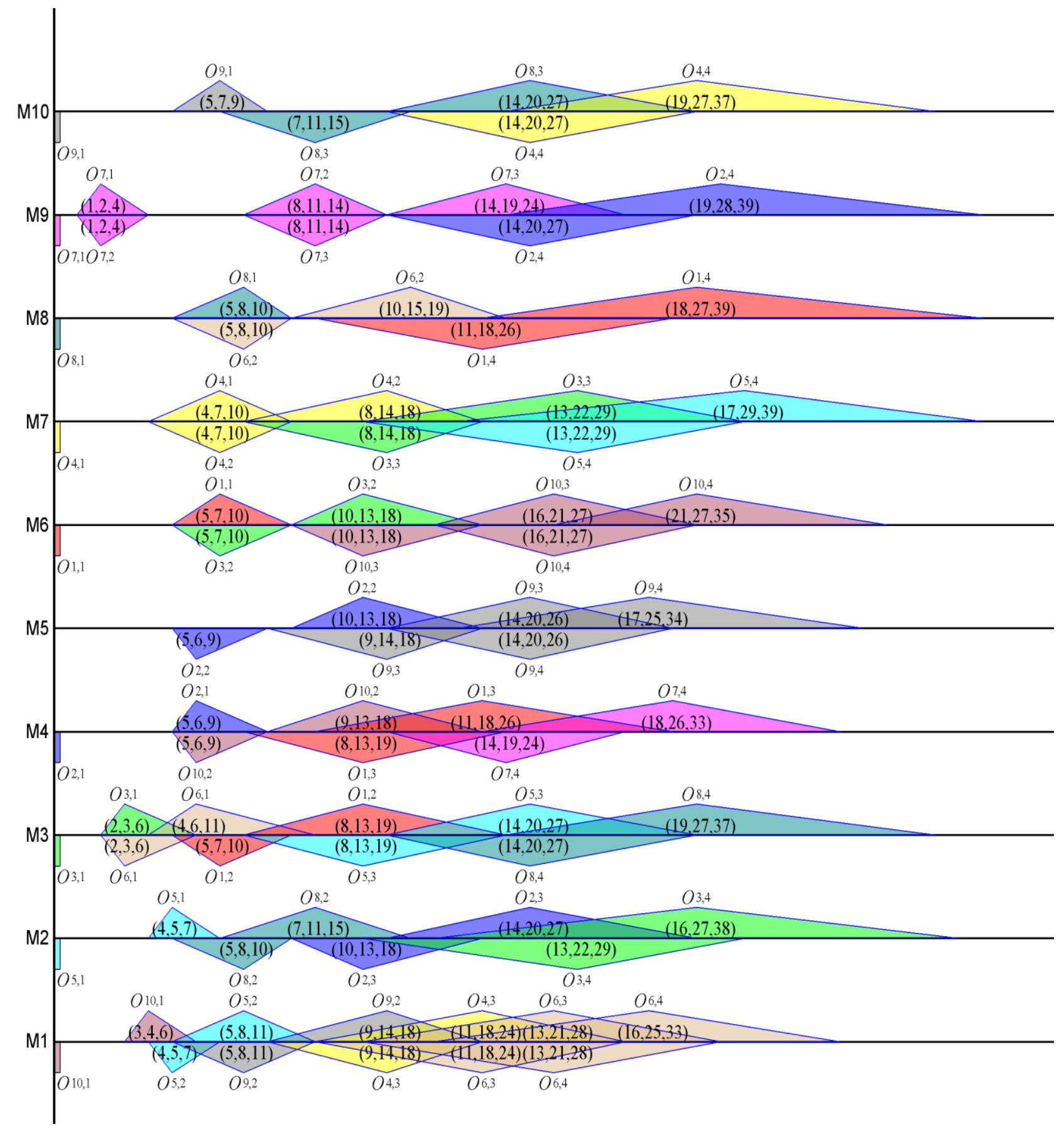

Fig. 7 - 3 Best solution's fuzzy Gantt chart of case 1 


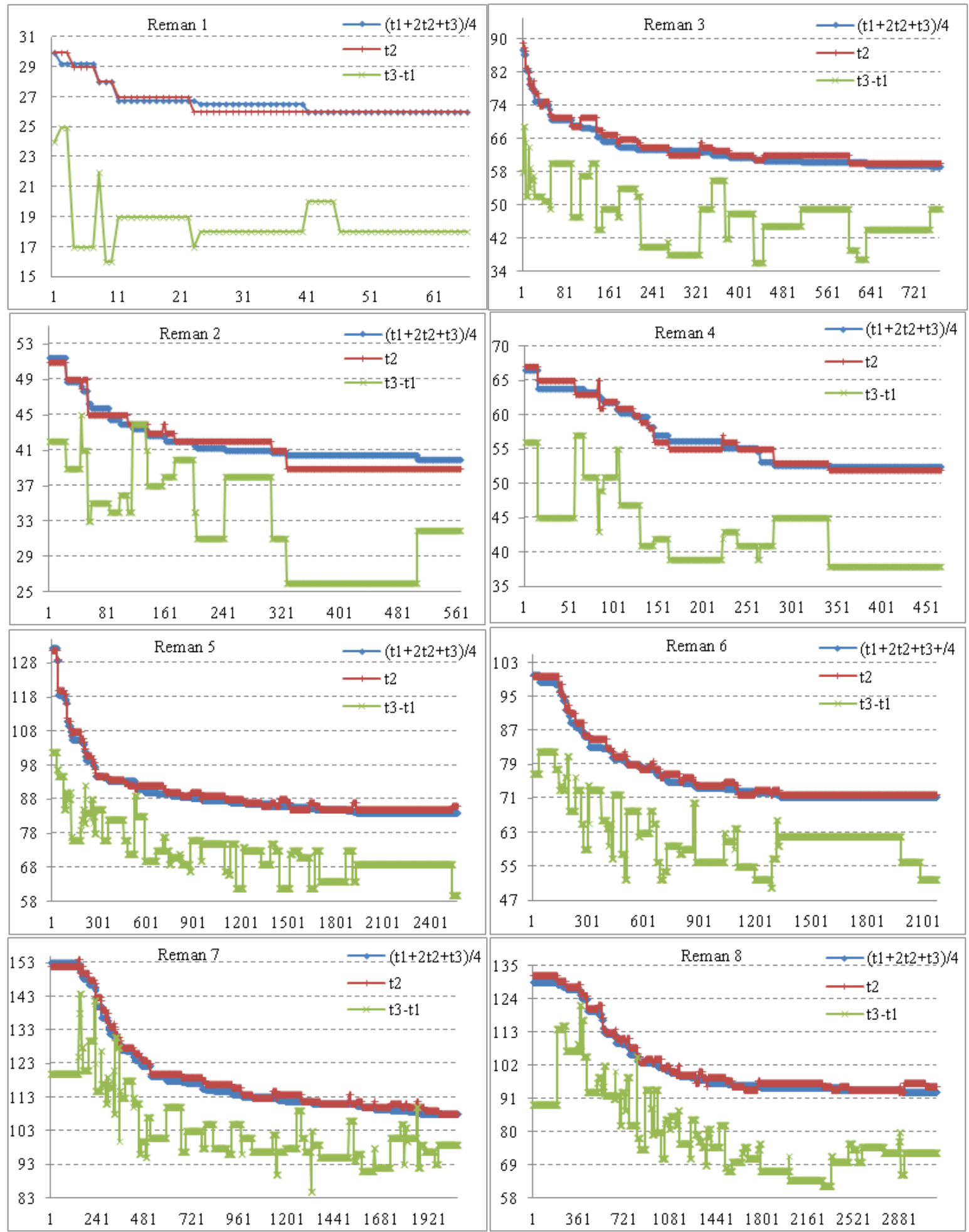

Fig. 7 - 4 Convergence curve of FDHS for eight instances in set two 


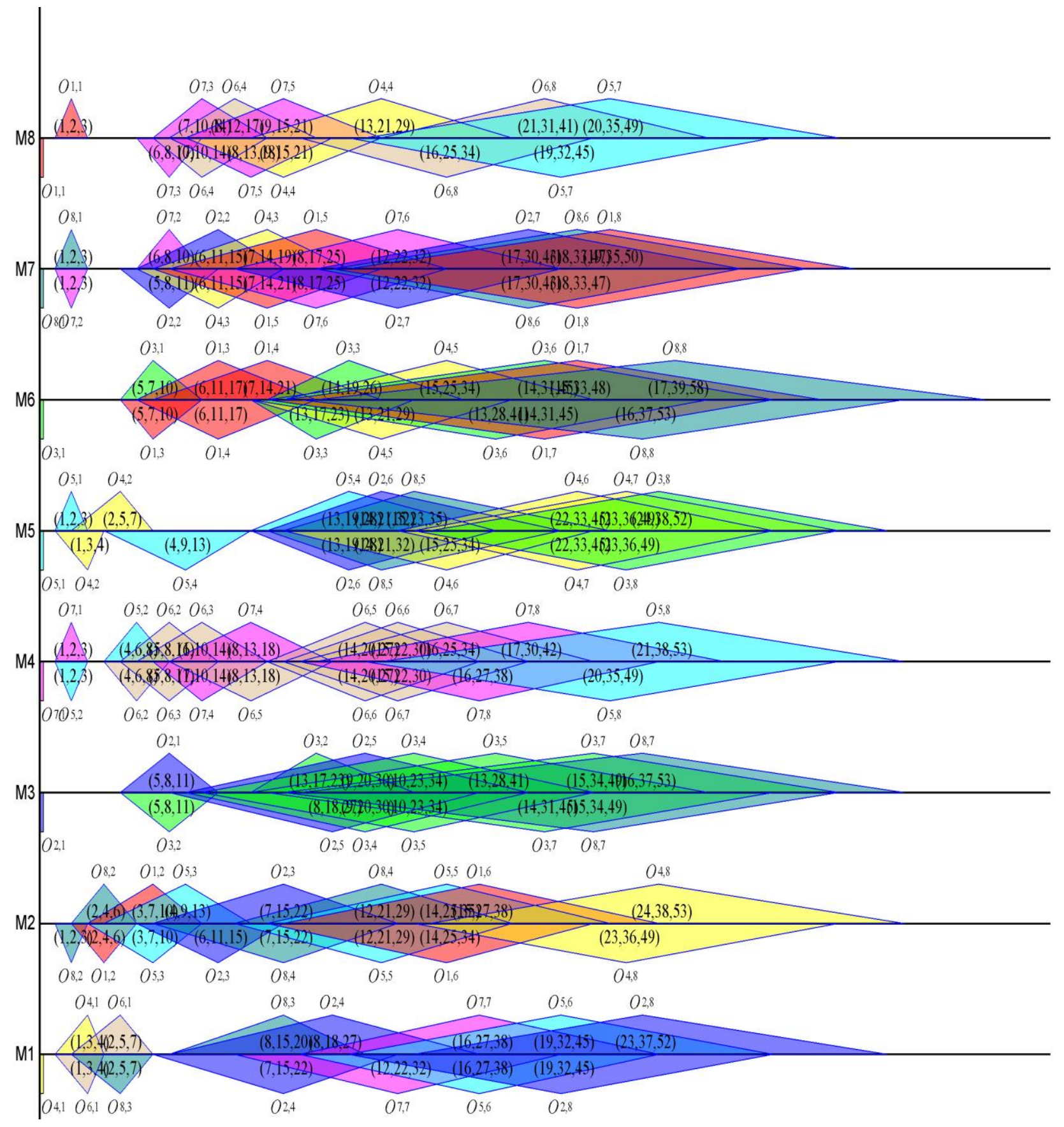

Fig. 7 - 5 Best solution's fuzzy Gantt chart of instance Reman 2 


\subsection{Conclusions}

In this Chapter, a fuzzy discrete harmony search (FDHS) algorithm was proposed for solving flexible job shop scheduling problem with fuzzy processing time. The objective was to minimize the maximum fuzzy completion time. The proposed MinEnd heuristic was evaluated by comparing against the random rule and four existing heuristics. The FDHS compared against six existing algorithms for solving five benchmark cases. The results and comparisons showed superior performance of FDHS. As an example, the fuzzy Gantt chart of one case's new best solution was given. In addition, eight instances from remanufacturing were solved by MinEnd heuristic and FDHS algorithm. FDHS improved results by MinEnd obviously. The computer CPU time of FDHS algorithm was very low. The experiments also showed the convergence performance of FDHS algorithm. Fuzzy Gantt chart of one remanufacturing instance was shown as an example. 


\section{Chapter 8}

\section{FABC Algorithm for Multi-objective FJSSP with Fuzzy Processing Time}

\subsection{Introduction}

This Chapter addresses multi-objective flexible job shop scheduling problem (FJSSP) with fuzzy processing time. The uncertainty in the processing time is one of the seven characteristics in remanufacturing. An effective fuzzy artificial bee colony (FABC) algorithm is proposed for FJSSP with fuzzy processing time. The objectives are to minimize the maximum fuzzy completion time and the maximum fuzzy machine workload. The three-value element encoding and decoding method is used to show the solution in FABC algorithm. The MinEnd heuristic proposed in Chapter 7 is employed to initialize population. Extensive computational experiments are carried out using five benchmark cases with eight instances from remanufacturing. The proposed heuristic rule is evaluated using five benchmark cases for minimizing the maximum fuzzy completion time and the maximum fuzzy machine workload objectives. FABC algorithm is compared to FDHS algorithm and six metaheuristics for maximum fuzzy completion time criterion. For maximum fuzzy machine workload objective, FABC algorithm is compared to FDHS algorithm and six heuristics. The results and comparisons demonstrate improved performance of FABC algorithm for solving multi-objective flexible job shop scheduling problem with fuzzy processing time. 
The rest of this Chapter is organized as follows: Section 8.2 briefly describes the two objectives of FJSSP with fuzzy processing time constraint. The proposed FABC algorithm is represented in Section 8.3. Section 8.4 is devoted to experimental results and comparisons. We conclude this Chapter in Section 8.5.

\subsection{FJSSP with fuzzy processing time}

The FJSSP with fuzzy processing time means that the operation processing time is not an exact value and the processing time is shown as a triangular fuzzy number (TFN) as follows:

$$
t_{i, j, k}=\left(t_{i, j, k}^{1}, t_{i, j, k}^{2}, t_{i, j, k}^{3}\right)
$$

where $t_{i, j, k}^{1}, t_{i, j, k}^{2}$ and $t_{i, j, k}^{3}$ are three probable processing time of operation $\boldsymbol{O}_{i, j}$ on machine $M_{k}$. The fuzzy completion time of operation $\boldsymbol{O}_{i, j}$ is a TFN as follows:

$$
C_{i, j}=\left(C_{i, j}^{1}, C_{i, j}^{2}, C_{i, j}^{3}\right)
$$

where $C_{i, j}^{1}, C_{i, j}^{2}$ and $C_{i, j}^{3}$ are three probable completion time of operation $\boldsymbol{O}_{i, j}$.

The fuzzy machine workload of machine $M_{k}$ is also a TFN as follows:

$$
w_{k}=\left(w_{k}^{1}, w_{k}^{2}, w_{k}^{3}\right)
$$

$w_{k}^{1}, w_{k}^{2}$ and $w_{k}^{3}$ are three probable machine workload of machine $M_{k}$.

The objective is to minimize maximum fuzzy completion time and maximum fuzzy machine workload. The maximum fuzzy completion time denoted by $C_{M}$ can be calculated by the following formula:

$$
\text { Min } C_{M}=\max _{1 \leq i \leq n}\left\{C_{i}\right\}
$$

where $C_{i}$ is the fuzzy completion time of job $J_{i}$.

The maximum fuzzy machine workload denoted by $W_{M}$, can be calculated by:

$$
\operatorname{Min} W_{M}=\max _{1 \leq k \leq m}\left\{W_{k}\right\}
$$

where $W_{k}$ is the workload of machine $M_{k}$.

\subsection{FABC for FJSSP with fuzzy processing time}

\subsubsection{Initializing}

The quality of initial population often affects the convergence of meta-heuristic for FJSSP. It is important to generate a good quality initial population. Many heuristic rules are proposed for machine assignment and operation sequencing in FJSSP, such as, local minimizing processing time (LS) rule, global minimizing processing time rule (GS), most work remaining rule (MReW) and most number of operations remaining (MReO) rule [16]. In this Chapter, we develop MinEnd heuristic to initialize 
population. In this rule, the operation sequence is generated randomly. The processing machine is assigned based on the operation order in operation sequence. For each operation, the machine with the minimum fuzzy completion time will be selected to process this operation.

\subsubsection{Employed bee phase}

In this phase, employed bee $X_{i}$ generates a new food source $X_{n e w}$. If the fitness of $X_{\text {new }}$ is equal to or better than that of $X_{i}, X_{n e w}$ will replace $X_{i}$ as a new food source; otherwise, $X_{i}$ is retained. To adapt FJSSP with fuzzy processing time and encoding rule, the procedure for new food source is shown as follows.

Procedure: Generating new food source

If $(\mathrm{r} 1<\mathrm{P} 1) \quad / / \mathrm{r} 1$ is a random in $[0,1], \mathrm{P} 1$ is the probability to generate new food source

Select two employed bee $X_{p}$ and $X_{q}, X_{p} \neq X_{q} \neq X_{i}$.

If $(\mathrm{r} 2<\mathrm{P} 2) / / \mathrm{r} 2$ is a random in $[0,1], \mathrm{P} 2$ is the probability to select a better food source from $X_{p}$ and $X_{q}$

If the fitness of $X_{p}$ is better than that of $X_{q}, X_{j}=X_{p}$; else $X_{j}=X_{q}$.

Else

If the fitness of $X_{p}$ is worse than that of $X_{q}, X_{j}=X_{p}$; else $X_{j}=X_{q}$.

For $\mathrm{k}=1$ to toper $/ /$ toper is the total operation number

If $(\mathrm{r} 3<\mathrm{P} 3) / / \mathrm{r} 3$ is a random in $[0,1], \mathrm{P} 3$ is the probability to select element from $X_{j}$

$$
X_{n e w}^{k}=X_{j}^{k}
$$

Else

$$
X_{\text {new }}^{k}=X_{i}^{k}
$$

Repair $X_{n e w}$ to make sure the operations of the same job can satisfy the processing precedence.

Else

For $\mathrm{k}=1$ to toper $/ /$ toper is the total operation number

If $(\mathrm{r} 4<\mathrm{P} 4) \quad / / \mathrm{r} 4$ is a random in $[0,1], \mathrm{P} 4$ is the probability for the minimum processing time machine

If current machine of $X_{i}^{k}$ has minimum processing time,

Select another machine randomly for $X_{i}^{k}$

Else 
Select minimum processing time machine for $X_{i}^{k}$

Else

Select two operations $X_{i}^{a}$ and $X_{i}^{b}, a<b$.

If $X_{i}^{a}$ and $X_{i}^{b}$ are different jobs' operations

Insert $X_{i}^{a}$ at $X_{i}^{b}$ position.

Repair $X_{\text {new }}$ to make sure the operations of the same job can satisfy the processing precedence.

End

One employed bee can generate a new food source by selecting operations from different bees or by changing assigned machines. The complexity by operation sequence is $O\left(\log _{2} \sum_{i=1}^{n} q_{i} *\right.$ $\left.\log _{2} \sum_{i=1}^{n} q_{i}\right)$ while the complexity by changing machine is $O\left(\left(\sum_{i=1}^{n} q_{i}\right)\right.$. The computational to repair the new food source is $O\left(\left(\sum_{i=1}^{n} q_{i} * \log _{2} \sum_{i=1}^{n} q_{i}\right)\right.$.

\subsubsection{Onlooker bee phase}

In onlooker bee phase, the fitness of all the employed bees is evaluated. A food source $X_{i}$ is selected depending on its probability value $p_{i}$ calculated by expression (3-8) shown in Chapter 3 . The procedure to select a food source is shown as follows.

Procedure: Select a food source

Select two employed bee $X_{p}$ and $X_{q}, X_{p} \neq X_{q}$.

If $(\mathrm{r} 2<\mathrm{P} 2)$

If the fitness of $X_{p}$ is better than that of $X_{q}, X_{i}=X_{p}$; else, $X_{i}=X_{q}$.

Else

If the fitness of $X_{p}$ is worse than that of $X_{q}, X_{i}=X_{p}$; else, $X_{i}=X_{q}$.

End

\subsubsection{Scout bee phase}

If a food source $X_{i}$ cannot be improved through a predetermined number of trials Limit, the food source is to be abandoned and the corresponding employed bee becomes a scout. A new food source will be generated randomly.

\subsubsection{Procedure of FABC algorithm}


In the FABC algorithm, the proposed MinEnd heuristic, GS heuristic rule, and random rule are used to initial population. In this Chapter, $60 \%$ solutions in population are generated randomly, $20 \%$ solutions by MinEnd heuristic and $20 \%$ solutions by GS heuristic rule. In the procedure to generate new food source, changing processing machine operator and operation inserting operator are employed for exploitation search. Better or worse food source selection and scout bee operator are used for exploration search. Based on the special design above, the procedure of FABC algorithm is as follows.

Procedure: FABC algorithm

Step 1: Set parameters, employed bee number, onlooker bee number, scout bee number, probability P1, P2, P3 and P4.

Step2: Initialize population using MinEnd heuristic, global minimizing processing time rule and random rule.

Step3: Perform employed bee phase.

Step4: Perform onlooker bee phase.

Step5: Update the best solution.

Step6: If Limit is met, perform scout bee phase

Step7: If the stop criterion is not satisfied, go to Step 3; else, output the best solution.

\subsection{Experiment evaluation and comparisons}

\subsubsection{Experiment setup}

To evaluate the performance of proposed FABC algorithm for minimizing the maximum fuzzy completion time and the maximum fuzzy machine workload criteria, experimental evaluation and comparisons are conducted. Two sets of instances are evaluated in this Chapter. Set one consist five FJSSP with fuzzy processing time cases $[82,147]$. The sizes are ranging from 10-jobs, 10-machines and 40-operations to 15-jobs, 10-machines and 80-operations. Set two includes eight instances from remanufacturing industry. The size is from 5-jobs, 4-machines and 23-operations to 20-jobs, 15machines and 355-operations. The proposed FABC algorithm is coded in C++ and run on Intel 2.40 GHz PC with 2 GB memory. The parameters are set based on our previous research and the compared algorithms as follows: employed bee 50; onlooker bee 100, scout bee 20, Limit 50, P1=0.8, P2=0.95, $\mathrm{P} 3=0.2$ and $\mathrm{P} 4=0.5$. For instances in set one and the previous four instances in set two, the generation is set to 1000. For the last four instances in set two, the generation is set to 4000 because the problem size of these four instances is larger than that of other instances. All experiments are carried out with 30 replications.

\subsubsection{Testing MinEnd heuristic}

To test MinEnd heuristic performance, five cases in set one are evaluated by MinEnd heuristic, GS, LS, MReW, MReO and random six heuristic rules. The maximum fuzzy completion time results are 
shown in Table 8 - 1 while the maximum fuzzy machine workload results are shown in Table 8 - 2 . For the two objectives, the best values, the average of the expected values and worst values in 30 runs are counted.

Compared to random rule, $\mathrm{MReW}$ and $\mathrm{MReO}$ rules cannot improve maximum fuzzy completion time and maximum fuzzy machine workload results. MinEnd, GS and LS heuristic rules can get higher quality results than $\mathrm{MReW}, \mathrm{MReO}$ and random rules. Among the six heuristics, MinEnd can find the best maximum fuzzy completion time results for all five cases except the best value of case1. MinEnd heuristic gets the best value $(42,53,69)$ for case 1 while LS heuristic rule obtains the best value $(22$, 46, 58). GS rule can find the maximum fuzzy completion time results second only to MinEnd heuristic. For maximum fuzzy machine workload objective, GS heuristic rule obtains the best results for all five cases except the best value of case 3 . GS finds the best value $(32,44,55)$ for case 3 while MinEnd heuristic finds the best value $(27,42,56)$. MinEnd heuristic can get the maximum fuzzy machine workload results second only to the GS rule. To show the best results clearly, the best results data in Table 8 - 1 and Table 8 - 2 are in bold. Considering the two objectives, MinEnd and GS are two effective heuristic rules for FJSSP with fuzzy processing time.

Table 8 - 1 Maximum fuzzy completion time of five cases by six heuristics

\begin{tabular}{llrrrrrrrrr}
\hline Instance & Algorithm & \multicolumn{3}{c}{ Average value } & \multicolumn{3}{c}{ Best value } & \multicolumn{3}{c}{ Worst value } \\
\hline Case1 & MinEnd & $\mathbf{4 5 . 6}$ & $\mathbf{6 1 . 9}$ & $\mathbf{8 1 . 0}$ & 42 & 53 & 69 & $\mathbf{5 1}$ & $\mathbf{7 3}$ & $\mathbf{9 4}$ \\
& GS & 52.0 & 74.3 & 96.2 & 38 & 53 & 67 & 76 & 113 & 150 \\
& LS & 49.4 & 71.2 & 90.9 & $\mathbf{2 2}$ & $\mathbf{4 6}$ & $\mathbf{5 8}$ & 63 & 92 & 125 \\
& MReW & 59.3 & 88.9 & 117.5 & 49 & 75 & 102 & 75 & 109 & 142 \\
& MReO & 59.7 & 88.8 & 117.0 & 54 & 79 & 103 & 75 & 110 & 143 \\
& Random & 59.2 & 88.0 & 116.2 & 49 & 75 & 102 & 69 & 102 & 138 \\
& & & & & & & & & & \\
Case2 & MinEnd & $\mathbf{4 5 . 6}$ & $\mathbf{6 1 . 9}$ & $\mathbf{8 1 . 0}$ & $\mathbf{4 2}$ & $\mathbf{5 3}$ & $\mathbf{6 9}$ & $\mathbf{5 1}$ & $\mathbf{7 3}$ & $\mathbf{9 4}$ \\
& GS & 80.7 & 108.3 & 138.5 & 50 & 69 & 90 & 108 & 147 & 189 \\
& LS & 81.5 & 109.9 & 140.3 & 57 & 77 & 97 & 115 & 154 & 196 \\
& MReW & 98.4 & 132.6 & 168.8 & 89 & 118 & 151 & 114 & 152 & 195 \\
& MReO & 102.9 & 139.3 & 177.9 & 89 & 118 & 151 & 127 & 169 & 216 \\
& Random & 99.0 & 134.4 & 171.6 & 89 & 118 & 151 & 131 & 178 & 229 \\
& & & & & & & & & & \\
Case3 & MinEnd & $\mathbf{4 6 . 1}$ & $\mathbf{6 5 . 9}$ & $\mathbf{8 5 . 6}$ & $\mathbf{4 0}$ & $\mathbf{5 7}$ & $\mathbf{7 2}$ & $\mathbf{5 4}$ & $\mathbf{7 9}$ & $\mathbf{1 0 1}$ \\
& GS & 72.1 & 97.7 & 125.1 & 47 & 65 & 87 & 90 & 123 & 156 \\
& LS & 77.3 & 105.6 & 135.7 & 58 & 76 & 97 & 98 & 132 & 171 \\
& MReW & 88.3 & 117.9 & 151.5 & 68 & 91 & 117 & 109 & 142 & 185 \\
& MReO & 83.3 & 111.7 & 143.7 & 72 & 96 & 122 & 128 & 167 & 217 \\
& Random & 85.5 & 114.1 & 146.9 & 71 & 97 & 126 & 116 & 151 & 193 \\
& & & & & & & & & & \\
Case4 & MinEnd & $\mathbf{3 4 . 9}$ & $\mathbf{5 1 . 9}$ & $\mathbf{7 2 . 2}$ & $\mathbf{2 8}$ & $\mathbf{4 5}$ & $\mathbf{6 2}$ & $\mathbf{3 9}$ & $\mathbf{5 9}$ & $\mathbf{8 8}$ \\
& GS & 55.9 & 79.4 & 105.6 & 36 & 60 & 81 & 81 & 118 & 158 \\
& LS & 54.7 & 78.1 & 103.1 & 39 & 57 & 74 & 78 & 108 & 141
\end{tabular}




\begin{tabular}{rlrrrrrrrrr} 
& MReW & 64.4 & 87.7 & 113.4 & 54 & 70 & 90 & 86 & 126 & 163 \\
& MReO & 61.8 & 85.6 & 110.6 & 52 & 70 & 90 & 70 & 100 & 129 \\
& Random & 67.7 & 93.7 & 121.3 & 48 & 70 & 90 & 78 & 110 & 141 \\
Case5 & & & & & & & & & \\
& MinEnd & $\mathbf{5 3 . 7}$ & $\mathbf{7 8 . 5}$ & $\mathbf{1 0 8 . 2}$ & $\mathbf{4 5}$ & $\mathbf{6 8}$ & $\mathbf{9 4}$ & $\mathbf{6 1}$ & $\mathbf{9 3}$ & $\mathbf{1 2 6}$ \\
& GS & 80.9 & 113.5 & 150.4 & 53 & 83 & 113 & 103 & 142 & 194 \\
& LS & 81.2 & 111.3 & 146.8 & 90 & 73 & 100 & 114 & 158 & 204 \\
& MReW & 92.1 & 129.1 & 170.8 & 76 & 111 & 147 & 104 & 144 & 188 \\
& MReO & 95.5 & 134.1 & 177.5 & 85 & 114 & 157 & 113 & 155 & 207 \\
& Random & 94.4 & 132.7 & 174.7 & 83 & 114 & 149 & 134 & 190 & 247 \\
\hline
\end{tabular}

Table 8 - 2 Maximum fuzzy machine workload of five cases by six heuristics

\begin{tabular}{|c|c|c|c|c|c|c|c|c|c|c|}
\hline \multirow{2}{*}{$\begin{array}{l}\text { Ins.e } \\
\text { Case1 }\end{array}$} & \multirow{2}{*}{$\frac{\text { Algorithm }}{\text { MinEnd }}$} & \multicolumn{3}{|c|}{ Average value } & \multicolumn{3}{|c|}{ Best value } & \multicolumn{3}{|c|}{ Worst value } \\
\hline & & 23.0 & 34.2 & 45.9 & 21 & 30 & 40 & 26 & 41 & 54 \\
\hline & GS & 20.9 & 30.6 & 40.9 & 19 & 27 & 40 & 26 & 33 & 42 \\
\hline & LS & 33.0 & 50.0 & 64.0 & 33 & 50 & 64 & 33 & 50 & 64 \\
\hline & MReW & 45.0 & 66.0 & 85.4 & 30 & 44 & 57 & 66 & 92 & 114 \\
\hline & $\mathrm{MReO}$ & 42.3 & 62.6 & 81.3 & 27 & 41 & 55 & 65 & 102 & 132 \\
\hline & Random & 41.0 & 60.1 & 78.3 & 27 & 39 & 51 & 60 & 81 & 106 \\
\hline \multirow[t]{6}{*}{ Case2 } & MinEnd & 36.6 & 50.2 & 65.1 & 31 & 42 & 53 & 46 & 61 & 80 \\
\hline & GS & 32.1 & 44.5 & 57.4 & 28 & 40 & 50 & 33 & 47 & 65 \\
\hline & LS & 54.0 & 75.0 & 96.0 & 54 & 75 & 96 & 54 & 75 & 96 \\
\hline & MReW & 61.3 & 83.8 & 108.6 & 41 & 59 & 75 & 94 & 130 & 167 \\
\hline & $\mathrm{MReO}$ & 69.2 & 94.6 & 122.0 & 44 & 56 & 77 & 109 & 147 & 188 \\
\hline & Random & 65.0 & 88.9 & 113.9 & 42 & 60 & 78 & 93 & 129 & 165 \\
\hline \multirow[t]{6}{*}{ Case3 } & MinEnd & 36.4 & 51.4 & 66.6 & 27 & 42 & 56 & 40 & 61 & 78 \\
\hline & GS & 32.1 & 45.0 & 59.4 & 32 & 44 & 55 & 34 & 49 & 66 \\
\hline & LS & 49.0 & 72.0 & 89.0 & 49 & 72 & 89 & 49 & 72 & 89 \\
\hline & MReW & 63.5 & 88.9 & 115.2 & 48 & 66 & 85 & 93 & 130 & 164 \\
\hline & $\mathrm{MReO}$ & 67.1 & 94.5 & 123.2 & 39 & 58 & 77 & 95 & 131 & 172 \\
\hline & Random & 61.9 & 86.7 & 112.3 & 41 & 62 & 83 & 90 & 122 & 161 \\
\hline \multirow[t]{6}{*}{ Case4 } & MinEnd & 27.7 & 39.7 & 55.1 & 25 & 35 & 45 & 35 & 46 & 61 \\
\hline & GS & 23.9 & 34.9 & 48.4 & 23 & 33 & 45 & 28 & 38 & 52 \\
\hline & LS & 25.0 & 39.0 & 57.0 & 25 & 39 & 57 & 25 & 39 & 57 \\
\hline & MReW & 46.9 & 66.6 & 91.6 & 34 & 48 & 69 & 69 & 97 & 138 \\
\hline & $\mathrm{MReO}$ & 45.0 & 65.3 & 90.6 & 28 & 42 & 60 & 71 & 98 & 133 \\
\hline & Random & 41.8 & 60.2 & 82.5 & 30 & 42 & 61 & 60 & 83 & 110 \\
\hline \multirow[t]{6}{*}{ Case5 } & MinEnd & 45.2 & 64.9 & 88.7 & 42 & 60 & 80 & 50 & 73 & 97 \\
\hline & GS & 41.1 & 59.4 & 80.0 & 38 & 57 & 80 & 45 & 64 & 80 \\
\hline & LS & 52.0 & 76.0 & 109.0 & 52 & 76 & 109 & 52 & 76 & 109 \\
\hline & MReW & 80.9 & 114.5 & 154.0 & 65 & 88 & 118 & 110 & 155 & 206 \\
\hline & $\mathrm{MReO}$ & 80.9 & 113.2 & 151.4 & 55 & 86 & 119 & 105 & 141 & 189 \\
\hline & Random & 83.4 & 118.3 & 158.5 & 60 & 85 & 114 & 145 & 201 & 268 \\
\hline
\end{tabular}

8.4.3 Maximum fuzzy completion time objective 
For the five cases in set one, FABC algorithm is compared to six existing algorithms, EDA [77], HABC [137], CGA [82], DIGA [147], PEGA [16] and PSO+SA [23]. The results of average, best and worst values in 30 runs are shown in Table 8 - 3. It can be seen from Table 8 - 3 that the proposed FABC algorithm performs better than six compared algorithms. For five cases, FABC algorithm can obtain better average values and best values than the six compared algorithms. In 30 runs, FABC can find the minimum worst values for case 1 , case 2 case 3 and case 4 . EDA algorithm finds minimum worst value for case 5 . The best solution obtained by FABC for case 1 is $(19,28,39)$ and the fuzzy Gantt chart is illustrated in Fig. 8 - 1. The average CPU times of all the compared algorithms are listed in Table 8 - 4. Compared to the six existing algorithms, FABC has the minimum average CPU times. For case 1 to case 4, the average time of FABC is less than 1.5 seconds. For the largest case, case 5, the average CPU time is just 2.41 seconds. Although the CPU frequencies are different among seven algorithms, we can conclude that the proposed FABC algorithm is the most efficient one.

The FABC algorithm is also compared to the FDHS algorithm proposed in Chapter 7. It can be seen from Table 8 - 3 that the FABC algorithm improves some measures' results of FDHS algorithm for five cases. FDHS algorithm gets better average and best results for case 2 than those by FABC algorithm. FABC algorithm obtains competitive results for all other cases and comparison measures except the worst measure of case 4. In addition, the FABC and FDHS algorithms have the same average CPU time for case 1. FABC has smaller average CPU times than FDHS for case 2 and case 5. For case 3 and case 4, FABC has larger average CPU times than FDHS algorithm. These differences between FDHS and FABC are not very obvious.

Literature [149] and [150] proposed SNSA and HGTS algorithms for FJSSP with fuzzy processing time. Except the five cases above, SNSA and HGTS solved case 6 from literature [149]. In literature [150], HGTS solved the expected makespan and the results are compared to those by SNSA. Among all compared algorithms in [150], only SNSA and HGTS solved case 6 for excepted makespan. Hence, FDHS and FABC are compared to SNSA and HGTS for case 6 with expected makespan objective. SNSA, HGTS, FDHS and FABC run 30 repeats for case 6. The minimum results and average results of these four algorithms are shown in Table 8 -3. To compare with SNSA and HGTS, the format of the results for case 6 is based on the ranking operation of triangle fuzzy number in Section 3.3.2 of Chapter 3. The average computation times are shown in Table 8 -4. Compared to SNSA, FDHS and FABC obtained better minimum results and average results for the sixth case. SNSA is run on a $2 \mathrm{~GB}$ RAM 2.2 GHz computer while FDHS and FABC are run on a $2.4 \mathrm{GHz}$ computer. FDHS and FABC have smaller execution time than SNSA for case 6. Compared to HGTS, FDHS and FABC do not obtained better than HGTS. However, the differences among the three results are not obvious. HGTS needs much more computer CPU time than FDHS and FABC even the running times are not directly 
comparable due to difference in target machines. The running time of FDHS and FABC are 2.46 to 2.48 seconds while the running time of HGTS is larger than 50 seconds. It means that FDHS and FABC can obtain solutions that are very near to optimal solutions in very short CPU time.

Table 8 - 3 Maximum fuzzy completion time by eight algorithms for five cases

\begin{tabular}{|c|c|c|c|c|c|c|c|c|c|c|}
\hline \multirow{2}{*}{$\begin{array}{l}\text { Instance } \\
\text { Case1 }\end{array}$} & \multirow{2}{*}{$\begin{array}{l}\text { Algorithm } \\
\text { FABC }\end{array}$} & \multicolumn{3}{|c|}{ Average value } & \multicolumn{3}{|c|}{ Best value } & \multicolumn{3}{|c|}{ Worst value } \\
\hline & & 20.1 & 29.4 & 40.3 & 19 & 28 & 39 & 22 & 30 & 42 \\
\hline & FDHS & 19.9 & 29.6 & 40.6 & 17 & 29 & 39 & 19 & 31 & 44 \\
\hline & EDA & 20.3 & 30.5 & 41.6 & 20 & 28 & 40 & 22 & 32 & 43 \\
\hline & HABC & 21 & 32 & 43.6 & 19 & 30 & 43 & 23 & 33 & 46 \\
\hline & CGA & 23.1 & 33.1 & 43.4 & 21 & 29 & 41 & 25 & 37 & 47 \\
\hline & DIGA & 22.5 & 32.7 & 43.3 & 21 & 31 & 40 & 25 & 36 & 48 \\
\hline & PEGA & 25 & 35.1 & 47.2 & 23 & 31 & 42 & 29 & 40 & 50 \\
\hline & $\mathrm{PSO}+\mathrm{SA}$ & 26.2 & 36.9 & 47.7 & 25 & 32 & 40 & 27 & 41 & 54 \\
\hline \multirow[t]{8}{*}{ Case2 } & FABC & 32.3 & 46.2 & 57.3 & 33 & 45 & 58 & 35 & 46 & 57 \\
\hline & FDHS & 32.1 & 46.2 & 57.3 & 30 & 46 & 58 & 35 & 46 & 57 \\
\hline & EDA & 33.7 & 46.9 & 57.9 & 32 & 46 & 57 & 34 & 48 & 58 \\
\hline & HABC & 33 & 47.8 & 62.2 & 33 & 46 & 58 & 36 & 48 & 65 \\
\hline & CGA & 35 & 47.1 & 60.6 & 32 & 47 & 57 & 38 & 49 & 64 \\
\hline & DIGA & 33.4 & 47.5 & 62.1 & 31 & 47 & 59 & 38 & 50 & 66 \\
\hline & PEGA & 36.9 & 51 & 65.9 & 34 & 45 & 60 & 38 & 55 & 72 \\
\hline & $\mathrm{PSO}+\mathrm{SA}$ & 36.7 & 51.2 & 65.2 & 34 & 45 & 60 & 39 & 54 & 74 \\
\hline \multirow[t]{8}{*}{ Case 3} & FABC & 31.8 & 45.8 & 59.6 & 31 & 45 & 57 & 33 & 47 & 63 \\
\hline & FDHS & 31.6 & 45.9 & 59.9 & 31 & 45 & 58 & 33 & 48 & 62 \\
\hline & EDA & 32.8 & 47.2 & 62.9 & 31 & 46 & 60 & 34 & 49 & 66 \\
\hline & HABC & 33.9 & 50.8 & 67.3 & 33 & 47 & 64 & 36 & 54 & 70 \\
\hline & CGA & 36.4 & 50.8 & 66 & 34 & 47 & 63 & 38 & 53 & 71 \\
\hline & DIGA & 36.1 & 51.5 & 67.5 & 36 & 47 & 64 & 40 & 55 & 73 \\
\hline & PEGA & 40.6 & 56.4 & 73.3 & 38 & 51 & 66 & 40 & 59 & 77 \\
\hline & $\mathrm{PSO}+\mathrm{SA}$ & 38.6 & 54.4 & 70 & 36 & 51 & 65 & 40 & 57 & 75 \\
\hline \multirow[t]{8}{*}{ Case4 } & FABC & 24.1 & 36.1 & 50.9 & 25 & 34 & 49 & 24 & 38 & 55 \\
\hline & FDHS & 24.1 & 36.1 & 50.9 & 24 & 35 & 48 & 26 & 37 & 53 \\
\hline & EDA & 24.8 & 37.2 & 51.9 & 21 & 36 & 50 & 24 & 39 & 57 \\
\hline & HABC & 25.5 & 40 & 56.3 & 23 & 38 & 53 & 25 & 44 & 59 \\
\hline & CGA & 27.4 & 40.4 & 55 & 26 & 37 & 51 & 29 & 42 & 59 \\
\hline & DIGA & 29.6 & 42.4 & 56.9 & 28 & 40 & 56 & 30 & 46 & 63 \\
\hline & PEGA & 34.3 & 48.8 & 65.7 & 34 & 46 & 63 & 35 & 50 & 68 \\
\hline & $\mathrm{PSO}+\mathrm{SA}$ & 33.6 & 47.9 & 64.5 & 32 & 45 & 62 & 34 & 49 & 68 \\
\hline \multirow[t]{6}{*}{ Case5 } & FABC & 37.8 & 55.8 & 77.7 & 36 & 54 & 74 & 42 & 59 & 84 \\
\hline & FDHS & 37.9 & 55.8 & 77.8 & 36 & 54 & 74 & 42 & 59 & 84 \\
\hline & EDA & 38.6 & 56.9 & 78.3 & 36 & 55 & 73 & 40 & 60 & 81 \\
\hline & HABC & - & - & - & - & - & - & - & - & - \\
\hline & CGA & 47 & 65.4 & 86 & 42 & 62 & 82 & 49 & 70 & 91 \\
\hline & DIGA & 45.8 & 66.3 & 88.7 & 42 & 63 & 84 & 49 & 71 & 92 \\
\hline
\end{tabular}




\begin{tabular}{|c|c|c|c|c|c|c|c|c|c|c|}
\hline & PEGA & 50.3 & 74 & 96.5 & 48 & 68 & 94 & 50 & 74 & 100 \\
\hline & $\mathrm{PSO}+\mathrm{SA}$ & 51.2 & 74.6 & 97.6 & 48 & 72 & 93 & 52 & 73 & 101 \\
\hline \multirow[t]{4}{*}{ Case6 } & FABC & \multicolumn{3}{|c|}{52.50} & \multicolumn{3}{|c|}{55.83} & \multicolumn{3}{|c|}{ - } \\
\hline & FDHS & \multicolumn{3}{|c|}{52.40} & \multicolumn{3}{|c|}{55.94} & \multicolumn{3}{|c|}{ - } \\
\hline & HGTS & \multicolumn{3}{|c|}{50.25} & \multicolumn{3}{|c|}{51.50} & \multicolumn{3}{|c|}{ - } \\
\hline & SNSA & \multicolumn{3}{|c|}{63.75} & \multicolumn{3}{|c|}{65.65} & & & \\
\hline
\end{tabular}

Table 8 - 4 CPU time of eight algorithms for five cases

\begin{tabular}{|c|c|c|c|c|c|c|}
\hline Algorithm & Case1 & Case2 & Case3 & Case 4 & Case5 & Case6 \\
\hline $\mathrm{FABC}^{\mathrm{a}}$ & 1.00 & 1.00 & 1.35 & 1.37 & 2.41 & 2.46 \\
\hline FDHS $^{\text {a }}$ & 1.00 & 1.03 & 1.30 & 1.34 & 2.65 & 2.48 \\
\hline $\mathrm{EDA}^{\mathrm{b}}$ & 3.65 & 3.63 & 4.86 & 4.56 & 9.83 & - \\
\hline $\mathrm{HABC}^{\mathrm{c}}$ & 9.87 & 10.88 & 14.8 & 13.85 & - & - \\
\hline $\mathrm{CGA}^{\mathrm{d}}$ & 8.29 & 8.26 & 10.66 & 10.77 & 23.87 & - \\
\hline DIGA $^{\mathrm{d}}$ & 15.36 & 15.57 & 18.87 & 19.02 & 37.82 & - \\
\hline PEGA $^{\mathrm{d}}$ & 12.56 & 12.67 & 15.23 & 15.71 & 30.15 & - \\
\hline $\mathrm{PSO}+\mathrm{Sa}^{\mathrm{d}}$ & 12.4 & 12.33 & 15.24 & 15.66 & 30.9 & - \\
\hline HGTS ${ }^{\mathrm{e}}$ & - & - & - & - & - & 53.30 \\
\hline SNSA $^{\mathrm{f}}$ & - & - & - & - & - & 14.35 \\
\hline
\end{tabular}

To further test FABC algorithm, the eight instances in set two are solved. The average, best and worst values by FABC algorithm and six heuristics are shown in Table 8 - 5 . It can be seen from Table 8 - 5 that MinEnd heuristic obtains better results than other five heuristics. FABC can improve MinEnd obviously for all instances. FABC can improve the average fuzzy completion time results by MinEnd more than $30 \%$ for all instances. To show the scheduling results more clearly for eight instances in set two, Fig. 8 - 2 illustrates the best solution $(26,38,54)$ in fuzzy Gantt chart of instance Reman 2 for maximum fuzzy completion time objective. In addition, the average CPU times and generation for eight instances in set two are shown in Table 8 - 6. It can be seen from Table 8 - 6 that average CPU times for 1000 generations are less than 3.5 seconds for instance Reman 1 to Reman 4. For instance Reman 5 and Reman 6, the average CPU times are more than 30 seconds. The reason is that the instances become larger and the generation increases from 1000 to 4000. The instance sizes of Reman 7 and Reman 8 are 20-jobs, 10-machines, 308-operations and 20-jobs, 15-machines, 355-operations. The average CPU times are 82.99 seconds for Reman 7 and 102.77 seconds for Reman 8. Considering the results and average $\mathrm{CPU}$ times, FABC algorithm is effective for eight remanufacturing instances with maximum fuzzy completion time objective.

FABC algorithm is also compared to FDHS algorithm in Chapter 7 for solving eight instances from remanufacturing industry. FABC algorithm improves some measures' results by FDHS algorithm. For example, FABC obtains better results than those by FDHS for average and worst measures of Reman 
2. For Reman 4, FABC algorithm improves the average and best results by FDHS algorithm. FABC algorithm also improves the best, worst and best results by FDHS algorithm for Reman 5, Reman 6 and Reman 8, respectively. For Reman 1 to Reman 7, the average CPU times of FABC algorithm are larger than those of FDHS algorithm. FABC algorithm has smaller average CPU time than that of FDHS algorithm for Reman 8. However, the differences of average CPU times are not very obvious. To show the convergence of FABC algorithm, Fig. 8 - 3 shows the convergence curves of three ranking criteria results by FABC for case 5 and Reman 8. For the same value of the first ranking criterion, the values of the second and the third ranking criteria may become larger or smaller to show the change of results. For the same values of the first and second ranking criteria, the third ranking criterion may become larger or smaller to present results changes. Hence, the first ranking criterion is non-increasing for two instances. The second and third ranking criteria may increase or decrease while the third ranking criterion has larger fluctuations than the second one. In summary, FABC algorithm is effective for fuzzy maximum completion time objective of FJSSP with fuzzy processing time. 


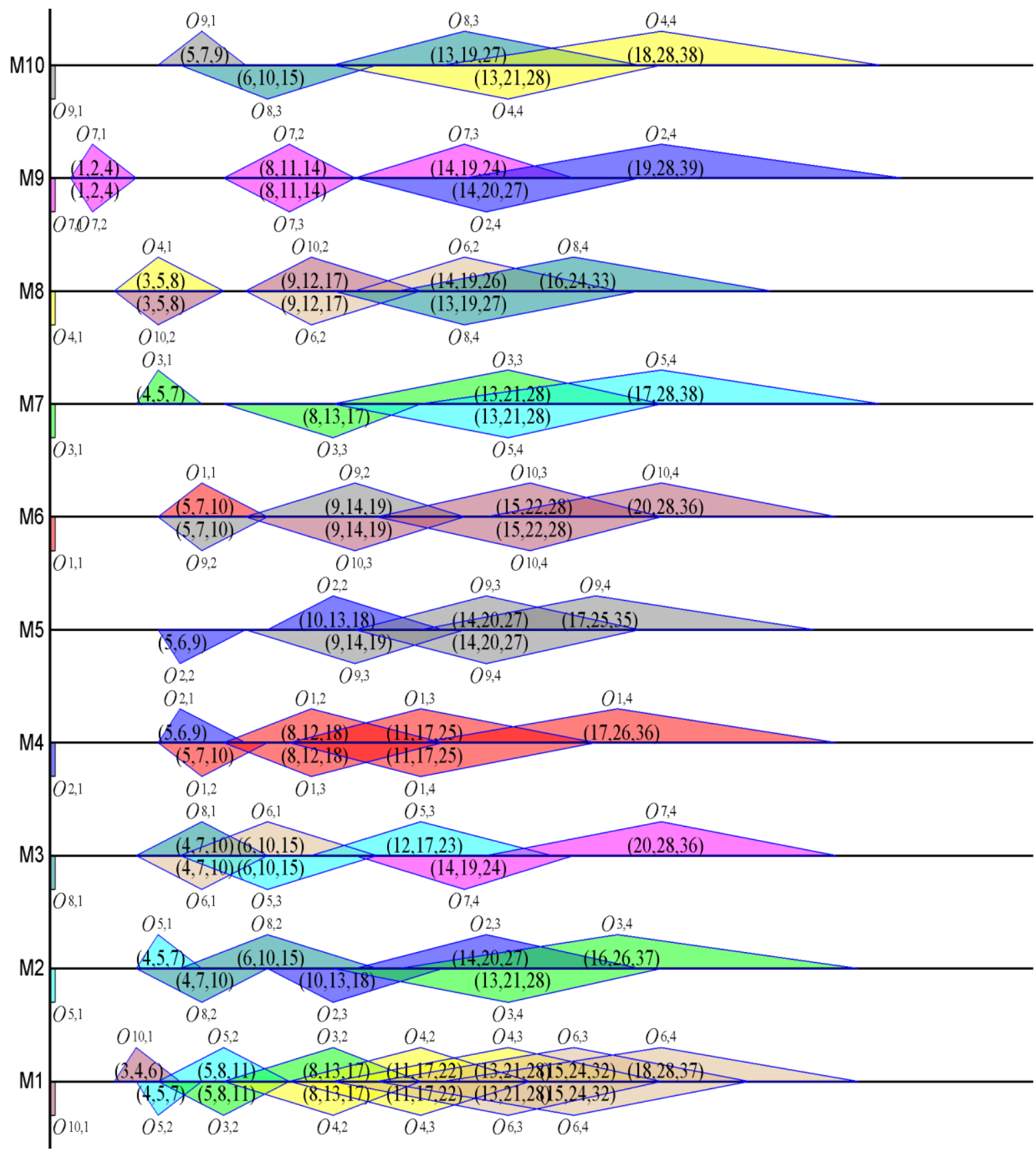

Fig. 8 - 1 Best maximum fuzzy completion time Gantt chart of case 1 by FABC

Table 8 - 5 Maximum fuzzy completion time for eight Reman instances

\begin{tabular}{|c|c|c|c|c|c|c|c|c|c|c|}
\hline Instance & Algorithm & \multicolumn{3}{|c|}{ Average value } & \multicolumn{3}{|c|}{ Best value } & \multicolumn{3}{|c|}{ Worst value } \\
\hline \multirow{3}{*}{ Reman 1} & $\mathrm{FABC}$ & 17.6 & 26.0 & 34.6 & 19 & 26 & 33 & 19 & 26 & 36 \\
\hline & FDHS & 17.4 & 26.0 & 34.7 & 19 & 26 & 33 & 18 & 26 & 36 \\
\hline & MinEnd & 25.1 & 38.9 & 51.8 & 18 & 30 & 42 & 37 & 52 & 68 \\
\hline
\end{tabular}




\begin{tabular}{|c|c|c|c|c|c|c|c|c|c|c|}
\hline & GS & 31.7 & 47.5 & 63.2 & 25 & 36 & 48 & 41 & 61 & 81 \\
\hline & LS & 29.0 & 46.6 & 61.2 & 17 & 34 & 45 & 40 & 61 & 79 \\
\hline & MReW & 29.6 & 47.3 & 64.7 & 20 & 33 & 45 & 58 & 80 & 107 \\
\hline & $\mathrm{MReO}$ & 28.9 & 41.8 & 55.9 & 16 & 30 & 42 & 57 & 76 & 96 \\
\hline & Random & 33.4 & 50.9 & 67.8 & 14 & 30 & 42 & 74 & 96 & 124 \\
\hline \multirow{8}{*}{ Reman 2} & FABC & 24.9 & 40.6 & 56.7 & 26 & 38 & 54 & 26 & 43 & 60 \\
\hline & FDHS & 24.7 & 40.7 & 56.8 & 17 & 39 & 58 & 28 & 43 & 59 \\
\hline & MinEnd & 37.5 & 61.2 & 84.9 & 31 & 51 & 73 & 48 & 72 & 96 \\
\hline & GS & 53.6 & 83.8 & 116.3 & 44 & 66 & 91 & 73 & 116 & 160 \\
\hline & LS & 59.3 & 95.2 & 134.3 & 47 & 78 & 108 & 69 & 109 & 153 \\
\hline & MReW & 69.1 & 106.7 & 147.2 & 48 & 75 & 107 & 119 & 167 & 228 \\
\hline & $\mathrm{MReO}$ & 49.9 & 75.5 & 104.8 & 37 & 59 & 80 & 77 & 100 & 140 \\
\hline & Random & 71.6 & 108.6 & 148.7 & 39 & 65 & 97 & 122 & 169 & 223 \\
\hline \multirow[t]{8}{*}{ Reman 3} & FABC & 37.6 & 59.6 & 80.8 & 37 & 59 & 76 & 46 & 61 & 83 \\
\hline & FDHS & 36.8 & 59.1 & 80.3 & 37 & 58 & 77 & 43 & 60 & 79 \\
\hline & MinEnd & 64.8 & 99.7 & 133.2 & 57 & 89 & 115 & 69 & 110 & 144 \\
\hline & GS & 76.7 & 117.7 & 159.5 & 56 & 91 & 120 & 91 & 133 & 179 \\
\hline & LS & 82.9 & 128.7 & 173.9 & 67 & 101 & 135 & 108 & 160 & 210 \\
\hline & MReW & 94.9 & 144.6 & 194.0 & 61 & 102 & 138 & 142 & 198 & 262 \\
\hline & $\mathrm{MReO}$ & 69.7 & 104.4 & 137.2 & 47 & 83 & 116 & 115 & 146 & 185 \\
\hline & Random & 93.1 & 142.8 & 191.3 & 61 & 99 & 132 & 165 & 223 & 289 \\
\hline \multirow[t]{8}{*}{ Reman 4} & FABC & 33.6 & 50.9 & 70.3 & 34 & 49 & 67 & 35 & 54 & 75 \\
\hline & FDHS & 34.3 & 51.0 & 70.2 & 31 & 49 & 70 & 35 & 54 & 75 \\
\hline & MinEnd & 53.2 & 81.0 & 111.7 & 38 & 67 & 94 & 63 & 104 & 147 \\
\hline & GS & 67.2 & 103.9 & 141.7 & 58 & 91 & 126 & 94 & 131 & 176 \\
\hline & LS & 74.0 & 118.9 & 166.4 & 55 & 101 & 144 & 91 & 142 & 198 \\
\hline & MReW & 91.0 & 140.0 & 192.6 & 56 & 102 & 143 & 146 & 199 & 275 \\
\hline & $\mathrm{MReO}$ & 64.4 & 95.0 & 129.6 & 48 & 77 & 106 & 112 & 147 & 188 \\
\hline & Random & 92.7 & 141.5 & 193.5 & 60 & 100 & 139 & 160 & 222 & 301 \\
\hline \multirow[t]{8}{*}{ Reman 5} & FABC & 52.3 & 84.5 & 116.3 & 44 & 82 & 119 & 59 & 88 & 116 \\
\hline & FDHS & 52.6 & 84.4 & 116.1 & 53 & 82 & 112 & 55 & 86 & 121 \\
\hline & MinEnd & 88.1 & 140.6 & 193.6 & 82 & 132 & 184 & 103 & 150 & 206 \\
\hline & GS & 110.6 & 172.4 & 234.8 & 100 & 151 & 205 & 138 & 209 & 273 \\
\hline & LS & 143.5 & 217.5 & 291.4 & 120 & 182 & 242 & 166 & 248 & 334 \\
\hline & MReW & 147.6 & 234.8 & 317.4 & 111 & 173 & 230 & 237 & 324 & 418 \\
\hline & $\mathrm{MReO}$ & 106.2 & 165.2 & 223.9 & 88 & 139 & 194 & 166 & 233 & 314 \\
\hline & Random & 141.5 & 224.8 & 305.3 & 103 & 181 & 250 & 227 & 317 & 416 \\
\hline \multirow[t]{8}{*}{ Reman 6} & FABC & 44.0 & 72.8 & 98.9 & 39 & 71 & 98 & 44 & 75 & 104 \\
\hline & FDHS & 44.5 & 72.6 & 98.5 & 44 & 69 & 94 & 52 & 76 & 98 \\
\hline & MinEnd & 70.8 & 118.7 & 162.0 & 62 & 100 & 139 & 67 & 134 & 186 \\
\hline & GS & 96.0 & 156.1 & 213.9 & 84 & 133 & 173 & 108 & 178 & 246 \\
\hline & LS & 109.6 & 178.0 & 243.3 & 83 & 154 & 215 & 136 & 214 & 293 \\
\hline & MReW & 142.2 & 223.1 & 302.8 & 94 & 162 & 219 & 219 & 313 & 415 \\
\hline & $\mathrm{MReO}$ & 102.8 & 153.5 & 204.8 & 66 & 114 & 153 & 162 & 210 & 271 \\
\hline & Random & 133.2 & 212.2 & 288.0 & 86 & 162 & 221 & 232 & 314 & 411 \\
\hline
\end{tabular}




\begin{tabular}{llccccccccc}
\hline \multirow{2}{*}{ Reman7 } & FABC & $\mathbf{6 1 . 0}$ & $\mathbf{1 0 7 . 9}$ & $\mathbf{1 5 3 . 2}$ & $\mathbf{6 3}$ & $\mathbf{1 0 5}$ & $\mathbf{1 4 8}$ & $\mathbf{6 0}$ & $\mathbf{1 1 3}$ & $\mathbf{1 5 7}$ \\
& & 60.4 & 107.7 & 153.3 & 60 & 105 & 149 & 58 & 112 & 159 \\
& MinEnd & 96.9 & 167.2 & 233.6 & 94 & 152 & 214 & 100 & 179 & 254 \\
& GS & 132.9 & 228.4 & 315.7 & 112 & 205 & 286 & 153 & 255 & 354 \\
& LS & 149.8 & 265.4 & 368.5 & 136 & 236 & 330 & 169 & 297 & 414 \\
& MReW & 189.8 & 322.5 & 446.9 & 134 & 258 & 366 & 329 & 471 & 631 \\
& MReO & 159.1 & 248.1 & 336.0 & 100 & 177 & 248 & 231 & 342 & 446 \\
& Random & 200.6 & 331.1 & 453.7 & 139 & 250 & 351 & 351 & 492 & 646 \\
& & & & & & & & & & \\
Reman8 & FABC & $\mathbf{5 7 . 8}$ & $\mathbf{9 3 . 5}$ & $\mathbf{1 2 7 . 0}$ & $\mathbf{5 3}$ & $\mathbf{8 8}$ & $\mathbf{1 2 2}$ & $\mathbf{6 3}$ & $\mathbf{9 6}$ & $\mathbf{1 3 3}$ \\
& FDHS & 57.7 & 92.3 & 126.6 & 56 & 89 & 122 & 57 & 95 & 133 \\
& MinEnd & 84.9 & 141.1 & 192.8 & 83 & 132 & 172 & 95 & 153 & 206 \\
& GS & 120.2 & 202.2 & 278.0 & 102 & 178 & 251 & 139 & 231 & 317 \\
& LS & 143.5 & 239.1 & 329.8 & 131 & 207 & 282 & 173 & 284 & 390 \\
& MReW & 228.9 & 358.1 & 485.7 & 152 & 274 & 374 & 326 & 443 & 581 \\
& MReO & 130.2 & 204.9 & 279.3 & 103 & 172 & 231 & 199 & 274 & 367 \\
& Random & 208.9 & 331.9 & 451.7 & 134 & 240 & 330 & 350 & 482 & 643 \\
\hline
\end{tabular}

Table 8 - 6 CPU time and generation for maximum fuzzy completion time

\begin{tabular}{|c|c|c|c|c|c|c|c|}
\hline \multirow{2}{*}{ Instance } & \multicolumn{2}{|c|}{ CPU time } & \multirow{2}{*}{ Generation } & \multirow{2}{*}{ Instance } & \multicolumn{2}{|c|}{ Cpu time } & \multirow{2}{*}{ Generation } \\
\hline & FABC & FDHS & & & FABC & FDHS & \\
\hline Reman 1 & 0.44 & 0.40 & 1000 & Reman 5 & 30.45 & 22.38 & 4000 \\
\hline Reman 2 & 1.72 & 1.44 & 1000 & Reman 6 & 33.75 & 25.97 & 4000 \\
\hline Reman 3 & 2.29 & 1.89 & 1000 & Reman7 & 82.99 & 73.83 & 4000 \\
\hline Reman 4 & 3.23 & 2.68 & 1000 & Reman8 & 102.77 & 103.56 & 4000 \\
\hline
\end{tabular}

Notes: 2.40GHz CPU

\subsubsection{Maximum fuzzy machine workload objective}

In both scheduling and rescheduling stages, we calculated the maximum machine workload for current scheduling stage and non-for summary of all scheduling and rescheduling stages. For maximum fuzzy machine workload objective, we compared FABC algorithm to six heuristics, namely MinEnd, GS, LS, MReW, MaxReO and random rule. To show FABC algorithm performance, the following formula is employed:

$$
\operatorname{Imp}(i)=\frac{\left(t_{1}^{i}+2 t_{2}^{i}+t_{3}^{i}\right) / 4-\left(t_{1}^{0}+2 t_{2}^{0}+t_{3}^{0}\right) / 4}{\left(t_{1}^{0}+2 t_{2}^{0}+t_{3}^{0}\right) / 4} \times 100 \%
$$

where $t_{1}^{i}, t_{2}^{i}$ and $t_{3}^{i}$ are the results of $i^{\text {th }}$ heuristic represented by TFN; $t_{1}^{0}, t_{2}^{0}$ and $t_{3}^{0}$ are the result of FABC algorithm represented by TFN. $\left(t_{1}^{i}+2 t_{2}^{i}+t_{3}^{i}\right) / 4$ is the first criterion to compare two TFNs. Since the results by FABC algorithm are better than those by six heuristics, the first criterion is enough to compare them. It is clear that the larger the $\operatorname{Imp}(i)$ is, FABC algorithm is more competitive than $i^{\text {th }}$ heuristic. For five cases in set one, the maximum fuzzy machine workload results of FABC algorithm and $\operatorname{Imp}(i)$ values of six heuristics are shown in Table 8 - 7. It can be seen from Table 8 - 7 
that the average values by six heuristics are larger than those obtained by FABC by at least $10.6 \%$ for five cases. The best values and worst values by six heuristics are larger than those obtained by FABC by at least $4.6 \%$ and $14 \%$ for five cases, respectively.

For eight instances from remanufacturing, the maximum fuzzy machine workload results of FABC and six heuristics are evaluated. The $\operatorname{Imp}(i)$ results of six heuristics to FABC algorithm are also computed. All the results are shown in Table 8 - 8. It can be seen from Table 8 - 8 that FABC algorithm can improve six heuristics average, best and worst values. For instance, Reman 1, FABC finds the same result in 30 runs because this instance is a small-scale problem with 5-jobs, 4-machines and 23operations. The average values of six heuristics are larger than those obtained by FABC by at least 16.7\%. The best values of six heuristics are larger than those of FABC by at least $8.0 \%$. Except for instance Reman 1, the best values of six heuristics are larger than those of FABC by at least $14.4 \%$. The worst values of six heuristics are larger than those of FABC by at least $20.3 \%$.

To show the maximum machine workload results of FABC clearly, Fig. 8 - 4 and Fig. 8 - 5 show the best fuzzy Gantt charts of case 1 and instance Reman 2. The maximum fuzzy machine workload of case 1 is $(17,25,35)$ in the best solution while the maximum fuzzy machine workload of instance Reman 2 is $(13,30,41)$. In addition, the CPU times and generations of FABC algorithm are shown in Table 8 - 9. For five cases in set one and the previous four instances in set two, the generations are 1000 and the CPU times are less than 3.5 seconds. For the last four instances in set two, the generations are 4000 and the CPU times are 25.23, 30.07, 66.65 and 107.97 seconds respectively. With respect to the scale of problems, the CPU time is very short for all thirteen problems.

To show the convergence of FABC for maximum fuzzy machine workload objective, Fig. 8 - 6 presents the curves of three ranking criteria results by FABC for case 5 and Reman 8. Similar to the convergence of maximum fuzzy completion time, the first ranking criterion is non-increasing for two instances. The second and third ranking criteria may increase or decrease while the third ranking criterion has larger fluctuations than the second one. In summary, FABC algorithm has good performance for fuzzy maximum machine workload objective for FJSSP with fuzzy processing time. 


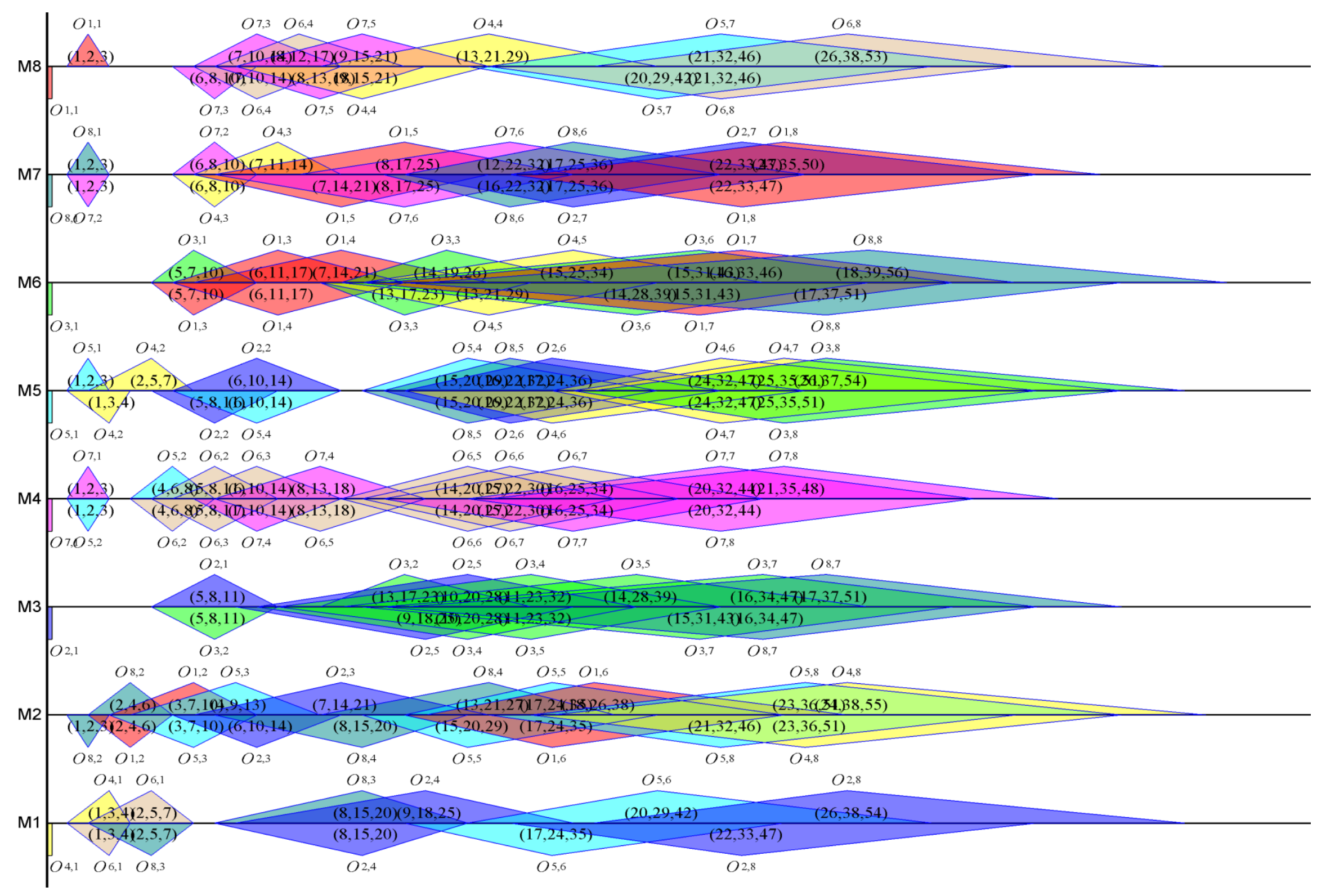

Fig. 8 - 2 Best maximum fuzzy completion time Gantt chart of Reman 2 by FABC 

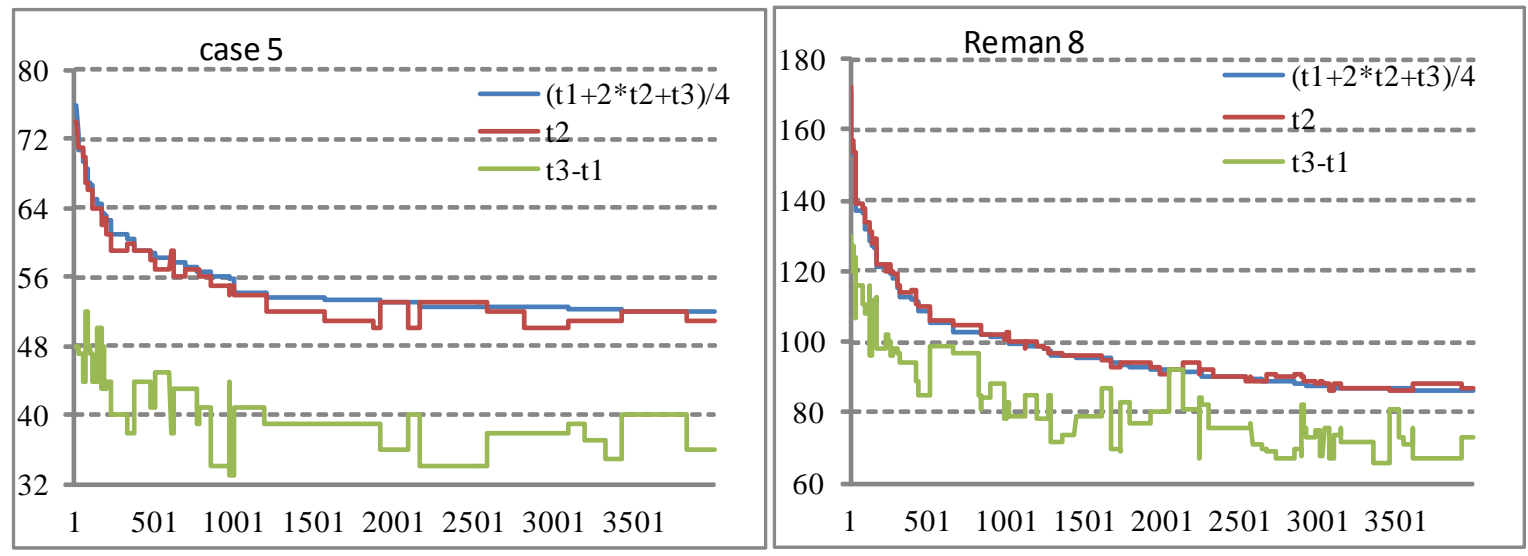

Fig. 8 - 3 Maximum fuzzy completion time convergence curves of case 5 and Reman 8

Table 8 - 7 Maximum fuzzy machine workload by EABC and improvement to six heuristics

\begin{tabular}{|c|c|c|c|c|c|c|c|c|c|c|c|c|c|}
\hline \multirow{2}{*}{$\begin{array}{l}\text { Instance } \\
\text { Case1 }\end{array}$} & \multirow{2}{*}{$\begin{array}{l}\text { Algorithm } \\
\text { FABC }\end{array}$} & \multicolumn{3}{|c|}{ Average value } & \multirow[t]{2}{*}{$\%$} & \multicolumn{3}{|c|}{ Best value } & \multirow[t]{2}{*}{$\%$} & \multicolumn{3}{|c|}{ Worst value } & \multirow[t]{2}{*}{$\%$} \\
\hline & & 17.3 & 25.6 & 35.4 & & 17 & 25 & 35 & & 19 & 26 & 36 & \\
\hline & MinEnd & & & & 32.1 & & & & 18.6 & & & & 51.4 \\
\hline & GS & & & & 18.4 & & & & 10.8 & & & & 25.2 \\
\hline & LS & & & & 89.6 & & & & 93.1 & & & & 84.1 \\
\hline & MReW & & & & 152.6 & & & & 71.6 & & & & 240.2 \\
\hline & $\mathrm{MReO}$ & & & & 139.5 & & & & 60.8 & & & & 274.8 \\
\hline & Random & & & & 130.5 & & & & 52.9 & & & & 206.5 \\
\hline \multirow[t]{7}{*}{ Case2 } & FABC & 27.7 & 38.2 & 49.7 & & 29 & 38 & 46 & & 28 & 39 & 50 & \\
\hline & MinEnd & & & & 31.4 & & & & 11.3 & & & & 59.0 \\
\hline & GS & & & & 16.1 & & & & 4.6 & & & & 23.1 \\
\hline & LS & & & & 95.1 & & & & 98.7 & & & & 92.3 \\
\hline & MReW & & & & 119.4 & & & & 55.0 & & & & 234.0 \\
\hline & $\mathrm{MReO}$ & & & & 147.3 & & & & 54.3 & & & & 278.8 \\
\hline & Random & & & & 131.9 & & & & 58.9 & & & & 230.8 \\
\hline \multirow[t]{7}{*}{ Case 3} & FABC & 27.8 & 39.9 & 52.3 & & 28 & 38 & 51 & & 27 & 40 & 57 & \\
\hline & MinEnd & & & & 28.7 & & & & 7.7 & & & & 46.3 \\
\hline & GS & & & & 13.5 & & & & 12.9 & & & & 20.7 \\
\hline & LS & & & & 76.4 & & & & 81.9 & & & & 72.0 \\
\hline & MReW & & & & 123.0 & & & & 71.0 & & & & 215.2 \\
\hline & $\mathrm{MReO}$ & & & & 137.2 & & & & 49.7 & & & & 222.6 \\
\hline & Random & & & & 117.4 & & & & 60.0 & & & & 201.8 \\
\hline \multirow[t]{7}{*}{ Case4 } & FABC & 19.2 & 29.5 & 42.3 & & 17 & 29 & 43 & & 19 & 30 & 44 & \\
\hline & MinEnd & & & & 34.6 & & & & 18.6 & & & & 52.8 \\
\hline & GS & & & & 17.9 & & & & 13.6 & & & & 26.8 \\
\hline & $\mathrm{LS}$ & & & & 32.8 & & & & 35.6 & & & & 30.1 \\
\hline & MReW & & & & 125.5 & & & & 68.6 & & & & 226.0 \\
\hline & $\mathrm{MReO}$ & & & & 120.9 & & & & 45.8 & & & & 225.2 \\
\hline & Random & & & & 103.1 & & & & 48.3 & & & & 173.2 \\
\hline
\end{tabular}




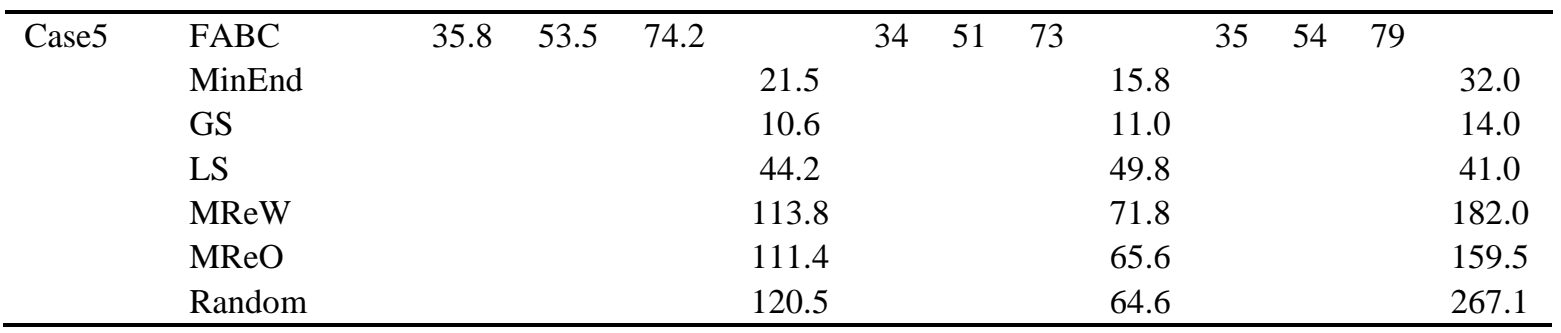

Table 8 - 8 Maximum fuzzy machine workload results for eight Reman instances

\begin{tabular}{|c|c|c|c|c|c|c|c|c|c|c|c|c|c|}
\hline \multirow{2}{*}{$\frac{\text { Instance }}{\text { Reman } 1}$} & \multirow{2}{*}{$\begin{array}{l}\text { Algorithm } \\
\text { FABC }\end{array}$} & \multicolumn{3}{|c|}{ Average value } & \multirow[t]{2}{*}{$\%$} & \multicolumn{3}{|c|}{ Best value } & \multirow[t]{2}{*}{$\%$} & \multicolumn{3}{|c|}{ Worst value } & \multirow[t]{2}{*}{$\%$} \\
\hline & & 13 & 22 & 30 & & 13 & 22 & 30 & & 13 & 22 & 30 & \\
\hline & MinEnd & 19.2 & 29.6 & 39.8 & 35.9 & 13 & 24 & 33 & 8.0 & 29 & 39 & 55 & 86.2 \\
\hline & GS & 16.6 & 27.2 & 36.9 & 24.0 & 13 & 24 & 33 & 8.0 & 20 & 32 & 45 & 48.3 \\
\hline & LS & 15 & 28 & 39 & 26.4 & 15 & 28 & 39 & 26.4 & 15 & 28 & 39 & 26.4 \\
\hline & MReW & 22.6 & 34.9 & 47 & 60.2 & 14 & 24 & 33 & 9.2 & 46 & 64 & 82 & 194.3 \\
\hline & $\mathrm{MReO}$ & 21 & 33.1 & 44.8 & 51.7 & 14 & 24 & 33 & 9.2 & 41 & 55 & 71 & 155.2 \\
\hline & Random & 21.6 & 33.7 & 45.1 & 54.1 & 15 & 26 & 37 & 19.5 & 40 & 55 & 71 & 154.0 \\
\hline \multirow[t]{7}{*}{ Reman 2} & FABC & 16.5 & 29.5 & 41.1 & & 13 & 30 & 41 & & 17 & 30 & 42 & \\
\hline & MinEnd & 27.8 & 44.4 & 60.3 & 51.7 & 23 & 38 & 51 & 31.6 & 38 & 56 & 80 & 93.3 \\
\hline & GS & 23.6 & 37.9 & 52.3 & 30.1 & 20 & 34 & 48 & 19.3 & 31 & 46 & 65 & 58.0 \\
\hline & LS & 31 & 45 & 65 & 59.5 & 31 & 45 & 65 & 63.2 & 31 & 45 & 65 & 56.3 \\
\hline & MReW & 32.1 & 52 & 71.1 & 77.7 & 24 & 39 & 53 & 36.0 & 58 & 86 & 111 & 186.6 \\
\hline & $\mathrm{MReO}$ & 33.6 & 53.5 & 73.8 & 83.9 & 26 & 41 & 54 & 42.1 & 63 & 88 & 120 & 201.7 \\
\hline & Random & 33.5 & 53.2 & 73.2 & 82.8 & 27 & 39 & 55 & 40.4 & 57 & 78 & 109 & 170.6 \\
\hline \multirow[t]{7}{*}{ Reman 3} & FABC & 32.3 & 53.3 & 73.1 & & 34 & 53 & 71 & & 36 & 54 & 70 & \\
\hline & MinEnd & 45.7 & 72.2 & 97 & 35.4 & 41 & 63 & 82 & 18.0 & 53 & 82 & 111 & 53.3 \\
\hline & GS & 42.8 & 67.9 & 91.3 & 27.3 & 37 & 61 & 88 & 17.1 & 50 & 75 & 99 & 39.7 \\
\hline & LS & 51 & 76 & 102 & 43.9 & 51 & 76 & 102 & 44.5 & 51 & 76 & 102 & 42.5 \\
\hline & MReW & 56.4 & 86.6 & 114.8 & 62.5 & 40 & 64 & 85 & 19.9 & 96 & 129 & 167 & 143.5 \\
\hline & $\mathrm{MReO}$ & 58.2 & 90.4 & 121.1 & 69.9 & 33 & 62 & 88 & 16.1 & 93 & 132 & 174 & 148.1 \\
\hline & Random & 56.5 & 87.2 & 116.7 & 64.0 & 43 & 68 & 91 & 28.0 & 91 & 126 & 165 & 137.4 \\
\hline \multirow[t]{7}{*}{ Reman 4} & FABC & 21.4 & 37.5 & 53.1 & & 28 & 36 & 48 & & 22 & 37 & 56 & \\
\hline & MinEnd & 36 & 53.2 & 73.5 & 44.4 & 33 & 46 & 65 & 28.4 & 50 & 67 & 89 & 79.6 \\
\hline & GS & 36.9 & 50.9 & 68.8 & 38.8 & 29 & 45 & 60 & 20.9 & 42 & 60 & 85 & 62.5 \\
\hline & LS & 46 & 72 & 103 & 96.0 & 46 & 72 & 103 & 98.0 & 46 & 72 & 103 & 92.8 \\
\hline & MReW & 49.9 & 73.2 & 99.7 & 98.0 & 39 & 58 & 81 & 59.5 & 89 & 111 & 146 & 200.7 \\
\hline & $\mathrm{MReO}$ & 55.9 & 80.9 & 109.5 & 118.9 & 42 & 59 & 79 & 61.5 & 76 & 108 & 145 & 187.5 \\
\hline & Random & 52.2 & 74.2 & 100.9 & 101.7 & 40 & 56 & 76 & 54.1 & 73 & 103 & 143 & 177.6 \\
\hline Reman 5 & FABC & 43.5 & 74.4 & 104.9 & & 36 & 75 & 109 & & 43 & 74 & 108 & \\
\hline
\end{tabular}




\begin{tabular}{|c|c|c|c|c|c|c|c|c|c|c|c|c|c|}
\hline & MinEnd & 64.7 & 103.7 & 141.5 & 39.2 & 53 & 95 & 128 & 25.8 & 74 & 121 & 165 & 60.9 \\
\hline & GS & 55.6 & 89.5 & 122.5 & 20.2 & 50 & 84 & 120 & 14.6 & 60 & 96 & 130 & 27.8 \\
\hline & LS & 72 & 101 & 137 & 38.3 & 72 & 101 & 137 & 39.3 & 72 & 101 & 137 & 37.5 \\
\hline & MReW & 89.2 & 138 & 186.3 & 85.6 & 67 & 104 & 139 & 40.3 & 128 & 183 & 244 & 146.8 \\
\hline & $\mathrm{MReO}$ & 95 & 146.1 & 196.1 & 96.3 & 67 & 105 & 144 & 42.7 & 149 & 208 & 273 & 180.3 \\
\hline & Random & 83.4 & 134.3 & 182.4 & 79.8 & 61 & 101 & 135 & 34.9 & 132 & 190 & 255 & 156.5 \\
\hline \multirow[t]{7}{*}{ Reman 6} & FABC & 34.5 & 62 & 86.2 & & 30 & 62 & 87 & & 38 & 63 & 85 & \\
\hline & MinEnd & 52.8 & 87.4 & 119 & 41.6 & 45 & 77 & 103 & 25.3 & 63 & 100 & 140 & 61.8 \\
\hline & GS & 45.7 & 76.2 & 104.1 & 23.5 & 41 & 71 & 100 & 17.4 & 54 & 84 & 111 & 33.7 \\
\hline & LS & 56 & 88 & 119 & 43.4 & 56 & 88 & 119 & 45.6 & 56 & 88 & 119 & 41.0 \\
\hline & MReW & 77.4 & 118 & 159.4 & 93.2 & 52 & 84 & 115 & 39.0 & 119 & 173 & 229 & 178.7 \\
\hline & $\mathrm{MReO}$ & 69.1 & 109 & 148.5 & 78.0 & 46 & 79 & 107 & 29.0 & 136 & 186 & 250 & 204.4 \\
\hline & Random & 64.9 & 105.6 & 143.4 & 71.4 & 50 & 85 & 119 & 40.7 & 127 & 178 & 241 & 190.8 \\
\hline \multirow[t]{7}{*}{ Reman7 } & FABC & 50.5 & 97 & 138.5 & & 49 & 97 & 137 & & 50 & 97 & 145 & \\
\hline & MinEnd & 75.5 & 129.4 & 181.6 & 34.7 & 63 & 116 & 166 & 21.3 & 90 & 144 & 196 & 47.6 \\
\hline & GS & 67.7 & 115.6 & 162.2 & 20.4 & 65 & 111 & 158 & 17.1 & 76 & 122 & 166 & 24.9 \\
\hline & LS & 66 & 122 & 172 & 25.8 & 66 & 122 & 172 & 26.8 & 66 & 122 & 172 & 23.9 \\
\hline & MReW & 115.8 & 188.8 & 259.2 & 96.5 & 79 & 146 & 198 & 49.7 & 179 & 251 & 340 & 162.5 \\
\hline & $\mathrm{MReO}$ & 107.8 & 181.5 & 249.2 & 88.0 & 77 & 137 & 195 & 43.7 & 169 & 263 & 354 & 169.7 \\
\hline & Random & 101.4 & 174.4 & 240.5 & 80.3 & 78 & 139 & 200 & 46.3 & 157 & 233 & 313 & 140.6 \\
\hline \multirow[t]{7}{*}{ Reman8 } & FABC & 40.6 & 75.3 & 106.8 & & 42 & 74 & 101 & & 44 & 77 & 107 & \\
\hline & MinEnd & 59.6 & 100.3 & 138.9 & 33.9 & 52 & 91 & 129 & 24.7 & 71 & 120 & 163 & 55.4 \\
\hline & GS & 50 & 88.1 & 121.5 & 16.7 & 42 & 85 & 121 & 14.4 & 49 & 95 & 128 & 20.3 \\
\hline & LS & 62 & 101 & 139 & 35.2 & 62 & 101 & 139 & 38.5 & 62 & 101 & 139 & 32.1 \\
\hline & MReW & 90.8 & 151.1 & 207.3 & 101.4 & 61 & 110 & 156 & 50.2 & 158 & 215 & 286 & 186.6 \\
\hline & $\mathrm{MReO}$ & 81.4 & 140 & 192.4 & 85.8 & 51 & 110 & 161 & 48.5 & 139 & 200 & 266 & 163.9 \\
\hline & Random & 93.6 & 152.7 & 206.8 & 103.3 & 55 & 108 & 149 & 44.3 & 154 & 227 & 301 & 198.0 \\
\hline
\end{tabular}

Table 8 - 9 Average CPU time and generation for maximum fuzzy machine workload objective

\begin{tabular}{llllll}
\hline Instance & CPU time & Generation & Instance & CPU time & Generation \\
\hline case 1 & 1.01 & 1000 & Reman 3 & 2.24 & 1000 \\
case 2 & 1.01 & 1000 & Reman 4 & 3.32 & 1000 \\
case 3 & 1.37 & 1000 & Reman 5 & 25.23 & 4000 \\
case 4 & 1.36 & 1000 & Reman 6 & 30.07 & 4000 \\
case 5 & 2.59 & 1000 & Reman7 & 66.65 & 4000 \\
Reman 1 & 0.48 & 1000 & Reman8 & 107.97 & 4000 \\
Reman 2 & 1.76 & 1000 & & & \\
\hline
\end{tabular}




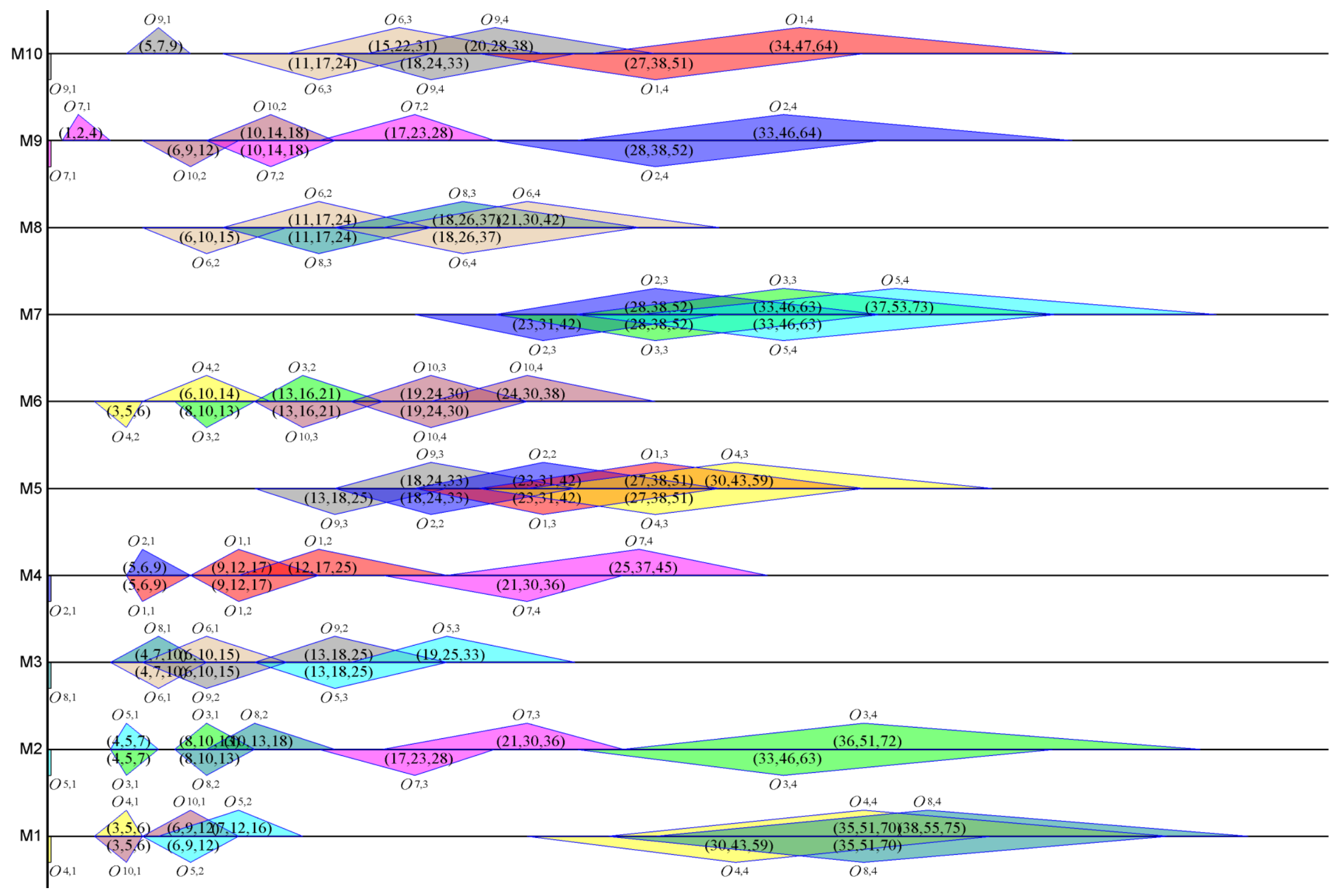

Fig. 8 - 4 Best maximum fuzzy machine workload Gantt chart of case 1 by FABC 


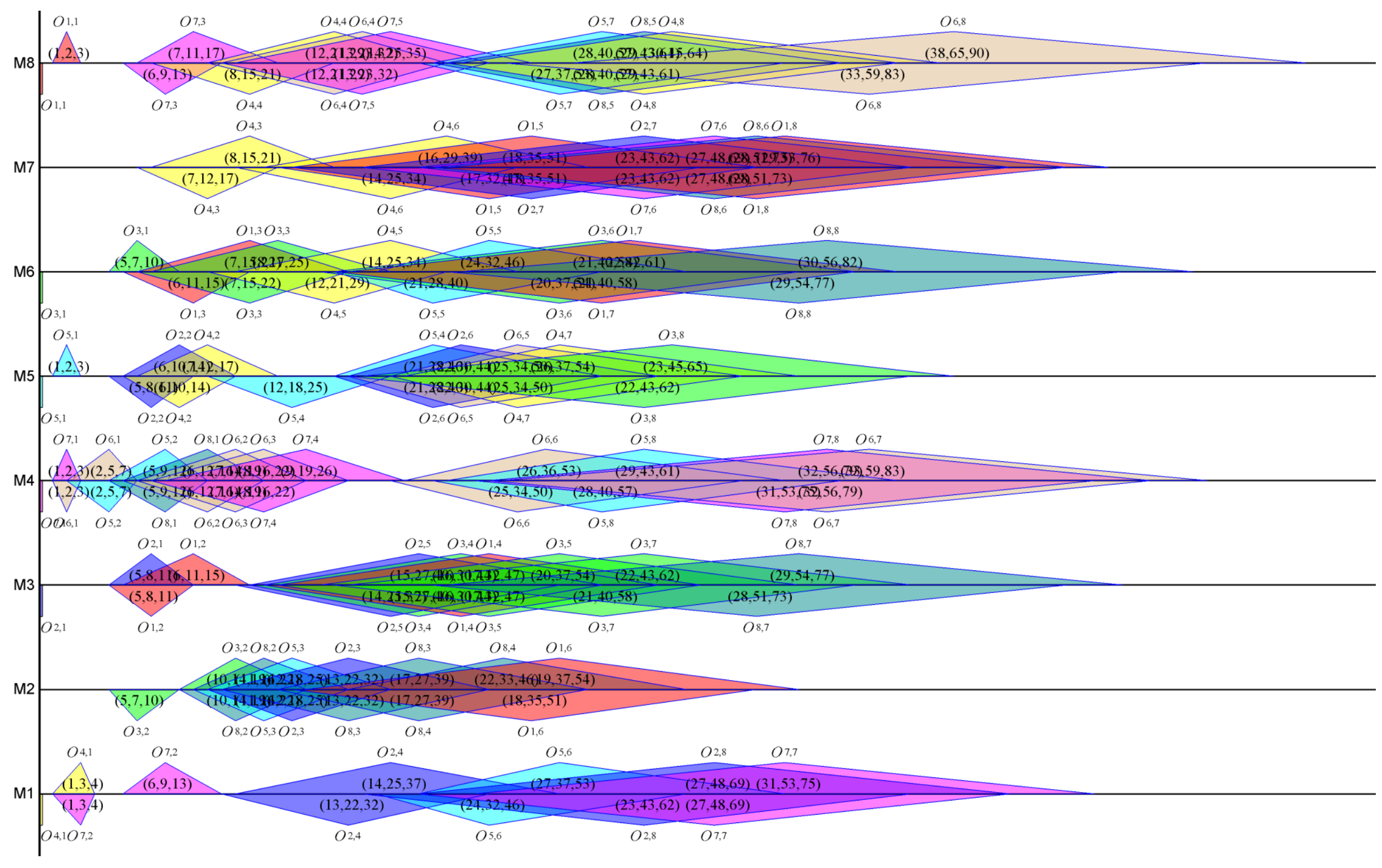

Fig. 8 - 5 Best maximum fuzzy machine workload Gantt chart of Reman 2 by FABC 


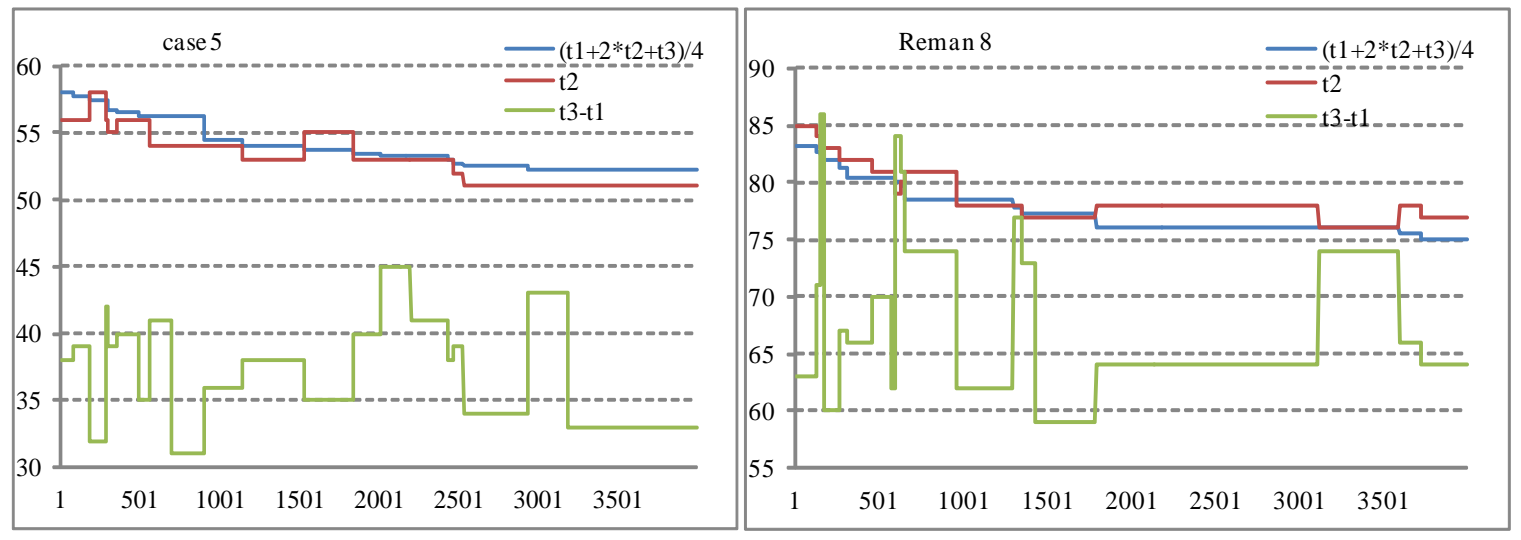

Fig. 8 - 6 Maximum fuzzy machine workload convergence curves of case 5 and Reman 8

\subsection{Conclusions}

In this Chapter, an effective fuzzy artificial bee colony (FABC) algorithm was proposed to solve flexible job shop scheduling problem with fuzzy processing time. The objectives were to minimize the maximum fuzzy completion time and the maximum fuzzy machine workload, respectively. The MinEnd heuristic was evaluated by comparing with five simple heuristics for maximum fuzzy completion time and maximum fuzzy machine workload objectives. FABC was compared to six existing algorithms for solving five benchmark cases. The results and comparisons showed the effectiveness of FABC. Eight instances from remanufacturing were solved by FABC algorithm and six heuristics for maximum fuzzy completion time objective. FABC improved the results of heuristics in short CPU time. For maximum fuzzy machine workload objective, five benchmark cases and eight remanufacturing instances were solved by FABC and six heuristics. The results and comparisons demonstrate improved performance of FABC algorithm. The experiments also investigated the convergence of FABC algorithm. Several convergence curves were shown. In addition, several fuzzy Gantt charts of best solution were shown for maximum fuzzy completion time and maximum fuzzy machine workload objectives. 


\section{Chapter 9}

\section{Two-stage FABC Algorithm for Multi- objective and Multi-constraint FJSSP}

\subsection{Introduction}

This Chapter researches multi-objective multi-constraints flexible job shop scheduling problem (FJSSP). The constraints are fuzzy processing time and new job insertion. These two constraints are modeled from remanufacturing. The objectives are to minimize the maximum fuzzy completion time and the maximum fuzzy machine workload. Based on the fuzzy artificial bee colony algorithm (FABC) in Chapter 8, we proposed a two-stage FABC (TFABC) algorithm for multi-objective multiconstraints FJSSP. The three-value element encoding and decoding method is used to show the solution in TFABC algorithm. The MinEnd heuristic is employed to initialize population. Extensive computational experiments are carried out using eight instances from remanufacturing. The TFABC algorithm is compared to five heuristics. The results and comparisons demonstrate improved performance of TFABC algorithm for solving multi-objective multi-constraints FJSSP. 
The rest of this Chapter is organized as follows: Section 9.2 briefly describe the two objectives and two constraints of FJSSP. The proposed TFABC algorithm is presented in Section 9.3. Section 9.4 is for experimental results and comparisons. We conclude this Chapter in Section 9.5.

\subsection{Multi-objective and multi-constraint FJSSP}

In this Chapter, we address FJSSP with two constraints, fuzzy processing time and new job insertion. The fuzzy processing time constraint is modeled as a triangular fuzzy number (TFN) as follows.

$$
t_{i, j, k}=\left(t_{i, j, k}^{1}, t_{i, j, k}^{2}, t_{i, j, k}^{3}\right)
$$

where $t_{i, j, k}^{1}, t_{i, j, k}^{2}$ and $t_{i, j, k}^{3}$ are three probable processing time of operation $\boldsymbol{O}_{i, j}$ on machine $M_{k}$. The three operators for fuzzy number in Chapter 3 are employed to compare and rank the triangular fuzzy numbers. New job insertion constraint is solved by rescheduling. We employ the rescheduling strategy II proposed in Chapter 5 to obtain the rescheduling solution after new job insertion. The crucial idea of rescheduling strategy II is to reschedule the operations of new job(s) and the existing jobs' operations that are not yet started on the new job insertion time.

There is an example to show the effectiveness of rescheduling for new job(s) insertion constraint. Fig. 9 - 1 shows a Gantt chart of 5-jobs, 4-machines and 23-operations FJSSP with fuzzy processing time.

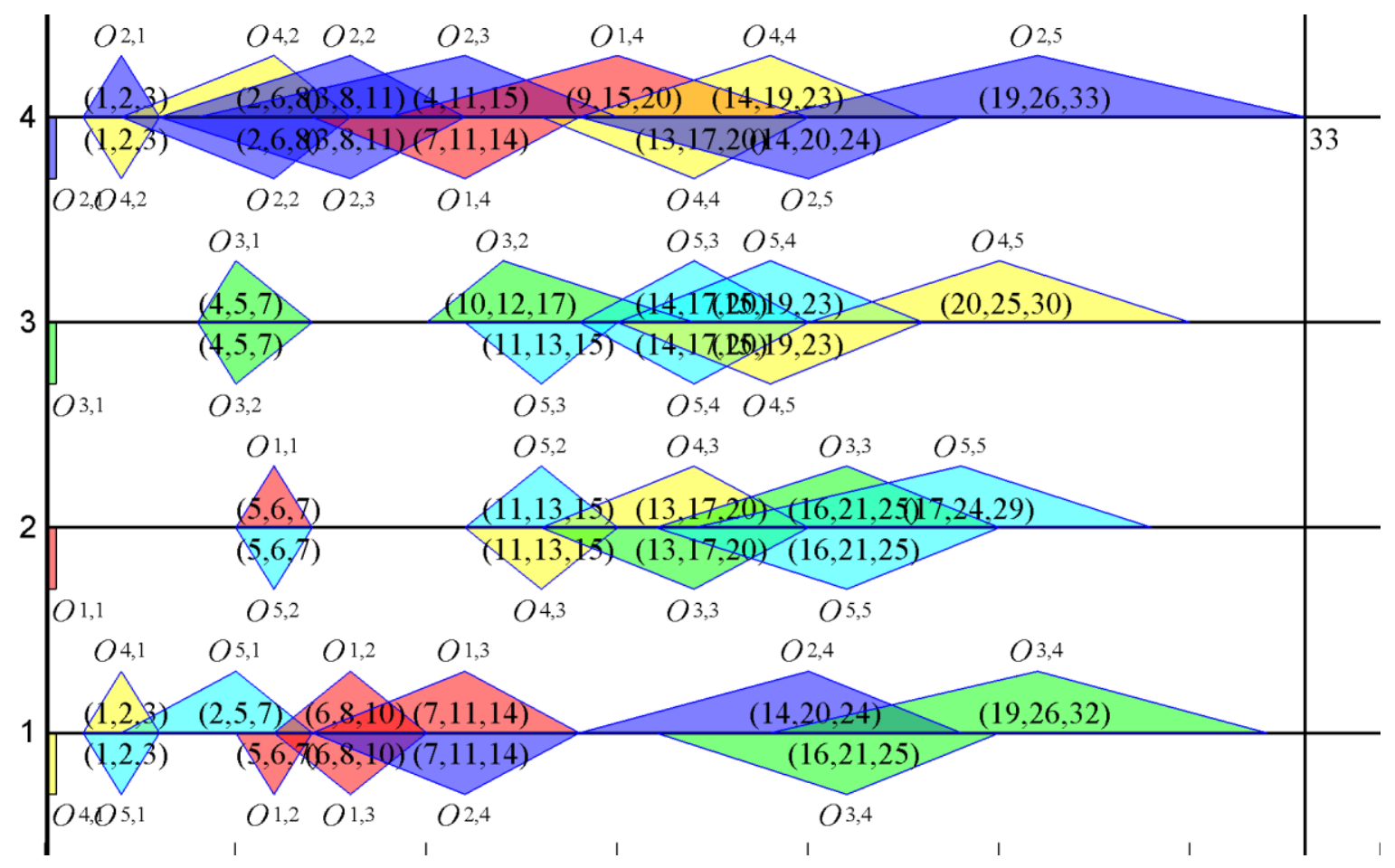

Fig. 9 - 1 An example Gantt chart for FJSSP with fuzzy processing time 


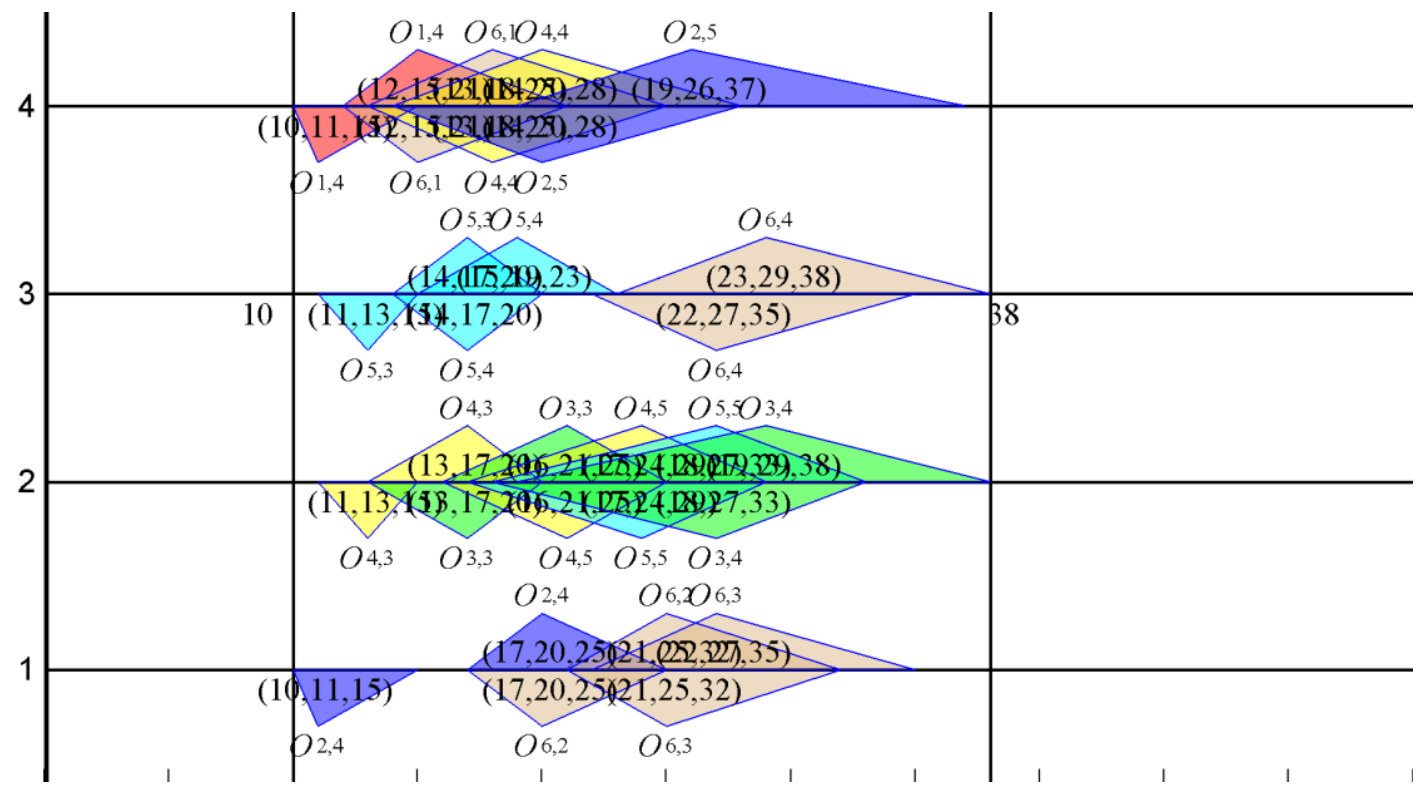

Fig. 9 - 2 The Gantt chart by rescheduling strategy II

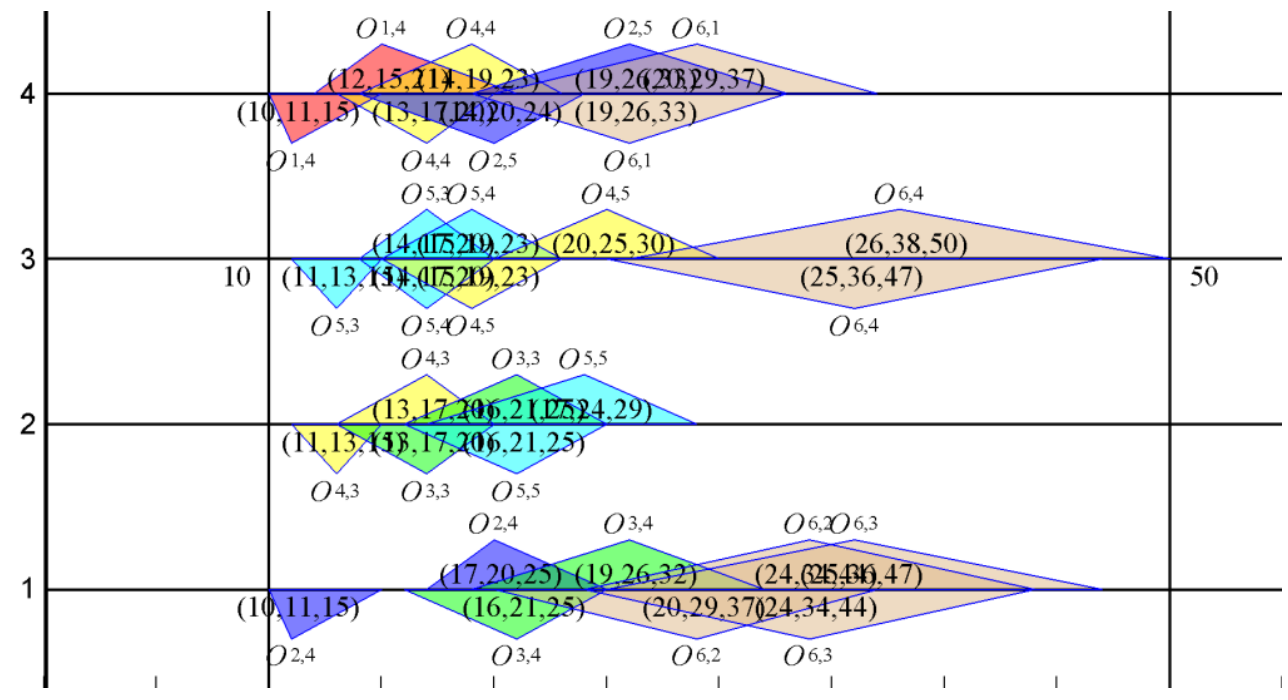

Fig. 9 - 3 The Gantt chart after rescheduling new job only

In this Gantt chart, the maximum fuzzy completion time is $(19,26,33)$. At time 10, a new job, job6, comes and is inserted into the existing solution. Rescheduling operator is activated to solve a new feasible solution for new job and existing jobs' operations that are not yet started at the new job insertion time. The Gantt chart after rescheduling is shown in Fig. 9 - 2. The rescheduling maximum fuzzy processing time is $(23,29,38)$. The result by rescheduling strategy II is compared to that by rescheduling strategy I in Chapter 5. The Gantt chart of rescheduling strategy I is shown in Fig. 9 - 3. It can be seen from Fig. 9 - 3 that the maximum fuzzy completion time of rescheduling strategy I is $(26,38,50)$. The rescheduling strategy II can improve the result of rescheduling strategy I by $(11.5 \%$, $23.6 \%, 24 \%)$. Hence, it is necessary to execute rescheduling and rescheduling strategy II is effective. 
Two objectives, maximum fuzzy completion time and maximum fuzzy machine workload, are considered. The fuzzy completion time of operation $O_{i, j}$ is a TFN as follows.

$$
C_{i, j}=\left(C_{i, j}^{1}, C_{i, j}^{2}, C_{i, j}^{3}\right)
$$

where $C_{i, j}^{1}, C_{i, j}^{2}$ and $C_{i, j}^{3}$ are three probable completion times of operation $\boldsymbol{O}_{i, j}$.

The fuzzy machine workload of machine $M_{k}$ is also a TFN as follows.

$$
w_{k}=\left(w_{k}^{1}, w_{k}^{2}, w_{k}^{3}\right)
$$

$w_{k}^{1}, w_{k}^{2}$ and $w_{k}^{3}$ are three probable machine workload of machine $M_{k}$.

The objective is to minimize maximum fuzzy completion time and maximum fuzzy machine workload. The maximum fuzzy completion time denoted by $C_{M}$ can be calculated by the following formula.

$$
\operatorname{Min} C_{M}=\max _{1 \leq i \leq n}\left\{C_{i}\right\}
$$

where $C_{i}$ is the fuzzy completion time of job $J_{i}$.

The maximum fuzzy machine workload denoted by $W_{M}$, can be calculated by:

$$
\operatorname{Min} W_{M}=\max _{1 \leq k \leq m}\left\{W_{k}\right\}
$$

where $W_{k}$ is the workload of machine $M_{k}$.

\subsection{Two-stage FABC algorithm for FJSSP}

The proposed TFABC algorithm includes two stages: scheduling stage and re-scheduling stage for new job insertion. The TFABC algorithm employed MinEnd heuristic, global minimizing processing time rule, and random rule to initialize the population. $90 \%$ solutions in the population are generated randomly, $5 \%$ solutions by MinEnd heuristic and $5 \%$ solutions by GS heuristic rule. In the procedure to generate new food source, changing processing machine operator and operation inserting operator are employed for exploitation search. PBetter or worse food source selection and scout bee operator are used for exploration search.

At the scheduling stage, the start times of all jobs and machines are the same. After the scheduling stage, the solution with the best result will be output, and all operations will be processed on the corresponding machines based on the best solution. When a new job comes and is inserted into existing schedule, the re-scheduling stage will be activated. All non-started operations of existing jobs and new job's operations will be rescheduled based on the re-scheduling strategy II. After rescheduling, new scheduling result replaces the existing one from the new job insertion time and is executed in the shop floor. 
The computational procedure of TFABC algorithm for scheduling and re-scheduling can be described as follows:

\section{Scheduling stage:}

Step1: Set parameters, employed bee number, onlooker bee number, scout bee number and so on.

Step2: Initialize population using MinEnd heuristic, GS rule and random rule.

Step3: Perform employed bee phase.

Step4: Perform onlooker bee phase.

Step5: Update the best solution.

Step6: If Limit is met, perform scout bee phase

Step7: If the stop criterion is not satisfied, go to Step 3; else, output the best solution.

\section{Re-scheduling stage:}

Step8: The new job comes and is inserted into the existing scheduling solution.

Step9: Calculate and record the available time of each job and each machine based on the new job insertion time.

Step10: For existing jobs, Re-calculate the number of operations that are not yet started. Add the new job(s) to the re-scheduling job set.

Step 11: Execute re-scheduling using the algorithm in scheduling stage.

Step12: Link up the re-scheduling results to executing scheduling results from the insertion time and execute the rescheduling solution in the shop floor.

For the TFABC algorithm, the computational complexity of the first stage is the same to the complexity of FABC algorithm in Chapter 8 . When the new job is inserted into the executing sequence, the first stage will be executed. Hence, the computational complexity of the TFABC algorithm is based on the number of times of new job insertion.

\subsection{Experiment evaluation and comparisons}

\subsubsection{Experiment setup}

Eight instances from remanufacturing industry is solved by the proposed TFABC algorithm. The instance size is from 5-jobs, 4-machines and 23-operations to 20-jobs, 15-machines and 355operations. 35 new jobs are inserted to existing scheduling sequence of eight instances when the instances are processing in the shop floor. Each insertion has different insertion time, job number and operation number. The detail information of eight instances with 35 new jobs is shown in Table 9 1. The first two columns are instance number and new job insertion times. The third column is the insertion time of new jobs. The last three columns are the corresponding job number, machine number 
and operation number. The first row of each instance shows the job number, machine number and operation number at scheduling stage.

Table 9 - 1 Information of eight instances and new jobs

\begin{tabular}{|c|c|c|c|c|c|}
\hline Instance & Insertion Times & Insertion time & Job Number & Machine Number & Operation number \\
\hline & 0 & 0 & 5 & \multirow{3}{*}{4} & 23 \\
\hline \multirow[t]{3}{*}{1} & 1 & 10 & 1 & & 4 \\
\hline & 2 & 15 & 1 & & 5 \\
\hline & 0 & 0 & 8 & & 64 \\
\hline \multirow[t]{3}{*}{2} & 1 & 12 & 1 & \multirow[t]{2}{*}{8} & 8 \\
\hline & 2 & 24 & 1 & & 8 \\
\hline & 0 & 0 & 10 & \multirow{4}{*}{6} & 81 \\
\hline \multirow{4}{*}{3} & 1 & 15 & 1 & & 8 \\
\hline & 2 & 25 & 1 & & 7 \\
\hline & 3 & 35 & 1 & & 6 \\
\hline & 0 & 0 & 10 & \multirow{3}{*}{10} & 100 \\
\hline \multirow{3}{*}{4} & 1 & 15 & 2 & & 20 \\
\hline & 2 & 30 & 2 & & 20 \\
\hline & 0 & 0 & 15 & \multirow{3}{*}{8} & 171 \\
\hline \multirow[t]{3}{*}{5} & 1 & 21 & 2 & & 18 \\
\hline & 2 & 47 & 2 & & 17 \\
\hline & 0 & 0 & 15 & \multirow{4}{*}{10} & 185 \\
\hline \multirow{4}{*}{6} & 1 & 14 & 2 & & 20 \\
\hline & 2 & 31 & 2 & & 19 \\
\hline & 3 & 44 & 2 & & 19 \\
\hline & 0 & 0 & 20 & \multirow{3}{*}{10} & 33 \\
\hline \multirow[t]{2}{*}{7} & 1 & 21 & 3 & & 38 \\
\hline & 2 & 52 & 3 & & 32 \\
\hline \multirow{4}{*}{8} & 0 & 0 & 20 & \multirow{4}{*}{15} & 355 \\
\hline & 1 & 18 & 3 & & 42 \\
\hline & 2 & 34 & 3 & & 42 \\
\hline & 3 & 49 & 2 & & 25 \\
\hline
\end{tabular}

The TFABC algorithm is coded in C++ and runs on Intel $2.40 \mathrm{GHz}$ PC with $2 \mathrm{~GB}$ memory. The parameters are set based on our previous research and the compared algorithms as follows: employed bee 50; onlooker bee 100 , scout bee 20 , Limit $50, \mathrm{P} 1=0.8, \mathrm{P} 2=0.95, \mathrm{P} 3=0.2$ and $\mathrm{P} 4=0.5$. For the previous four instances, the generation is set to 1000. For the last four instances, the generation is set to 4000 because the problem size of last four instances is larger than that of the previous instances. All experiments are carried out with 30 replications.

\subsubsection{Maximum fuzzy completion time objective}

To solve the eight instances with 19 times new job(s) insertion, TFABC algorithm is employed obtain the scheduling and rescheduling results. TFABC is compared to five heuristics, MinEnd, global minimizing processing time rule (GS), minimizing processing time (LS), most number of operations remaining $(\mathrm{MReO})$ and most work remaining rule $(\mathrm{MReW})$. The average maximum fuzzy completion time, best maximum fuzzy completion time and worst maximum fuzzy completion time by FABC 
algorithm and five heuristics are counted. The detail results for eight instances and 19 times new job(s) insertion are shown in Table 9 - 2 to Table 9 - 9.

Table 9 - 2 Maximum fuzzy processing time results of instance 1

\begin{tabular}{llllllllllll}
\hline Algorithm & $\begin{array}{l}\text { Insertion } \\
\text { time }\end{array}$ & \multicolumn{3}{c}{ Average } & \multicolumn{3}{c}{ Best } & \multicolumn{3}{c}{ Worst } & CPU \\
TFABC & 0 & 17.8 & 26.0 & 34.2 & 19 & 26 & 33 & 17 & 26 & 35 & 0.414 \\
MinEnd & & 24.6 & 38.4 & 51.1 & 20 & 31 & 42 & 35 & 48 & 63 & \\
GS & & 31.7 & 47.5 & 63.2 & 25 & 36 & 48 & 41 & 61 & 81 & \\
LS & & 29.0 & 46.6 & 61.2 & 17 & 34 & 45 & 40 & 61 & 79 & \\
MReO & & 28.9 & 41.8 & 55.9 & 16 & 30 & 42 & 57 & 76 & 96 & \\
MReW & & 29.6 & 47.3 & 64.7 & 20 & 33 & 45 & 58 & 80 & 107 & \\
& & & & & & & & & & & \\
TFABC & 1 & 22.8 & 29.0 & 38.0 & 21 & 29 & 38 & 23 & 29 & 38 & 0.266 \\
MinEnd & & 22.1 & 34.3 & 46.0 & 18 & 30 & 40 & 25 & 40 & 55 & \\
GS & & 26.7 & 42.7 & 57.6 & 22 & 34 & 47 & 39 & 55 & 75 & \\
LS & & 28.6 & 42.8 & 57.8 & 23 & 34 & 45 & 35 & 56 & 74 & \\
MReO & & 31.0 & 40.7 & 53.8 & 22 & 35 & 48 & 43 & 55 & 72 & \\
MReW & 29.8 & 45.0 & 60.2 & 20 & 34 & 46 & 57 & 77 & 102 & \\
& & & & & & & & & & \\
TFABC & 2 & 24.0 & 32.0 & 42.0 & 24 & 32 & 42 & 24 & 32 & 42 & 0.286 \\
MinEnd & & 27.9 & 37.3 & 51.5 & 26 & 32 & 46 & 32 & 43 & 56 & \\
GS & 32.2 & 47.1 & 64.2 & 26 & 36 & 51 & 48 & 70 & 97 & \\
LS & 30.0 & 46.7 & 63.9 & 25 & 37 & 52 & 38 & 57 & 78 & \\
MReO & & 34.8 & 46.5 & 60.5 & 28 & 39 & 53 & 47 & 65 & 86 & \\
MReW & 37.5 & 51.1 & 67.3 & 26 & 37 & 46 & 61 & 81 & 114 & \\
\hline
\end{tabular}

Table 9 - 2 shows the scheduling results and rescheduling results after two times new job insertion for instance 1 . The first and second columns are compared algorithms and new job(s) insertion times. The value " 0 " in the second column means the scheduling results for instance 1 while the values " 1 " and "2" mean the two times rescheduling results after two times new job(s) insertion. It can be seen from Table 9 - 2 that TFABC algorithm can obtain better results for average, best and worst measures than those by five compared heuristics in both scheduling stage and two times rescheduling stages. We can get the same conclusions for remaining seven instances from Table 9 - 3 to Table 9 - 9. For the remaining seven instances and 17 times new job(s) insertion, the TABC algorithm can obtain better scheduling and rescheduling results than those by five heuristics.

In addition, TFABC algorithm becomes more competitive than the five compared heuristics when the instance scale becomes larger from instance 1 to instance 8 . For example, TABC gets the average $(17.8,26.0,34.2)$, best $(19,26,33)$ and worst $(17,26,35)$ results for instance 1 . The best results of five heuristics are average $(24.6,38.4,51.1)$ by MinEnd, best $(16,30,42)$ by $\mathrm{MReO}$ and worst $(35$, 
$48,63)$ by MinEnd. The TFABC algorithm can improve the best results of five heuristics by average $(6.8,12.4,16.9)$, best $(-3,4,9)$ and worst $(18,22,28)$. For instance 8 , the TFABC algorithm can improve the best results of five heuristics by average $(32.6,52.5,71.7)$, best $(26,41,54)$ and worst $(30,68,96)$. After new job(s) insertion, the rescheduling results have the same conclusions. For example, the TFABC algorithm can improve the best instances 1 results of five heuristics by average $(3.9,5.3,9.5)$, best $(2,0,4)$ and worst $(8,11,14)$ after the second time new job insertion. For instance 8 , the TFABC algorithm can improve average, best and worst results by $(33.8,53.769 .8),(36,42,44)$ and $(50,78,91)$.

The CPU times of five heuristics for eight instances are very small and can be cancelled. For eight instances and 19 times new job(s) insertion, the TFABC algorithm's average CPU times in 30 repeat are shown in the last column of Table 9 - 2 to Table 9 - 9. The maximum CPU time is less than 2 minutes. Compared to the operation processing time in practical shop floor, the CPU times of TFABC algorithm can be cancelled.

For maximum fuzzy completion time objective, Fig. 9 - 4 to Fig. 9 - 6 show TFABC algorithm scheduling results for instance Reman 2 and two times new job insertions. Fig. 9 - 4 shows the scheduling results of instance Reman 2 and the maximum fuzzy completion time is $(17,29,58)$ on machine 6. Fig. 9 - 5 shows the rescheduling results after new job insertion at time 12. For all jobs and machines, the start time is at least 12. After rescheduling, the maximum completion time after new job 9 insertion is $(31,44,62)$ on machine 5 and machine 8. Fig. 9 - 6 shows the Gantt chart after the second time new job insertion on time 24 . The rescheduling result of maximum fuzzy processing time is $(38,51,75)$ on machine 6.

In summary, TFABC algorithm solves the eight instances from remanufacturing industry effectively. The results of TFABC are compared to those by five heuristics. The comparisons show that TFABC algorithm can obtain satisfactory results for real instances in remanufacturing industry and the TFABC algorithm needs very limited CPU time to get scheduling and rescheduling results.

Table 9 - 3 Maximum fuzzy processing time results of instance 2

\begin{tabular}{lllllllllllc}
\hline Algorithm & $\begin{array}{l}\text { Insertion } \\
\text { time }\end{array}$ & \multicolumn{3}{c}{ Average } & \multicolumn{3}{c}{ Best } & \multicolumn{3}{c}{ Worst } & $\begin{array}{c}\text { CPU } \\
\text { Time (s) }\end{array}$ \\
\hline TFABC & 0 & 24.3 & 40.2 & 56.7 & 17 & 39 & 58 & 25 & 43 & 60 & 1.483 \\
MinEnd & & 39.5 & 64.0 & 88.9 & 30 & 55 & 78 & 50 & 77 & 108 & \\
GS & & 53.6 & 83.8 & 116.3 & 44 & 66 & 91 & 73 & 116 & 160 & \\
LS & & 59.3 & 95.2 & 134.3 & 47 & 78 & 108 & 69 & 109 & 153 & \\
MReO & 49.9 & 75.5 & 104.8 & 37 & 59 & 80 & 77 & 100 & 140 & \\
MReW & 69.1 & 106.7 & 147.2 & 48 & 75 & 107 & 119 & 167 & 228 & \\
& & & & & & & & & & & \\
\hline
\end{tabular}




\begin{tabular}{lccccccccccc}
\hline TFABC & 1 & 31.9 & 45.0 & 59.2 & 31 & 44 & 62 & 30 & 45 & 61 & 0.895 \\
MinEnd & 45.8 & 68.5 & 92.4 & 41 & 54 & 77 & 55 & 83 & 114 & \\
GS & 60.6 & 88.9 & 122.5 & 48 & 73 & 100 & 70 & 109 & 147 & \\
LS & 61.4 & 94.1 & 130.6 & 59 & 79 & 112 & 75 & 118 & 164 & \\
MReO & 62.3 & 85.9 & 114.7 & 43 & 68 & 92 & 95 & 129 & 170 & \\
MReW & 73.3 & 109.4 & 150.3 & 53 & 83 & 114 & 114 & 164 & 213 & \\
& & & & & & & & & & \\
TFABC & 2 & 38.1 & 51.1 & 75.0 & 38 & 51 & 75 & 40 & 53 & 75 & 0.679 \\
MinEnd & 53.3 & 73.2 & 99.8 & 45 & 62 & 81 & 60 & 87 & 119 & \\
GS & 64.9 & 90.4 & 126.0 & 59 & 76 & 105 & 87 & 126 & 178 & \\
LS & 67.7 & 93.5 & 129.7 & 59 & 76 & 107 & 81 & 113 & 155 & \\
MReO & 65.3 & 87.5 & 119.0 & 47 & 67 & 100 & 91 & 121 & 164 & \\
MReW & 74.8 & 106.4 & 145.6 & 52 & 75 & 106 & 119 & 169 & 221 & \\
\hline
\end{tabular}

Table 9 - 4 Maximum fuzzy processing time results of instance 3

\begin{tabular}{|c|c|c|c|c|c|c|c|c|c|c|c|}
\hline \multirow{2}{*}{$\begin{array}{l}\text { Algorithm } \\
\text { TFABC }\end{array}$} & \multirow{2}{*}{$\begin{array}{l}\text { Insertion } \\
\text { time } \\
0\end{array}$} & \multicolumn{3}{|c|}{ Average } & \multicolumn{3}{|c|}{ Best } & \multicolumn{3}{|c|}{ Worst } & \multirow{2}{*}{$\begin{array}{c}\begin{array}{c}\text { CPU } \\
\text { Time (s) }\end{array} \\
1.971\end{array}$} \\
\hline & & 37.0 & 59.1 & 80.0 & 34 & 57 & 79 & 41 & 60 & 82 & \\
\hline MinEnd & & 62.4 & 98.7 & 133.8 & 49 & 84 & 113 & 74 & 119 & 166 & \\
\hline GS & & 76.7 & 117.7 & 159.5 & 56 & 91 & 120 & 91 & 133 & 179 & \\
\hline LS & & 82.9 & 128.7 & 173.9 & 67 & 101 & 135 & 108 & 160 & 210 & \\
\hline $\mathrm{MReO}$ & & 69.7 & 104.4 & 137.2 & 47 & 83 & 116 & 115 & 146 & 185 & \\
\hline MReW & & 94.9 & 144.6 & 194.0 & 61 & 102 & 138 & 142 & 198 & 262 & \\
\hline TFABC & 1 & 43.3 & 61.5 & 83.4 & 44 & 60 & 82 & 45 & 62 & 84 & 1.396 \\
\hline MinEnd & & 66.6 & 99.2 & 132.9 & 62 & 78 & 101 & 74 & 116 & 154 & \\
\hline GS & & 81.8 & 119.4 & 160.2 & 65 & 103 & 144 & 93 & 138 & 189 & \\
\hline LS & & 89.0 & 134.0 & 181.1 & 72 & 104 & 138 & 116 & 176 & 237 & \\
\hline $\mathrm{MReO}$ & & 68.5 & 100.5 & 133.6 & 52 & 85 & 115 & 101 & 138 & 177 & \\
\hline MReW & & 98.4 & 147.1 & 196.3 & 65 & 98 & 134 & 171 & 235 & 310 & \\
\hline TFABC & 2 & 52.9 & 68.7 & 91.7 & 54 & 68 & 90 & 56 & 71 & 91 & 1.101 \\
\hline MinEnd & & 75.2 & 103.8 & 138.4 & 66 & 90 & 121 & 85 & 117 & 153 & \\
\hline GS & & 95.4 & 131.9 & 176.5 & 81 & 106 & 147 & 115 & 167 & 225 & \\
\hline LS & & 99.2 & 140.8 & 187.9 & 83 & 119 & 158 & 117 & 169 & 224 & \\
\hline $\mathrm{MReO}$ & & 85.4 & 114.9 & 150.4 & 75 & 94 & 122 & 113 & 148 & 194 & \\
\hline MReW & & 112.3 & 154.3 & 203.3 & 87 & 119 & 155 & 164 & 222 & 291 & \\
\hline TFABC & 3 & 58.8 & 71.5 & 98.9 & 59 & 71 & 99 & 60 & 73 & 99 & 0.833 \\
\hline MinEnd & & 82.0 & 106.6 & 140.9 & 74 & 97 & 124 & 95 & 129 & 168 & \\
\hline GS & & 97.9 & 132.4 & 179.1 & 78 & 106 & 140 & 114 & 157 & 208 & \\
\hline LS & & 105.8 & 143.9 & 189.1 & 94 & 126 & 164 & 130 & 177 & 239 & \\
\hline $\mathrm{MReO}$ & & 84.9 & 110.4 & 145.7 & 76 & 93 & 124 & 117 & 148 & 195 & \\
\hline MReW & & 111.8 & 151.1 & 200.2 & 84 & 120 & 166 & 161 & 216 & 290 & \\
\hline
\end{tabular}


Table 9 - 5 Maximum fuzzy processing time results of instance 4

\begin{tabular}{llllllllllll}
\hline Algorithm & $\begin{array}{l}\text { Insertion } \\
\text { time }\end{array}$ & \multicolumn{3}{c}{ Average } & \multicolumn{3}{c}{ Best } & & Worst & $\begin{array}{c}\text { CPU } \\
\text { Time (s) }\end{array}$ \\
\hline TFABC & 0 & 33.8 & 50.8 & 70.6 & 28 & 49 & 73 & 54 & 69 & 92 & 2.768 \\
MinEnd & & 49.8 & 78.1 & 109.1 & 40 & 66 & 97 & 62 & 91 & 126 & \\
GS & & 67.2 & 103.9 & 141.7 & 58 & 91 & 126 & 94 & 131 & 176 & \\
LS & & 74.0 & 118.9 & 166.4 & 55 & 101 & 144 & 91 & 142 & 198 & \\
MReO & 64.4 & 95.0 & 129.6 & 48 & 77 & 106 & 112 & 147 & 188 & \\
MReW & 91.0 & 140.0 & 192.6 & 56 & 102 & 143 & 146 & 199 & 275 & \\
& & & & & & & & & & & \\
TFABC & 1 & 39.4 & 56.8 & 76.9 & 38 & 55 & 74 & 37 & 59 & 84 & 1.948 \\
MinEnd & & 60.8 & 83.9 & 114.3 & 50 & 71 & 105 & 74 & 101 & 136 & \\
GS & & 78.1 & 113.1 & 154.8 & 70 & 91 & 123 & 94 & 136 & 182 & \\
LS & 75.7 & 119.5 & 167.6 & 67 & 104 & 145 & 91 & 140 & 193 & \\
MReO & 80.6 & 115.3 & 156.5 & 61 & 86 & 113 & 108 & 147 & 200 & \\
MReW & 97.8 & 142.1 & 194.0 & 58 & 100 & 142 & 144 & 193 & 254 & \\
& & & & & & & & & & \\
TFABC & 2 & 53.0 & 68.7 & 92.0 & 51 & 68 & 93 & 38 & 54 & 73 & 1.565 \\
MinEnd & & 66.5 & 90.4 & 123.3 & 56 & 78 & 107 & 81 & 115 & 154 & \\
GS & 90.0 & 121.1 & 162.1 & 73 & 95 & 130 & 105 & 141 & 173 & \\
LS & 88.6 & 132.5 & 181.2 & 78 & 110 & 150 & 104 & 154 & 208 & \\
MReO & 93.0 & 124.3 & 165.5 & 70 & 96 & 132 & 116 & 158 & 210 & \\
MReW & 112.1 & 158.4 & 215.1 & 79 & 121 & 166 & 173 & 227 & 296 & \\
\hline
\end{tabular}

Table 9 - 6 Maximum fuzzy processing time results of instance 5

\begin{tabular}{llcccccccccc}
\hline Algorithm & $\begin{array}{l}\text { Insertion } \\
\text { time }\end{array}$ & \multicolumn{3}{c}{ Average } & \multicolumn{3}{c}{ Best } & & Worst & $\begin{array}{c}\text { CPU } \\
\text { Time (s) }\end{array}$ \\
\hline TFABC & 0 & 51.8 & 83.4 & 115.0 & 51 & 81 & 114 & 56 & 87 & 115 & 23.617 \\
MinEnd & & 83.2 & 132.2 & 181.3 & 68 & 119 & 169 & 105 & 165 & 227 & \\
GS & & 110.6 & 172.4 & 234.8 & 100 & 151 & 205 & 138 & 209 & 273 & \\
LS & & 143.5 & 217.5 & 291.4 & 120 & 182 & 242 & 166 & 248 & 334 & \\
MReO & 106.2 & 165.2 & 223.9 & 88 & 139 & 194 & 166 & 233 & 314 & \\
MReW & 147.6 & 234.8 & 317.4 & 111 & 173 & 230 & 237 & 324 & 418 & \\
& & & & & & & & & & & \\
TFABC & 1 & 63.1 & 90.4 & 125.6 & 60 & 92 & 120 & 66 & 91 & 128 & 16.216 \\
MinEnd & & 98.7 & 142.5 & 194.4 & 89 & 128 & 174 & 113 & 160 & 219 & \\
GS & 117.9 & 172.7 & 234.4 & 94 & 144 & 205 & 149 & 205 & 286 & \\
LS & 133.2 & 203.2 & 276.3 & 112 & 165 & 225 & 153 & 234 & 320 & \\
MReO & 125.0 & 181.9 & 247.2 & 93 & 147 & 206 & 186 & 246 & 332 & \\
MReW & 154.5 & 231.8 & 314.5 & 118 & 186 & 261 & 253 & 341 & 450 & \\
& & & & & & & & & & & \\
TFABC & 2 & 75.8 & 98.0 & 132.8 & 77 & 97 & 128 & 79 & 99 & 135 & 9.743 \\
MinEnd & 109.8 & 150.4 & 202.8 & 100 & 132 & 179 & 119 & 164 & 223 & \\
GS & 123.0 & 168.9 & 229.3 & 107 & 143 & 200 & 142 & 197 & 267 & \\
LS & 150.4 & 210.3 & 282.5 & 132 & 188 & 250 & 172 & 248 & 325 & \\
\hline
\end{tabular}




\begin{tabular}{llllllllll}
\hline MReO & 140.4 & 187.7 & 248.5 & 112 & 155 & 209 & 190 & 240 & 321 \\
$\mathrm{MReW}$ & 177.5 & 243.5 & 329.7 & 130 & 195 & 270 & 250 & 334 & 442 \\
\hline
\end{tabular}

Table 9 - 7 Maximum fuzzy processing time results of instance 6

\begin{tabular}{|c|c|c|c|c|c|c|c|c|c|c|c|}
\hline Algorithm & $\begin{array}{l}\text { Insertion } \\
\text { time }\end{array}$ & & Average & & & Best & & & Worst & & $\begin{array}{c}\text { CPU } \\
\text { Time (s) }\end{array}$ \\
\hline TFABC & 0 & 44.2 & 72.1 & 98.5 & 38 & 70 & 100 & 45 & 74 & 103 & 28.123 \\
\hline MinEnd & & 68.6 & 113.1 & 154.7 & 57 & 102 & 144 & 85 & 142 & 193 & \\
\hline GS & & 96.0 & 156.1 & 213.9 & 84 & 133 & 173 & 108 & 178 & 246 & \\
\hline $\mathrm{LS}$ & & 109.6 & 178.0 & 243.3 & 83 & 154 & 215 & 136 & 214 & 293 & \\
\hline $\mathrm{MReO}$ & & 102.8 & 153.5 & 204.8 & 66 & 114 & 153 & 162 & 210 & 271 & \\
\hline MReW & & 142.2 & 223.1 & 302.8 & 94 & 162 & 219 & 219 & 313 & 415 & \\
\hline TFABC & 1 & 49.6 & 76.6 & 104.5 & 52 & 74 & 101 & 48 & 78 & 109 & 21.796 \\
\hline MinEnd & & 77.3 & 121.9 & 167.0 & 72 & 110 & 152 & 82 & 137 & 189 & \\
\hline GS & & 97.2 & 156.7 & 214.0 & 84 & 129 & 176 & 105 & 176 & 243 & \\
\hline LS & & 113.1 & 180.1 & 246.0 & 99 & 162 & 220 & 139 & 212 & 288 & \\
\hline $\mathrm{MReO}$ & & 103.1 & 154.3 & 210.0 & 79 & 122 & 169 & 158 & 218 & 281 & \\
\hline MReW & & 164.0 & 247.4 & 331.5 & 109 & 174 & 238 & 261 & 364 & 472 & \\
\hline TFABC & 2 & 58.4 & 80.9 & 112.6 & 54 & 81 & 112 & 60 & 81 & 116 & 15.813 \\
\hline MinEnd & & 90.0 & 129.3 & 177.8 & 88 & 115 & 161 & 102 & 142 & 192 & \\
\hline GS & & 100.5 & 154.2 & 213.9 & 89 & 132 & 184 & 109 & 178 & 244 & \\
\hline LS & & 117.0 & 184.5 & 252.1 & 100 & 163 & 223 & 144 & 220 & 303 & \\
\hline $\mathrm{MReO}$ & & 110.9 & 157.9 & 209.6 & 83 & 120 & 165 & 159 & 211 & 264 & \\
\hline MReW & & 164.2 & 243.4 & 326.3 & 109 & 174 & 245 & 240 & 325 & 427 & \\
\hline TFABC & 3 & 107.7 & 160.1 & 217.0 & 65 & 85 & 115 & 64 & 86 & 120 & 12.475 \\
\hline MinEnd & & 91.1 & 127.6 & 173.6 & 88 & 120 & 158 & 96 & 136 & 183 & \\
\hline GS & & 108.7 & 157.7 & 216.9 & 101 & 138 & 185 & 121 & 177 & 238 & \\
\hline LS & & 118.1 & 185.5 & 254.4 & 92 & 152 & 209 & 140 & 215 & 296 & \\
\hline $\mathrm{MReO}$ & & 115.4 & 159.6 & 216.1 & 95 & 136 & 180 & 169 & 226 & 302 & \\
\hline MReW & & 153.3 & 225.8 & 307.7 & 124 & 186 & 259 & 234 & 317 & 412 & \\
\hline
\end{tabular}

Table 9 - 8 Maximum fuzzy processing time results of instance 7

\begin{tabular}{llllllllllll}
\hline Algorithm & $\begin{array}{l}\text { Insertion } \\
\text { time }\end{array}$ & \multicolumn{3}{c}{ Average } & \multicolumn{3}{c}{ Best } & & Worst & $\begin{array}{c}\text { CPU } \\
\text { Time (s) }\end{array}$ \\
\hline TFABC & 0 & 59.2 & 107.5 & 153.4 & 53 & 104 & 155 & 64 & 111 & 155 & 80.307 \\
MinEnd & & 98.4 & 169.6 & 236.2 & 88 & 157 & 209 & 111 & 182 & 255 & \\
GS & & 132.9 & 228.4 & 315.7 & 112 & 205 & 286 & 153 & 255 & 354 & \\
LS & & 149.8 & 265.4 & 368.5 & 136 & 236 & 330 & 169 & 297 & 414 \\
MReO & 159.1 & 248.1 & 336.0 & 100 & 177 & 248 & 231 & 342 & 446 \\
MReW & & 189.8 & 322.5 & 446.9 & 134 & 258 & 366 & 329 & 471 & 631 \\
& & & & & & & & & & & \\
TFABC & 1 & 74.6 & 121.3 & 171.2 & 72 & 118 & 170 & 81 & 123 & 174 & 61.628 \\
\hline
\end{tabular}




\begin{tabular}{|c|c|c|c|c|c|c|c|c|c|c|c|}
\hline MinEnd & & 114.1 & 183.1 & 252.2 & 96 & 168 & 229 & 129 & 203 & 284 & \\
\hline GS & & 144.5 & 237.0 & 327.2 & 126 & 208 & 292 & 162 & 274 & 374 & \\
\hline LS & & 169.6 & 279.0 & 386.6 & 141 & 244 & 347 & 206 & 324 & 445 & \\
\hline $\mathrm{MReO}$ & & 156.4 & 237.0 & 323.4 & 117 & 175 & 245 & 252 & 347 & 459 & \\
\hline MReW & & 216.0 & 343.7 & 469.5 & 144 & 273 & 394 & 336 & 491 & 645 & \\
\hline TFABC & 2 & 95.6 & 130.5 & 183.6 & 96 & 130 & 179 & 94 & 135 & 188 & 34.865 \\
\hline MinEnd & & 133.9 & 194.4 & 265.9 & 123 & 184 & 252 & 151 & 208 & 282 & \\
\hline GS & & 167.9 & 255.4 & 350.3 & 152 & 236 & 316 & 181 & 279 & 376 & \\
\hline LS & & 190.6 & 296.3 & 410.3 & 177 & 265 & 378 & 226 & 330 & 452 & \\
\hline $\mathrm{MReO}$ & & 164.0 & 235.2 & 325.3 & 126 & 191 & 270 & 243 & 328 & 443 & \\
\hline MReW & & 222.5 & 334.8 & 464.4 & 164 & 260 & 367 & 332 & 443 & 615 & \\
\hline
\end{tabular}

Table 9 - 9 Maximum fuzzy processing time results of instance 8

\begin{tabular}{|c|c|c|c|c|c|c|c|c|c|c|c|}
\hline \multirow{2}{*}{$\begin{array}{l}\text { Algorithm } \\
\text { TFABC }\end{array}$} & \multirow{2}{*}{$\begin{array}{l}\text { Insertion } \\
\text { time } \\
0\end{array}$} & \multicolumn{3}{|c|}{ Average } & \multicolumn{3}{|c|}{ Best } & \multicolumn{3}{|c|}{ Worst } & \multirow{2}{*}{$\begin{array}{c}\text { CPU } \\
\text { Time (s) } \\
113.064\end{array}$} \\
\hline & & 56.8 & 92.7 & 127.1 & 53 & 89 & 124 & 58 & 95 & 134 & \\
\hline MinEnd & & 89.4 & 145.1 & 198.8 & 79 & 130 & 178 & 88 & 163 & 230 & \\
\hline GS & & 120.2 & 202.2 & 278.0 & 102 & 178 & 251 & 139 & 231 & 317 & \\
\hline $\mathrm{LS}$ & & 143.5 & 239.1 & 329.8 & 131 & 207 & 282 & 173 & 284 & 390 & \\
\hline $\mathrm{MReO}$ & & 130.2 & 204.9 & 279.3 & 103 & 172 & 231 & 199 & 274 & 367 & \\
\hline MReW & & 228.9 & 358.1 & 485.7 & 152 & 274 & 374 & 326 & 443 & 581 & \\
\hline TFABC & 1 & 64.4 & 100.5 & 141.6 & 64 & 93 & 133 & 71 & 100 & 134 & 81.105 \\
\hline MinEnd & & 97.3 & 152.0 & 207.4 & 91 & 136 & 189 & 101 & 164 & 228 & \\
\hline GS & & 130.9 & 211.4 & 290.3 & 111 & 191 & 266 & 146 & 243 & 341 & \\
\hline LS & & 145.0 & 242.6 & 335.6 & 114 & 201 & 278 & 169 & 289 & 395 & \\
\hline $\mathrm{MReO}$ & & 142.6 & 217.3 & 294.5 & 102 & 170 & 231 & 221 & 292 & 396 & \\
\hline MReW & & 212.0 & 336.4 & 457.9 & 159 & 287 & 398 & 341 & 461 & 591 & \\
\hline TFABC & 2 & 76.2 & 107.2 & 147.9 & 75 & 101 & 141 & 74 & 108 & 145 & 60.931 \\
\hline MinEnd & & 108.9 & 158.9 & 214.5 & 97 & 147 & 199 & 124 & 174 & 227 & \\
\hline GS & & 138.9 & 217.8 & 300.6 & 112 & 189 & 269 & 155 & 254 & 354 & \\
\hline LS & & 155.0 & 242.4 & 336.9 & 134 & 208 & 291 & 182 & 280 & 388 & \\
\hline $\mathrm{MReO}$ & & 148.3 & 218.1 & 294.1 & 111 & 174 & 242 & 233 & 314 & 414 & \\
\hline MReW & & 222.3 & 340.6 & 462.0 & 152 & 260 & 364 & 346 & 477 & 624 & \\
\hline TFABC & 3 & 81.8 & 107.2 & 149.6 & 76 & 105 & 154 & 82 & 108 & 158 & 38.420 \\
\hline MinEnd & & 115.6 & 161.0 & 219.4 & 112 & 147 & 198 & 132 & 186 & 249 & \\
\hline GS & & 145.4 & 215.3 & 298.1 & 134 & 190 & 261 & 171 & 243 & 331 & \\
\hline LS & & 158.1 & 234.6 & 325.6 & 142 & 206 & 278 & 185 & 268 & 369 & \\
\hline $\mathrm{MReO}$ & & 158.1 & 222.5 & 300.3 & 126 & 186 & 244 & 229 & 298 & 401 & \\
\hline MReW & & 206.7 & 319.2 & 439.1 & 180 & 272 & 382 & 337 & 468 & 603 & \\
\hline
\end{tabular}




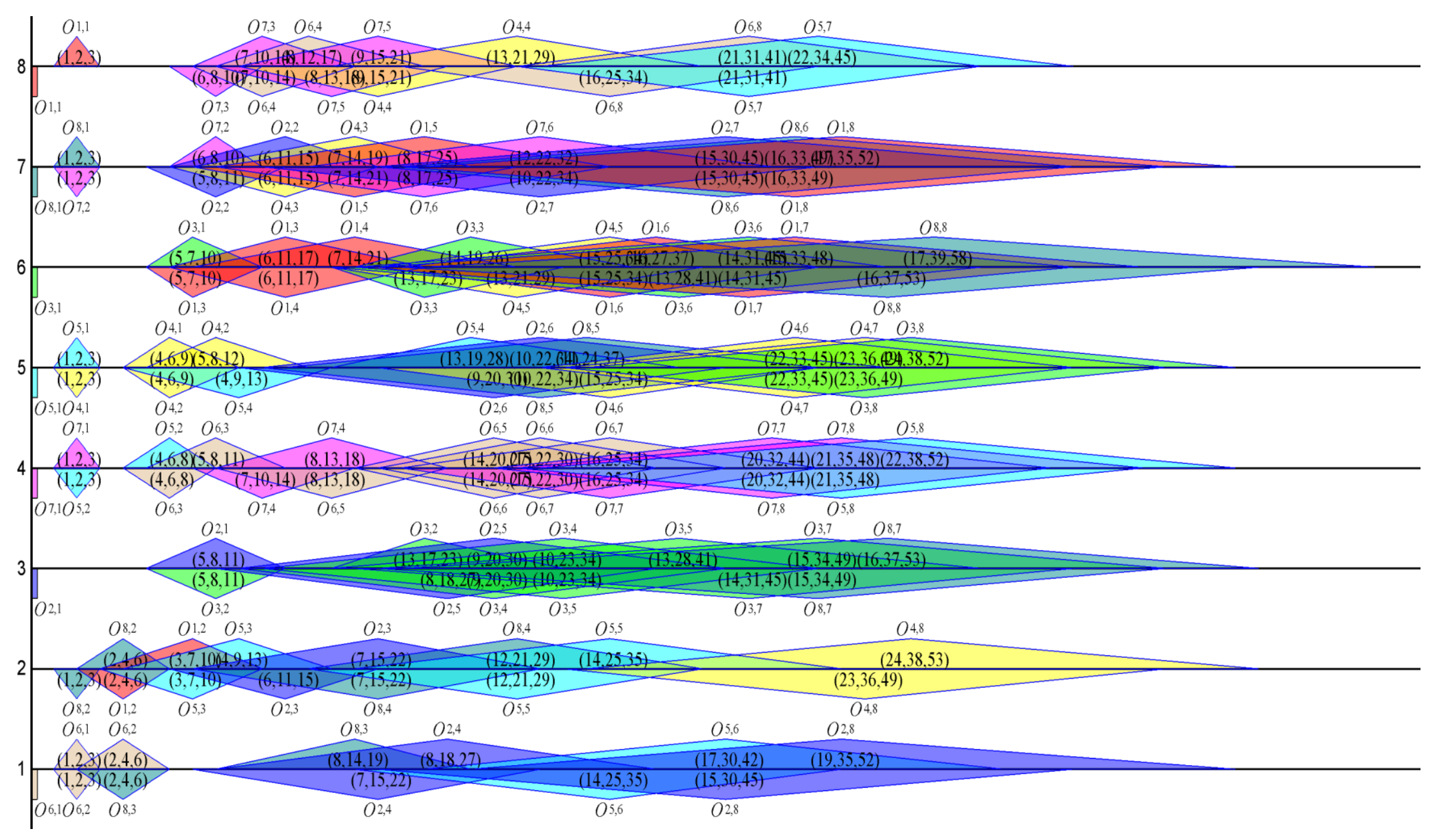

Fig. 9 - 4 Gantt chart of instance Reman 2 with minimum maximum fuzzy completion time 


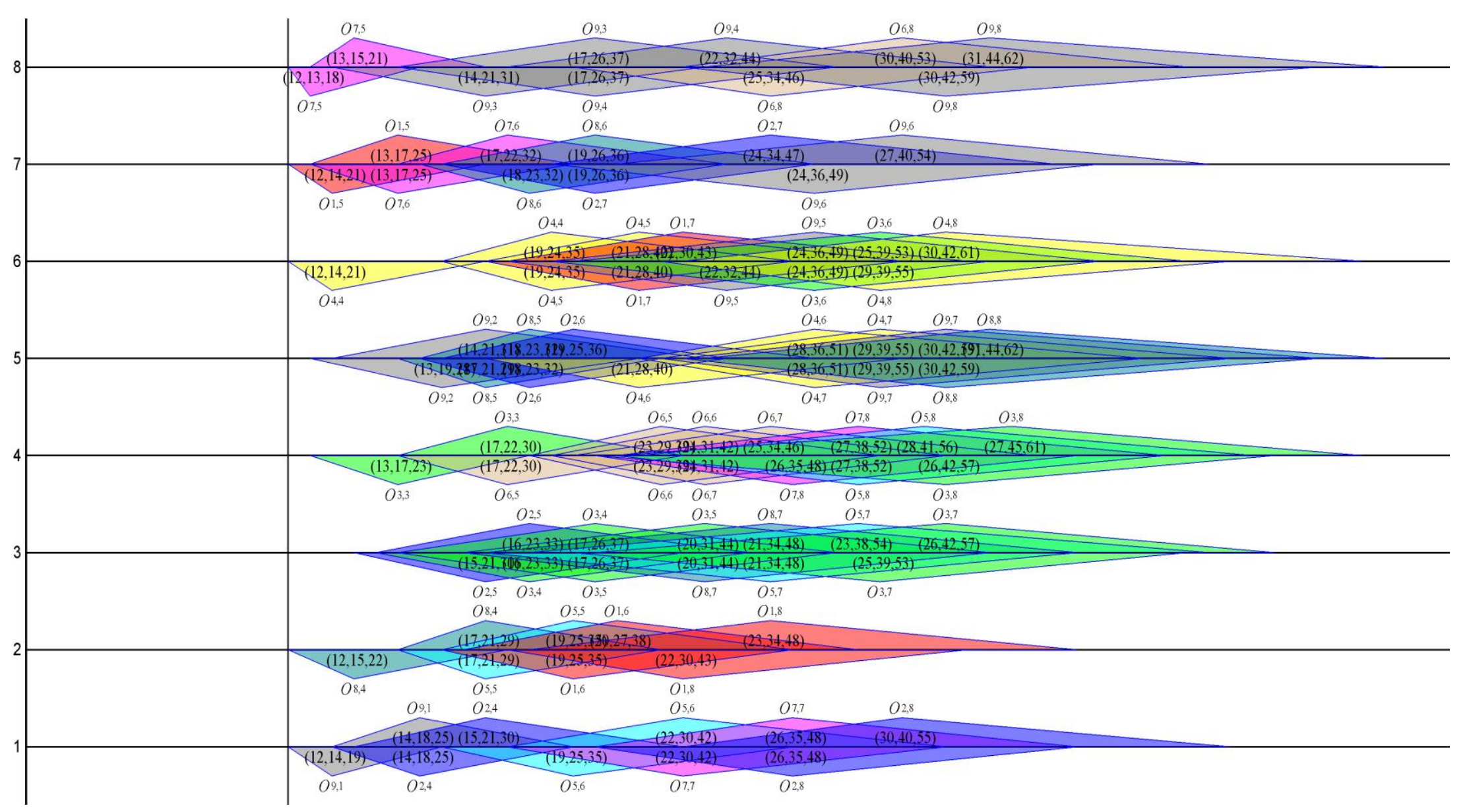

Fig. 9 - 5 First rescheduling Gantt chart with best maximum fuzzy completion time 


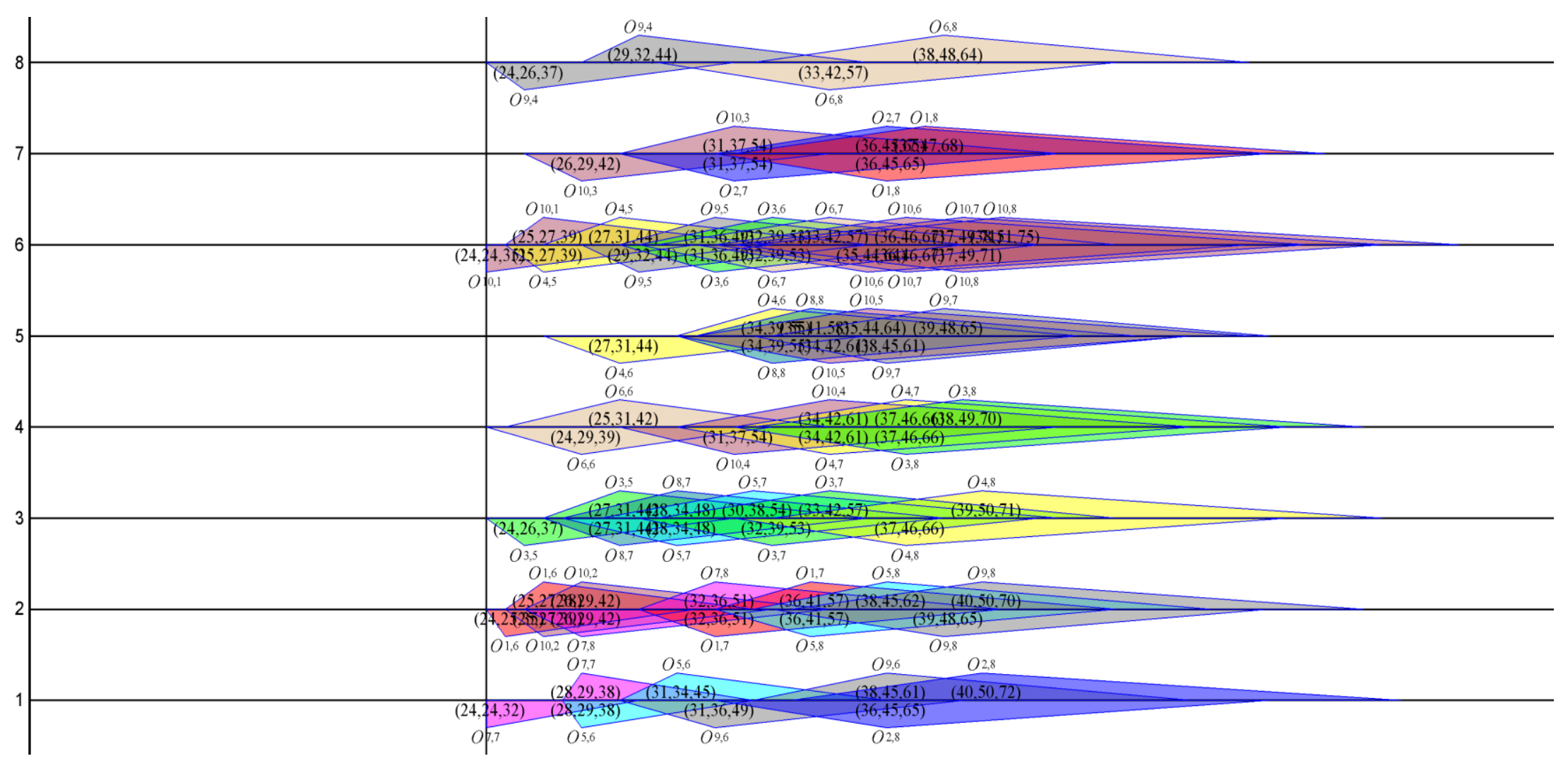

Fig. 9 - 6 Second rescheduling Gantt chart with best maximum fuzzy completion time 


\subsubsection{Maximum fuzzy machine workload objective}

In both scheduling and rescheduling stages, we calculate the maximum machine workload for current scheduling stage. For maximum fuzzy machine workload objective, we compare FABC algorithm to five heuristics, MinEnd, GS, LS, MReW and MaxReO. To show FABC algorithm performance, the following formula is employed:

$$
\operatorname{Imp}(\mathrm{i})=\frac{\left(\mathrm{t}_{1}^{\mathrm{i}}+2 \mathrm{t}_{2}^{\mathrm{i}}+\mathrm{t}_{3}^{\mathrm{i}}\right) / 4-\left(\mathrm{t}_{1}^{0}+2 \mathrm{t}_{2}^{0}+\mathrm{t}_{3}^{0}\right) / 4}{\left(\mathrm{t}_{1}^{0}+2 \mathrm{t}_{2}^{0}+\mathrm{t}_{3}^{0}\right) / 4} \times 100 \%
$$

where $t_{1}^{\mathrm{i}}, \mathrm{t}_{2}^{\mathrm{i}}$ and $\mathrm{t}_{3}^{\mathrm{i}}$ are the results of $\mathrm{i}^{\text {th }}$ heuristic represented by TFN; $t_{1}^{0}, \mathrm{t}_{2}^{0}$ and $\mathrm{t}_{3}^{0}$ are the result of TFABC algorithm. $\left(t_{1}^{\mathrm{i}}+2 \mathrm{t}_{2}^{\mathrm{i}}+\mathrm{t}_{3}^{\mathrm{i}}\right) / 4$ is the first criterion to compare two TFNs. Because the results by TFABC algorithm are better than those by six heuristics, the first criterion is enough to compare them. It is clear that the larger the $\operatorname{Imp}(\mathrm{i})$ is, more competitive TFABC algorithm than $\mathrm{i}^{\text {th }}$ heuristic. For eight instances, the maximum fuzzy machine workload results of TFABC and five heuristics are evaluated. The $\operatorname{Imp}(i)$ results of five heuristics to TFABC algorithm are also shown in statistics. All the results are shown in Table 9 - 10 to Table 9 -17. Each table shows the maximum machine workload of one instance in scheduling and rescheduling stages.

Table 9 - 10 shows the maximum machine workload results for instance 1and two times new job insertion. The first and second columns are compared algorithms and new job(s) insertion times. The value " 0 " in the second column means the scheduling results for instance 1 while the values " 1 " and " 2 " mean the two times rescheduling results after two times new job(s) insertion. It can be seen from Table 9 - 10 that TFABC algorithm can obtain the better results for average and worst measures than those by five compared heuristics in both scheduling stage and two times rescheduling stages. For the best measure, TFABC can just find competitive results in scheduling stage. The MReW heuristic get the most competitive results in the first rescheduling stage while MinEnd, GS and MReO heuristics obtain the most competitive results in the second rescheduling stage.

It can be seen from Table 9 - 11 to Table 9 - 17 that the TFABC algorithm can obtain the most competitive results for average, best and worst measures in scheduling and rescheduling stages. For instance 2, the average values of five heuristics are larger than those of FABC by at least $29.8 \%, 6.4 \%$ and $23.2 \%$ in scheduling and two times rescheduling stages. The corresponding values of best and worst measures are by at least $20.2 \%, 5.7 \%$ and $13.4 \%$ and at least $45.4 \%, 35.5 \%$ and $37.1 \%$ respectively. For instance 3 to instance 8 and 15 times new job insertion, We can get the similar comparison conclusion from Table 9 - 12 to Table 9 - 17.

The CPU times of five heuristics for eight instances and new job insertion are very small and can be cancelled. For eight instances and 19 times new job(s) insertion, the TFABC algorithm's average CPU 
times in 30 repeat are shown in Table 9 - 18. The maximum CPU time is less than 2 minutes. Compared to the operation processing time in practical shop floor, the CPU times of TFABC algorithm are very limited.

For maximum fuzzy machine workload objective, Fig. 9 - 7 to Fig. 9 - 9 show TFABC algorithm scheduling results for instance Reman2 and two times new job insertions. Fig. 9 - 7 shows the scheduling results of instance Reman 2 and the maximum fuzzy machine workload is $(13,30,41)$ on machine 4. Fig. $9-8$ shows the rescheduling results after new job insertion at time 12. After rescheduling, the maximum machine workload after new job 9 insertion is $(17,26,37)$ on machine 1. Fig. 9 - 9 shows the Gantt chart after the second time new job insertion on time 24. The rescheduling result of maximum fuzzy machine workload is $(15,24,34)$ on machine 7.

In summary, TFABC can obtain most competitive maximum machine workload results for all instances except the two times new job insertion in instance 1. The comparisons show that TFABC algorithm can obtain satisfactory results for real instances in remanufacturing industry and the TFABC algorithm needs very limited CPU time to get scheduling and rescheduling maximum machine workload results.

Table 9 - 10 Maximum fuzzy machine workload results of instance 1

\begin{tabular}{llrlrrrrrrrrrr}
\hline Algorithm & $\begin{array}{l}\text { Insertion } \\
\text { times }\end{array}$ & \multicolumn{2}{c}{ Average } & $\begin{array}{l}\text { Imp } \\
(\%)\end{array}$ & & Best & $\begin{array}{r}\text { Imp } \\
(\%)\end{array}$ & Worst & $\begin{array}{r}\text { Imp } \\
(\%)\end{array}$ \\
\hline TFABC & 0 & 13.0 & 22.0 & 30.0 & & 13 & 22 & 30 & & 13 & 22 & 30 & \\
MinEnd & & 17.9 & 28.7 & 38.6 & 31.0 & 14 & 24 & 33 & 9.2 & 21 & 35 & 47 & 58.6 \\
GS & & 17.0 & 27.4 & 36.7 & 24.6 & 14 & 24 & 33 & 9.2 & 21 & 31 & 41 & 42.5 \\
LS & & 15.0 & 28.0 & 39.0 & 26.4 & 15 & 28 & 39 & 26.4 & 15 & 28 & 39 & 26.4 \\
MReO & 22.5 & 35.1 & 47.8 & 61.5 & 14 & 24 & 34 & 10.3 & 53 & 71 & 90 & 227.6 \\
MReW & 21.2 & 33.8 & 46.2 & 55.2 & 17 & 26 & 35 & 19.5 & 41 & 56 & 75 & 162.1 \\
& & & & & & & & & & & & & 0.0 \\
TFABC & 1 & 10.0 & 18.0 & 26.0 & & 10 & 18 & 26 & & 10 & 18 & 26 & 0.0 \\
MinEnd & 15.2 & 23.0 & 30.9 & 28.1 & 12 & 18 & 25 & 1.4 & 24 & 33 & 43 & 84.7 \\
GS & 13.0 & 20.3 & 26.7 & 11.6 & 13 & 19 & 24 & 4.2 & 16 & 24 & 31 & 31.9 \\
LS & 16.0 & 31.0 & 43.0 & 68.1 & 16 & 31 & 43 & 68.1 & 16 & 31 & 43 & 68.1 \\
MReO & 15.5 & 24.3 & 32.7 & 34.4 & 10 & 19 & 27 & 4.2 & 30 & 39 & 53 & 123.6 \\
MReW & 18.3 & 28.8 & 39.5 & 60.3 & 10 & 16 & 23 & -9.7 & 42 & 56 & 71 & 212.5 \\
& & & & & & & & & & & & & 0.0 \\
TFABC & 2 & 9.0 & 19.0 & 27.0 & & 9 & 19 & 27 & & 9 & 19 & 27 & 0.0 \\
MinEnd & 12.7 & 20.5 & 28.2 & 10.6 & 10 & 17 & 25 & -6.8 & 21 & 27 & 35 & 48.6 \\
GS & 12.8 & 19.4 & 26.4 & 5.4 & 10 & 17 & 25 & -6.8 & 19 & 24 & 30 & 31.1 \\
LS & 12.0 & 24.0 & 34.0 & 27.0 & 12 & 24 & 34 & 27.0 & 12 & 24 & 34 & 27.0 \\
MReO & 16.4 & 25.8 & 35.5 & 40.0 & 10 & 17 & 25 & -6.8 & 32 & 42 & 53 & 128.4 \\
MReW & 17.9 & 28.0 & 38.4 & 51.7 & 11 & 18 & 26 & -1.4 & 39 & 48 & 68 & 174.3 \\
\hline
\end{tabular}


Table 9 - 11 Maximum fuzzy machine workload results of instance 2

\begin{tabular}{llllllllllllll}
\hline Algorithm & $\begin{array}{l}\text { Insertion } \\
\text { times }\end{array}$ & \multicolumn{3}{c}{ Average } & $\begin{array}{l}\text { Imp } \\
(\%)\end{array}$ & & Best & $\begin{array}{c}\text { Imp } \\
(\%)\end{array}$ & & Worst & $\begin{array}{c}\text { Imp } \\
(\%)\end{array}$ \\
\hline TFABC & 0 & 16.5 & 29.5 & 41.1 & & 13 & 30 & 41 & & 17 & 30 & 42 & \\
MinEnd & & 28.3 & 44.9 & 60.7 & 53.3 & 23 & 37 & 49 & 28.1 & 37 & 56 & 78 & 90.8 \\
GS & & 23.4 & 38.1 & 51.9 & 29.8 & 23 & 35 & 44 & 20.2 & 27 & 43 & 60 & 45.4 \\
LS & & 31.0 & 45.0 & 65.0 & 59.5 & 31 & 45 & 65 & 63.2 & 31 & 45 & 65 & 56.3 \\
MReO & 33.5 & 54.7 & 75.1 & 86.8 & 27 & 40 & 54 & 41.2 & 56 & 80 & 107 & 171.4 \\
MReW & & 37.7 & 57.7 & 79.6 & 99.6 & 22 & 36 & 49 & 25.4 & 67 & 91 & 126 & 215.1 \\
& & & & & & & & & & & & & \\
TFABC & 1 & 18.6 & 30.3 & 41.7 & & 17 & 26 & 37 & & 19 & 27 & 34 & \\
MinEnd & 23.3 & 37.4 & 50.3 & 22.9 & 19 & 31 & 40 & 14.2 & 32 & 48 & 64 & 79.4 \\
GS & 20.7 & 32.0 & 43.9 & 6.4 & 21 & 28 & 35 & 5.7 & 20 & 38 & 52 & 38.3 \\
LS & 23.0 & 35.0 & 52.0 & 20.0 & 23 & 35 & 52 & 36.8 & 23 & 35 & 52 & 35.5 \\
MReO & 33.3 & 50.5 & 68.9 & 68.2 & 17 & 31 & 44 & 16.0 & 65 & 86 & 113 & 227.1 \\
MReW & 29.9 & 49.1 & 66.9 & 61.5 & 20 & 36 & 52 & 35.8 & 55 & 80 & 107 & 200.9 \\
& & & & & & & & & & & & & \\
TFABC & 2 & 15.0 & 24.0 & 34.0 & & 15 & 24 & 34 & & 15 & 24 & 34 & \\
MinEnd & 22.1 & 34.9 & 47.4 & 43.6 & 18 & 29 & 42 & 21.6 & 29 & 46 & 64 & 90.7 \\
GS & 20.0 & 29.7 & 40.0 & 23.2 & 16 & 28 & 38 & 13.4 & 20 & 33 & 47 & 37.1 \\
LS & 27.0 & 39.0 & 57.0 & 67.0 & 27 & 39 & 57 & 67.0 & 27 & 39 & 57 & 67.0 \\
MReO & 29.6 & 46.8 & 64.4 & 93.4 & 20 & 32 & 44 & 32.0 & 50 & 78 & 108 & 223.7 \\
MReW & 31.3 & 51.3 & 70.6 & 110.7 & 21 & 35 & 48 & 43.3 & 64 & 93 & 122 & 283.5 \\
\hline
\end{tabular}

Table 9 - 12 Maximum fuzzy machine workload results of instance 3

\begin{tabular}{llllllllllllll}
\hline Algorithm & $\begin{array}{l}\text { Insertion } \\
\text { times }\end{array}$ & \multicolumn{3}{c}{ Average } & $\begin{array}{l}\text { Imp } \\
(\%)\end{array}$ & & Best & $\begin{array}{r}\text { Imp } \\
(\%)\end{array}$ & Worst & $\begin{array}{r}\text { Imp } \\
(\%)\end{array}$ \\
\hline TFABC & 0 & 32.3 & 53.3 & 73.1 & & 34 & 53 & 71 & & 36 & 54 & 70 & \\
MinEnd & & 46.0 & 72.5 & 97.2 & 36.0 & 40 & 60 & 83 & 15.2 & 55 & 87 & 115 & 60.7 \\
GS & & 41.7 & 67.1 & 90.4 & 25.7 & 40 & 61 & 83 & 16.1 & 49 & 74 & 96 & 36.9 \\
LS & & 51.0 & 76.0 & 102.0 & 43.9 & 51 & 76 & 102 & 44.5 & 51 & 76 & 102 & 42.5 \\
MReO & & 55.3 & 85.0 & 113.1 & 59.7 & 37 & 65 & 89 & 21.3 & 95 & 136 & 175 & 153.3 \\
MReW & & 54.3 & 84.1 & 112.8 & 58.2 & 40 & 62 & 85 & 18.0 & 99 & 130 & 169 & 146.7 \\
& & & & & & & & & & & & & \\
TFABC & 1 & 26.3 & 45.2 & 63.4 & & 27 & 45 & 62 & & 26 & 46 & 63 & \\
MinEnd & & 39.6 & 63.6 & 85.2 & 40.0 & 33 & 54 & 70 & 17.9 & 50 & 74 & 93 & 60.8 \\
GS & & 38.6 & 59.6 & 81.4 & 32.9 & 33 & 52 & 73 & 17.3 & 43 & 66 & 94 & 48.6 \\
LS & 47.0 & 73.0 & 100.0 & 62.8 & 47 & 73 & 100 & 63.7 & 47 & 73 & 100 & 61.9 \\
MReO & 49.0 & 75.5 & 100.9 & 67.2 & 30 & 52 & 71 & 14.5 & 89 & 121 & 154 & 168.0 \\
MReW & 47.8 & 77.1 & 103.2 & 69.5 & 37 & 57 & 79 & 28.5 & 91 & 127 & 165 & 181.8 \\
& & & & & & & & & & & & & \\
TFABC & 2 & 28.7 & 45.2 & 61.3 & & 31 & 45 & 59 & & 25 & 46 & 65 & \\
MinEnd & & 40.5 & 63.0 & 83.6 & 38.5 & 36 & 55 & 72 & 21.1 & 47 & 73 & 97 & 59.3 \\
GS & 37.7 & 57.4 & 78.2 & 27.8 & 30 & 51 & 73 & 13.9 & 44 & 68 & 96 & 51.6 \\
LS & 41.0 & 67.0 & 90.0 & 46.8 & 41 & 67 & 90 & 47.2 & 41 & 67 & 90 & 45.6 \\
\hline
\end{tabular}




\begin{tabular}{|c|c|c|c|c|c|c|c|c|c|c|c|c|c|}
\hline $\mathrm{MReO}$ & & 47.7 & 73.3 & 99.0 & 62.5 & 31 & 54 & 79 & 21.1 & 94 & 130 & 166 & 185.7 \\
\hline MReW & & 53.9 & 80.7 & 107.7 & 79.0 & 37 & 58 & 81 & 30.0 & 94 & 127 & 164 & 181.3 \\
\hline TFABC & 3 & 25.9 & 40.2 & 54.5 & & 22 & 40 & 57 & & 27 & 41 & 54 & \\
\hline MinEnd & & 38.2 & 59.9 & 79.5 & 47.7 & 34 & 50 & 63 & 23.9 & 49 & 71 & 94 & 74.8 \\
\hline GS & & 34.4 & 51.7 & 68.6 & 28.4 & 30 & 48 & 67 & 21.4 & 35 & 57 & 78 & 39.3 \\
\hline LS & & 38.0 & 59.0 & 77.0 & 44.9 & 38 & 59 & 77 & 46.5 & 38 & 59 & 77 & 42.9 \\
\hline $\mathrm{MReO}$ & & 47.4 & 72.6 & 96.5 & 79.7 & 34 & 52 & 68 & 29.6 & 80 & 116 & 147 & 181.6 \\
\hline MReW & & 48.1 & 72.2 & 96.2 & 79.5 & 32 & 51 & 70 & 28.3 & 83 & 116 & 154 & 187.7 \\
\hline
\end{tabular}

Table 9 - 13 Maximum fuzzy machine workload results of instance 4

\begin{tabular}{lllllllllllllrr}
\hline Algorithm & $\begin{array}{l}\text { Insertion } \\
\text { times }\end{array}$ & \multicolumn{3}{c}{ Average } & \multicolumn{1}{c}{$\begin{array}{l}\text { Imp } \\
(\%)\end{array}$} & & Best & & $\begin{array}{r}\text { Imp } \\
(\%)\end{array}$ & & Worst & $\begin{array}{r}\text { Imp } \\
(\%)\end{array}$ \\
\hline TFABC & 0 & 21.4 & 37.5 & 53.1 & & 28 & 36 & 48 & & 22 & 37 & 56 & \\
MinEnd & & 36.6 & 54.6 & 74.8 & 47.5 & 27 & 48 & 68 & 29.1 & 50 & 74 & 101 & 96.7 \\
GS & & 36.9 & 52.9 & 71.1 & 43.0 & 30 & 43 & 59 & 18.2 & 45 & 65 & 89 & 73.7 \\
LS & & 46.0 & 72.0 & 103.0 & 96.0 & 46 & 72 & 103 & 98.0 & 46 & 72 & 103 & 92.8 \\
MReO & 47.0 & 68.8 & 93.7 & 86.2 & 35 & 50 & 69 & 37.8 & 91 & 120 & 159 & 222.4 \\
MReW & 49.3 & 72.8 & 99.5 & 96.9 & 32 & 52 & 76 & 43.2 & 91 & 120 & 162 & 224.3 \\
& & & & & & & & & & & & & \\
TFABC & 1 & 24.5 & 36.4 & 49.7 & & 28 & 35 & 47 & & 23 & 36 & 53 & \\
MinEnd & 37.1 & 54.2 & 73.9 & 49.2 & 35 & 47 & 63 & 32.4 & 45 & 65 & 87 & 77.0 \\
GS & & 29.3 & 44.3 & 59.2 & 20.5 & 25 & 39 & 55 & 9.0 & 34 & 49 & 62 & 31.1 \\
LS & 44.0 & 67.0 & 96.0 & 86.3 & 44 & 67 & 96 & 89.0 & 44 & 67 & 96 & 85.1 \\
MReO & 55.4 & 81.5 & 111.6 & 124.4 & 42 & 53 & 71 & 51.0 & 69 & 109 & 153 & 197.3 \\
MReW & 53.8 & 78.1 & 105.7 & 114.6 & 34 & 49 & 67 & 37.2 & 116 & 157 & 207 & 330.4 \\
& & & & & & & & & & & & & \\
TFABC & 2 & 19.2 & 32.7 & 44.6 & & 19 & 32 & 45 & & 17 & 33 & 49 & \\
MinEnd & 34.1 & 51.8 & 67.9 & 59.0 & 25 & 41 & 57 & 28.1 & 39 & 60 & 79 & 80.3 \\
GS & 30.5 & 46.0 & 61.3 & 42.2 & 27 & 40 & 53 & 25.0 & 39 & 55 & 72 & 67.4 \\
LS & 46.0 & 71.0 & 99.0 & 122.0 & 46 & 71 & 99 & 124.2 & 46 & 71 & 99 & 117.4 \\
MReO & 55.6 & 80.7 & 109.0 & 152.1 & 44 & 60 & 78 & 89.1 & 95 & 134 & 178 & 309.8 \\
MReW & 44.1 & 70.5 & 95.7 & 117.3 & 28 & 52 & 67 & 55.5 & 79 & 106 & 147 & 231.8 \\
\hline
\end{tabular}

Table 9 - 14 Maximum fuzzy machine workload results of instance 5

\begin{tabular}{llrrrrrrrrrrrr}
\hline Algorithm & $\begin{array}{l}\text { Insertion } \\
\text { times }\end{array}$ & \multicolumn{3}{c}{ Average } & \multicolumn{1}{c}{$\begin{array}{l}\text { Imp } \\
(\%)\end{array}$} & & Best & \multicolumn{1}{c}{$\begin{array}{c}\text { Imp } \\
(\%)\end{array}$} & \multicolumn{2}{c}{ Worst } & $\begin{array}{r}\text { Imp } \\
(\%)\end{array}$ \\
\hline TFABC & 0 & 43.5 & 74.4 & 104.9 & & 36 & 75 & 109 & & 43 & 74 & 108 & \\
MinEnd & & 63.7 & 102.9 & 140.9 & 38.1 & 59 & 92 & 121 & 23.4 & 73 & 110 & 153 & 49.2 \\
GS & & 55.8 & 89.8 & 123.7 & 20.8 & 51 & 85 & 121 & 15.9 & 57 & 96 & 133 & 27.8 \\
LS & & 72.0 & 101.0 & 137.0 & 38.3 & 72 & 101 & 137 & 39.3 & 72 & 101 & 137 & 37.5 \\
MReO & 86.4 & 136.3 & 184.2 & 82.8 & 61 & 104 & 147 & 41.0 & 133 & 186 & 251 & 152.8 \\
MReW & 82.8 & 133.7 & 181.1 & 78.7 & 64 & 98 & 137 & 34.6 & 140 & 202 & 267 & 171.2 \\
& & & & & & & & & & & & & \\
TFABC & 1 & 37.1 & 65.8 & 92.5 & & 42 & 64 & 90 & & 40 & 65 & 93 & \\
\hline
\end{tabular}




\begin{tabular}{lrrrrrrrrrrrr}
\hline MinEnd & 57.8 & 93.0 & 128.1 & 42.4 & 50 & 83 & 113 & 26.5 & 71 & 109 & 149 & 66.5 \\
GS & 49.8 & 82.1 & 112.3 & 24.9 & 44 & 78 & 110 & 19.2 & 55 & 87 & 117 & 31.6 \\
LS & 56.0 & 92.0 & 123.0 & 38.9 & 56 & 92 & 123 & 39.6 & 56 & 92 & 123 & 38.0 \\
MReO & 87.8 & 132.9 & 178.7 & 103.7 & 51 & 96 & 137 & 46.2 & 135 & 181 & 240 & 180.2 \\
MReW & 92.2 & 141.5 & 189.8 & 116.2 & 62 & 103 & 137 & 55.8 & 129 & 192 & 261 & 194.3 \\
& & & & & & & & & & & & \\
TFABC & 2 & 34.6 & 57.9 & 80.3 & & 36 & 57 & 79 & & 38 & 57 & 81 \\
MinEnd & 47.8 & 75.5 & 102.5 & 30.6 & 45 & 69 & 93 & 20.5 & 49 & 82 & 115 & 40.8 \\
GS & 48.3 & 77.3 & 104.6 & 33.2 & 49 & 72 & 97 & 26.6 & 50 & 82 & 111 & 39.5 \\
LS & 53.0 & 95.0 & 121.0 & 57.7 & 53 & 95 & 121 & 59.0 & 53 & 95 & 121 & 56.2 \\
MReO & 68.5 & 109.1 & 146.5 & 87.7 & 52 & 84 & 112 & 45.0 & 121 & 164 & 219 & 186.7 \\
MReW & 86.3 & 133.1 & 179.4 & 130.5 & 57 & 88 & 115 & 52.0 & 133 & 184 & 240 & 218.0 \\
\hline
\end{tabular}

Table 9 - 15 Maximum fuzzy machine workload results of instance 6

\begin{tabular}{llrrrrrrrrrrrr}
\hline Algorithm & $\begin{array}{l}\text { Insertion } \\
\text { times }\end{array}$ & \multicolumn{3}{c}{ Average } & & \multicolumn{1}{c}{$\begin{array}{l}\text { Imp } \\
(\%)\end{array}$} & & Best & & $\begin{array}{r}\text { Imp } \\
(\%)\end{array}$ & Worst & $\begin{array}{r}\text { Imp } \\
(\%)\end{array}$ \\
\hline TFABC & 0 & 34.5 & 62.0 & 86.2 & & 30 & 62 & 87 & & 38 & 63 & 85 & \\
MinEnd & & 52.2 & 86.7 & 118.1 & 40.4 & 50 & 78 & 106 & 29.5 & 60 & 101 & 139 & 61.0 \\
GS & & 45.9 & 76.6 & 104.5 & 24.0 & 40 & 72 & 99 & 17.4 & 54 & 84 & 111 & 33.7 \\
LS & & 56.0 & 88.0 & 119.0 & 43.4 & 56 & 88 & 119 & 45.6 & 56 & 88 & 119 & 41.0 \\
MReO & & 74.0 & 113.8 & 153.1 & 85.8 & 51 & 85 & 112 & 38.2 & 109 & 160 & 217 & 159.4 \\
MReW & & 74.5 & 116.2 & 156.8 & 89.5 & 51 & 80 & 111 & 33.6 & 134 & 181 & 237 & 194.4 \\
& & & & & & & & & & & & & \\
TFABC & 1 & 30.8 & 57.4 & 81.1 & & 29 & 58 & 80 & & 32 & 58 & 81 & \\
MinEnd & & 48.8 & 79.7 & 110.0 & 40.3 & 40 & 71 & 102 & 26.2 & 62 & 100 & 134 & 72.9 \\
GS & & 41.6 & 71.0 & 97.5 & 24.0 & 38 & 67 & 89 & 16.0 & 47 & 77 & 105 & 33.6 \\
LS & 47.0 & 85.0 & 121.0 & 49.1 & 47 & 85 & 121 & 50.2 & 47 & 85 & 121 & 47.6 \\
MReO & 71.3 & 112.7 & 153.6 & 98.6 & 48 & 83 & 117 & 47.1 & 138 & 191 & 249 & 235.8 \\
MReW & 73.7 & 117.2 & 159.7 & 106.2 & 54 & 84 & 109 & 47.1 & 127 & 183 & 242 & 221.0 \\
& & & & & & & & & & & & & \\
TFABC & 2 & 28.5 & 54.1 & 76.7 & & 30 & 54 & 74 & & 30 & 55 & 75 & \\
MinEnd & 44.1 & 72.5 & 99.7 & 35.2 & 38 & 62 & 85 & 16.5 & 57 & 82 & 112 & 54.9 \\
GS & 37.8 & 62.3 & 85.6 & 16.2 & 34 & 56 & 77 & 5.2 & 44 & 69 & 91 & 27.0 \\
LS & 51.0 & 88.0 & 124.0 & 64.5 & 51 & 88 & 124 & 65.6 & 51 & 88 & 124 & 63.3 \\
MReO & 70.6 & 108.4 & 146.9 & 103.5 & 42 & 72 & 101 & 35.4 & 112 & 145 & 189 & 174.9 \\
MReW & 76.0 & 121.3 & 166.1 & 127.1 & 50 & 98 & 134 & 79.2 & 110 & 155 & 204 & 190.2 \\
& & & & & & & & & & & \\
TFABC & 3 & 25.7 & 49.4 & 70.9 & & 23 & 49 & 73 & & 26 & 49 & 74 & \\
MinEnd & 38.1 & 66.8 & 92.2 & 35.1 & 31 & 60 & 88 & 23.2 & 41 & 75 & 103 & 48.5 \\
GS & 34.5 & 59.7 & 82.9 & 21.2 & 34 & 57 & 78 & 16.5 & 39 & 65 & 87 & 29.3 \\
LS & 53.0 & 90.0 & 131.0 & 86.3 & 53 & 90 & 131 & 87.6 & 53 & 90 & 131 & 83.8 \\
MReO & 53.3 & 90.5 & 128.1 & 85.5 & 46 & 70 & 98 & 46.4 & 84 & 126 & 168 & 154.5 \\
MReW & 76.6 & 122.6 & 167.1 & 150.2 & 40 & 85 & 121 & 70.6 & 119 & 170 & 222 & 243.9 \\
\hline & & & & & & & & & & & &
\end{tabular}


Table 9 - 16 Maximum fuzzy machine workload results of instance 7

\begin{tabular}{llcccccccccccc}
\hline Algorithm & $\begin{array}{l}\text { Insertion } \\
\text { times }\end{array}$ & \multicolumn{3}{c}{ Average } & & $\begin{array}{c}\text { Imp } \\
(\%)\end{array}$ & & Best & & $\begin{array}{c}\text { Imp } \\
(\%)\end{array}$ & Worst & $\begin{array}{l}\text { Imp } \\
(\%)\end{array}$ \\
\hline TFABC & 0 & 50.5 & 97.0 & 138.5 & & 49 & 97 & 137 & & 50 & 97 & 145 & \\
MinEnd & & 74.6 & 130.3 & 183.1 & 35.3 & 68 & 119 & 165 & 23.9 & 76 & 143 & 201 & 44.7 \\
GS & & 68.1 & 117.8 & 165.7 & 22.5 & 64 & 112 & 159 & 17.6 & 73 & 123 & 172 & 26.2 \\
LS & & 66.0 & 122.0 & 172.0 & 25.8 & 66 & 122 & 172 & 26.8 & 66 & 122 & 172 & 23.9 \\
MReO & 127.1 & 201.0 & 275.5 & 110.1 & 81 & 143 & 193 & 47.4 & 205 & 307 & 399 & 213.1 \\
MReW & 102.9 & 177.7 & 245.5 & 83.7 & 78 & 142 & 200 & 47.9 & 165 & 236 & 310 & 143.4 \\
& & & & & & & & & & & & & \\
TFABC & 1 & 49.9 & 95.6 & 136.2 & & 46 & 94 & 140 & & 48 & 96 & 141 & \\
MinEnd & & 76.4 & 129.1 & 178.7 & 36.0 & 68 & 121 & 165 & 27.0 & 87 & 140 & 192 & 46.7 \\
GS & 65.5 & 116.1 & 163.2 & 22.1 & 56 & 110 & 164 & 17.6 & 60 & 122 & 178 & 26.5 \\
LS & & 70.0 & 123.0 & 163.0 & 26.9 & 70 & 123 & 163 & 28.1 & 70 & 123 & 163 & 25.7 \\
MReO & 111.1 & 188.3 & 262.3 & 98.8 & 75 & 144 & 205 & 51.9 & 166 & 247 & 337 & 161.7 \\
MReW & 108.0 & 180.3 & 247.9 & 89.9 & 71 & 137 & 201 & 46.0 & 179 & 251 & 333 & 166.1 \\
& & & & & & & & & & & & \\
TFABC & 2 & 42.4 & 80.5 & 115.2 & & 42 & 80 & 112 & & 47 & 80 & 118 & \\
MinEnd & 65.7 & 112.6 & 156.5 & 40.4 & 63 & 106 & 144 & 33.4 & 74 & 119 & 169 & 48.0 \\
GS & 63.0 & 104.6 & 146.1 & 31.2 & 51 & 100 & 141 & 24.8 & 72 & 110 & 153 & 36.9 \\
LS & 63.0 & 118.0 & 166.0 & 45.9 & 63 & 118 & 166 & 48.1 & 63 & 118 & 166 & 43.1 \\
MReO & 114.6 & 182.4 & 245.7 & 127.6 & 73 & 137 & 189 & 70.7 & 155 & 225 & 299 & 178.2 \\
MReW & 97.7 & 166.1 & 225.6 & 105.7 & 66 & 121 & 171 & 52.5 & 173 & 262 & 345 & 220.6 \\
\hline
\end{tabular}

Table 9 - 17 Maximum fuzzy machine workload results of instance 8

\begin{tabular}{llllllllllllll}
\hline Algorithm & $\begin{array}{l}\text { Insertion } \\
\text { times }\end{array}$ & \multicolumn{3}{c}{ Average } & & $\begin{array}{l}\text { Imp } \\
(\%)\end{array}$ & & Best & & $\begin{array}{c}\text { Imp } \\
(\%)\end{array}$ & Worst & $\begin{array}{c}\text { Imp } \\
(\%)\end{array}$ \\
\hline TFABC & 0 & 40.6 & 75.3 & 106.8 & & 42 & 74 & 101 & & 44 & 77 & 107 & \\
MinEnd & & 58.2 & 98.9 & 136.7 & 31.8 & 55 & 90 & 122 & 22.7 & 69 & 109 & 148 & 42.6 \\
GS & & 50.2 & 86.4 & 120.1 & 15.1 & 48 & 84 & 114 & 13.4 & 57 & 91 & 125 & 19.3 \\
LS & & 62.0 & 101.0 & 139.0 & 35.3 & 62 & 101 & 139 & 38.5 & 62 & 101 & 139 & 32.1 \\
MReO & 87.9 & 145.4 & 198.9 & 93.9 & 58 & 109 & 154 & 47.8 & 161 & 233 & 315 & 208.9 \\
MReW & & 99.7 & 158.2 & 212.8 & 111.1 & 61 & 115 & 153 & 52.6 & 148 & 203 & 266 & 168.9 \\
& & & & & & & & & & & & & \\
TFABC & 1 & 38.3 & 72.7 & 103.9 & & 38 & 70 & 102 & & 37 & 74 & 109 & \\
MinEnd & 54.7 & 93.4 & 128.4 & 28.7 & 50 & 85 & 116 & 20.0 & 52 & 104 & 144 & 37.4 \\
GS & & 47.7 & 84.7 & 117.6 & 16.4 & 44 & 78 & 119 & 13.9 & 52 & 90 & 130 & 23.1 \\
LS & 61.0 & 107.0 & 149.0 & 47.5 & 61 & 107 & 149 & 51.4 & 61 & 107 & 149 & 44.2 \\
MReO & 96.3 & 154.1 & 208.9 & 113.3 & 60 & 111 & 151 & 54.6 & 147 & 208 & 272 & 184.0 \\
MReW & 103.3 & 167.3 & 227.6 & 131.5 & 63 & 115 & 166 & 63.9 & 171 & 250 & 323 & 238.1 \\
& & & & & & & & & & & & & \\
TFABC & 2 & 36.8 & 69.7 & 101.0 & & 37 & 69 & 96 & & 37 & 71 & 103 & \\
MinEnd & 56.3 & 92.9 & 127.4 & 33.2 & 54 & 88 & 116 & 27.7 & 63 & 102 & 138 & 43.6 \\
GS & 49.9 & 84.1 & 115.2 & 20.2 & 44 & 81 & 109 & 16.2 & 56 & 89 & 121 & 25.9 \\
LS & 51.0 & 88.0 & 121.0 & 25.5 & 51 & 88 & 121 & 28.4 & 51 & 88 & 121 & 23.4 \\
\hline
\end{tabular}




\begin{tabular}{|c|c|c|c|c|c|c|c|c|c|c|c|c|c|}
\hline $\mathrm{MReO}$ & & 91.8 & 148.3 & 202.3 & 113.0 & 62 & 103 & 135 & 48.7 & 150 & 222 & 299 & 216.7 \\
\hline MReW & & 94.5 & 160.7 & 221.1 & 129.7 & 57 & 122 & 175 & 75.6 & 156 & 222 & 295 & 217.4 \\
\hline TFABC & 3 & 34.9 & 64.7 & 91.7 & & 38 & 63 & 89 & & 38 & 66 & 92 & \\
\hline MinEnd & & 49.7 & 85.0 & 117.3 & 31.6 & 45 & 80 & 106 & 22.9 & 53 & 93 & 136 & 43.1 \\
\hline GS & & 45.5 & 77.6 & 107.0 & 20.1 & 40 & 74 & 101 & 14.2 & 48 & 82 & 114 & 24.4 \\
\hline LS & & 47.0 & 81.0 & 111.0 & 4.0 & 47 & 81 & 111 & 26.5 & 47 & 81 & 111 & 22.1 \\
\hline $\mathrm{MReO}$ & & 78.0 & 131.7 & 181.5 & 70.0 & 47 & 104 & 151 & 60.5 & 127 & 182 & 249 & 182.4 \\
\hline MReW & & 93.8 & 161.3 & 223.7 & 108.1 & 58 & 115 & 157 & 75.9 & 178 & 256 & 334 & 290.8 \\
\hline
\end{tabular}

Table 9 - 18 TFABC algorithm's average CPU times for all instances and new job insertions

\begin{tabular}{ccccccccc}
\hline Instance & $\begin{array}{c}\text { Insertio } \\
\mathrm{n} \\
\text { times }\end{array}$ & $\begin{array}{c}\text { CPU } \\
\text { Time } \\
(\mathrm{s})\end{array}$ & Instance & $\begin{array}{c}\text { Insertio } \\
\mathrm{n} \\
\text { times }\end{array}$ & $\begin{array}{c}\text { CPU } \\
\text { Time } \\
(\mathrm{s})\end{array}$ & Instance & $\begin{array}{c}\text { Insertio } \\
\mathrm{n} \\
\text { times }\end{array}$ & $\begin{array}{c}\text { CPU } \\
\text { Time } \\
(\mathrm{s})\end{array}$ \\
\hline instance & & & instance & & & instance & & \\
1 & 0 & 0.474 & 4 & 0 & 3.302 & 7 & 0 & 66.605 \\
& 1 & 0.403 & & 1 & 3.024 & & 1 & 60.380 \\
& 2 & 0.400 & & 2 & 2.699 & & 2 & 43.889 \\
instance & & & instance & & & instance & & \\
2 & 0 & 1.748 & 5 & 0 & 25.266 & 8 & 0 & 107.540 \\
& 1 & 1.443 & & 1 & 20.260 & & 1 & 98.295 \\
& 2 & 1.105 & & 2 & 15.394 & & 2 & 86.357 \\
instance & & & instance & & & & & \\
3 & 0 & 2.222 & 6 & 0 & 29.878 & & 3 & 70.815 \\
& 1 & 1.767 & & 1 & 27.350 & & & \\
& 2 & 1.628 & & 2 & 24.690 & & & \\
\hline
\end{tabular}




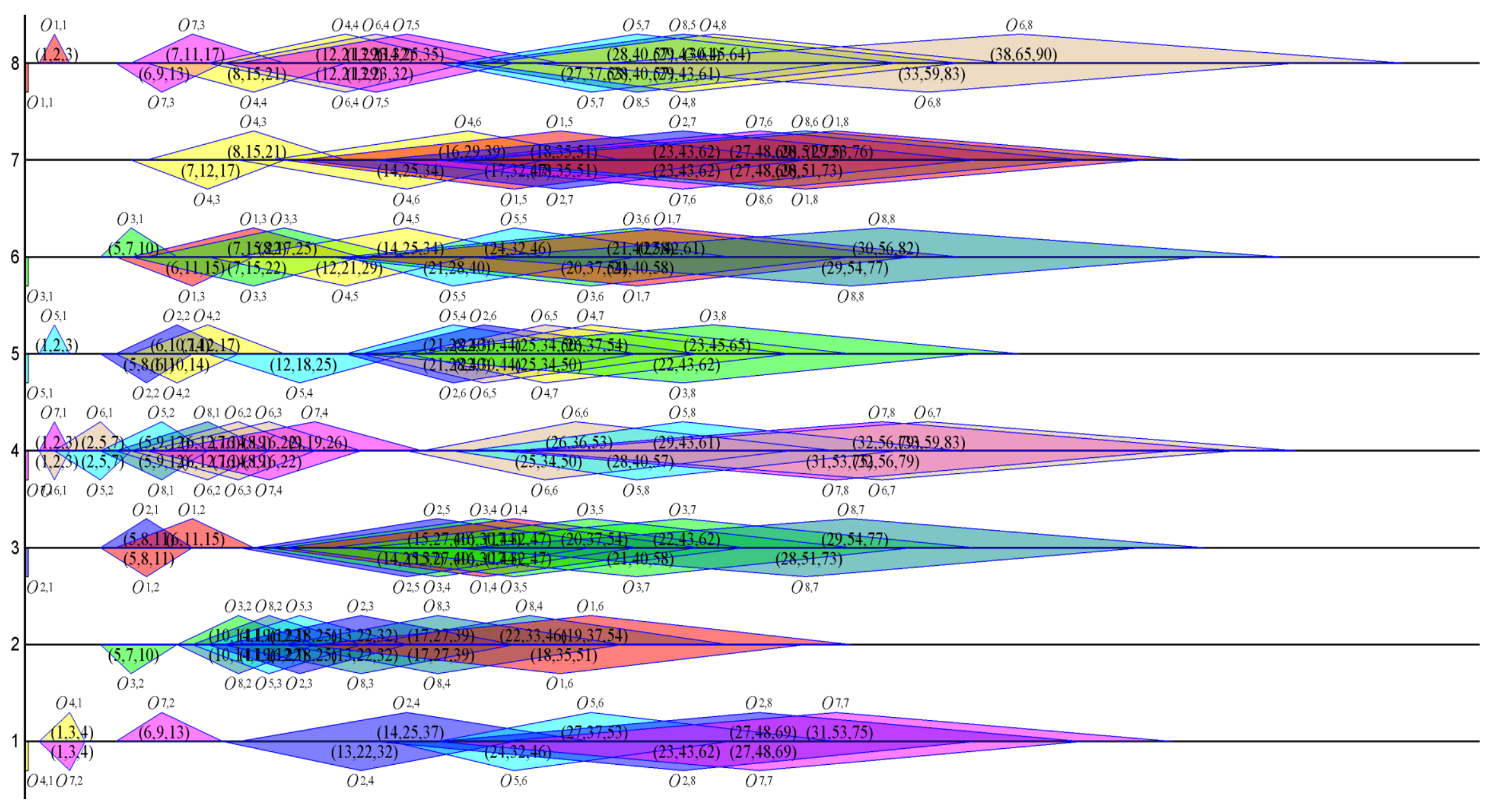

Fig. 9 - 7 Gantt chart of instance Reman 2 with minimum machine workload 


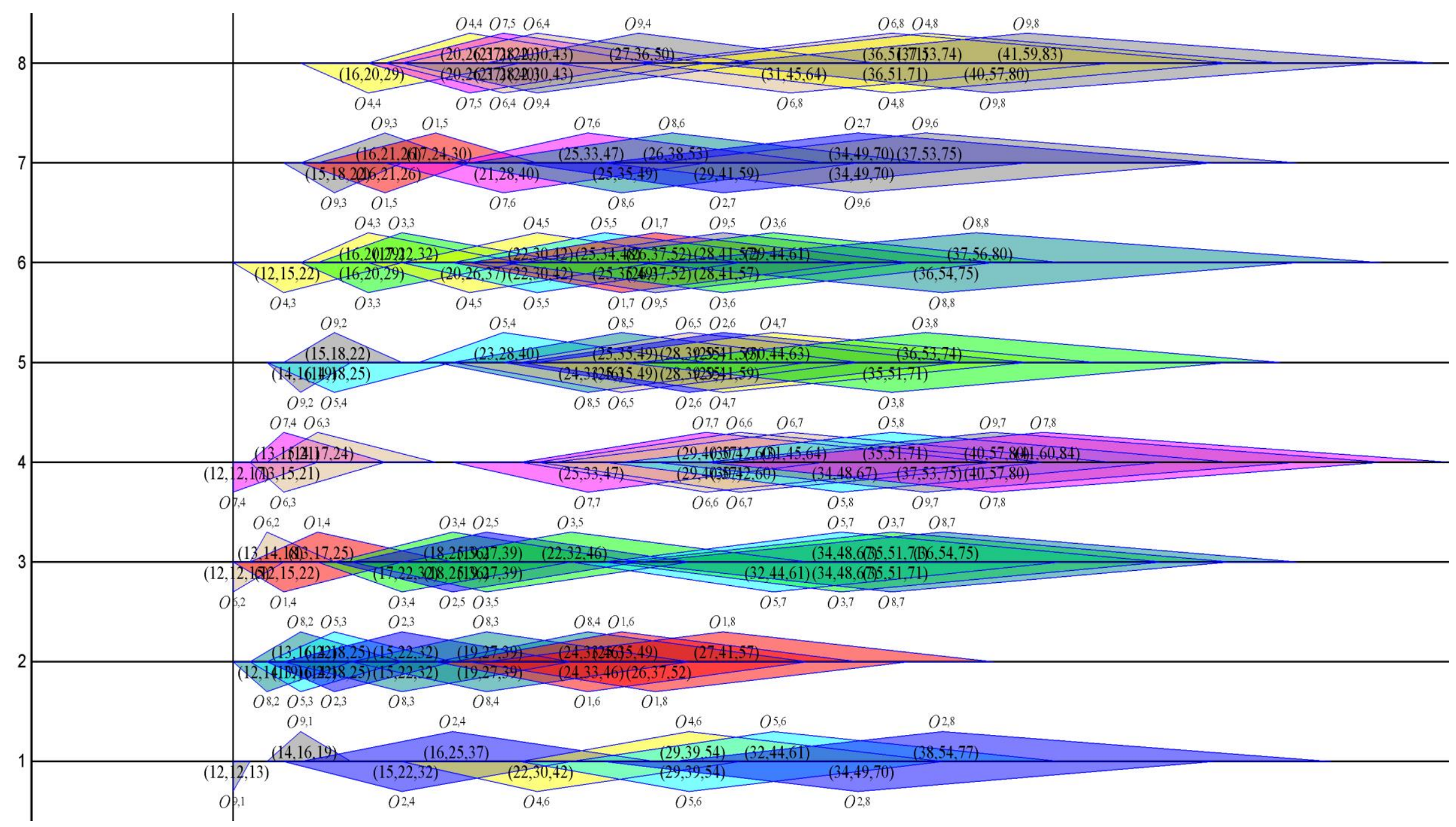

Fig. 9 - 8 First rescheduling Gantt chart with best fuzzy machine workload 


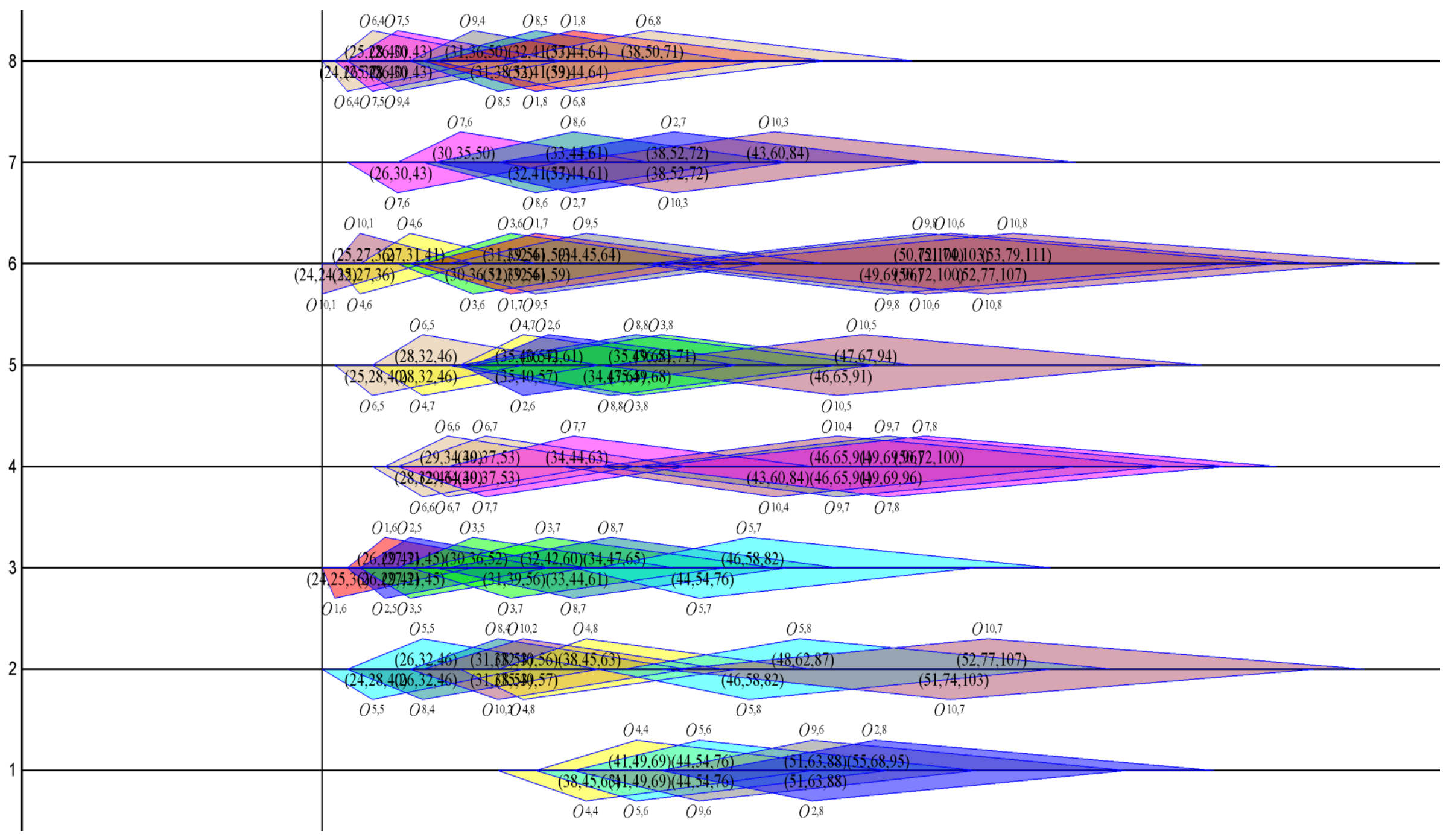

Fig. 9 - 9 Second rescheduling Gantt chart with best fuzzy machine workload 


\subsection{Conclusions}

In this Chapter, a two-stage fuzzy artificial bee colony (TFABC) algorithm was proposed to solve flexible job shop scheduling problem with fuzzy processing time and new job(s) insertion constraints. The objectives were to minimize the maximum fuzzy completion time and the maximum fuzzy machine workload. The MinEnd heuristic and global minimum processing time heuristic were employed to initialize population. To test the performance, the TFABC was compared to five heuristics for solving eight instances from remanufacturing industry. The results and comparisons demonstrated the performance of TFABC algorithm. The TFABC algorithm improved the results of heuristics obviously. TFABC algorithm cost very low computer CPU time. In addition, several fuzzy Gantt charts of best solution were shown for the maximum fuzzy completion time and the maximum fuzzy machine workload objectives. 


\section{Chapter 10}

\section{Conclusions and Future Work}

\subsection{Conclusions}

This thesis researched on multi-objective multi-constraint flexible job shop scheduling problem (FJSSP). Two scheduling related remanufacturing characteristics, namely uncertain processing time and new job insertion, were modeled as two constraints of FJSSP. The scheduling objectives were to minimize maximum completion time (makespan), maximum machine workload, mean of earliness and tardiness. The major contributions of this thesis are shown as follows.

- Two strategies, most probable processing time and fuzzy processing time, were employed to represent the uncertain processing time of operations in remanufacturing industry. One operation had two processing times. The first one was the most probable processing time and the second one was a fuzzy processing time that was represented by a triangle fuzzy number. Rescheduling was executed to deal with new job(s) insertion constraint.

- To solve general FJSSP, a discrete harmony search algorithm was proposed. In the DHS algorithm, several heuristics were used to initialize the population. Dynamic grouping, local search methods were developed to improve the algorithm performance. To test the performance, the DHS algorithm was employed to solve benchmark instances and was compared to several existing competitive algorithms.

- To solve FJSSP with most probable processing time and new job insertion constraints, we proposed a two-stage artificial bee colony algorithm. Three rescheduling strategies were proposed for rescheduling when new job arrived and was inserted into existing scheduling solution. The 
three rescheduling strategies were evaluated and compared. To test the performance of the twostage $\mathrm{ABC}$ algorithm, the scheduling and rescheduling results were compared to other heuristics and composite heuristics.

- A two-stage DHS (TDHS) algorithm was proposed to solve FJSSP with two constraints, processing time increasing and new job insertion. Two real remanufacturing instances were tested using the TDHS algorithm. Rescheduling was executed for both of most probable processing time increasing and new job insertion. The scheduling and rescheduling results could satisfy practical remanufacturing process.

- To solve FJSSP with fuzzy processing time, a fuzzy DHS (FDHS) algorithm was proposed. One heuristic, named MinEnd, was proposed to improve the quality of initial population. To evaluate performance, the FDHS algorithm was compared to five existing competitive algorithms for solving benchmark instances. Eight instances from remanufacturing industry were solved by the FDHS algorithm. The results were satisfactory for the practical process in the shop floor.

- To solve FJSSP with fuzzy processing time and new job insertion constraints, we proposed a fuzzy $\mathrm{ABC}(\mathrm{FABC})$ algorithm and a two-stage fuzzy $\mathrm{ABC}$ algorithm. The objectives were to minimize the maximum fuzzy completion time and maximum fuzzy machine workload. The FABC algorithm was compared to FDHS algorithm and five heuristics for solving eight remanufacturing instances. The two-stage FABC algorithm was compared to five heuristics for solving eight instances with new job insertion.

In summary, this thesis focused on scheduling problem in remanufacturing industry. The problem was modeled as FJSSP with two constraints. Two meta-heuristics, harmony search algorithm and artificial bee colony algorithm were employed and improved to solve the FJSSP with remanufacturing constraints. Benchmark instances and real instances from remanufacturing were solved. The experiments and comparisons showed the effectiveness and efficiency of proposed algorithms. The remanufacturing scheduling problem was solved satisfactorily. The proposed algorithms had been integrated to existing commercial planning and scheduling systems to improve the scheduling results.

\subsection{Future work}

Flexible job shop scheduling problem (FJSSP) is widespread in many fields. In future, we will focus on the following topics:

- We will research on more effective and efficient ensemble heuristics and meta-heuristics to solve the scheduling and rescheduling problem in remanufacturing industry. We will improve the convergence capability of the proposed DHS and ABC algorithms by considering additional local 
search methods. These algorithms will be used to solve dynamic flexible job shop scheduling problems and other classes of scheduling problems.

- We will address more scheduling objectives in remanufacturing environment, for example cost related objectives and a hybrid of different type's objectives. Weighted multi-objective and Pareto-based multi-objective will be considered in different scheduling and rescheduling problems.

- More general practical constraints will be considered in future research, for example, machine setup time, machine breakdown, maintenance activity, limited resources, overlapping operations, sequence-dependent setup and transportation times and so on.

- We will use our scheduling algorithm to solve more scheduling problems with practical constraints in different fields. We will co-operate scheduling solution for flexible job shop scheduling problem with processing time uncertainty, in addition to new job insertion. We will also consider other meta-heuristics and propose new heuristics for more scheduling problems in remanufacturing industry.

- Beside harmony search and artificial bee colony algorithms, we will research on new metaheuristics, for example, teaching learning based optimization. We will also employ the new metaheuristics for solving job shop scheduling problem, flow shop scheduling problem and scheduling problem in different fields. 


\section{Author's Publications}

\section{Journal papers (Accepted and Published):}

- $\quad$ K.Z. Gao, P.N. Suganthan, M.F. Tasgetiren, Q.K. Pan, et al. Effective ensembles of heuristics for scheduling flexible job shop problem with new job insertion. Computers \& Industrial Engineering, 2015, 90: 107-117.

- K.Z. Gao, P.N. Suganthan, Q.K. Pan, M.F. Tasgetiren. An effective discrete harmony search algorithm for flexible job shop scheduling problem with fuzzy processing time, International Journal of Production Research, 2015, 53(19): 5896-5911.

- $\quad$ K.Z. Gao, P.N. Suganthan, T.J. Chua, et al. A two-stage artificial bee colony algorithm scheduling flexible job-shop scheduling problem with new job insertion, Expert Systems with Applications, 2015, 42: 7652-7663.

1. K.Z. Gao, P.N. Suganthan, Q.K. Pan, T.J. Chua, et al. Pareto-based Grouping Discrete Harmony Search Algorithm for Multi-objective Flexible Job Shop Scheduling, Information Sciences, 2014, 289: 76-90.

2. K.Z. Gao, P.N. Suganthan, Q.K. Pan, T.J. Chua, et al. Discrete Harmony Search Algorithm for Flexible Job Shop Scheduling Problem with Weighted Combination of Multiple Objectives, Journal of Intelligence Manufacturing, Published online.

3. K.Z. Gao, P.N. Suganthan, T.J. Chua, Discrete harmony search algorithm for the disassembly scheduling in remanufacturing engineering, Applied Mechanics and Materials, 236-237: 169-174, 2012.

\section{Conference papers:}

1. K.Z. Gao, P.N. Suganthan, T.J. Chua, et al. Hybrid discrete harmony search algorithm for scheduling re-processing problem in remanufacturing, Proceeding of the fifteenth annual conference on Genetic and evolutionary computation conference (GECCO), page 1261-1268, Amsterdam, Netherlands, July 06-10, 2013.

2. Kai Zhou Gao, Ponnuthurai Nagaratnam Suganthan, Tay Jin Chua, Discrete harmony search algorithm for the dynamic FJSSP in remanufacturing engineering, Lecture Notes in Computer Science, 7677: 9-16, 2012.

3. Kai Zhou Gao, Ponnuthurai Nagaratnam Suganthan, Tay Jin Chua, Pareto-based discrete harmony search algorithm for flexible job shop scheduling, Proceeding of the 12th conference on 
Intelligent Systems, Design and Applications (ISDA), page 953-956, Kochi, India, Nov 27-29, 2012.

\section{Submitted papers:}

1. Kai Zhou Gao, Ponnuthurai Nagaratnam Suganthan, Tay Jin Chua, et al. An improved artificial bee colony algorithm for multi-objective flexible job shop scheduling problem with fuzzy processing time. Submitted to "Applied Soft Computing” of the Journal (Near accepted). 


\section{Bibliography}

[1] Lund R. I., Remanufacturing. Technology Review, 87 (1984) 18-23.

[2] Krupp J. A., Core obsolescence forecasting in remanufacturing. Production and Inventory Management Journal, 33 (1992) 12-17.

[3] Krupp J. A., Structuring bills of material for automotive remanufacturing. Production and Inventory Management Journal, 34 (1993) 46-52.

[4] DePuy G.W., Usher J.S., Walker R. L., Taylor G.D., Production planning for remanufactured products. Production Planning \& Control, 18 (2007) 573-583.

[5] Guide J., Souza G.C.,Van L., Performance of static priority rules for shared facilities in a remanufacturing shop with disassembly and reassembly. European Journal of Operational Research, 164 (2005) 341-353.

[6] Guide J., V D.R., Srivastava.R., Spencer M.S., An evaluation of capacity planning techniques in a remanufacturing environment. International Journal of Production Research, 35 (1997) 67-82.

[7] Guide J., V D.R., Srivastava R., Kraus M.E., Product structure complexity and scheduling of operations in recoverable manufacturing. International Journal of Production Research, 35 (1997) 3179-3200.

[8] Teunter R., Tang O., Kaparis K., Heuristics for the economic lot scheduling problem with returns. International Journal of Production Economics, 118 (2009) 323-330.

[9] Grubbström R.W., Tang O., Optimal production opportunities in a remanufacturing system. International Journal of Production Research, 44 (2006) 3953-3966.

[10] Daniel V. R., Guide J., Production planning and control for remanufacturing: industry practice and research needs. Journal of Operations Management, 18 (2000) 467-483.

[11] Muris L. J., Moacir G. F., Production planning and control for remanufacturing: literature review and analysis. Production Planning \& Control, 23 (2012) 419-435.

[12] Pinedo M, Scheduling: theory, algorithms, and systems. Prentice-Hall, Englewood cliffs, 2012.

[13]Garey M.R., Johnson D.S., Sethi R., The complexity of flow hop and job shop scheduling, Mathematics of Operations Research, 1 (1976) 117-129. 
[14]Kacem I., Hammadi S., Borne P., Approach by localization and multi-objective evolutionary optimization for flexible job shop scheduling problems, IEEE transaction on system, Man, and Cybernetics, 32 (2002) 113.

[15]Brucker P., Schlie R., Job-shop scheduling with multi-purpose machines, Computing, 45(1990) 369-375.

[16]Pezzella F., Morganti G., Ciaschetti G., A genetic algorithm for the flexible job-shop scheduling problem, Computers and Operations Research, 35 (2008) 3202-3212.

[17] Chu C, Portmann M C, Proth J M. A splitting-up approach to simplify job-shop scheduling problems. International Journal of Production Research, 30 (1992) 859-870.

[18] Balas E. Machine scheduling via disjunctive graphs: an implicit enumeration algorithm. Operations Research, 17 (1969) 941-957.

[19] Smith W. E. Various optimizers for single stage production. Naval Research Logistics Quarterly, 3 (1956) 59-66.

[20] Panwalker S S, Iskander W. A survey of scheduling rules. Operations Research, 25 (1977) 45-61.

[21]Wang Y.M., Ong H., L. Yin, Qin K.D., A novel genetic algorithm for flexible job shop scheduling problems with machine discruption, International Journal of Advanced Manufacturing Technology, 68 (2013) 13171326.

[22]Xing L.N., Chen Y.W., Wang P. etc. al, A knowledge-based ant colony optimization for flexible job shop scheduling problems, Applied Soft Computing, 10 (2010) 888-896.

[23]Xia W.J., Wu Z.M., An effective hybrid optimization approach for multi-objective flexible job-shop scheduling problems, Computers and Industrial Engineering, 48 (2005) 409-425.

[24] Yuan Y., Xu H., Flexible job shop scheduling using hybrid differential evolution algorithms, Computers and Industrial Engineering, 65 (2013) 246-260.

[25]Gao K.Z., Suganthan P.N., Pan Q.K., etc.al, Discrete harmony search algorithm for flexible job shop scheduling problem with multiple objectives, Journal of Intelligence Manufacturing, DOI: 10.1007/s10845014-0869-8.

[26] Li J.Q., Pan Q.K., Gao K.Z., Pareto-based discrete artificial bee colony algorithm for multi-objective flexible job shop scheduling problems, International Journal of Advanced Manufacturing Technology, 55 (2011) 1159-1169.

[27]Zhang G.H., Shao X.Y., Li P.G., Gao L., An effective hybrid particle swarm optimization algorithm for multi-objective flexible job-shop scheduling problem, Computers and Industrial Engineering, 56 (2009) $1309-1318$.

[28] Gao J., Sun L., Gen M., A hybrid genetic and variable neighborhood descent algorithm for flexible job shop scheduling problems, Computers and Operations Research, 35 (2008) 2892-2907.

[29] Vilcot G., Billaut J.C., A tabu search algorithm for solving a multi-criteria flexible job shop scheduling problem, International Journal of Production Research, 49 (2011) 6963-6980.

[30] Gao J., Gen M., Sun L.Y., Scheduling jobs and maintenances in flexible job shop with a hybrid genetic algorithm. Journal of Intelligent Manufacturing, 17 (2006) 493-507. 
[31] Perez M. A. F., Raupp F. M. P., A newton-based heuristic algorithm for multi-objective flexible job shop scheduling problem. Journal of Intelligent Manufacturing, DOI: 10.1007/s10845-014-0872-0.

[32] Mati Y., Rezg N., Xie X., An integrated greedy heuristic for flexible job shop scheduling problem. Proceeding of IEEE International Conference on Systems, Man and Cybernetics, 2001, 2534-2539.

[33] Wang S. J., Zhou B. H., Xi L. F., A filtered-beam-search-based heuristic algorithm for flexible job-shop scheduling problem. International Journal of Production Research, 46 (2008) 3027-3058.

[34] Wang S. J., Yu J.B., An effective heuristic for flexible job-shop scheduling problem with maintenance activities. Computer and Industrial Engineering, 59 (2010) 436-447.

[35] Ziaee M., A heuristic algorithm for solving flexible job shop scheduling problem. International Journal of Advanced Manufacturing Technology, 71 (2014) 519-528.

[36] Ziaee M., A heuristic algorithm for the distributed and flexible job-shop scheduling problem. Journal Supercomputing, 67 (2014) 69-83.

[37] Clleja G., Pastor R., A dispatching algorithm for flexible job-shop scheduling with transfer batches: an industrial application, 25 (2014) 93-109.

[38] Chen J.C., Chen K.H., Wu J.J., Che CW, A study of the flexible job shop scheduling problem with parallel machines and reentrant process. International Journal of Advanced Manufacturing Technology, 39 (2008) 344-354.

[39] Ben H. A, Haouari M., Huguet M.J., Lopez P., Discrepancy search for the flexible job shop scheduling problem. Computer \& Operations Research, 37 (2010) 2192-2201.

[40] Ozguven C., Yavuz Y., Ozbakir L, Mixed integer goal programming models for the flexi le job-shop scheduling problems with separable and non-separable sequence dependent setup times. Applied Mathematical Modelling, 36 (2012) 846-858.

[41] He W., Sun D.H., Scheduling flexible job shop problem subject to machine breakdown with route changing and right-shift strategies. International Journal of Advanced Manufacturing Technology, 66 (2013) 501-514.

[42] Sun D.H., He W., Zheng L.J., Liao X.Y., Scheduling flexible job shop problem subject to machine breakdown with game theory. International Journal of Production Research, 52 (2014) 3858-3876.

[43] Chan F.T.S., Wong T.C., Chan L.Y., Flexible job-shop scheduling problem under resource constraints. International o Journal of Production Research, 44 (2006) 2071-2089.

[44] Ho N.B., Tay J.C., Lai E.M.K., An effective architecture for learning and evolving flexible job-shop schedules. European Journal of Operational Research, 179 (2007) 316-333.

[45] De G. L., Pezzella F., An improved genetic algorithm for the distributed and flexible job-shop scheduling problem. European Journal of Operational Research, 200 (2010) 395-408.

[46] Mati Y., Lahlou C., Dauzere-Peres S., Modelling and solving a practical flexible job shop scheduling problem with blocking constraints. International Journal of Production Research, 49 (2011) 2169-2182.

[47] AI-Hinai N., EIMekkawy T.Y., Robust and stable flexible job shop scheduling with random machine breakdowns using a hybrid genetic algorithm. International Journal of Production Research, 132 (2011) 279291. 
[48] Chen J. C., Wu C. C., Chen C. W. et al., Flexible job shop scheduling with parallel machines using genetic algorithm and grouping genetic algorithm. Experts systems with applications, 39 (2012) 10016-10021.

[49] Gholami M., Zandieh M., Integrating simulation algorithm to schedule a dynamic flexible job shop. Journal of Intelligent Manufacturing, 20 (2009) 481-498.

[50] Rahmati S.H.A., Zandieh M., Yazdani M., Developing two multi-objective evolutionary algorithms for the multi-objective flexible job shop scheduling problem. International Journal of Advanced Manufacturing Technology, 64 (2013) 915-932.

[51] Chiang T.C., Lin H.J., A simple and effective evolutionary algorithm for multiobjective flexible job shop scheduling. International Journal of Production Economics, 141 (2013) 87-98.

[52] Demir Y., Isleyen S.K., An effective genetic algorithm for flexible job shop scheduling with overlapping in operations. International Journal of Production Research, 52 (2014) 3905-3921.

[53] Zhang G.H., Gao L., Shi Y., An effective genetic algorithm for the flexible job shop scheduling problem. Experts systems with applications, 38 (2011) 3563-3573.

[54] Wang X.J. Gao L., Zhang C.Y., Shao X.Y., A multi-objective genetic algorithm based on immune and entropy pimple for flexible job shop scheduling problem. International Journal of Advanced Manufacturing Techonlogy, 51 (2010) 757-767.

[55] Lei D.M., Co-evolutionary genetic algorithm for fuzzy flexible job shop scheduling. Applied Soft Computing, 12 (2012) 2237-2245.

[56] Defersha F.M., Chen M.Y., A parallel genetic algorithm for a flexible job-shop scheduling problem with sequence dependent setups. International Journal of Advanced Manufacturing Technology, 49 (2009) 263279.

[57] Rossi A, Dini G, Flexible job-shop scheduling with routing flexibility and separable setup times using ant colony optimization method. Robotics and Computer-integrated Manufacturing, 23 (2007) 503-516.

[58] Yao B.Z. Yang C.Y., Hu J.J, et al., An improved ant colony optimization for flexible job shop scheduling problems. Advanced Science Letters, 4 (2011) 2127-2131.

[59] Luo D.L., Chen H.P., Wu S.X., Shi Y.X., Hybrid ant colony multi-objective optimization for flexible job shop scheduling problems. Journal of Intelligent Manufacturing, 11 (2010) 361-369.

[60] Rossi A., Flexible job shop scheduling with sequence-dependent setup and transportation times by ant colony with reinforced pheromone relationships. International Journal of Production Economics, 153 (2014) 253-267.

[61] Huang R.H., Yang C.L., Cheng W.C., Flexible job shop scheduling with due window a two-pheromone ant colony approach. International Journal of Production Economics, 141 (2013) 685-697.

[62] Boukef H., Benrejeb M., Borne P., Flexible job-shop scheduling problems resolution inspired from particle swarm optimization. Studies in Informatics and Control, 17 (2008) 241-252.

[63] Liu H.B., Abraham A., Wang Z.W., A multi-swarm approach to multi-objective flexible job shop scheduling problems. Fundamental Informaticae, 95 (2009) 465-489. 
[64] Moslehi G., Mahnam M., A Pareto approach to multi-objective flexible job-shop scheduling problem using particle swarm optimization and local search. International Journal of Production Economic, 129 (2011) 1422.

[65] Nourali S., Imanipour N., A particle swarm optimization-based algorithm for flexible job shop scheduling problem with sequence dependent setup times. Scientia Iranica, 21 (2014) 1021-1033.

[66] Yuan Y., Xu H., Flexible job shop scheduling using hybrid differential evolution algorithms. Computes and Industrial Engineering, 65 (2013) 246-260.

[67] Xu X.L., Li L., Fan L.X. Zhang J., et al., Hybrid discrete differential evolution algorithm for lot splitting with capacity constraints in flexible job scheduling. Mathematical Problems in Engineering, 2013, article ID: 986218, 10pages.

[68] Fattahi P., Jolai F., Arkat J., Flexible job shop scheduling with overlapping in operations. Applied Mathematical Modelling, 33 (2009) 3076-3087.

[69] Logendran R., Sonthinen A., ATabu search-based approach for scheduling job-shop type flexible manufacturing systems. Journal of Operational Research Society, 48 (1997) 264-277.

[70] Saidi-Mehrabad M., Fattahi P., Flexible job shop scheduling with tabu search algorithms. International Journal of Advanced Manufacturing Technology, 32 (2007) 563-570.

[71] Li J.Q., Pan Q.K., Liang Y.C., An effective hybrid tabu search algorithm for multi-objective flexible jobshop scheduling problems. Computers \& Industrial Engineering, 59 (2010) 647-662.

[72] Li J.Q., Pan Q.K., Suganthan P.N., Chua T.J., A hybrid tabu search algorithm with an efficient neighborhood structure for the flexible job shop scheduling problem. International Journal of Advanced Manufacturing Technology, 52 (2001) 683-697.

[73] Jia S., Hu Z.H., Path-relinkinng tabu search for the multi-objective flexible job shop scheduling problem. Computers \& Operations Research, 47 (2014) 11-26.

[74] Eshlaghy A.T., Sheibatolhamdy S.A., Scheduling in flexible job-shop manufacturing systems by improved tabu search. African Journal of Business Management, 5 (2011) 4863-4872.

[75] Wang L., Wang S.Y., Xu Y., Zhou G., A bi-population based estimation of distribution algorithm for the flexible job-shop scheduling problem. Comupter \& Industrial Engineering, 62 (2012) 917-926.

[76] Wang L., Wang S.Y., Liu M., A Pareto-based estimation of distribution algorithm for the multi-objective flexible job-shop scheduling problem. International Journal of Production Research, 51 (2013) 3574-3592.

[77] Wang S.Y., Wang L., Xu Y., Liu M., An effective estimation of distribution algorithm for the flexible jobshop scheduling problem with fuzzy processing time. International Jouornal of Production Research, 51 (2013) 3778-3793.

[78] Amiri M., Zandieh M., Yazdani M., Bagheri A., A variable neighborhood search algorithm for the flexible job-shop scheduling problem. International Journal of Production Research, 48 (2010) 5671-5689.

[79] Yazdani M., Amiri M., Zandieh M., Flexible job-shop scheduling with parallel variable neighborhood search algorithm. Experts systems with Applications, 37 (2010) 678-687. 
[80] Bagheri A., Zandieh M., Bi-criterial flexible job-shop scheduling with sequence-dependent setup timesvariable neighborhood search approach. Journal of Manufacturing Systems, 30 (2011) 8-15.

[81] Karimi H., Rahmati S.H.A., Zandieh M., An effective knowledge-based algorithm for the flexible job shop scheduling problem. Knowledge-based Systems, 36 (2012) 236-244.

[82] Lei D.M., Guo X.P., Variable neighborhood search for dual-resource constrained flexible job shop scheduling. International Journal of Production Research, 52 (2014) 2519-2529.

[83] Tanev I.T., Uozumi T., Morotome Y., Hybrid evolutionary algorithm-based real-wrold flexible job shop scheduling problem: application service provider approach. Applied Soft Computing, 5 (2004) 87-100.

[84] Fattahi P., Mehrabad M.S., Jolai F., Mathematical modeling and heuristic approaches to flexible job shop scheduling problems. Journal of Intelligent Manufacturing, 18 (2007) 331-342.

[85] Frutos M., Olivera A.C., Tohme F., A memetic algorithm based on NSGAII scheme for the flexible jobshop scheduling. Annals of Operations Research, 181 (2010) 745-765.

[86] Roshanaei V., Azab A., EIMaraghy H., Mathematical modeling and a meta-heuristics for flexible job shop scheduling. International Journal of Production Research, 51 (2013) 6247-6274.

[87] Shahsavari-Pour N., Ghasemishabankareh B., A novel hybrid meta-heuristic algorithm for solving multi objective flexible job shop scheduling. Journal of Manufacturing Systems, 32 (2013) 771-780.

[88] Gao J., Gen M., Sun L.Y., Zhao X.H., A hybrid genetic algorithm and bottleneck shifting for multiobjective flexible job shop scheduling problems. Computer and Industrial Engineering, 53 (2007) 149-162.

[89] Xing L.N., Chen Y.W., Yang K.W., Multi-population interactive co-evolutionary algorithm for flexible job shop scheduling problems. Computation Optimization and Applications, 48 (2011) 139-155.

[90] Zhang Q., Manier H., Manier M.A., A genetic algorithm with tabu search procedure for flexible job shop scheduling with transportation constraints and bounded processing time. Computer and Operations Research, 39 (2012) 1713-1723.

[91] Barzegar B., Motameni H., Bozorgi H., Solving flexible job-shop scheduling problem using gravitational search algorithm and colored petri-net. Journal of Applied Mathematics, 2012, Article ID: 651310, 20 pages.

[92] Liouane N., Saad I., Hammadi S., Borne P., Ant system \& local search optimization for flexible job shop scheduling production. International Journal of Computers Communications \& Control, 2 (2007) 174-184.

[93] Xia W.J., Wu Z.M., An effective hybrid optimization approach for multi-objective flexible job shop scheduling problems. Computers and Industrial Engineering, 48 (2005) 409-425.

[94] Shao X.Y., Hybrid discrete particle swarm optimization for multi-objective flexible job shop scheduling problem. International Journal of Advanced Manufacturing Technology, 67 (2013) 2885-2901.

[95] Araghi M.E.T., Jolai F., Rabiee M., Incorporating learning effect and deterioration for solving a SDST flexible job-shop scheduling problem with a hybrid meta-heuristic approach. International Journal of Computer Integrated Manufacturing, 27 (2014) 733-746.

[96] Sadrzadeh A., Development of both the AIS and PSO for solving the flexible job shop scheduling problem. Arabian Journal for Science and Engineering, 38 (2013) 3593-3604. 
[97] Dalfard V.M., Mohammadi G., Two meta-heuristic algorithms for solving multi-objective flexible job shop scheduling with parallel machine and maintenance constraints. Computers \& Mathematics with Application, 64 (2012) 2111-2117.

[98]Wu Z.B., Weng M.X., Multi-agent scheduling method with earliness and tardiness objectives in flexible job shops, IEEE Transactions on System, Man, and Cybernetics-Part B: Cybernetics, 35 (2005) 293-301.

[99] Yu X.F., Ram B., Jiang X.C., Parameter setting in a bio-inspired model for dynamic flexible job shop scheduling with sequence-dependent setups. European Journal of Industrial Engineering, 1 (2007) 182-199.

[100] Rajabinasab A, Mansour S, Dynamic flexible job shop scheduling with alternative process plans: an agentbased approach. International Journal of Advanced Manufacturing Technology, 54 (2011) 1091-1107.

[101] Moradi E., Ghomi S., Zandieh M., An effective architecture for scheduling flexible job-shop with machine availability constraints. International Journal of Advanced Manufacturing Technology, 51 (2010) 325-339.

[102] Bagheri A., Zandieh M., Mahdavi I., An artificial immune algorithm for the flexible job-shop scheduling problem. Future Generation Computers Systems-the International Journal of Grid Computing and Escience, 26 (2010) 533-541.

[103] Karthikeyan S., Asokan P., Nickolas S., Ahybrid discrete firefly algorithm for multi-objective flexible job shop scheduling problem with limited resource constraints. International Journal of Advanced Manufacturing Technology, 72 (2014) 1567-1579.

[104] Li J.Q., Pan Q.K., Xie S.X., An effective shuffled frog-leaping algorithm for multi-objective flexible job shop scheduling problems. Applied Mathematics and Computation, 218 (2012) 9353-9371.

[105] Li J.Q. Pan Q.K., Chemical-reaction optimization for flexible job-shop scheduling problems with maintenance activity. Applied Soft Computing, 12 (2012) 2896-2912.

[106] Rajkumar M., Asokan P., Vamsikrishn V., A GRASP algorithm for flexible job-shop scheduling with maintenance constraints. International Journal of Production Research, 48 (2010) 6821-6836.

[107] Ra jkumar M., Asokan P., Page T., A GRASP algorithms for flexible job-shop scheduling problem with limited resource constraints. International Journal of Production Research, 49 (2010) 2409-2423.

[108] Farughi H., Yegane B.Y., Fathian M., A new critical path method and a memetic algorithm for flexible job shop scheduling with overlapping operations. Simulation-Transactions of the Society for Modeling and Simulation International, 89 (2013) 264-277.

[109] Nie L., Gao L., Li P.G., Li X.Y., A GEP-based reactive scheduling policies constructing approach for dynamic flexible job shop scheduling problem with job release dates. Journal of Intelligent Manufacturing, 24 (2013) 763-744.

[110] Rahmati S.H.A., Zandieh M., A new biogeography-based optimization (BBO) algorithm for the flexible job shop scheduling problem. International Journal of Advanced Manufacturing Technology, 58 (2012) 1115-1129.

[111] Geem Z.W., Kim J.H., Loganathan G.V., A new heuristic optimization algorithm: harmony search, Simulation, 76 (2001) 60-68. 
[ 112 ]Mahdavi M., Fesanghary M., Damangir E., An improved harmony search algorithm for solving optimization problems, Applied Mathematics and Computation, 188 (2007) 1567-1579.

[113]Lee K.S., Geem Z.W., Lee S.H., Bae K.W., The harmony search heuristic algorithm for discrete structural optimization, Engineering Optimization, 37 (2005) 663-684.

[114] Omran M.G.H., Mahdavi M., Global-best harmony search, Applied Mathematics and Computation, 198 (2008) 643-656.

[115]Das S., Mukhopadhyay A., Roy A., Abraham A., Panigrahi B.K.R., Exploratory power of the harmony search algorithm: analysis and improvements for global numerical optimization, IEEE Transactions on System, Man, and Cybernetics, Part B: Cybernetics, 41 (2011) 89-106.

[116]Geem Z.W., Optimal scheduling of multiple dam system using harmony search algorithm, Lecture Notes in Computer Science, 4507 (2007) 316-323.

[117]Wang L., Pan Q.K., Tasgetiren M.F., Minimizing the total flow time in a flow shop with blocking by using hybrid harmony search algorithms, Expert System with Applications, 37 (2010) 7929-7936.

[118]Pan Q.K., Suganthan P.N., Liang J.J., Tasgetiren M.F., A local-best harmony search algorithm with dynamic sub-harmony memories for lot-streaming flow shop scheduling problem, Expert System with Applications, 38 (2011) 3252-3259.

[119]Gao K.Z., Pan Q.K., Li J.Q., Liang J.J., A hybrid harmony search algorithm for the no-wait flow shop scheduling problems, Asia-Pacific J of Operational research, 29 (2012) 1250012 1-23.

[120]Gao K.Z., Pan Q.K., Li J.Q., Discrete harmony search algorithm for the no-wait flow shop scheduling problem with total flow time criterion, International Journal of Advanced Manufacturing Technology, 56 (2011) 683-692.

[121] Yuan Y., Xu H., Yang J.D., A hybrid harmony search algorithm for the flexible job shop scheduling problem. Applied Soft Computing, 13 (2013) 3259-3272.

[122] Yuan Y., Xu H., An integrated search heuristic for large-scale flexible job shop scheduling problems. Computer \& Operations Research, 40 (2013) 2864-2877.

[123]Gao K.Z., Suganthan P.N., Pan Q.K., Chua T.J., Cai T.X., Chong C.S., Pareto-based grouping discrete harmony search algorithm for multi-objective flexible job shop scheduling, Information Sciences, 289 (2014) 76-90.

[124] Karaboga, D., An idea based on honey bee swarm for numerical optimization, Technical Report TR06. Turkey: Computer Engineering Department, Erciyes University.

[125] Karaboga, N., A new design method based on artificial bee colony algorithm for digital IIR filters. Journal of the Franklin Institute 346 (2009) 328-348.

[126] Karaboga, D., and B. Akay, A comparative study of artificial bee colony algorithm. Applied Mathematics and Computation, 24 (2009) 108-132.

[127] Karaboga, D., and B. Basturk, A powerful and efficient algorithm for numerical function optimization: artificial bee colony (ABC) algorithm. Journal of Global Optimization, 39 (2007) 459-471. 
[128] Karaboga, D., and B. Basturk, On the performance of artificial bee colony (ABC) algorithm. Applied Soft Computing, 8 (2008) 687-697.

[129] Banharnsakun, A., T. Achalakul, and B. Sirinaovakul, The best-so-far selection in artificial bee colony algorithm. Applied Soft Computing, 11 (2011) 2888-2901.

[130] Wong, L. P., Low M. Y. H., and Chong C. S., Bee colony optimization with local search for traveling salesman problem. Proceedings of 6th IEEE International Conference on Industrial Informatics: 2008, 10191025 .

[131] Pan, Q. K., Tasgetiren M. F., Suganthan P. N., and Chua T. J., A discrete artificial bee colony algorithm for the lot-streaming flow shop scheduling problem. Information Sciences, 181 (2010) 2455-2468.

[132] Banharnsakun, A., B. Sirinaovakul, and T. Achalakul. "Job shop scheduling with the best-so-far ABC." Engineering Applications of Artificial Intelligence, 25 (2012) 583-593.

[133] Li J.Q., Pan Q.K., Xie S.X., Wang S., A hybrid artificial bee colony algorithm for flexible job shop scheduling problems. International Journal of Compuers Communications and Control, 6 (2011) 286-296.

[134] Li J.Q., Pan Q.K., Tasgetiren M.F., A discrete artificial bee colony algorithm for the multi-objective flexible job shop scheduling with maintenance activities. Applied Mathematical Modelling, 38 (2014) 11111132 .

[135] Wang, L., G. Zhou, Y. Xu, S. Y. Wang, and M. Liu. An effective artificial bee colony algorithm for the flexible job-shop scheduling problem. International Journal of Advanced Manufacturing Technology 60 (2012a) 303-315.

[136] Wang, L., G. Zhou, Y. Xu, and M. Liu. "An enhanced Pareto-based artificial bee colony algorithm for the multi-objective flexible job-shop scheduling." International Journal of Advanced Manufacturing Technology, 60 (2012b) 1111-1123.

[137] Wang L., Zhou G., Xu Y., Liu M., A hybrid artificial bee colony algorithm for the fuzzy flexible job shop scheduling problem. International Journal of Production Research, 51 (2013) 3593-3608.

[138]Liang J.J., Suganthan P.N., Dynamic multi-swarm particle swarm optimizer, Proc. 2005 IEEE Conf. swarm intelligence symposium, SIS'05, Pasadena, California, USA, 2005, pp. 124-129.

[139]Zhang C.Y., Li P.G., Guan Z.L., Rao Y.Q., A tabu search algorithm with a new neighborhood structure for the job shop scheduling problem, Computers and Operations Research, 34 (2007) 3229-3242.

[140] Kacem I., Hammadi S., Borne P., Pareto-optimality approach for flexible job-shop scheduling problems: hybridization of evolutionary algorithms and fuzzy logic, Mathematics and Computers in Simulation, 60 (2002) 245-276.

[141]Brandimarte P., Routing and scheduling in a flexible job shop by tabu search, Annals of Operations Research, 41 (1993) 157-183.

[142] Xing, L. N., Chen, Y. W., \& Yang, K. W., An efficient search method for multi-objective flexible job shop scheduling problems. Journal of Intelligent Manufacturing, 20 (2009) 283-293. 
[143] Seyed Habib A. Rahmati, Proposing a Pareto-based Multi-Objective Evolutionary Algorithm to Flexible Job Shop Scheduling Problem, World Academy of Science, Engineering and Technology, 61 (2012) 11601165.

[144]Rabiee M., Zandieh M., Ramezani P., Bi-objective partial flexible job shop scheduling problems: NSGAII, NRGA, MOGA and PAES approaches, International Journal of Production Research, 50 (2012)73277342.

[145]Zhao S.Z., Suganthan P. N., Zhang Q., Decomposition-based multi-objective evolutionary algorithm with an ensemble of neighborhood sizes, IEEE Transactions on Evolutionary Computation, 16 (2012) 442-446.

[146]Wang X., Gao L., Zhang G., Shao X., A multi-objective genetic algorithm based on immune and entropy principle for flexible job-shop scheduling problem, International Journal of Advanced Manufacturing Technology, 51 (2010) 757-767.

[147] Lei D.M., A Genetic algorithm for flexible job shop scheduling with fuzzy processing time, International Journal of Production Research, 48 (2010) 2995-3013. 


\section{Appendix}

\section{A. The benchmark instances used in this thesis}

In the first line there are (at least) 2 numbers: the first is the number of jobs and the second the number of machines (the 3 rd is not necessary; it is the average number of machines per operation)

Every row represents one job: the first number is the number of operations of that job, the second number (let's say $\mathrm{k}>=1$ ) is the number of machines that can process the first operation; then according to $\mathrm{k}$, there are $\mathrm{k}$ pairs of numbers (machine, processing time) that specify which are the machines and the processing times; then the data for the second operation and so on...

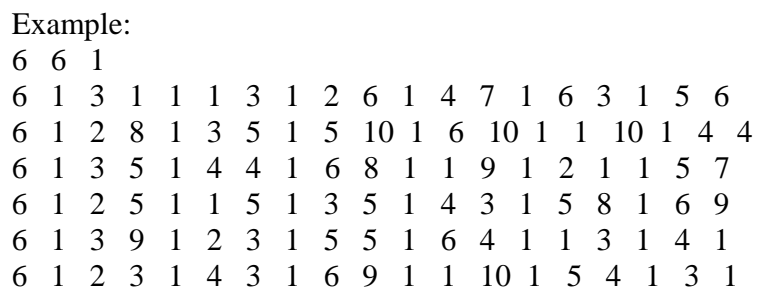

\section{Kacem01:}

$4,5,0$,

$3,5,1,2,2,5,3,4,4,1,5,2,5,1,5,2,4,3,5,4,7,5,5,5,1,4,2,5,3,5,4,4,5,5$, $3,5,1,2,2,5,3,4,4,7,5,8,5,1,5,2,6,3,9,4,8,5,5,5,1,4,2,5,3,4,4,54,5,5$, $3,5,1,9,2,8,3,6,4,7,5,9,5,1,6,2,1,3,2,4,5,5,4,5,1,4,2,5,3,2,4,1,5,5$, $2,5,1,1,2,5,3,2,4,4,5,12,5,1,5,2,1,3,2,4,1,5,2$

\section{Macem02:}

$8,8,0$,

$3,7,1,5,2,3,3,5,4,3,5,3,7,10,8,9,7,1,10,3,5,4,8,5,3,6,9,7,9,8,6,6,2,10,4,5,5,6,6,2,7,4,8,5$,

$4,6,1,5,2,7,3,3,4,9,5,8,7,9,7,2,8,3,5,4,2,5,6,6,7,7,10,8,9,6,2,10,4,5,5,6,6,4,7,1,8,7,6,1,10,2,8,3,9,4,6,5,4,6,7$, $3,6,1,10,4,7,5,6,6,5,7,2,8,4,6,2,10,3,6,4,4,5,8,6,9,7,10,6,1,1,2,4,3,5,4,6,6,10,8,4$, $3,8,1,3,2,1,3,6,4,5,5,9,6,7,7,8,8,4,8,1,12,2,11,3,7,4,8,5,10,6,5,7,6,8,9,8,1,4,2,6,3,2,4,10,5,3,6,9,7,5,8,7$, $4,6,1,3,2,6,3,7,4,8,5,9,7,10,6,1,10,3,7,4,4,5,9,6,8,7,6,6,2,9,3,8,4,7,5,4,6,2,7,7,7,1,11,2,9,4,6,5,7,6,5,7,3,8,6$, $3,7,1,6,2,7,3,1,4,4,5,6,6,9,8,10,7,1,11,3,9,4,9,5,9,6,7,7,6,8,4,6,1,10,2,5,3,9,4,10,5,11,7,10$, $3,6,1,5,2,4,3,2,4,6,5,7,7,10,6,2,9,4,9,5,11,6,9,7,10,8,5,6,2,8,3,9,4,3,5,8,6,6,8,10$, $4,6,1,2,2,8,3,5,4,9,6,4,8,10,6,1,7,2,4,3,7,4,8,5,9,7,10,7,1,9,2,9,4,8,5,5,6,6,7,7,8,1,6,1,9,3,3,4,7,5,1,6,5,7,8$

\section{Kacem03:}

$10,7,0$,

$3,7,1,1,2,4,3,6,4,9,5,3,6,5,7,2,7,1,8,2,9,3,5,4,4,5,1,6,1,7,3,7,1,4,2,8,3,10,4,4,5,11,6,4,7,3$, $2,7,1,6,2,9,3,8,4,6,5,5,6,10,7,3,7,1,2,2,10,3,4,4,5,5,9,6,8,7,4$, $3,7,1,15,2,4,3,8,4,4,5,8,6,7,7,1,7,1,9,2,6,3,1,4,10,5,7,6,1,7,6,7,1,11,2,2,3,7,4,5,5,2,6,3,7,14$, $3,7,1,2,2,8,3,5,4,8,5,9,6,4,7,3,7,1,5,2,3,3,8,4,1,5,9,6,3,7,6,7,1,1,2,2,3,6,4,4,5,1,6,7,7,2$, $3,7,1,7,2,1,3,8,4,5,5,4,6,3,7,9,7,1,2,2,4,3,5,4,10,5,6,6,4,7,9,7,1,5,2,1,3,7,4,1,5,6,6,6,7,2$, $3,7,1,8,2,7,3,4,4,56,5,9,6,8,7,4,7,1,5,2,14,3,1,4,9,5,6,6,5,7,8,7,1,3,2,5,3,2,4,5,5,4,6,5,7,7$, $3,7,1,5,2,6,3,3,4,6,5,5,6,15,7,2,7,1,6,2,5,3,4,4,9,5,5,6,4,7,3,7,1,9,2,8,3,2,4,8,5,6,6,1,7,7$, $3,7,1,6,2,1,3,4,4,1,5,10,6,4,7,3,7,1,11,2,13,3,9,4,8,5,9,6,10,7,8,7,1,4,2,2,3,7,4,8,5,3,6,10,7,7$, $3,7,1,12,2,5,3,4,4,5,5,4,6,5,7,5,7,1,4,2,2,3,15,4,99,5,4,6,7,7,3,7,1,9,2,5,3,11,4,2,5,5,6,4,7,2$, $3,7,1,9,2,4,3,13,4,10,5,7,6,6,7,8,7,1,4,2,3,3,25,4,3,5,8,6,1,7,2,7,1,1,2,2,3,6,4,11,5,13,6,3,7,5$ 


\section{Kacem04:}

$10,10,0$,

, 10,1,1,2,4,3,6,4,9,5,3,6,5,7,2,8,8,9,9,10,5,10,1,4,2,1,3,1,4,3,5,4,6,8,7,10,8,4,9,11,10,4,10,1,3,2,2,3,5,4,1,5,5,6,6,7,9,8,5,9, $10,10,3$,

,10,1,2,2,10,3,4,4,5,5,9,6,8,7,4,8,15,9,8,10,4,10,1,4,2,8,3,7,4,1,5,9,6,6,7,1,8,10,9,7,10,1,10,1,6,2,11,3,2,4,7,5,5,6,3,7,5,8,1 $4,9,9,10,2$,

, $10,1,8,2,5,3,8,4,9,5,4,6,3,7,5,8,3,9,8,10,1,10,1,9,2,3,3,6,4,1,5,2,6,6,7,4,8,1,9,7,10,2,10,1,7,2,1,3,8,4,5,5,4,6,9,7,1,8,2,9,3,1$

0,4 ,

, $10,1,5,2,10,3,6,4,4,5,9,6,5,7,1,8,7,9,1,10,6,10,1,4,2,2,3,3,4,8,5,7,6,4,7,6,8,9,9,8,10,4,10,1,7,2,3,3,12,4,1,5,6,6,5,7,8,8,3,9$, $5,10,2$,

,10,1,7,2,10,3,4,4,5,5,6,6,3,7,5,8,15,9,2,10,6,10,1,5,2,6,3,3,4,9,5,8,6,2,7,8,8,6,9,1,10,7,10,1,6,2,1,3,4,4,1,5,10,6,4,7,3,8,11, $9,13,10,9$,

,10,1,8,2,9,3,10,4,8,5,4,6,2,7,7,8,8,9,3,10,10,10,1,7,2,3,3,12,4,5,5,4,6,3,7,6,8,9,9,2,10,15,10,1,4,2,7,3,3,4,6,5,3,6,4,7,1,8,5, $9,1,10,11$

,10,1,1,2,7,3,8,4,3,5,4,6,9,7,4,8,13,9,10,10,7,10,1,3,2,8,3,1,4,2,5,3,6,6,7,11,8,2,9,13,10,3,10,1,5,2,4,3,2,4,1,5,2,6,1,7,8,8,1 $4,9,5,10,7$,

, 10,1,5,2,7,3,11,4,3,5,2,6,9,7,8,8,5,9,12,10,8,10,1,8,2,3,3,10,4,7,5,5,6,13,7,4,8,6,9,8,10,4,10,1,6,2,2,3,13,4,5,5,4,6,3,7,5,8, $7,9,9,10,5$

,10,1,3,2,9,3,1,4,3,5,8,6,1,7,6,8,7,9,5,10,4,10,1,4,2,6,3,2,4,5,5,7,6,3,7,1,8,9,9,6,10,7,10,1,8,2,5,3,4,4,8,5,6,6,1,7,2,8,3,9,10, 10,12 ,

, $10,1,4,2,3,3,1,4,6,5,7,6,1,7,2,8,6,9,20,10,6,10,1,3,2,1,3,8,4,1,5,9,6,4,7,1,8,4,9,17,10,15,10,1,9,2,2,3,4,4,2,5,3,6,5,7,2,8,4,9$ $, 10,10,23$

\section{Kacem05:}

$15,10,0$,

$4,10,1,1,2,4,3,6,4,9,5,3,6,5,7,2,8,8,9,9,10,4,10,1,1,2,1,3,3,4,4,5,8,6,10,7,4,8,11,9,4,10,3,10,1,2,2,5,3,1,4,5,5,6,6,9,7,5,8,10$, $9,3,10,2,10,1,10,2,4,3,5,4,9,5,8,6,4,7,15,8,8,9,4,10,4$,

$4,10,1,4,2,8,3,7,4,1,5,9,6,6,7,1,8,10,9,7,10,1,10,1,6,2,11,3,2,4,7,5,5,6,3,7,5,8,14,9,9,10,2,10,1,8,2,5,3,8,4,9,5,4,6,3,7,5,8,3$, $9,8,10,1,10,1,9,2,3,3,6,4,1,5,2,6,6,7,4,8,1,9,7,10,2$

$4,10,1,7,2,1,3,8,4,5,5,4,6,9,7,1,8,2,9,3,10,4,10,1,5,2,10,3,6,4,4,5,9,6,5,7,1,8,7,9,1,10,6,10,1,4,2,2,3,3,4,8,5,7,6,4,7,6,8,9,9,8$ $, 10,4,10,1,7,2,3,3,12,4,1,5,6,6,5,7,8,8,3,9,5,10,2$,

$4,10,1,6,2,2,3,5,4,4,5,1,6,2,7,3,8,6,9,5,10,4,10,1,8,2,5,3,7,4,4,5,1,6,2,7,36,8,5,9,8,10,5,10,1,9,2,6,3,2,4,4,5,5,6,1,7,3,8,6,9,5$ $, 10,2,10,1,11,2,4,3,5,4,6,5,2,6,7,7,5,8,4,9,2,10,1$,

$4,10,1,6,2,9,3,2,4,3,5,5,6,8,7,7,8,4,9,1,10,2,10,1,5,2,4,3,6,4,3,5,5,6,2,7,28,8,7,9,4,10,5,10,1,6,2,2,3,4,4,3,5,6,6,5,7,2,8,4,9,7$ $10,9,10,1,6,2,5,3,4,4,2,5,3,6,2,7,5,8,4,9,7,10,2$,

$2,10,1,4,2,1,3,3,4,2,5,6,6,9,7,8,8,5,9,4,10,2,10,1,1,2,3,3,6,4,5,5,4,6,7,7,5,8,4,9,6,10,5$,

$2,10,1,1,2,4,3,2,4,5,5,3,6,6,7,9,8,8,9,5,10,4,10,1,2,2,1,3,4,4,5,5,2,6,3,7,5,8,4,9,2,10,5$,

$4,10,1,2,2,3,3,6,4,2,5,5,6,4,7,1,8,5,9,8,10,7,10,1,4,2,5,3,6,4,2,5,3,6,5,7,4,8,1,9,2,10,5,10,1,3,2,5,3,4,4,2,5,5,6,49,7,8,8,5,9,4$ ,10,5,10,1,1,2,2,3,36,4,5,5,2,6,3,7,6,8,4,9,11,10,2,

$4,10,1,6,2,3,3,2,4,22,5,44,6,11,7,10,8,23,9,5,10,1,10,1,2,2,3,3,2,4,12,5,15,6,10,7,12,8,14,9,18,10,16,10,1,20,2,17,3,12,4,5$, $5,9,6,6,7,4,8,7,9,5,10,6,10,1,9,2,8,3,7,4,4,5,5,6,8,7,7,8,6,9,56,10,2$,

$4,10,1,5,2,8,3,7,4,4,5,56,6,3,7,2,8,5,9,4,10,1,10,1,2,2,5,3,6,4,9,5,8,6,5,7,4,8,2,9,5,10,4,10,1,6,2,3,3,2,4,5,5,4,6,7,7,4,8,5,9,2$ $10,1,10,1,3,2,2,3,5,4,6,5,5,6,8,7,7,8,4,9,5,10,2$

$4,10,1,1,2,2,3,3,4,6,5,5,6,2,7,1,8,4,9,2,10,1,10,1,2,2,3,3,6,4,3,5,2,6,1,7,4,8,10,9,12,10,1,10,1,3,2,6,3,2,4,5,5,8,6,4,7,6,8,3,9$, $2,10,5,10,1,4,2,1,3,45,4,6,5,2,6,4,7,1,8,25,9,2,10,4$

$4,10,1,9,2,8,3,5,4,6,5,3,6,6,7,5,8,2,9,4,10,2,10,1,5,2,8,3,9,4,5,5,4,6,75,7,63,8,6,9,5,10,21,10,1,12,2,5,3,4,4,6,5,3,6,2,7,5,8,4$ ,9,2,10,5,10,1,8,2,7,3,9,4,5,5,6,6,3,7,2,8,5,9,8,10,4 ,

$4,10,1,4,2,2,3,5,4,6,5,8,6,5,7,6,8,4,9,6,10,2,10,1,3,2,5,3,4,4,7,5,5,6,8,7,6,8,6,9,3,10,2,10,1,5,2,4,3,5,4,8,5,5,6,4,7,6,8,5,9,4$, $10,2,10,1,3,2,2,3,5,4,6,5,5,6,4,7,8,8,5,9,6,10,4$

$4,10,1,2,2,3,3,5,4,4,5,6,6,5,7,4,8,85,9,4,10,5,10,1,6,2,2,3,4,4,5,5,8,6,6,7,5,8,4,9,2,10,6,10,1,3,2,25,3,4,4,8,5,5,6,6,7,3,8,2,9$, $5,10,4,10,1,8,2,5,3,6,4,4,5,2,6,3,7,6,8,8,9,5,10,4$

$4,10,1,2,2,5,3,6,4,8,5,5,6,6,7,3,8,2,9,5,10,4,10,1,5,2,6,3,2,4,5,5,4,6,2,7,5,8,3,9,2,10,5,10,1,4,2,5,3,2,4,3,5,5,6,2,7,8,8,4,9,7$, $10,5,10,1,6,2,2,3,11,4,14,5,2,6,3,7,6,8,5,9,4,10,8$,

\section{MK01:}

$10 \quad 6 \quad 2$

62153435335212346236526111313663643

5126131112226463652611 
5126234623652611334266621155

53652611126131353352123462

63533521365261112621534226463342666

6234621123342666126365261121342

51612134233426663265116131

5234623342666365261112622646

6161211553663643112334266622646

623462334266635335211612264621342

\section{MK02:}

$10 \quad 6 \quad 4$

66334513662253265346115633432665126263563322154

656156132442263561522243333221546334513662253

66115633432665653462466361233221545351423635264115245533635631446 3653

653514236352561561324421266115633432665514452363546411524553363

6653462466361251445236354143563144636535615613244222433

6563144636532653453514236352653462466361212656156132442

564115245533631526534624663612633451366225356314463653

622433535142363526534624663612563144636535144523635456156132442

512626534561561324425144523635422433

614365346246636125631446365364115245533632635656156132442

\section{MK03:}

$15 \quad 8 \quad 3$

1047158114551923184548187361131645721723192566334552818152111755102101 1285314371562819

10481873611316111722141355102101128531454111921861831326157134715811455 19457217231944111761383371562819

102335545721723192318452566344111761383371562819541119218618313345528 181117221413

10231845233555411192186183134411176138326157134572172319152481873611316 111725663

1026157133715628191524715811455195411192186183134572172319345528182566 323355551021011285314

102214132615713231845481873611316541119218618313551021011285314441117613 834715811455192566323355

10551021011285314441117613832214131117261571345721723191525411192186183 13231845371562819

103715628191117471581145519261571355102101128531444111761383541119218618 31322141323184523355

10111755102101128531448187361131637156281926157134411176138315222141354 1119218618313471581145519

10111726157133455281854111921861831344111761383231845256633715628194818 73611316551021011285314

1022141337156281948187361131623184525663111723355345528185510210112853 14541119218618313

1044111761383345528184818736113161117541119218618313371562819152233554 71581145519221413

10551021011285314152231845457217231926157134818736113164715811455195411 192186183132566344111761383

104818736113163455281822141345721723192566323184526157131525411192186 183131117

105510210112853142566326157134715811455194818736113161117541119218618313 345528182318454572172319

\section{MK04:}

$15 \quad 8 \quad 2$

8116216792673124275318398932348322556726147 
716126147116267313234832162172

616132348323327144242752173724431

5172116216792673124557

71722167924431318398921737323483224557

9162244313327144261472455731839892173716121679

525567217372614716226731

62455725567323483216216121679

9116216792443131839892427526147172217373234832

5255671161722455721679

43183989116323483224275

624275161116217373183989172

4162267312614725567

32556716124275

624557172318398932348323327144116

\section{MK05:}

$\begin{array}{lllll}15 & 4 & 2\end{array}$

62352721848216251372452624515

5137216251462452621826

824739235272451521848216251462182624936

72451524739218481482182624526146

62371524627247391382352721826

9146245261382371524627148218262184824515

5138247392162524627137

82371513824739245151371482493621625

9235271482452621625146218492184821826137

9218262184821849249362162513813714624526

721826218482162513714613824936

6148137247392162513821848

7148249362184824627246272182623715

7216252371521848218262451524627146

71382184924936137245262182621625

\section{MK06:}

\section{$\begin{array}{llll}10 & 15 & 3\end{array}$}

1542863729529712574149127104211823753858513882538109356116252519915 7462106122795624872521584218373102894537537933945811 1551388253810957414912710435611625215842183724872210612310289452795 6375379337538583945811297122118242863729552519915746 1521182279562106122487252158421837394581129712375379357414912710442 8637295513882538109310289455251991574635611623753858

1535611625251991574651388253810952158421837248722106123753858297123 7537933945811428637295211825741491271042795631028945

153102894521182394581129712375385852158421837356116237537934286372 95210612574149127104279565251991574651388253810924872

153753858513882538109279563561162525199157462487229712521584218375 74149127104428637295211823753793210612394581131028945

1535611623102894537538585138825381092118229712521584218373753793574 1491271043945811210612428637295279562487252519915746 1557414912710437537933753858211823561162525199157463102894539458112 $97124286372955138825381092487221061252158421837 \quad 27956$

1542863729539458113753858574149127104521584218372487229712310289455 1388253810921061252519915746375379327956211823561162

1521182428637295310289453753858375379321061227956394581157414912710 4525199157465138825381093561162521584218372487229712

\section{MK07:}

$\begin{array}{llll}20 & 5 & 3\end{array}$

52241152318115124141853852451727 
521351353852451727224115318512531351332

5521851419193314182411391243512314419

52241154410310217584518313221554105151239216231516

531351332231811552185141919333512314419145

5538524517272318115211557227117224115

51452115572241153135133244621731557

5446217315573318124154214414319515124227117

5521851419193344621731557318512542144143195152117515

5211557441031021758231516145531651741021017

514183185125538524517272115572117515

5351231441944103102175823151653852451727531651741021017

5211751514184217519453123318124153185125

525135331812415441031021758231811553852451727

5538524517272513535123144195316517410210172117515

554105151239216241139124211557145

55385245172742144143195153318124152315165218514191933

51243185125251352318115211557

53135133244621731557451831322151418213513

5145224115141821155754105151239216

\section{MK08:}

$20 \quad 10 \quad 2$

10271845257771319171424510121110110182710819231189235812

1212527184523581211101101923154191714159251495111927108191116

14251495111911101319271845245101223581211010159117271081911101101911018

1011010257771714111011018231571321014572311891911159

1215925149527184523118911101911117171424510122315419181811019

1023157131319159110192358122718452814109245101211018117

12111011018117159281410927108192315419210145718181101911191110

11111017141110231541925149527184513191119245101215911019

14271081928141091119110192101457125245101225777111611719111319111011018

111101921014571818231189117111025149523154191101813191119

11251495111018182315419271081923581223118928141091101019111319

10110192311892577711161714271845245101211101818251495

112101457110192710819231541911191818281410923118911018251495125

11111025777111019111714231571328141091116235812251495125

11251495257771714110102710819231541927184511723118911191818

111252710819110101911181821014572514951110111923157132814109

1311010251495159110191110235812125210145711102814109231571311161714

112315713125110191319181811715917142718451110251495

1027108191252311891911245101211018271845281410923581211019

10110181101017141911231571312528141092358121591116

\section{MK09:}

$\begin{array}{lll}20 & 10 \quad 3\end{array}$

12221011118171814111022161018296212479411310116251917191114161255799946 814616

13181725641122101112598822163114185141015612461081575282519174794113101 1611104116311717471416431158711917

11461081575282598822161018221011157999468146161416251917111025641122163 111314

114185141015612251917441181691516181414161817411631171747410681355281314 4794113101161110

14181714161594106813552841163117174722161018461081575281814256411425713 10105115799946814616259884185141015612251917

11425713101051122161018111013141595799946814616181718141254610815752844 1181691516

14181418172598814161110425713101051112525641157999468146164411816915165 2811981361410184610815752841163117174722161018 
1311104106813552815947941131011619114257131010511461081575281255281198 13614101857999468146162210111411631171747256411

11181712511101416256411479411310116528119813614101819112962122210111259 88

12141644118169151613144257131010511191157999468146162564114116311717472 210111221631141851410156121110

10191115952811981361410181416441181691516259884794113101161314111041163 1171747

1141068135528441181691516141629621246108157528479411310116125181457999 4681461625641122161018

11125131429621215942571310105114116311717472210111181725191711104794113 10116

104311587119171110221610182210111461081575284411816915161416411631171747 4794113101162216311

121110441181691516425713101051152811981361410182564112962121254106813552 814162216311221011146108157528

14181744118169151613142962121814461081575284794113101164257131010511418 51410156122210111141643115871191725191741068135528

13528119813614101819114794113101161817410681355282564111110461081575282 210111221610184116311717471314251917

115281198136141018579994681461625641141068135528131443115871191719112210 111425713101051118144185141015612

131314221011147941131011622161018221631144118169151641163117174742571310 10511410681355282598812546108157528159

13411631171747425713101051146108157528131425641144118169151615911101817 2962125281198136141018221631122161018

\section{MK10:}

$\begin{array}{lll}20 & 15 \quad 3\end{array}$

1226525271161112548103184109727917418714912473413882653811991310192165 21610931241151529101053752847416613511107

132711611421610959816265252211192312715441110145107154381125513115381199 13101921634138826481031841097411651110173629101052511211

114381125513112211192791726525416613511107291010553811991310192164810318 410974216109598162312715225919

11441110145107155381199131019216151512529101052711611411651110173621013611 22591934138826481031841097

142711611291010545117810112162101361141165111017362791743811255131112542 1610959816311521999416613511107221119441110145107155381199131019216

11311521999279174810318410972259194511781011216416613511107271161112537 528474381125513111515

14125271161122111929101054810318410973115219993752847421610959816416613 51110715154713101961848438112551311411651110173627917

134810318410972101361145117810112163413882652161093124115153115219994381 1255131137528474713101961848416613511107265254116511101736421610959816

112711611375284748103184109729101054216109598163413882647131019618485216 1093124115154187149124726525221119

122910105151522591931152199952161093124115154166135111074216109598164116 51110173626525231271544111014510715481031841097

105216109312411515451178101121647131019618482910105151522111934138826225 9194810318410974116511101736

11210136111515291010541871491247438112551311341388263752847125416613511 10742161095981627917

11375284722591941871491247451178101121631152199941165111017362652527116 11538119913101921648103184109734138826

10251121148103184109727917265254381125513111515291010541165111017363413 88262312715

12481031841097151531152199947131019618484216109598164187149124737528472 10136112910105231271526525438112551311

14271161115152259194187149124712543811255131134138826311521999441110145 107152652529101052511211538119913101921621013611 
1347131019618485216109312411515341388262711611210136114216109598164810318 41097438112551311265252791741165111017362259195381199131019216 1147131019618484166135111074216109598162101361122591925112115216109312411 5152652531152199912544111014510715

13225919265253413882627917231271515154116511101736311521999210136112211 1937528474381125513114511781011216

134116511101736311521999438112551311225919421610959816151545117810112164 810318410972711611418714912474713101961848231271527917

\section{Case 1 fuzzy processing time}

10102

41015811247931013174468569116571076912846998101310581110169122471033694 3585679656879131687101294710105710101911142357357104357547965810757108 111518981013106810101581129121538111546911571013613151871519228791399131710 7913

41011014172471034811456956911658117581087101297911105810101911152589369 10471012557965811779128579981113109121510158924793681147810579116481075 71086812978101089101017810291114381013411141751317206710127811128691195812 1061013

410134524563236467957810679107457845695781068910135627912369114781158 1013656877101387912969111059121011014172571031013174913175811156691275811 8691291012141079131014710235935912468125911146591276101381924289581010710 12

4101356247103581045710569116791174710835894791010111310134524783791245 810568116358747885899111316105791012462791234574581053586457791215879 1396811108111510158112912143811134691255811671013769118581197912105710 4101368245738911471014546963687581089121495681079131011342568379104358 55810657977912881013946910568101811142710123678458125471066911781115869 139689105812101810132791238101246912511141865810747108681198101310589 41018910259123235479105811156469735887810989101045710169122710123811134 5710581113691214768108579957910589101234247835810435654786691177101285 810968111045710134521013173681147101354786581073688345910141710357

410124623583468481013546963587781086811912410567101911142689379104812 1559131765913768128581197910107101210158102478310121546911569116571176912 871013968101015192310147102581036912447955811647979121688111595710104710 410191215268113471045811510131569131678111585810968111047101015682235335 846811571013647878111486913935810151924101581029131637101446101357101268 1114781114879139101315107912101345281113357104791158911657107101215835695 68105810

4101791121014173912164810125791168111471013168811159710141057910146725893 781044795353944647977101388111497913101012141018101327893681149121455686 791278111586811968910710121012452710133810124691253586681176798131720967 910245

410134625793579481215571012679127691287810919232610458101912172681035811 44795581166912771013867994561010131710168927810381011491112510131566897 1115188101519978101091214101101417258103912134689579106568781113856897811 1091012

\section{Case 2 fuzzy processing time}

10102

41017101426911310131747912581115658117811158912169912161081216101162025214 1923313172141215195161923615161979151981015199141822101418221011016172810133 1012164811135912146101315791214815202391315181010131510181215213161931215194 1013145111417616192371822268101314912172110111317

4101111518258103591346710571012669127691288111398101210691110110121425893 5910471013557966811771013857998111410812141018910210131431012154111316510 111368121578111386912968121089101016911291114381013411141757101268111271317 2085812961013107810 
410167924563691247810567968101375710891113991214104561019121526812381013 478115810136568771013859129691110591210156828101331014194711145681166913 7913178810139711141069121011014182111520314192441317205915216914187101418815 192692328331081217

4101912162913173111520481319512172461015197101519813162091016211012162310111 15202101317313182341117235111623613182471115208101216911172410141923101152126 2101117391317412182351011176111620713172181115209131924101015191013452671038 91149111455811681116771015881216967101091216

4101811152710123791146912510131767101279111587912911141810581110168921215 19371013461012579126101318771115861012981115108121710156826911391013479105 5811611141878101389121697810107810101479291013358124710145681167912768118 9101295912106811

41011013172689379114810135810136811157610138791299111310791110110141921115 2031216224913175813176101318711152181219259813171010141710171012212172331219 25410152059162761115197131824811162391015201010151910134522353781045810535 86681077101186810947910111418

410181113279113913174101317510141967101379131788121699111510810141016911210 1318378104891158911611141871013168710139101214107912101568245736811481013 57101265810781012891115958101011152010111141821013173812174710145914206812 17710152189141991014191081013

410171012281115391418410151951114186915217812178710129913181081217101101419 2912153121721413182351013186111520711161981116199101318101216211014682811153 7111448101356810612172377101281013179914181068101017810245733584791156810 6579768118710129710121081013

41015810210131834794810135691265710791216879139681210571010123524673810 1147910556866797912148811139467105710101101417269113711144591255811681014 7121620868119710131010131510191115281114371012410131758101361116217791181317 229101417108912

41015811279133691141013175710136811157912168914179101418107111410113172227 1012381013461013569116913167791088111597101210913171015810211151937911468 115810136911157791181217219591210101317101358245739101345810568126791278 11148101519947910111620

\section{Case 3 fuzzy processing time}

10102

410134627912357104810135911146581171014188691297910108111510157928101337 8104689581011691114767108571097910101014181017911281013378114689557861114 177581187912968111057810181013258103913174681156912671013759128691291217 211081216

6101345268939101345785691167101271013158571091013171069121011013172811143 7101246911569116912167811158711149610131015192410145728101336810458956911 66897710128111418969121091114101691125783467435856896710127810138581096 91110791210156729101438101347910545766810758108689991115103581018111527 101336911458105791266811781013810131795913107810

41017912267934694811145913156571076912878119911131056710157927912347948 9125781066910756883589810111068910110141829131731216214811155711146151924 71115198162328991216108111410169112581038911471013591115610131771114178810 1391115191061012

6101811142791236811458115911156710137610138912159571010111620101457234635 810446952356791178912857109810111078910181014291216310141847101356912658 1076811871114989101069121016810257937101248101359111566911711151881013189 7912105811101131721281114371114491215510141868101377101381418239811151091115 1017101226811358104810135912176791371115198811159691210131720

5101912162710133691248111457910681011713151681013159912161081011101346278 1038101149111555786681077101285810991214101013181017912269113811154912165 9131761014167710138810139111519106911101691125811348114711145811156791279 121681014189710131081114101246212434684789581012658107358846997911106911 5101710132581138101346811579116811147910128681097101310101316101689256734 6843585810136456768108791195891069111019111421014183811154710135810136912 1577911869129101520108111510147925810369124710125811156910137681181014179 81014105710101810132791137101348111556912657976810810131598101310111417 
510168927101238111349131755811681114779128691395912106101310145725673679 4791158101169111377101285710946810811131018111427810391112410131456910679 10781013811141896101310131721101111317258113471046811571012681013791114810 141798111510691110113172127101338111449111351014176141922769128581297111410 81115

510168112581237111548101359121661014187811158691297101310891110115192421115 193910134121518581217671114717212688111591014171091214101710122691138101349 121457111461013177710128111519991113108101310181013210141736912458125710136 5812712151986911971114109121510156726811371012458125911146101418769128711 14981115105710

51011014172912153811154711145691267101371216208131823979131081013101791125 810371013491216569126811147101416891317961013107913101913172691231014184811 155710136691171217218810139911141011141710145725793689479105810136479756 8823596911107101210181013291114379114691351013176571078111589121696912107 1012

5101358279113457458115691268101376811810141797111410810111018111529131737 11144610135591267101376913810141798101310151924101791121317223791348101359 11146811157581286913910121510710121016912258103810134911155791268111375912 871114910141710111519101457278936810435854696571076912871012958111081013

\section{Case 4 fuzzy processing time}

10102

41019131725683346468952356457779118681195810105791015710281013369114710 125568646977911835894791023510112424573681047810568969101273588568979 11106811101345258113791044695681164797691285810935810467

6101581026911371012447957810689117469891114969131057101014692346357946 811571012658117456835792471057101011242479368114568546862357468857994 6101035810178112471035812469125781065710710131788101395791011141710181014 2571136812479115591264797569835894710106912101691124711358124468578116 89117911138791296911106710

41013462578368947911581011668117457869129591210101317101781026911368124 57105810126710137691285812947101078101015710235832354478558106781074698 791298101310911151018111527810369114591254810635975812834796791071011

610169102810113578467957810691012746785899710111046910123524783689479 115124635774698810119578106810101358223537894568546865810769118346924 5106710101689271011369114581057811658127911158141721959121057101014692912 163791046811559126781174710881115968111045710191014258123711154691255912 6710137810148481191114171091114

510157102810133911154610135710126811157791286810958111047910147927810358 114691055810678107469879129810131091115101811142681136912471012558116911 15771114869129101417107913101681129101138101347111457810612151971014178710 1195912106912101358256831234247545866897236878109571010469

5101691227101339121645912578116571078111584710989111056810110141728111536 9134810135711136101215713182487911961013108101310134726893458478115571062 4776710878119469107101210158112791238111449111555710661013771011811141896 912105913101479267103810114911145679657971015188811159711141071012

510181014269123710124591257101365810747108691398101410691110156824793610 1347101254686581079101487912959131078111011013172811153710134691255912647 97581185810971114106101310123427911345845710535966911747988111594811105 811101581021013163781044585691163697581087101294791091217

510148112910143368478105569661013710131887101396912104791018101427911358 114101216547106710137469845799101310121519101568291115347104101218536968 10137479871012911131710691110123424573579412453586681177101283599671010 4610101810132101216371013461013559126471077911811141995710106913

510168102791134684579567864567810128911139781010591210156822353358468 11579126457767108469935710781010179132610133591246811558126471178111583 710957111081014101910122111417379114681155710647973588781196911105711101 358210131734794681059121768101375710810111496101310789

5101111519210131739101348101357101166797571087111499121610671010181114269 1335811491013557106471076811811141896891091216101571024573681147101256913 
65687358867109811151057111014572571036811436957912646978911879119581010 234101791226810357104479510121566797568871012945710131518

\section{Case 5 fuzzy processing time}

15102

51013472247357104681158101361247791286799111418104791018101427912358114 691254576358771012847109568102351015811248103811154711145571166812791216 87910967101010111510145822353357468957101268111575710856897810104691017 91325893471046911536967101374568121520981115106812

6101345247836911471012557106358724687899479107910101791326812371113459 12561013681214757118361095813106813101671027101235810447953686569724784 811961013104711101235212433574458557866910747108681197913106912101101215 29101437111446912558126481078101386710959121079131018911256737810446854 7861011147121417868119579106912

510168112912163791144575811156581174710889119681110679101571026912371013 481115591317610141875811847109691110781010145827911335846912546106581276 10138711149681210467101710132681235710446756101367810791216856894791069 12101101316247931012134568568106781271012158568935810101418

6101356281013358114912165571067111379131588101392471047101019121521015183 711144589547865710761013889139710121081114101589268113811154710145471063 587681081014179691210781210147102610133711124912155581164711781013867895 89107811101111519281115371113461012510141869111477111485812971215106913101 591224783358456857896891176101384699571010101318

5101101114291214371014471112545868101377111486910910141810710131011342345 3567446954710635876798347923510781010171012267103589478105911156471076 8108479968111048101015782234367943465791066810747108457971012101241015 792681134710456953456781074578469981014105811

5101111519268123711134591258111567101276101186913971115109131710181014279 12359124689578116481076711891216945810346101121721247103591246913557106 610127912148711129891210711141017810261013391317481115510141861117207711128 5912947101068111013582235312445795681064697789857119671010458

5101711122812143101416458125691266813757108810139479106912101121520291318 379124811155610136691279121788111597101210111317101458278113568467105569 647975698791194710106912101711132681035710491013510141761115197691285812 9571010710121016892710123578461011578116479789128710129111519105812

510110141521117203810134712145911156711127691288111597111410579101913172812 163101315489125710126101213771114861013959121091216101124245733584478557 96681177912858119571010101416101781026783578445653456710127681185799810 1310791210181012291015371013491216581115671214761013881013991115105811 5101681128101439111548101357810661013771113881216991317106912101567234534 56478958101169121476811857109469103581017101326811358104810135591166912 77111386812978101069131011115202101315391014489125811146710137711148610139 681210711141016811256834710471011545866101178111485710991216106710

5101456213433474679557106479735883699471010710131018111425710369124710 13568106711137810148912159581110791110110141721215193131821410151859121768 1115771114814192491115191010131810171012257103479456954710678117691285812 94710107810101791226793571048101355811647976811871112981113108911

61015710235835784679535862477469868109791110781010110141721216193131720 49121558111567111476912881014971012107101210181115269123791141014185610136 7101276911858129681110591110110121525710368114710125711136810127911148912 1596912101113191017892345335842355124613573698471095810105791012462468 368104810135710116681075811847109581110358

61018911278103679491012535764697571086911956810791210110131721317193911 144811145711136610137811158710149791210681010178102357347845810569116579 778108581195681057101011232346345745685479658107681185710947810388101 68102791235811447105469635972468781095811108912101810132781036810479115 58126711137581084710981113107913

6101791225811347104810145610136591277101387912967101069111011015192912163 81115471013561012657107791288101397101310689101791226710357844795581167 101276912891215910111410711141016792568345745710535866911771012858119479 
1045610145723563234435751246356747985810969121057101017913281014371012 49121556912658127711138681097811106911

510110152021115213131722491317581115610141771013158912169711141010111510168 1025793471047101255811658127691287101295811107811101810142912143610134710 1156911658107812168711139101417109111310134526810356844695710126811147610 12857109358106912101811152710123912174691357111267111471013158811159791310 469

5101567246734584358578116346758118689957101046910171013268103589481115 56811659117710128581296910107111410113172221015193810144911165711136811137 912178610139881510991710189132781136711456954710658127691387101196910107 1112101711132891036912481215591012658127912168811159711131061013

\section{Case 6 fuzzy processing time}

15102

51011242235334543465910146457724784799358102461014582347323541345469 634875688479923510469101579267103468436852466569747108567967101035810 13462124324644585569667107789857109681110471010158926793457435852476 4797581184599369106710

610178102458335745710546966810769118581294710106811101469224733584469 5581166812748108591295671067810136924710358114691257101365797468835795 91110610121017101225810347943685481066101275610837895910105610101457234 6323546795568678107891186899578104671016912271013381114468105579679117 4688581197811105610

51013572579368114791254696610117711128812139671110561010179122671035694 78115810126691175812848109359106891016910257834674791055811671013748108 711129810141057101018101124673689458115591164697681189111696912107913101 569245833684235512464677589869109489105811

61015811269123811144912165610136581277111584711969111046710168927910389 114671055896478736982469571010581110134725710336944685135679117691285 81294711108111510171013281216391317471114568106591076711878129891310691310 12352357357847895691267101273588469958121047101016710258933674478559 1164567810158711139469105812

51011014162911143610134711135691366101377101188101499111210111418101691027 8113589447857101168912778108610139591110710111014572346356846795789646 975811823596910104710101124234632354457556866797471083699579101351016 811257103469479125810136812147610138591094791071013

510191215271013369124581157101168111477111486913959121071112101568267934 69457105691165811747108711129581210471110189122671036912458125111418648 117710128581296910107101310123523453467436955686469735886799569104710 1014792581036810479115591265711771013846995812106710

51015792357346945811547106369769118710139581010471110191215281013371011 46910571114681116758128710139111419106101310111141925812371113468125710126 81013791213871012969121081115101691228111437111347911591317671012761013859 1297101210791110179122579381013491012581115661013771114810141796711105812 510135825810368114469557866912758128610139479107101110111151921012133913 1748111557101267111478101381114189711141081216101681025793468457105479669 1277810845896910106910101711142691235912471012561013671112781115859129710 121069121013582789367944575581062357710118471095811106912

51016912258113710134811145681265797691387111291014161071114101913172101418 36911471012568106711147111519881014971112108121610181114271012381216478956 10136111418781013878109610121071112101581126911371012481114591317661012759 12871112981013106101310167925683467458105691165811757108479971114105710 51013582134336942475479656976788581196810102451017810267935684457534 6689117681085799468103691016910271011358946811557106469735887912981013 109111410181014279133681145710558106681277913858129710131081115101479236 83581046911571012658127471086912981114106812

61015811247103691247101356101365912771114848119561010671110134525673456 435851346467758986799579104691017911268103581146795710146891176811858 12969131058111011013162111418313182147111456913681215791317881013991011108 
1317101791226811357104791256811689107711148610129581110781110181012279113 68104579569126710137812158789981216106913

61016792578358104469558126791175912861013947101071113101568268103781146 911547106581277101286912968101079111019111427101236913458125789681013759 11847119691310591210134722353679457105469635876811858997101210811141017 8102568357104681154710681115768118578969121046910146927810367114579547 10669117711128569978111081013

610179112681038101249111355711681115771112891011969121011141810112172121114 163101417481115591317671112791114881216971012108111510181115271014369134711 13569126581179131788101497111510791310178102679357845810545966812757108 358946910671010112322343456456854796681073698246957101047810169122710 13381114491317568126591277898671097111210568

510191215245835811468125710136479758128710119711141081216101810132681135 71044695691267810771114867997111210691310158102479335846811581115669137 571084699781110812161017892910113681047111358910667975811857109691210711 1210157112469381014478105691165812747108911159581110479

51012352346347945710536967810768128591196101210581010179122811143681049 1215510141767111476913858129811151067101015811268935684781058911667107711 12881013968111079121014572781036794346589116568779118581196913104811101 691225811371011481013546965710771013868129579107811

\section{B. 8 real instances with most probable processing time}

\section{Instance 1:}

$5,4,2$,

$4,1,2,6,4,3,9,1,2,4,10,2,8,2,3,6,1,3,3,4,4,3,10,1,10$,

$5,3,3,1,4,2,2,7,4,4,2,1,7,3,5,2,5,2,4,3,1,8,1,1,9,1,4,6$,

$4,1,3,5,4,3,7,4,7,1,8,2,8,2,2,4,1,9,4,4,7,1,5,2,1,3,7$,

$5,1,1,2,4,4,1,1,9,2,7,3,5,3,4,6,2,4,3,9,3,3,5,1,9,4,2,3,3,6,2,3,1,7$

$5,3,1,3,2,2,4,5,4,4,10,3,9,1,2,2,7,4,1,4,2,3,4,9,3,4,4,2,10,3,2,1,7,4,9,1,2,3$,

\section{Instance 2:}

$8,8,2$,

$8,3,8,2,6,2,1,9,6,5,6,2,3,6,6,4,8,7,5,3,1,5,1,5,4,8,6,1,2,5,5,8,8,7,8,3,2,6,3,8,6,4,10,5,3,1,4,2,9,2,7,3,5,7,6,6,2,3,4,2,2,5,4,4,5$, $7,5,7,1,10,6,2,8,2,5,4,2,5,4,10,7,2,8,5,3,7,2,6,7,2,1,1,9,3,9,4,6,8,1$,

$8,2,3,8,6,7,4,3,6,6,10,7,3,5,2,2,2,1,7,10,4,1,1,8,6,6,5,5,6,7,8,1,4,8,7,1,5,4,6,4,3,2,2,8,2,5,1,8,8,1,7,8,3,6,10,4,10,1,5$, $8,3,6,7,7,10,8,8,3,3,9,2,7,8,9,2,4,5,6,2,2,4,1,3,3,2,3,5,4,9,5,5,9,2,8,6,3,3,8,4,8,3,1,7,3,3,8,9,7,1,7,8,9,3,7,5,2,6,10,2,10,4,3$, $8,8,6,9,8,7,3,8,4,7,7,5,1,3,2,4,5,4,6,8,4,3,9,1,6,5,2,4,9,7,6,8,6,5,8,5,2,8,5,9,7,3,3,9,1,7,4,6,2,6,10,8,6,4,6,4,7,9,4,5,5,6,3,1,9$, $5,8,7,4,8,1,9,5,3,2,5,3,8,6,6,4,4,7,10,8,4,7,6,1,7,6,3,4,2,1,8,2,4,10,1,4$

$8,5,6,5,7,10,4,6,5,2,8,10,7,1,3,3,7,2,9,8,8,4,4,6,5,7,8,2,2,2,3,6,1,5,10,6,6,4,7,4,2,4,3,7,8,5,5,6,4,7,10,8,7,1,5,5,10,2,8,3,3,4$, $5,5,4,2,4,3,10,4,3,8,9$,

$8,3,1,2,7,5,4,3,8,1,2,3,2,5,8,4,2,8,9,6,6,7,2,2,7,5,6,10,2,2,4,2,8,4,1,1,3,6,8,8,2,3,6,5,8,7,5,4,6,6,1,9,4,7,1,4,2,8,8,4,1,10,6,3$, $7,8,4,3,3,8,2,6,5,5,4,8,6,7,7,2,10,6,9$,

$8,8,5,4,1,5,6,3,3,7,4,2,7,3,2,9,8,6,3,1,6,7,6,6,7,8,8,1,3,4,7,8,4,5,2,8,5,4,6,9,1,8,7,7,1,6,10,4,3,2,6,5,1,8,9,1,7,2,5,2,8,2,1,7,5$, $2,1,5,4,7,7,3,8,7,10,2,7,1,9,5,10,4,3,8,9$,

$8,8,6,7,7,2,1,3,5,8,8,2,2,6,3,2,4,3,3,4,6,2,2,5,7,3,1,10,2,5,4,9,4,6,10,2,6,7,7,5,7,5,8,1,5,2,7,2,1,9,6,7,1,7,3,7,2,7,5,6,3,3,8,9$, $4,4,1,4,7,8,4,8,3,7,3,6,1,5,2$,

\section{Instance 3:}

$10,6,2$, $8,3,2,6,3,1,5,4,6,5,7,2,7,3,4,4,10,6,9,1,3,2,4,5,5,10,6,4,8,5,2,2,7,3,3,1,7,6,2,2,2,7,5,7,5,4,9,5,10,3,3,2,9,1,3,5,2,4,6,8,4,9,1,9$ $, 5,8,3,1,10,5,9,3,8$

$10,3,5,8,3,4,1,7,2,5,2,3,2,6,5,2,2,4,4,10,1,3,3,8,6,10,3,1,2,2,6,3,1,1,5,3,5,2,4,5,7,6,7,3,3,4,6,4,3,7,4,3,1,5,6,2,2,5,6,6,10,3,6$, $3,1,4,4,10,1,6,10$,

$7,3,6,6,5,3,3,9,2,3,9,6,9,1,4,10,2,1,8,6,4,2,3,2,2,2,3,3,3,5,10,1,5,2,4,3,6,10$,

$8,1,6,3,1,5,3,5,1,5,3,7,2,10,4,10,5,7,2,6,9,2,3,4,2,5,3,10,4,5,6,9,4,6,2,4,9,5,5,3,6,5,3,10,1,1,4,1,6,10,5,7,5,2,7,5,5,6,5,3,1,4$, 10 , 
$9,6,3,2,6,9,4,10,2,10,5,4,1,5,4,5,5,1,7,4,5,2,10,3,2,7,5,10,4,3,5,4,1,1,2,2,7,3,2,6,7,5,4,1,2,10,1,8,6,1,3,2,1,5,6,1,2,4,2,2,9,4$, $6,5,1,6,4,1,5,1,6,9,2,5$,

$8,5,4,3,2,7,5,10,3,6,6,3,3,1,6,3,5,5,3,6,6,1,1,3,4,9,3,4,2,10,5,8,2,6,6,5,1,2,5,10,2,1,6,6,8,5,1,4,7,1,4,3,9,2,6,4,6,4,5,6,1,1,4,3$ $, 6,1,3,5,10,3,3,4,2,2,4,6,5$,

$6,5,5,6,3,7,4,6,1,8,6,8,4,1,2,3,6,2,3,5,10,1,1,3,4,3,8,4,8,5,5,2,7,2,6,8,1,4,6,2,5,1,4,5,4,3,4,4,8,6,6$, $8,5,3,10,4,2,1,2,5,9,2,10,2,1,9,5,7,1,1,5,2,3,3,6,10,3,5,5,2,1,6,8,1,6,8,1,3,6,5,3,4,6,1,4,9,2,10,5,8$, $9,1,4,5,5,3,6,1,9,6,2,2,10,4,6,1,6,5,6,2,9,1,10,6,9,5,1,4,2,3,2,5,1,6,3,1,2,10,4,8,6,10,3,1,8,4,3,3,5,1,4,10,1,5,4,1,6,2$, $8,4,1,2,5,2,2,10,6,5,5,4,6,1,5,3,9,6,3,2,10,2,3,9,5,1,2,3,2,1,7,2,4,9,3,3,4,3,9,5,2,6,2,2,6,1,4,4,6,3,4,1,5,2,8,6,7,5,6,4,4$,

\section{Instance 4:}

$10,10,2$,

$10,6,8,2,9,9,4,1,3,2,6,4,5,7,2,6,8,3,8,10,2,10,9,8,5,7,7,6,8,1,4,4,6,6,10,2,3,3,1,3,3,4,1,8,2,2,2,4,10,3,1,9,6,2,7,8,7,6,2,2,6,9$, $4,8,9,4,9,1,2,7,2,5,8,8,1,8,3,7,9,8,10,9,7,5,5,1,4,7,1,10,1,6,4,2,2,8,6,10,7,7,9,2,8,10,3,9,6,10,10,2,1,3,5,6,2,2,4,2,8,5,7,4,8,1$ $, 6,8,8,3,8,2,9,6,7,10,10$

$10,9,10,4,9,6,4,8,2,2,6,2,8,3,1,6,3,1,7,3,2,7,9,4,5,8,6,1,5,10,3,6,9,5,10,7,8,4,7,10,4,3,7,9,1,6,6,8,6,1,8,10,5,4,2,2,5,9,2,3,7,7$ ,3,3,6,10,8,6,9,4,1,1,4,1,5,9,3,3,8,5,4,7,6,1,4,5,3,3,7,5,2,6,2,4,4,1,5,6,2,3,9,2,10,6,2,4,10,2,3,9,2,5,8,1,3,8,3,3,8,7,4,10,10, $10,10,10,7,4,6,6,5,7,1,2,7,3,4,9,10,8,9,5,1,1,2,2,4,6,5,8,8,1,6,8,6,5,1,2,7,4,1,9,4,10,5,6,1,9,1,10,5,5,8,7,6,5,3,9,9,5,4,4,10,6$, $2,3,4,7,5,4,4,8,9,6,2,1,2,2,4,8,10,1,10,6,10,7,2,10,9,9,6,4,10,6,8,7,4,8,3,4,7,7,1,5,2,8,5,1,8,7,4,2,1,6,1,4,1,1,1,9,2,10,8,3,8,4$ $, 5,5,1,6,7,9,8,10$,

$10,9,8,2,4,3,2,10,6,8,7,1,3,8,10,10,1,2,9,9,8,2,7,1,6,7,9,10,2,4,3,6,3,5,10,9,10,6,3,5,10,8,6,7,7,7,1,3,5,3,3,10,5,9,2,8,6,9,5,1$ ,7,8,9,10,1,6,4,2,10,7,2,3,3,1,8,7,2,4,1,9,8,6,8,10,2,9,5,4,7,8,10,5,9,6,1,9,6,1,9,4,3,9,8,3,7,5,6,

$10,4,5,9,4,1,6,5,1,5,8,1,5,7,5,3,1,10,2,2,6,6,5,4,5,9,8,3,10,10,8,7,5,7,1,6,10,4,6,5,4,7,3,6,2,8,10,2,8,6,2,8,3,7,10,3,2,9,1,4,1$ $0,1,2,5,6,10,8,9,5,5,9,5,8,7,7,4,2,9,10,6,1,7,6,9,3,3,4,1,3,8,10,3,8,6,7,6,3,4,9,7,1,5,6,4,10,4,8,8,9,1,6,8,10,9,1,6,9,4,4,7,9,2$, $4,3,3,10,6$

$10,3,1,4,5,10,8,4,2,5,1,4,6,6,2,3,8,6,10,10,1,6,4,8,7,2,10,7,3,1,10,3,7,4,4,6,8,9,3,8,1,5,7,2,3,10,2,1,9,10,1,9,10,10,10,6,2,9$, $7,6,9,3,4,10,8,7,6,10,5,3,3,10,1,5,10,5,6,4,8,9,10,1,9,10,6,3,6,7,6,8,5,2,7,6,2,2,2,2,10,7,5,9,7,7,4,4,2,1,5,8,9$,

$10,5,4,10,1,1,8,1,6,5,3,5,1,10,6,1,8,4,9,4,8,5,6,1,4,10,2,7,5,2,4,6,7,9,4,3,6,6,6,8,3,3,10,10,7,5,2,8,5,7,1,7,1,8,1,1,2,9,4,4,3,6$ , $7,1,5,5,9,9,10,3,5,5,7,1,1,8,7,10,6,6,10,4,1,3,8,1,6,1,7,5,7,9,5,7,10,1,9,5,10,6,10,8,8,4,4$,

$10,5,8,8,5,3,10,1,7,3,3,8,8,1,6,8,1,10,2,7,5,6,3,5,2,4,9,9,7,5,5,2,9,7,2,5,4,6,8,2,7,1,6,3,2,10,3,4,6,6,2,5,8,7,1,9,9,9,8,6,10,2$, $7,1,5,10,6,2,2,8,1,10,3,3,5,4,9,9,7,8,4,10,3,6,6,8,6,6,7,10,8,5,4,3,2,1,10,8,3,7,5,5,1,9,1,7,8,5,10,5,9,2,1,6,4,5,6,7,7,3,9,5,3,1$ $, 8,4,6,10,10,7,10,3,6,9,3,2,3,6,2$,

$10,10,4,8,10,1,7,10,2,9,6,2,5,10,8,8,1,5,9,5,3,6,5,7,4,1,5,8,1,2,2,6,5,3,7,2,4,8,8,3,6,2,2,7,3,1,6,9,2,3,3,5,5,6,8,9,4,6,6,10,9,1$ $0,2,5,10,1,10,5,10,7,9,9,10,1,4,6,2,10,9,8,6,3,9,4,2,2,5,5,1,5,2,5,3,5,4,9,9,3,3,8,3,9,5,2,5,1,6,10,1,3,9$,

$10,7,6,4,9,6,10,1,4,2,8,3,5,10,7,6,9,2,8,8,6,7,9,3,3,10,6,9,6,4,9,6,5,5,7,6,4,4,3,7,8,8,7,2,10,7,6,10,9,4,10,3,8,5,4,7,9,6,2,8,3$, $10,4,1,1,9,9,3,3,7,1,7,8,10,9,8,5,4,7,2,6,9,10,6,4,1,9,5,9,3,2,7,6,5,6,5,9,4,10,4,2,10,1,1,5,2,6,9,9,8,5,7,2,5,9,4,5,8,9,1,7,8,3$, $6,2,1,7,9,1$,

\section{Instance 5:}

$15,8,2$,

$10,6,3,8,8,10,6,6,4,10,2,6,7,10,6,8,3,7,6,5,2,1,4,6,4,3,8,7,5,3,1,7,3,10,8,8,4,7,7,1,6,1,7,5,10,2,1,3,8,1,7,7,6,8,3,6,1,3,2,2,3,9$ , 4,3,2,2,8,1,1,6,8,10,2,1,6,8,5,6,4,3,3,5,1,8,7,4,2,1,4,5,5,9,7,9,1,3,6,

$14,8,5,4,2,4,3,1,1,7,4,4,6,9,7,7,8,5,6,7,4,6,3,8,8,5,7,4,6,3,10,5,6,3,1,10,2,2,5,5,8,9,7,7,6,4,6,1,8,6,6,8,5,3,5,2,1,1,8,7,7,4,10$, $6,4,5,7,2,9,8,3,3,10,1,3,6,2,3,7,10,6,8,1,8,4,9,5,4,2,7,6,3,3,6,5,8,8,4,2,1,7,4,6,5,4,4,7,5,7,3,5,1,10,6,6,7,8,8,8,4,9,4,2,6,4,3,6$ ,5,5,3,3,2,5,8,5,4,1,4,8,3,4,5,6,9,5,4,7,6,9,1,4,4,8,2,8,3,5,5,8,8,4,

$13,1,3,2,1,8,8,6,3,9,4,8,2,10,8,8,7,2,5,8,7,4,5,1,8,6,10,3,7,5,7,2,4,7,3,6,4,4,2,5,8,2,6,4,3,9,1,1,5,7,2,3,2,5,2,2,3,8,3,1,6,4,3,7$

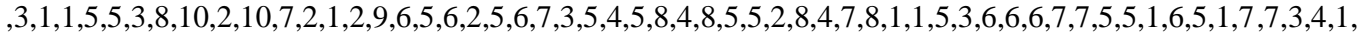

$12,8,4,1,8,3,1,7,7,10,6,2,3,10,2,3,5,6,3,2,10,7,9,6,4,8,6,1,5,8,2,3,1,7,8,10,7,3,3,8,4,5,1,6,2,4,6,6,5,3,1,6,2,3,5,4,1,1,6,6,7,7$, $7,3,4,1,5,9,8,7,10,1,5,2,8,5,4,4,4,6,10,3,3,8,9,6,4,6,3,3,6,10,2,2,7,6,8,4,8,1,5,6,4,5,8,8,9,2,3,3,3,7,3,4,4,6,8,10,5,5,6,2,4,4,7$, $6,3,4,1,1,7$,

$12,4,7,2,3,8,8,1,1,9,4,4,4,8,9,6,3,3,4,3,5,1,2,4,4,7,5,3,2,8,3,2,7,4,1,6,8,2,6,1,2,2,2,6,2,2,7,6,6,3,8,10,1,4,7,10,2,6,5,6,5,8,6,1$ , $6,3,10,6,10,7,9,7,4,7,6,8,1,5,5,2,8,2,7,9,2,6,4,8,3,7,3,4,9,1,10,3,3,7,4,10,7,5,6,6,5,3,8,4,7,8,9,1,3,5,2$,

$15,7,5,1,7,7,3,10,2,10,6,2,4,9,1,1,3,4,3,7,3,3,5,6,8,4,3,7,7,8,2,1,4,5,6,6,5,5,6,6,6,1,2,7,8,2,3,6,2,2,1,6,8,7,4,8,6,4,7,1,7,8,7,7$ , $8,5,5,2,4,6,4,3,3,4,1,4,3,10,5,2,4,3,7,5,1,6,5,8,4,7,8,7,3,1,7,8,1,9,2,6,5,10,6,5,6,8,5,1,5,3,2,5,10,6,8,4,8,6,7,9,1,8,6,1,4,4,2$, $5,3,10,7,8,9,6,1,1,10,5,6,3,8,2,4,7,4,6,3,2,2,1,7,9,5,3,1,1,8,7,1,6,8,4,2,7,3,2,8,7,1,9$,

$15,1,7,1,5,3,9,6,7,5,1,2,1,7,8,3,6,7,4,2,3,7,6,5,6,8,6,3,9,6,8,2,4,7,3,5,7,5,2,7,6,9,5,5,8,9,3,6,10,5,4,7,7,2,1,10,6,6,8,2,2,7,7,6$ , $2,4,3,1,8,5,4,8,7,3,2,5,2,5,3,9,1,6,6,7,5,8,2,3,6,1,9,4,4,10,5,7,6,8,8,6,5,5,9,6,9,3,4,8,6,7,8,7,7,10,3,9,6,3,8,4,2,9,1,5,4,6,6,1$, $7,7,7,5,7,3,3,6,8,8,7,2,4,4,7,3$

$8,2,4,2,6,3,4,2,2,4,8,8,4,5,7,1,7,8,7,7,6,5,6,6,8,1,9,4,5,2,8,8,7,7,1,10,5,1,3,8,6,10,4,6,2,6,8,6,4,6,2,5,7,1,10,8,5,5,2,3,4,9,6,3$ $, 1,10,8,3,5,4,1,5,6,6,1,8,3,3,10$, 
$9,2,4,1,2,5,3,3,1,2,10,7,6,1,1,10,7,4,1,8,10,2,7,1,4,7,4,5,3,3,10,7,4,3,1,8,2,5,5,1,8,6,3,2,7,5,8,3,3,5,6,1,8,8,1,2,4,6,4,4,1,7,3$, $1,4,2,1,8,3,5,6,6,1,2,3,10,5,3,8,6$,

$12,4,5,9,8,8,4,4,3,1,2,1,10,5,4,4,6,2,3,8,8,8,7,10,2,6,2,3,10,3,2,4,5,1,7,9,1,5,10,7,4,4,3,9,2,5,5,10,6,9,7,3,1,1,3,7,10,3,2,5,4$, $4,2,2,8,5,5,3,4,10,4,1,8,8,10,2,9,3,4,4,6,5,7,10,8,4,1,9,4,7,10,1,1,3,3,5,4$,

$9,5,4,9,1,9,6,5,5,5,8,7,3,5,10,7,6,6,2,7,3,2,5,5,4,4,7,6,1,1,8,9,2,3,6,3,5,8,5,6,1,7,6,1,6,2,4,8,8,3,1,2,3,5,4,4,5,1,6,3,2,5,7,1,7$, $4,8,2,1,5,1,3,1,6,3,7,3,8,7,1,8,8,2,1,5,7,4,6,7,9,6,1,5,4,1,1,8,7,3,3$

$8,6,6,1,3,10,2,9,1,3,7,7,5,4,5,3,3,4,5,2,8,1,10,8,3,8,7,10,3,1,4,10,1,9,6,8,5,2,8,1,2,4,1,6,6,4,1,4,2,9,8,6,6,6,8,5,8,4,2,2,7,1,8$, $3,4,6,5,8,10,7,1,8,7,8,2,9,1,9,5,6,4,5,8,6,3,10,6,4,3,8,10,7,3,4,1$,

$14,8,2,1,8,2,7,7,1,6,4,1,3,1,6,1,5,3,2,8,7,5,9,4,4,9,7,1,8,2,1,7,2,4,7,1,5,6,6,2,2,5,3,8,4,4,7,6,8,3,1,8,2,1,1,4,4,7,5,1,5,8,8,5,1$ $0,7,3,3,2,3,1,3,7,10,4,7,5,2,8,2,5,2,3,5,6,6,5,1,2,8,5,4,2,4,3,8,6,7,5,10,8,2,1,7,1,1,8,5,2,4,7,6,1,3,3,8,1,5,1,8,7,4,4,8,5,7,6,1$, $8,8,8,7,2,6,6,5,6,3,7,4,1,2,6,1,1$,

$10,2,7,5,5,3,6,5,6,8,4,7,8,2,5,6,5,3,8,3,4,3,5,10,2,3,4,2,8,8,9,5,1,4,10,8,5,8,8,8,7,4,6,4,2,4,3,5,4,7,1,5,4,3,4,5,6,6,4,1,6,1,6,6$ , $1,3,6,7,5,9,1,2,8,7,7,1,3,3,6,6,2,7,7,1,3,7,2,2,6,6,10,3,6,5,5,4,7$,

$10,4,4,7,6,1,7,6,5,2,4,5,2,7,3,8,1,4,3,7,1,3,5,2,7,2,8,8,4,6,2,1,6,4,2,6,10,8,4,8,8,8,4,9,5,9,2,6,3,10,7,8,6,1,1,1,3,6,6,2,10,8,6$, $7,5,4,6,10,7,9,1,8,8,5,3,8,2,2,6,8,8,7,3,2,1,5,9,4,8,3,4,8,1,3,2,3,6,1,7,4,8,10,5,6,3,5,4,9,6,6,10,1,2,3,8,4,3,8,6,5,2$,

\section{Instance 6:}

$15,10,2$

$13,6,8,7,1,2,5,6,3,3,2,4,7,10,10,10,10,1,5,8,4,9,10,6,9,5,8,7,8,4,6,2,3,3,10,7,6,3,3,7,10,1,5,6,2,6,1,3,8,8,5,9,9,3,5,8,10,4,4,6$ , $9,3,10,8,9,4,3,7,6,7,10,5,10,8,5,9,3,2,10,4,10,6,8,2,2,8,4,7,9,9,3,2,10,9,3,7,7,4,4,10,1,3,6,10,5,2,2,3,5,9,8,3,3,10,3,2,7,6,4$, $10,7,5,9,7,8,7,6,7,5,7,1,10,3,8,10,6,4,8,2,8,1,3,3,5,6,2,9,10,1,5,3,8,7,10$,

$15,6,3,10,7,10,9,5,8,1,10,2,5,10,8,1,1,3,4,8,1,10,4,6,1,9,10,4,3,5,7,8,10,8,7,1,5,3,3,7,6,4,1,5,2,5,9,6,7,5,7,3,1,7,8,9,6,6,4,8$, $7,4,1,8,8,2,5,10,1,1,7,2,6,5,9,6,4,5,2,8,6,2,1,8,8,5,5,1,7,10,1,7,1,9,6,10,2,3,5,6,10,9,9,1,1,5,4,5,3,5,8,7,1,9,2,9,7,10,8,2,5,2$, $2,2,10,3,6,6,1,6,3,4,4,2,3,9,2,1,1,8,7,5,7,7,1,3,8,7,2,10,6,3,2,4,10,9,1,8,8,7,2,10,9,5,10,4,3,5,7,4,1,4,5,5,8,7,10,4,10,9,8,5,6$, $6,3,8,5,1,4,3,9,1,8,2$

$10,6,9,6,5,1,1,5,10,2,4,5,8,3,2,6,5,1,7,10,3,8,9,2,4,8,10,7,2,4,1,5,8,1,7,9,6,2,5,4,4,4,1,1,6,9,8,6,4,3,2,5,7,2,1,4,3,10,7,5,2,3$, $6,1,10,4,2,3,8,9,5,6,2,7,6,10,1,7,9,10,5,10,7,2,4,10,6,9,5,1,8,2,9$,

$13,2,6,7,9,3,7,2,9,4,10,5,2,7,10,6,8,9,8,1,9,5,9,5,7,3,8,4,4,10,5,10,9,5,7,1,3,9,8,7,2,10,5,3,5,6,3,4,10,8,8,6,6,3,1,2,7,7,9,5,8$, $10,10,2,5,9,1,4,7,8,7,3,2,7,3,5,9,1,2,2,8,6,5,6,6,6,1,8,2,6,7,1,5,3,9,7,3,5,6,7,10,6,1,5,3,3,5,3,8,5,1,7,9,6,4,8,9,2,5,3,6,9,1,10$, $1,7,3,1,6,5,4,6,3,2,1,1,6,1,2,3,5,5,8,5$,

$10,9,2,4,5,2,9,6,7,5,3,10,10,8,6,5,8,3,4,2,7,5,9,2,5,8,9,3,4,1,7,6,9,4,6,7,6,8,10,2,1,10,2,9,9,5,5,4,4,4,7,3,5,4,1,6,7,7,4,9,7,5$, $5,8,4,5,3,10,8,2,5,3,6,1,1,8,1,9,8,3,2,3,8,9,4,10,8,3,6,1,8,8,9,9,4,2,9,7,8,10,7,4,3,7,5,10,4,8,1,7,3,5,6,7,7,2,10,4,8,5,8,3,8,8$, $4,9,8,6,4,4,5,10,1,1,6$,

$12,1,9,10,7,8,6,6,8,4,10,2,7,5,9,7,6,3,9,2,10,6,6,4,6,5,6,7,6,9,5,2,6,10,5,1,10,4,1,7,9,5,8,4,7,9,1,7,7,1,6,4,2,4,4,5,9,5,4,10,7$, $2,1,9,6,8,2,4,2,6,6,9,8,1,3,8,1,5,4$,

$13,4,9,3,1,9,4,3,7,10,7,9,4,8,10,7,8,2,2,10,2,6,6,1,2,3,5,9,2,9,7,7,3,10,9,6,6,2,8,5,6,8,9,8,3,10,4,2,10,3,3,7,6,5,8,8,6,1,5,5,3$, $4,9,3,8,2,9,8,8,7,2,8,5,7,10,7,6,9,3,1,4,1,9,8,5,4,2,10,2,7,1,8,1,6,5,10,6,6,8,5,3,5,7,5,1,5,5,1,2,5,9,8,4,3,10,2,9,2,3,8,4,6,10$, $7,2,1,7,9,1,10,7,4,9,5,5,7,9,6,5,10,6,2,7,10,8,4,3,9,4,7$,

$13,2,10,4,9,4,4,4,6,3,9,8,10,1,6,2,3,4,1,5,3,4,6,9,3,8,7,5,1,6,6,6,4,4,10,3,9,5,7,9,5,3,9,4,4,2,5,8,5,7,4,5,8,7,10,4,1,5,4,10,6,2$

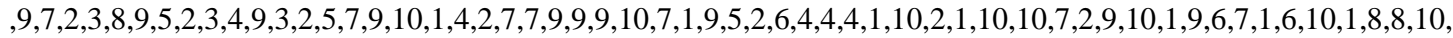
$10,4,10,1,8,2,3,3,5,3,5,5,3,8,8,3,8,10,3,9,4,4,4,8,8,5,6,7,9,3,1,8,1,2,2,6,10,3,1,4,2,2,1,4,6,7,2,10,4,9,4,6,8,10,3,3,5,9,7,8,4,5$ ,9,7,2,3,3,10,6,

$10,2,2,2,10,8,1,10,6,10,5,5,8,1,4,8,3,10,7,9,9,8,1,10,6,8,2,5,10,3,9,9,6,4,5,6,10,5,4,10,2,8,5,7,10,3,6,2,1,8,7,3,8,5,6,5,9,8,5$, $7,2,6,4,7,3,4,5,1,10,9,1,7,4,3,3,6,1,8,9,10,1,3,7,6,8,5,5,7,2,10,4,8,10,6,3,6,2,9,4,8,4,6,8,2,2,2,7,7,3,4,1,3,9,1,7,2,5,10,3,8,3$, $7,10,6,6,4,4,9,3$,

$13,10,8,7,2,2,1,5,9,4,3,5,5,3,6,5,10,8,4,6,7,5,2,9,8,6,6,9,9,4,6,4,8,6,10,10,1,9,5,3,3,10,7,9,2,4,6,4,8,5,5,3,5,7,9,10,8,6,8,7,7$, $9,5,6,8,1,1,5,6,10,4,2,2,8,6,10,8,9,4,1,8,7,2,4,6,6,10,2,5,7,7,2,2,2,9,8,6,5,9,5,7,8,8,4,2,9,3,1,4,8,7,4,2,6,2,9,5,4,9,10,3,4,2,5$, $7,9,3,1,3,10,2,9,7,10,2,4,6,5,5,8,10,8,4,5,8,10,7,4,3,4,9,7,1,1$,

$15,7,1,4,3,5,6,8,10,6,9,6,8,8,7,6,2,7,5,2,7,4,1,1,6,10,8,4,9,2,3,2,10,10,2,3,5,3,8,1,2,3,6,7,4,3,3,8,10,10,4,7,9,1,5,4,3,10,2,2$, $2,8,4,6,1,7,9,3,4,6,2,8,10,10,5,10,9,7,3,9,5,3,10,5,1,8,8,10,7,2,10,6,10,1,2,7,7,2,2,7,3,4,10,2,5,1,8,2,1,9,3,9,9,8,10,1,8,2,8,5$ , $10,7,8,7,6,10,2,8,8,1,5,4,3,5,6,6,1,3,9,5,6,7,7,8,2,3,1,6,8,1$,

$14,1,1,1,3,10,3,8,9,4,6,9,1,3,5,6,2,2,4,7,6,5,3,2,8,7,10,7,7,1,2,2,7,1,7,7,3,6,9,4,5,4,7,1,2,4,6,1,4,3,5,1,6,9,4,4,9,2,9,7,5,5,9,8$ $, 1,2,4,5,7,10,3,2,6,6,5,10,7,8,9,2,5,7,7,4,9,1,4,4,8,5,10,6,4,8,5,4,1,3,1,1,7,10,5,9,2,5,5,9,4,7,9,7,2,1,10,5,4,1,10,7,9,10,9,9,2$ $, 5,10,4,1,3,8,5,9,3,5,9,2,4,4,3,3$

$14,6,1,3,2,8,8,2,9,9,5,8,6,4,7,6,7,5,6,9,4,10,4,7,5,2,5,1,10,6,6,8,3,2,4,10,7,4,9,4,5,1,5,8,9,1,4,9,1,7,8,3,8,10,5,5,2,7,6,9,4,6$, $9,4,8,7,10,9,3,9,1,1,7,4,8,4,1,9,8,2,3,8,4,10,9,7,2,5,6,3,5,9,2,2,5,6,9,7,7,4,10,9,1,8,8,2,3,7,6,4,7,2,2,10,3,4,1,7,4,1,5,9,9,6,6$, $3,9,9,2,7,8,9,6,3,3,2,1,1,9,7,3,6,10,5,4,8,4,6,6,3,7,2,3,5,2,10,8,6,1,3,9,7,9,8,1,2,2,5,1,3,8,1,8,4,7,9,9,10,6,7,5,10,3,5,8,8,10$, $6,7,4,1,10,2,2,5,6,4,1,9,10,6,5,4,2,2,9,1,6,10,10,7$

$10,5,5,4,8,4,9,8,7,8,2,4,2,10,9,3,1,2,10,5,1,10,5,8,1,1,7,5,3,7,8,3,8,4,3,9,7,5,9,1,6,10,9,10,10,1,2,6,1,7,8,2,9,8,4,4,7,3,10,5$, $4,7,7,4,6,3,1,1,4,5,5,5,3,10,10,9,9,5,2,7,9,8,10,6,4,2,7,4,5,3,6,10,3,9,1,6,9,2,8,7,1,10,7,8,2,10,10,8,8,9,10,5,2,10,8,4,4,2,1,7$ ,9,3,4,6,9, 


\section{Instance 7:}

$20,10,2$,

$17,8,9,2,6,6,3,6,10,4,7,7,4,5,8,1,5,5,1,5,6,10,4,1,10,2,8,8,7,7,6,4,5,10,9,7,3,8,2,1,1,5,6,8,9,7,6,10,3,1,8,6,10,9,6,6,1,6,9,8,3$, $3,5,1,6,6,7,10,3,2,4,6,2,9,1,6,9,2,7,6,8,9,4,1,2,7,10,3,1,2,2,9,8,8,9,10,7,2,5,10,1,9,3,9,4,3,10,9,2,1,5,8,8,2,10,10,4,7,5,6,5,1$ $0,6,3,5,6,8,9,3,9,4,10,9,8,7,10,10,5,2,2,1,7,1,1,7,10,1,10,9,9,2,9,8,5,4,3,3,7,10,2,5,5,7,5,6,4,5,4,7,5,5,8,4,2,9,10,5,2,10,5,3$, $2,6,2,9,8,8,4,2,7,3,6,6,1,5,3,10,4,2,6,3,7$

$19,7,6,7,9,5,5,8,10,8,1,5,7,10,8,2,1,7,6,2,7,8,4,1,2,2,1,6,9,2,7,10,9,9,7,7,6,3,10,5,2,10,1,8,9,2,10,4,3,8,7,3,9,8,10,9,1,7,5,3$, $3,6,4,8,6,1,1,5,3,8,6,7,1,10,9,1,2,9,4,7,10,2,7,4,3,4,9,6,3,8,2,4,1,3,3,9,5,10,2,5,6,2,10,7,1,5,5,6,7,8,8,8,2,1,4,8,3,3,2,2,10,8$, $1,6,7,9,2,1,9,9,6,2,1,5,5,8,2,10,3,9,4,2,7,10,5,4,9,6,3,9,10,7,2,4,1,8,6,10,2,1,5,3,2,2,10,3,10,8,2,2,6,4,6,3,5,9,3,8,4,4,5,2,8,6$ $, 1,1,1,8$,

$13,7,2,8,4,10,3,10,6,9,10,8,8,8,5,4,1,9,2,8,3,9,9,10,8,9,4,1,7,3,6,5,5,7,10,2,3,3,6,5,1,10,7,8,7,5,1,10,8,3,5,2,4,3,10,1,3,2,2,5$ , 10,8,10,4,7,6,7,1,3,9,5,10,6,2,3,3,3,5,1,7,2,8,6,8,10,8,8,5,4,6,5,2,1,4,2,2,3,2,8,8,7,7,2,5,7,9,2,1,5,3,1,4,6,10,2,2,2,10,3,10,1 , $1,5,5,1,3,10,10,6,3,4,8,8,9,1,4,7,2,4,1,3,3$,

$14,3,6,5,7,7,8,4,9,8,3,4,6,6,10,3,4,7,8,5,1,10,3,9,8,2,9,1,2,3,4,5,6,9,3,2,2,3,4,10,10,4,1,5,3,4,7,10,9,4,6,4,4,7,5,8,2,1,8,8,10$, $2,3,9,9,6,3,7,3,4,1,3,9,8,9,5,8,10,10,1,10,3,10,4,1,6,8,7,8,4,10,1,8,7,3,10,9,6,8,5,9,9,8,3,6,7,5,10,1,8,8,9,9,10,6,3,4,4,3,1,10$ , 2,8,6,2,9,6,5,2,8,10,3,2,4,6,10,7,1,9,7,8,6,10,8,3,5,1,6,6,1,8,5,2,2,5,10,1,3,1,8,1,2,7,9,1,7,10,7,6,3,8,2,2,1,3,5,5,1,7,5,4,5,3, $10,8,3,7,8,3$,

$17,8,7,10,6,10,10,2,1,9,9,5,5,4,4,2,2,2,9,4,3,5,5,10,7,6,9,7,10,1,4,8,2,9,2,2,1,9,6,1,9,9,7,4,8,3,10,1,4,5,3,9,2,3,5,5,3,8,2,2,4$, $10,6,7,9,1,1,10,8,8,7,6,5,9,10,4,6,9,9,7,7,10,5,5,3,4,7,8,5,3,4,1,3,6,10,9,10,10,5,8,7,4,8,1,1,7,6,10,9,3,4,4,2,6,10,4,3,1,4,9,3$ , 7,6,10,2,2,8,3,4,6,7,1,5,7,9,2,6,8,8,6,4,1,4,7,5,5,9,4,3,3,1,10,6,10,2,9,7,2,8,3,10,8,1,1,9,1,6,7,5,4,3,5,4,3,7,6,1,3,7,7,3,8,7,1 ,7,10,9,4,3,2,2,7,4,2,2,3,8,10,3,6,8,10,4,3,5,8,10,4,7,2,2,10,8,3,3,4,8,5,2,9,8,8,1,1,1,6,1,7,9,6,7,4,5,3,8,6,6,7,4,1,9,3, $14,3,7,10,9,10,8,4,7,9,1,2,4,4,10,7,4,1,5,6,2,5,7,9,3,2,10,10,8,7,7,6,4,10,2,4,9,7,1,5,5,7,2,2,3,4,8,6,2,4,3,8,7,4,1,9,9,5,5,2,9$, $7,6,2,8,8,4,9,2,6,8,3,4,10,6,4,3,5,8,3,7,3,6,2,9,8,2,8,7,2,10,5,5,2,1,2,2,2,6,8,3,3,2,9,2,1,10,2,7,6,1,6,6,7,5,4,1,9,4,2,7,3,8,5,2$ , $7,1,10,6,3,9,7,7,10,5,10,4,8,8,6,8,6,3,1,8,8,7,9,10,2,10,5,4,3,7,7,5$,

$20,4,7,9,6,1,10,2,9,1,4,7,8,10,4,4,7,8,8,7,2,9,9,2,6,4,1,7,8,6,5,9,7,9,8,3,9,7,4,4,2,6,6,2,10,5,6,10,3,9,3,6,8,9,2,10,5,2,7,8,6,9$ , 4,2,5,6,4,3,2,5,8,7,9,8,3,9,4,5,7,5,3,3,2,5,6,4,9,3,5,6,8,2,10,9,4,5,2,9,8,10,8,7,5,9,10,4,5,1,8,10,2,10,6,2,1,1,9,3,7,8,4,1,5,3, $6,10,2,9,1,2,8,6,7,4,9,4,10,4,3,5,2,10,2,6,6,8,9,4,1,7,9,7,8,5,7,8,2,1,2,8,3,9,3,4,9,9,4,10,8,10,7,1,10,6,6,7,5,1,2,4,5,8,10,3,8$, $5,2,2,3,1,5,8,7,6,9,4,3,1,5,2,6,10,2,4,10,8,4,4,1,5,4,8,8,6,9,7,4,8,10,4,1,6,2,10,5,10,7,5,6,5,1,10,10,2,8,7,2,6,10,8,3$,

$11,1,9,8,1,9,4,5,9,6,1,7,4,5,7,5,10,6,8,2,6,8,5,4,5,6,4,7,10,10,6,1,7,3,8,1,10,6,5,7,8,5,3,4,9,9,10,6,9,10,4,6,9,10,3,9,7,4,6,9$, $1,5,8,9,2,9,10,2,5,2,10,8,7,7,5,10,3,9,3,1,10,5,10,4,6,3,4,2,7,6,6,8,8,9,5,7,6,9,3,6,7,3,9,3,1,4,10,10,7,7,5,6,6,5,8,4,6,10,5,1$, $10,8,3,3,6,1,1,3,9,2$,

$12,2,3,6,1,1,8,4,2,7,8,8,5,6,1,1,6,9,4,10,8,3,9,8,1,1,9,9,6,5,7,1,8,5,5,3,4,10,3,5,9,8,5,9,5,5,4,3,1,1,6,10,2,4,9,7,3,2,8,8,2,2,8$, $3,9,9,1,2,6,5,5,8,4,6,3,7,7,8,4,10,3,2,2,7,5,9,3,4,4,6,1,10,9,5,6,5,8,8,10,7,7,7,5,3,4,2,3,5,2,9,1,9,9,1,4,9,1,6,9,8,3,7,10,3,8,4$, $4,10,3,2,4,3,8,3,5,2,2,2,5,5,4,2,8,10,2,9,8,7,1,4,6,1,4,8,1,10,7,2,5,9,8,4,6,10,2,7,1,5,3$,

$12,7,2,4,9,6,4,8,7,10,6,6,3,10,5,5,4,3,7,10,8,2,2,6,2,2,4,8,1,6,2,10,2,7,1,4,1,3,4,7,2,9,7,5,5,5,10,9,7,3,7,2,4,8,3,8,8,9,9,5,5,2$ , 7,9,10,10,4,2,2,2,3,6,10,4,9,9,1,8,5,2,6,7,4,6,9,5,8,3,8,1,1,10,6,4,6,1,9,6,3,10,7,3,7,1,2,2,9,3,8,4,2,5,10,9,3,7,2,3,4,6,9,5,6, $4,7,7,8,6,10,1,1,4,9,10,4,2,7,3,8$

$15,6,7,3,3,8,8,2,5,5,10,3,4,10,3,5,4,7,7,1,8,10,8,3,7,9,9,6,10,1,6,10,4,4,5,3,3,5,2,9,1,9,1,2,10,6,5,7,2,2,6,5,10,5,8,10,4,9,7,4$ , $1,5,5,8,3,3,3,2,3,1,8,10,9,6,6,3,9,3,3,10,7,6,2,4,5,9,6,1,10,8,4,3,9,2,5,6,4,9,6,7,4,2,6,4,5,3,10,10,1,10,7,1,3,5,6,4,8,10,3,8,6$

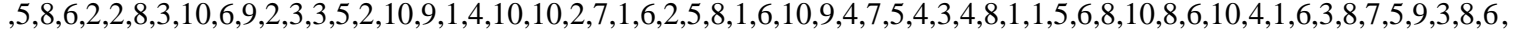
$15,7,2,7,1,2,8,8,7,1,10,5,5,2,9,10,5,5,6,10,8,8,3,2,4,4,5,4,7,6,4,1,8,7,2,9,4,6,6,5,1,7,3,1,10,4,6,3,9,4,8,3,3,9,8,3,7,1,6,8,6,4$, $6,10,4,6,6,2,10,7,1,2,5,7,9,10,3,5,4,2,2,1,10,5,1,10,2,6,10,2,3,6,8,2,8,10,8,2,8,5,7,7,6,4,10,9,9,3,9,1,2,5,4,7,5,5,3,3,7,3,9,4$, $10,3,3,5,6,8,4,9,1,4,8,1,3,10,10,6,9,2,8,7,4,3,7,4,8,9,1,5,6,9,8,6,8,8,4,3,10,5,7,10,8,6,5,6,9,1,10,6,3,3,6,4,8,7$,

$18,3,1,1,5,7,8,8,2,7,1,8,2,10,10,6,4,10,1,10,6,10,2,9,5,3,7,8,8,9,3,9,9,6,5,6,2,9,4,8,10,1,6,5,3,2,9,8,8,10,4,3,7,7,3,5,6,1,4,3$, $8,4,9,4,10,5,7,4,10,8,6,9,5,7,6,6,4,5,9,2,6,3,5,10,10,3,8,1,9,7,3,4,9,5,4,6,8,9,4,2,7,10,5,3,10,1,5,1,4,2,7,6,10,7,10,9,5,3,5,10$ ,3,5,8,2,7,9,10,7,5,1,3,2,9,4,6,2,2,3,4,2,8,6,1,10,8,4,2,6,1,10,2,7,4,8,5,3,4,9,2,5,10,9,4,2,8,1,9,10,5,8,3,9,7,1,6,7,10,8,2,10,9 ,5,2,8,3,10,7,2,10,6,4,3,1,4,2,7,10,9,3,4,9,1,7,10,1,5,4,1,5,4,7,10,1,8,2,9,2,5,5,

$11,3,3,5,9,1,1,3,8,2,9,1,7,8,9,4,2,6,8,5,9,10,10,3,8,3,4,1,3,4,8,2,1,5,4,7,1,9,10,7,6,4,2,10,4,5,5,1,7,10,7,3,10,7,9,1,5,2,2,4,1$ $0,8,6,5,1,1,4,5,8,8,9,1,4,6,9,5,8,7,10,9,9,10,1,3,1,7,8,10,6,9,10,5,1,4,4,5,7,4,5,7,1,8,10,7,5,7,1,6,6,3,9,7,10,2,4,1,7,9$, $20,6,9,3,1,4,4,10,8,2,2,2,10,9,5,2,1,9,1,8,7,5,8,10,6,1,2,5,1,1,2,4,4,3,5,3,1,8,2,6,4,6,1,9,1,1,9,4,5,2,8,9,2,4,10,2,9,9,10,1,6,4$ , $2,10,1,5,1,3,2,6,9,8,10,7,2,10,5,1,1,7,8,5,9,9,4,7,2,9,3,5,7,7,10,6,6,6,6,7,2,10,3,2,3,9,1,6,8,4,1,10,7,7,9,7,8,10,5,9,6,7,10,1$ $0,3,10,1,7,4,3,2,2,6,8,8,9,3,4,4,2,1,10,8,7,8,10,3,3,9,9,5,6,4,2,2,6,7,1,1,3,8,9,10,5,6,4,1,5,1,6,5,10,10,5,9,9,2,4,3,4,4,10,7,9$, $4,3,7,4,10,5,6,6,6,7,9,8,6,8,8,9,2,4,4,2,1,6,7,7,5,3,3,3,9,6,5,6,1,2,8,8,7,10,7,3,5,7,8,1,2,6,5,6,7,10,6,1,3,10,10,4,7,10,10,4,5$, $7,4,1,9,6,6,3,5,5,3$,

20,5,3,5,8,1,6,1,4,4,10,8,4,9,4,4,1,1,6,3,2,6,8,6,3,1,6,8,4,4,1,6,5,1,1,5,1,5,7,10,6,7,5,4,9,3,10,5,5,9,4,6,8,2,4,4,2,10,2,9,3,8, $9,1,4,8,6,8,2,7,5,8,1,6,10,8,8,9,8,2,10,5,8,8,6,10,8,6,3,1,10,7,7,4,4,9,2,4,8,6,9,8,4,4,10,1,5,6,1,2,3,1,6,5,5,10,8,3,4,8,10,5,4$, $6,6,10,6,8,10,4,2,5,1,6,3,4,5,1,4,1,9,4,8,10,7,8,3,10,7,2,7,5,6,6,9,5,8,10,4,3,10,1,6,6,3,3,8,5,5,9,5,3,4,8,8,7,7,2,8,4,6,10,5,9$, $10,4,5,2,2,8,7,7,9,4,3,6,8,5,4,9,1,3,9,7,9,10,1,5,2,1,1,4,1,3,2,2,9,8,3,9,8,1,2,1,9,2,8,9,6,4,4,7,10,10,5,6,6,3,7,5,1,1,10,7,10,1$ $0,5,7,1,6,9,8,4,1,8,2,6,1,3,5,3,9,4,1,6$, 
$15,8,2,5,1,10,9,6,8,10,3,2,5,4,10,7,4,9,1,9,1,2,7,10,4,5,2,7,4,1,2,9,6,5,3,6,10,8,9,4,8,6,7,3,4,10,5,1,1,5,8,8,1,9,3,5,1,2,1,6,2$, $7,7,4,5,3,7,7,7,7,9,3,8,6,3,5,5,8,6,6,10,9,2,4,7,2,9,5,2,10,3,10,1,10,10,6,9,2,8,8,8,4,10,6,1,7,1,9,10,5,5,10,9,3,10,6,5,7,10,6$, $6,10,7,7,9,8,2,7,4,9,1,10,8,5,8,6,9,7,5,9,9,4,10,9,1,4,3,10,8,10,4,5,2,10,4,7,4,5,4,9,2,6,10,3,1,10,8,8$,

$18,2,4,2,10,6,10,5,5,9,7,8,5,6,9,3,8,10,10,1,7,2,5,4,3,7,1,2,8,5,4,9,5,2,6,3,1,10,9,9,2,6,6,8,10,4,4,4,8,8,7,8,3,1,2,9,6,9,9,3,5$, $1,6,8,2,3,2,4,8,6,10,5,2,8,9,7,3,4,10,3,4,2,1,3,8,8,2,9,7,1,4,3,10,6,8,3,6,9,3,10,9,8,2,6,6,2,1,3,2,10,9,8,5,10,8,7,9,4,6,10,1,9$, $6,6,7,5,4,1,4,3,1,4,7,3,1,2,3,7,8,6,4,2,2,4,10,1,10,7,10,9,4,7,2,6,7,5,9,9,6,10,3,7,5,1,10,8,8,9,2,4,6,1,1,9,7,4,9,5,6,9,1,4,10,9$ , 5, 7,4,8,3,8,2,10,8,2,6,4,5,8,4,7,5,1,3,3,6,5,7,9,7,

$10,10,5,1,8,8,4,6,3,1,6,2,2,10,7,9,10,3,1,10,9,1,2,3,1,5,6,6,4,6,9,2,2,2,1,1,10,10,5,8,1,10,3,5,9,1,4,3,6,10,1,9,7,7,1,5,4,1,8,4$ $, 8,4,7,10,8,8,7,1,5,6,1,5,9,7,3,2,10,2,10,4,9,6,3,6,2,8,5,1,8$,

$17,3,5,5,9,4,4,2,10,9,10,2,5,5,6,10,4,4,6,7,9,8,1,6,8,3,5,1,2,9,3,8,5,3,6,6,8,2,4,7,10,1,1,2,7,9,2,4,6,6,6,5,2,8,6,7,6,10,2,2,3,6$

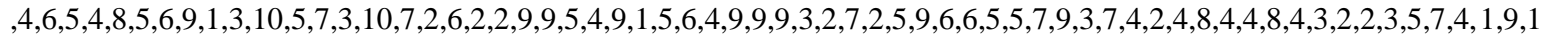
$0,1,3,4,8,6,5,8,1,5,4,9,9,1,8,7,3,2,9,1,4,10,9,10,9,9,1,3,10,7,7,6,5,8,2,5,6,4,7,2,3,10,9,6,8,10,5,4,4,1,2,2,3,8,7,7,10,8,6,4,1,8$ , $1,2,5,8,9,4,1,1,3,1,8,8,6,10,4,3,2,2,7,7,1,5,9$,

\section{Instance 8:}

$20,15,2$,

$20,1,11,4,12,10,9,5,8,8,5,13,1,1,6,3,8,15,2,4,6,7,4,6,10,14,2,11,9,6,7,2,10,1,8,3,3,8,12,3,5,6,12,3,9,2,9,1,6,11,8,15,7,12,8,9$ , $2,14,1,7,7,13,4,5,4,6,5,3,8,8,12,5,1,2,11,11,1,2,5,14,2,1,4,6,4,8,7,15,5,10,1,9,8,12,9,5,7,4,15,7,3,4,4,9,12,3,14,1,1,7,4,5,2$, $12,6,11,2,10,7,3,6,9,5,14,7,8,7,2,3,4,9,6,5,13,3,15,3,10,10,8,2,3,12,3,15,6,7,3,8,3,11,3,5,7,14,2,6,3,4,7,9,9,1,5,13,3,3,1,4,5$, $1,14,5,9,7,7,12,4,13,5,2,4,11,8,10,4,3,10,4,7,15,5,2,10,9,6,8,14,13,3,8,1,7,9,1,7,11,7,15,1,4,8,2,1,6,5,14,5,5,4,10,2,3,4,12,1$ $0,3,5,10,13,7,4,10,6,12,1,11,3,7,8,4,2,14,9,13,7,12,2,7,12,8,6,4,13,5,9,1,11,4,15,8,5,8,8,3,3,4,1,9,14,1,12,12,9,13,2,15,9,14$ $, 8,11,2,3,7,9,4,8,6,6,3,10,6,5,2,4,6,9,8,5,9,10,7,7,5,10,11,2,13,6,1,10,2,5,3,8,2,3,9,8,5,2,8,4,7,1$,

$17,6,4,7,5,10,15,10,7,6,8,1,2,2,9,11,1,13,9,15,8,3,2,10,10,5,8,9,8,4,5,6,10,2,9,5,7,6,5,9,5,7,4,15,2,1,6,11,9,10,1,6,14,2,11,2$ ,12,9,15,9,3,8,9,4,13,3,7,1,4,9,14,2,5,10,6,13,5,8,7,5,10,15,4,7,7,14,5,11,1,1,2,12,2,4,8,3,8,6,7,13,11,4,8,8,12,8,3,6,10,9,9, $8,7,10,14,7,4,7,6,4,15,3,5,5,13,6,4,8,7,12,2,15,2,3,5,5,8,5,6,5,3,10,12,8,9,9,14,1,1,8,2,12,5,9,8,11,9,13,4,3,8,5,1,15,4,10,3$, $6,3,4,9,7,3,2,6,13,3,10,6,5,4,6,12,7,11,7,7,8,9,1,8,3,15,4,13,1,2,5,14,7,5,1,5,3,3,10,10,8,8,7,4,6,7,13,2,10,15,9,3,1,10,2,5,7$, $7,2,14,10,6,6,8,3,4,10,13,1,12,3,9,3,8,13,2,7,6,9,10,6,9,12,3,3,6,1,1,5,9,13,11,2,2,1,7,6,12,6,9,6,13,7,3,2,10,5,14,8,5,6,4,3$, $15,7,6,3,15,11,1,5,1,15,5,4,9,14,5,6,2,9,5,10,3,8,4,13,6,1,6,7,4,3,3,2,4,12,3,13,2,9,14,10,12,3,15,7,10,5,11,4,1,6,8,3,13,5,6$, $1,5,9,4,3,9,7$,

$15,6,5,9,14,6,10,1,6,7,11,1,12,4,3,11,8,10,10,13,5,5,12,6,2,9,7,1,10,10,11,3,7,9,3,6,8,14,9,7,6,3,3,1,7,15,7,3,10,10,11,2,4,5$ , $14,8,1,14,8,15,3,9,7,6,1,11,8,5,3,12,5,7,3,2,8,10,4,4,4,13,4,3,4,10,6,7,8,5,11,1,12,5,10,1,2,1,1,8,13,6,9,4,5,3,15,15,10,10,1$ , $12,8,9,4,13,4,3,3,5,1,8,6,6,10,2,9,11,2,14,8,4,9,1,5,7,10,5,9,5,3,2,13,3,1,10,8,4,11,9,8,14,6,3,1,12,10,8,8,2,5,6,10,15,9,10$, $6,1,9,5,2,14,9,7,4,9,6,4,2,2,7,4,8,5,14,1,13,1,5,8,11,4,3,3,10,7,15,5,1,5,5,3,3,14,1,7,4,5,10,10,3,8,8,9,13,6,12,7,4,8,11,10,9$, $6,10,8,15,5,1,8,9,10,2,9,12,6,3,3,6,5,5,5,11,8,9,2,15,9,7,7,10,9$,

$17,14,15,8,13,4,8,8,7,1,1,7,10,3,9,3,11,6,4,4,12,7,5,5,14,6,6,5,3,7,8,3,10,13,6,5,4,10,4,14,9,6,2,11,7,15,8,2,1,2,7,5,15,9,10$, $6,2,3,8,15,8,14,2,2,8,7,4,12,9,4,2,13,10,8,6,11,10,5,4,10,9,1,8,12,7,7,10,10,5,7,12,3,11,5,6,5,9,6,8,3,13,1,15,2,3,9,4,9,5,13$, $6,8,5,4,5,15,10,11,10,8,10,5,4,8,15,2,12,5,11,1,6,9,1,2,9,2,2,5,6,8,7,7,7,4,4,2,1,10,13,8,11,9,8,8,9,2,7,1,3,10,3,13,1,7,9,5,8$, $2,8,14,2,6,3,10,9,10,10,3,1,10,2,8,7,6,2,15,1,10,9,5,3,4,14,8,5,4,6,8,13,2,5,9,9,15,8,4,10,7,1,11,9,15,12,3,1,7,14,9,7,1,13,4$, $2,6,15,10,3,10,9,2,11,1,5,10,10,9,4,5,6,3,8,4,1,2,7,15,1,9,7,3,5,1,12,8,4,7,15,10,6,5,13,3,9,4,11,5,8,6,14,8,3,10,2,6,10,6$, $15,3,7,2,14,2,11,4,2,15,1,14,9,6,15,9,14,10,7,10,9,10,10,8,5,5,4,7,6,14,8,13,5,6,3,12,1,6,5,7,8,2,10,10,14,5,9,6,13,5,11,9,1$ $2,8,7,2,3,2,6,6,5,8,3,4,8,7,2,1,8,11,6,12,10,6,12,6,13,6,14,1,11,2,1,7,8,4,4,1,7,6,6,8,5,7,2,1,10,1,3,15,6,13,4,10,1,7,9,8,7,6$, $6,4,10,12,8,3,5,9,12,1,14,1,15,4,2,6,11,6,4,5,1,7,9,9,7,7,7,8,8,5,10,13,3,11,4,2,8,15,4,9,4,11,7,10,14,8,5,4,9,8,15,2,11,3,6,1$ $0,12,8,3,2,1,2,10,9,4,5,1,14,1,10,5,12,8,7,10,4,14,8,5,4,12,6,13,6,15,4,9,5,5,3,3,15,4,1,5,12,7,7,6,3,15,1,14,10,12,2$, $20,1,13,10,3,9,8,6,10,13,2,7,15,5,9,5,7,1,8,3,12,7,2,9,1,9,3,2,9,3,8,7,10,9,5,1,14,4,8,5,10,4,11,4,15,1,7,3,3,6,12,4,8,2,3,10$, $3,13,7,9,5,8,3,12,8,7,7,1,4,9,5,8,14,9,4,6,12,7,11,1,10,7,6,9,7,5,3,9,8,7,6,9,8,5,4,2,9,1,8,12,3,15,7,8,9,13,11,4,2,3,13,7,7,1$, $10,3,8,5,3,6,1,4,4,6,14,7,12,2,15,7,5,6,1,12,10,14,3,10,4,3,15,5,13,7,6,6,7,1,11,8,8,5,10,7,12,8,5,4,14,5,1,1,2,8,8,12,6,9,3,2$ ,10,3,10,10,8,13,9,5,7,14,5,10,6,8,10,10,15,1,4,9,11,4,1,5,9,8,14,7,7,10,8,10,15,9,1,14,5,7,7,12,10,13,3,10,5,6,10,8,1,15,8, $2,1,4,8,3,9,5,4,11,9,1,3,14,10,5,5,8,15,6,7,1,12,3,2,7,9,7,8,1,1,8,11,2,13,6,3,6,6,1,14,10,10,9,10,14,10,13,3,11,6,8,10,6,5,5$, $6,15,7,7,10,3,7,1,12,6,3,4,5,10,4,13,6,11,4,2,10,9,3,1,12,1,15,3,14,6,9,3,2,8,13,5,6,5,7,3,12,5,9,10,1,13,4,6,1,14,2,15,3,3,3$, $2,8,12,7,7,4,9,5,8,1$,

$17,6,1,8,4,9,13,2,12,2,9,10,5,8,2,13,7,10,8,8,14,6,12,3,13,1,4,8,9,2,10,3,5,7,2,7,2,15,5,14,2,14,15,10,14,3,13,1,2,1,3,9,4,5$, $6,2,12,6,7,3,1,5,9,2,8,6,5,9,11,6,5,15,9,3,5,4,5,12,9,10,9,13,3,5,14,9,1,3,12,2,2,5,11,7,13,6,8,4,7,3,9,6,6,10,4,4,5,4,13,9,7,1$ $4,1,3,2,11,3,6,10,15,5,12,7,10,8,2,6,13,7,5,8,4,1,1,7,3,5,3,10,7,6,1,14,8,3,2,8,6,8,4,3,11,1,10,4,14,5,9,6,1,5,15,1,5,4,7,10,3$, $9,13,10,1,15,8,9,3,3,15,7,1,4,9,7,7,6,13,3,8,9,5,6,14,2,11,14,5,15,4,13,8,9,8,7,4,10,9,11,10,6,4,8,10,4,8,12,2,2,5,3,15,5,11$, $5,2,10,4,12,3,9,7,13,10,8,6,14,7,4,7,7,8,2,5,1,9,7,1,6,8,10,4,9,15,10,11,1,3,5,12,6,14,6,8,14,8,8,2,9,10,5,8,10,7,4,10,11,1,1$ $2,7,2,7,1,4,7,1,15,2,13,10$,

$18,11,1,6,13,5,7,1,12,4,11,4,9,4,15,1,6,3,14,3,8,5,5,2,1,5,1,12,2,1,8,6,15,5,1,2,11,4,10,1,4,4,7,10,12,7,14,4,9,2,13,5,7,11,2$, $1,8,10,2,15,4,5,7,7,5,9,5,10,1,1,4,2,14,9,15,1,11,9,3,9,6,5,13,5,8,7,7,6,12,7,7,14,1,11,6,9,3,1,1,15,10,13,5,8,1,12,3,5,1,10,5$ , 4,6,7,11,8,13,8,2,9,7,4,1,2,6,1,9,8,6,3,1,13,7,1,3,2,1,5,8,4,3,9,13,10,10,5,14,5,12,8,11,7,5,3,9,3,3,6,1,9,15,15,7,14,7,5,8,8, $10,9,6,2,8,3,6,6,6,10,3,4,8,7,7,13,4,12,3,11,6,1,9,1,2,10,11,6,9,8,8,14,5,12,6,9,3,7,10,11,9,3,5,1,7,13,8,10,1,14,10,5,14,6,5$, $3,9,2,1,5,11,6,13,2,3,4,6,8,15,4,4,5,8,7,12,2,2,5,15,14,8,2,5,1,1,8,2,5,7,4,2,6,9,7,4,11,2,13,5,15,10,12,8,3,9,9,1,10,3,13,2,2$, 
$15,2,8,10,3,4,5,7,6,9,7,7,13,10,11,9,14,5,12,10,4,7,9,3,1,6,3,14,14,10,15,7,1,3,2,10,10,8,6,1,12,3,9,4,4,8,13,9,11,4,7,1,3,7$, $5,3,15,11,2,1,1,10,8,5,1,12,1,14,1,4,3,8,7,7,2,13,4,3,6,2,2,9,4,6,8,15,4$, $19,7,1,5,14,9,11,3,4,9,7,5,5,8,9,4,6,11,5,12,3,7,1,14,8,9,4,5,4,6,8,7,5,9,2,9,6,7,12,2,13,10,15,2,9,11,1,4,6,14,3,9,3,8,3,5,6,1$ $2,2,7,7,13,3,10,9,3,10,6,10,1,6,15,4,13,6,7,7,5,12,8,11,5,3,10,9,2,14,6,4,2,2,1,13,2,5,9,15,2,10,8,2,14,7,12,7,10,6,1,5,2,12$, $5,10,5,13,8,2,6,15,3,3,7,14,9,8,8,5,13,10,10,2,1,6,12,6,5,4,7,12,7,14,7,2,7,13,8,15,6,3,10,1,3,10,9,2,2,2,3,4,12,6,15,6,14,10$ , $5,8,10,5,11,8,13,8,5,1,8,2,9,13,2,12,8,11,10,10,11,6,9,6,6,7,7,4,3,8,14,1,13,8,10,8,12,10,4,3,15,7,2,15,7,1,2,3,6,5,5,8,6,9,6$ , $2,6,4,3,13,9,10,9,11,8,6,2,14,5,12,9,14,2,9,4,1,7,4,11,6,14,3,13,3,9,4,8,10,15,10,10,1,6,7,5,3,12,1,3,5,15,12,4,1,9,6,2,13,5$, $2,1,4,2,10,5,15,8,14,4,11,2,5,1,9,2,3,6,8,1,7,4,1,1,1,10,1,8,15,5,4,6,13,3,11,3,9,1,14,10,5,1,12,8,6,4,2,4,3,10,3,4,7,7,15,7,1$ $3,6,2,3$,

$16,10,3,9,14,10,10,9,9,2,4,3,2,4,13,3,8,10,12,3,6,6,6,1,1,4,6,3,7,12,3,8,9,2,3,8,11,10,8,8,5,6,12,8,15,8,10,9,2,10,9,5,12,2,2$, $3,3,1,3,12,3,9,6,4,8,5,1,15,2,13,8,8,8,6,3,11,1,2,5,5,13,8,2,1,10,3,6,15,8,8,1,2,5,10,12,2,10,9,4,5,11,9,14,7,13,2,7,7,6,2,3,2$, $15,4,2,6,9,7,13,2,3,4,9,8,4,6,2,9,6,3,4,12,7,7,6,14,10,5,7,11,10,10,4,1,7,7,5,7,14,2,12,9,10,1,1,10,9,8,6,2,7,12,7,5,1,1,4,8,2$, $15,10,7,1,10,9,8,11,7,14,8,15,10,7,6,3,4,10,8,12,9,1,10,11,7,8,6,10,14,4,11,3,9,2,10,5,15,2,13,10,2,1,8,2,5,4,3,1,6,6,5,4,8,1$ $5,6,8,14,10,5,5,13,1,8,6,4,1,3,6,12,7,9,6,7,7,1,8,10,7,11,9,15,10,2,1,8,4,2,15,8,10,8,2,4,11,3,1,3,13,7,3,7,3,7,3,9,3,15,3$, $20,4,5,9,8,9,6,4,4,3,14,11,4,15,10,9,2,13,5,3,6,10,2,2,6,7,3,8,1,6,3,4,10,5,5,14,4,12,4,1,13,3,10,5,5,14,6,4,3,10,8,1,5,11,5,9$ , 4,2, $2,6,6,8,5,11,11,7,12,10,8,4,13,2,15,10,6,9,9,4,10,3,5,8,14,3,4,7,7,13,7,6,9,15,2,5,10,2,9,10,5,9,7,5,9,5,14,8,5,4,7,2,4,2$, $7,2,3,1,7,5,10,12,9,15,8,8,5,9,10,2,7,8,9,8,14,2,8,1,5,5,1,12,10,4,7,10,3,11,8,6,6,13,8,7,6,15,6,9,7,14,10,8,7,3,15,10,1,8,11$, $3,13,8,3,1,5,3,5,15,9,12,5,4,8,13,1,2,4,14,6,7,2,9,7,5,9,6,4,2,1,5,5,8,8,11,1,7,3,3,6,8,5,14,8,15,8,5,7,13,7,4,9,4,1,10,8,4,6,8$, $1,2,7,7,8,7,14,6,13,6,6,6,1,7,12,7,9,5,1,4,5,15,10,5,14,5,13,7,9,5,6,5,11,10,12,2,1,7,5,9,3,6,15,2,4,10,7,9,8,6,2,8,12,10,10,6$ $, 8,3,5,2,2,11,5,8,7,13,2,12,7,7,9,9,7,5,4,4,10$,

$19,3,4,5,5,5,12,10,7,6,2,7,8,13,4,5,2,12,1,1,7,11,9,13,5,3,9,10,14,2,8,1,15,7,4,3,10,2,12,9,2,2,3,4,7,4,13,9,6,9,12,5,4,4,4,12$ ,3,14,1,1,3,2,5,9,8,13,1,6,2,7,4,11,9,3,2,3,5,9,2,8,8,6,15,3,5,6,7,15,4,9,6,1,2,14,8,11,4,13,5,7,5,8,10,5,2,4,10,10,2,12,1,2,2, $6,9,9,14,1,7,7,15,9,11,8,3,7,11,8,1,1,6,13,8,12,1,3,4,15,10,11,1,2,2,6,5,7,9,10,8,14,10,1,7,3,13,6,9,6,5,5,1,1,11,8,14,8,15,1$, $4,7,6,1,12,1,3,6,8,1,6,15,9,8,4,2,6,6,8,4,8,10,9,7,7,6,5,8,3,9,14,7,13,2,11,3,6,4,13,6,2,13,3,8,2,5,4,11,3,10,4,1,8,3,8,2,4,15$, $2,9,8,12,9,14,5,7,10,8,8,6,1,3,6,6,11,7,2,8,7,10,5,3,4,15,7,4,5,12,7,5,6,12,15,8,11,8,5,8,2,8,6,5,14,9,3,10,8,2,7,8,10,3,4,2,9$, $5,8,6,6,14,8,13,9,15,6,11,10,9,8,7,1,10,9,12,9,6,15,4,1,8,12,7,11,10,7,10,14,3,10,4,5,10,6,2,8,8,13,7,5,6,10,10,6,12,9,15,8$, $1,9,13,7,9,13,5,4,3,9,1,6,4,10,6,12,9,8,9,15,7,11,2,14,4,3,9,5,4$,

$17,13,3,5,14,3,5,9,9,9,1,2,7,6,6,6,15,10,13,1,8,7,12,4,4,4,11,3,3,2,6,12,4,13,1,3,1,10,14,6,12,5,5,1,5,15,1,7,9,5,6,10,7,4,9,3$ $, 11,7,14,8,12,1,13,5,7,7,5,15,2,6,4,2,10,10,7,12,9,8,1,4,1,9,3,14,4,3,4,13,4,3,8,6,14,6,6,2,6,2,1,1,6,10,7,12,4,11,4,9,10,12,7$ ,4 $14,3,15,2,12,1,11,8,8,4,9,4,4,10,10,9,1,7,2,10,3,5,13,12,8,9,4,1,1,2,2,8,1,3,1,15,3,6,10,14,10,5,10,10,5,4,3,13,5,15,10,9$, $11,7,12,1,14,1,4,7,9,3,7,5,1,10,15,10,13,6,2,10,6,10,3,6,5,9,8,2,6,4,4,9,2,2,8,15,1,5,7,14,6,10,11,9,6,1,9,3,3,6,2,10,10,7,7,9$ $, 1,3,13,3,14,2,13,12,10,8,9,11,3,10,9,7,3,5,6,9,8,6,7,14,1,2,5,15,4,4,1,13,5,10,6,4,14,1,7,2,2,2,5,3,15,3,4,3,13,6,9,9,10,2,11$ ,14,1,12,9,8,7,10,2,11,9,1,10,4,10,9,4,15,6,6,8,5,10,13,3,10,11,4,5,8,2,9,14,6,8,4,9,1,6,7,7,6,13,3,4,3,10,8,12,9,

$19,13,10,4,5,5,13,3,6,10,3,4,1,3,9,6,8,5,12,3,11,5,7,1,4,2,15,3,13,2,6,11,4,14,5,12,7,13,10,10,6,9,2,3,2,7,8,8,5,6,10,4,5,15$, $4,8,1,8,15,3,12,2,10,7,11,10,8,3,3,9,6,10,7,9,2,8,6,3,3,1,4,11,6,10,4,7,6,13,5,10,12,8,2,7,4,1,11,8,14,6,15,10,8,10,7,9,6,4,1$ $0,2,13,5,3,7,11,8,1,14,9,13,6,3,10,11,6,9,7,12,7,15,7,10,10,1,9,6,4,15,6,4,5,7,3,9,7,3,15,4,9,9,14,10,8,6,2,6,12,10,4,3,10,8$, $11,3,1,10,13,2,3,5,1,14,4,3,6,3,7,3,11,3,1,9,9,10,9,14,9,13,5,4,3,7,3,9,10,15,4,12,3,11,8,10,9,4,4,2,15,8,2,9,11,3,14,7,5,2,1$, $4,10,2,8,6,1,4,10,7,1,2,12,3,13,10,9,5,7,8,15,10,3,5,5,5,5,12,4,1,4,2,7,4,7,7,8,4,10,5,13,5,5,1,1,10,14,7,12,10,15,12,5,3,9,1$, $9,14,9,5,6,10,3,6,3,8,3,7,2,4,4,15,4,2,8,11,10,13,8,9,9,2,13,5,10,8,15,12,7,11,10,13,5,1,1,10,7,5,7,4,3,9,2,14,3,7,9,8,10,15$, $4,2,6,3,2,6,4,2,14,9,3,1$,

$17,12,3,6,14,2,4,6,7,1,13,4,15,7,12,9,9,9,6,5,2,3,5,10,10,9,6,3,9,4,2,6,2,5,6,9,3,15,3,9,6,6,4,2,8,7,1,2,11,6,15,10,5,3,7,6,9,8$ ,10,15,5,5,2,13,8,11,8,3,4,6,5,9,2,8,5,12,1,10,8,15,1,4,7,1,12,6,9,7,13,9,2,1,8,1,5,9,3,7,6,8,10,4,4,4,14,7,11,9,15,3,10,7,1,1 $3,3,8,5,5,10,10,10,11,6,9,2,2,10,4,5,12,3,13,8,1,9,2,6,7,15,3,2,4,3,8,7,7,11,3,14,6,12,6,5,9,4,1,13,10,5,10,9,8,6,2,3,11,8,5,7$ , 7,2,3,11,3,14,3,12,5,3,6,5,2,10,6,6,1,5,13,4,10,4,7,2,2,10,5,9,7,6,1,12,6,1,3,15,1,3,1,8,10,10,5,15,7,6,13,2,12,9,3,10,11,5,1 $0,5,8,8,6,9,14,4,15,4,5,7,9,3,4,9,1,5,2,8,3,2,3,6,1,13,3,1,12,1,10,10,6,6,9,13,7,8,3,9,5,15,5,3,8,14,4,2,10,7,6,3,5,2,12,2,14,6$ ,7,1,10,2,10,6,2,5,9,11,1,7,5,12,4,

$19,4,5,5,6,9,8,7,12,8,10,10,2,8,2,4,6,3,8,12,6,5,4,14,4,1,6,6,8,7,5,13,1,9,14,3,15,9,7,4,6,7,2,1,13,8,11,4,10,5,8,5,5,5,12,10$, $3,3,9,1,9,5,9,9,10,15,3,10,8,8,7,4,7,12,7,2,3,2,15,4,3,6,10,2,6,10,5,13,5,15,6,1,8,3,10,9,6,5,3,14,5,6,6,11,9,8,2,7,7,9,12,2,1$ $1,1,8,5,10,6,3,1,15,9,13,3,5,1,8,14,8,5,4,11,4,12,6,1,4,3,2,4,8,6,6,5,6,8,9,9,14,6,2,8,13,5,7,13,10,11,6,7,7,12,10,14,7,3,5,10$ , 7,6,4,4,13,10,7,1,15,6,11,8,3,3,5,4,3,3,5,10,9,9,2,12,1,4,11,8,5,1,14,10,12,3,12,10,9,14,1,12,9,11,7,5,7,3,4,8,3,13,9,2,7,6,9 , $1,5,4,5,14,6,6,3,6,13,4,2,3,11,3,10,1,8,4,15,4,9,5,14,4,5,8,12,1,7,2,1,4,14,15,10,14,6,7,9,9,7,10,10,5,10,12,2,8,3,4,10,11,6$, $6,1,13,4,3,8,2,5,9,15,9,7,7,1,8,2,5,13,1,14,7,9,7,12,8,6,9,2,4,5,1,4,3,7,6,12,6,15,7$,

$19,15,14,10,5,9,15,1,8,3,9,3,10,8,6,3,13,4,7,8,11,1,1,3,12,4,2,7,4,2,3,7,3,15,2,14,8,2,7,13,13,7,6,3,11,6,2,9,15,7,10,1,12,10$ $9,6,3,7,7,4,5,4,14,9,4,9,11,15,10,1,5,13,8,3,10,8,3,11,10,9,1,5,5,12,7,7,6,2,7,2,10,5,12,7,1,2,3,15,2,5,12,7,6,5,14,6,9,9,10$, $9,3,1,15,4,7,10,13,7,8,10,4,1,1,10,5,9,11,4,8,11,6,5,1,14,1,13,3,10,4,4,5,8,6,6,3,6,15,5,11,7,2,4,3,9,9,3,14,1,9,10,2,15,9,4,5$ , 2,2,5,10,8,10,14,9,13,5,7,2,7,15,10,7,5,14,8,12,7,2,2,13,3,1,6,14,1,7,6,4,11,2,12,10,7,10,10,2,8,6,15,4,13,5,14,3,5,8,3,5,4, $10,9,2,5,15,8,10,1,6,2,14,4,11,10,1,14,1,5,12,8,15,4,5,5,11,9,3,5,6,11,6,15,4,5,3,6,1,2,9,12,4,4,14,2,13,8,12,8,1,5,5,1,10,11$ $, 8,13,6,12,7,2,10,5,3,2,14,8,6,7,12,10,2,4$,

$16,11,5,1,14,8,12,8,7,7,1,2,13,9,10,10,8,7,15,6,6,8,3,10,10,12,7,14,9,8,8,15,7,10,5,4,1,9,2,11,7,2,4,7,2,6,3,9,14,2,10,5,4,4$, $5,5,13,9,14,1,1,14,9,9,5,12,6,6,2,11,5,15,2,13,7,5,5,7,10,3,2,8,2,4,1,2,2,15,3,10,11,10,9,1,7,8,14,2,10,9,8,1,6,10,12,3,15,3$, $1,10,4,2,2,1,13,6,5,4,2,11,8,4,8,12,15,1,6,10,1,2,2,8,13,2,12,2,9,5,11,7,7,6,8,3,14,10,4,7,3,1,8,12,1,6,1,12,3,9,11,10,8,3,2,4$ , $9,7,5,6,15,10,13,8,4,3,6,2,12,6,10,6,13,6,7,12,8,14,9,9,10,11,8,10,7,13,1,15,9,3,6,7,3,5,10,1,6,4,9,5,1,1,5,1,15,7,11,5,13,8$, 
$5,12,8,14,8,6,5,9,8,10,3,11,15,7,10,3,4,5,7,8,11,8,3,7,14,4,8,8,9,5,6,1,13,1,13,5,3,14,9,13,8,4,5,3,6,15,8,10,2,1,3,8,7,9,9,6$, $5,7,1,11,5,6,3,5,8,1,13,9,14,5,9,9,12,5,8,15,7,10,2,14,7,3,2,7,6,4,8,11,3,8,9$,

$18,14,14,3,4,8,11,9,12,10,9,8,7,10,8,7,1,8,6,2,13,7,10,7,15,10,3,1,5,10,2,2,7,6,3,9,1,8,4,4,2,1,3,9,14,8,13,4,5,8,7,3,12,2,10$, $2,5,14,3,13,4,12,4,10,5,1,3,9,1,6,1,11,9,5,3,9,3,8,8,5,14,9,1,3,2,4,10,5,11,3,9,2,7,4,6,15,1,13,10,8,3,1,4,9,5,5,10,8,13,1,12$, $1,6,1,2,1,9,7,10,2,5,5,15,5,2,4,8,9,10,12,4,1,5,6,12,1,14,7,9,1,1,10,6,4,8,10,13,3,10,4,3,4,15,7,15,6,2,11,4,15,5,14,7,8,5,5,3$ , $13,6,9,6,4,1,10,8,12,3,2,2,3,5,1,8,7,10,2,3,9,11,10,5,9,9,4,10,1,8,10,4,2,7,9,5,9,9,6,7,9,12,7,11,9,8,1,15,3,13,1,14,4,3,9,7,6$ , $7,2,7,12,12,3,3,4,13,5,14,4,9,9,10,3,15,8,4,1,8,7,2,1,5,5,11,8,1,10,4,12,7,9,13,4,9,4,1,7,8,4,5,9,15,2,11,4,12,4,6,2,2,1,3,3,7$ $, 8,1,4,8,6,7,15,6,13,2,1,9,2,2$,

$17,13,14,9,7,8,4,4,3,3,11,7,10,2,13,3,12,2,1,3,6,6,8,9,2,4,9,3,12,12,1,15,10,8,2,2,6,11,3,10,7,9,6,3,5,14,4,6,3,1,1,4,3,15,2,4$ ,10,2,7,3,12,5,1,1,14,5,11,5,8,8,3,2,9,10,5,9,15,1,13,4,4,7,6,9,7,3,5,9,9,10,6,13,6,15,9,14,7,1,8,4,6,8,1,8,13,7,4,1,9,3,8,7,3, $5,3,6,2,13,2,10,5,15,5,1,1,4,7,1,5,2,11,11,8,14,10,13,3,7,8,8,7,9,2,12,9,6,1,1,1,15,2,4,4,6,12,6,10,10,15,9,3,2,14,10,13,10,7$ ,3,2,2,3,10,5,5,3,11,6,8,4,9,2,2,1,6,12,4,11,4,4,2,10,1,10,12,9,11,5,14,1,15,8,9,5,7,1,6,10,5,6,8,2,5,14,2,15,10,11,9,1,9,10,6 , $5,10,3,9,9,2,1,13,5,1,10,5,7,4,10,10,1,3,2,8,10,12,6,15,10,1,4,7,8,3,6,8,1,1,14,2,9,8,13,5,5,4,3,2,15,5,12,4,11,4,2,10,7,6,9$, $8,8,15,2,14,9,9,10,11,7,10,5,7,9,2,10,4,7,13,4,7,14,9,5,5,6,10,12,3,2,6,9,4,8,8,7,9,15,8,1,7,10,2,11,7$,

\section{8 real instances with fuzzy processing time}

\section{Instance 1:}

542

41256743591111234910142681023468113434246391015181013

5331254123257944123157933562457241341781111791014569

4134574367104479168122581022345189124447101357212536710

51112344145169102579345634569224537910333571791141233356721341679

53113421234356448101436910112326784134621344791333454291011312315784 891212134

\section{Instance 2:}

882

8381236123169116556821346468468107357313451456478126147235757810875 8123123613485674810125134134626913271345479661233246212353454456735 77181014612381235245245649101571238513471236479214517911379124567813 5

82358116479435696910147134512322147791014411358567635755697814646897 13453456345312325811251248681017581136910154910121457

836571079101585812338912257987911244576123241473134233574591255691225 8126134378947893157931348791071671086911347851236710152710124134 8866913847937811457973571134234653466824536911156751234791375678645 78356278115691371343591016794567267101484684624575913445755693179125 78117245815911513423563781165684345781014834576136756832452124812348 10131346

856356771012456951238910157113436710269108681043456457768122212335671 59101566346734622463479845655694771013867101357581012281343246552462 245391011413486910

8311237457413481123312357810412388913656771232679566101121234123824 5113636781181233467586785346656916910467914123882451710116134758941 34378925685356485677471028101366913

8853451456613434710412371342791185693156975676578881243245778114356 26810534666911158117713668101541342467513485912147825123812317457214 5644710735812791015267915912591011413486911

88657971231134558118123256731234134345692123547103161013245748913469 10132567767105571058134512371231591265781713472678546831348691242461 3457681148134713461255123

\section{Instance 3:}

1062

83256731235346656792479324648101165911113424456591011647812512324710 313416710612322678567854791057101131342891111345234665810469121891257 8931910115691137812 
10357893245167825123312365123224549101211343781069101231123256831461 51345234656786479313445694367841341357612325569691011361341346491012 1661014

736469513435910236911659121491015217812634623123212333134571011145624 134691012

816134151345135636782910114910135478266910213442356381014435667910461 234691154573469537101511244134691014547952479545763573125491013

963123679124910112810155345135745457157843562910133247958101441345414 6112326793123657105413528101117896124312315567122462289114569515684 1465145679122456

85413425795910133468613431468345751346614511344691032452910155681126 4695136259101421346668125145457812463891325684624654681145413461134 5610113134412322466357

65556734784467178106689411233467213457101311134437810458125457257826 68912466245613455245324547896569

853810154123112358911281013216910567911357231346710113545621246681116 789135685324561454691328101356812

91445653568169116123291012456816357625913171013689135134412331235156 83125261012468106910143158114134335714710131524616123

84112351232810146356544691456359106134291015235911513423123157102469 113134435912512361232567142466334614562789657954694346

\section{Instance 4:}

10102

106812395911414731236346547926681035810102910159789547107569813543466 56810123313411343414581232123410134189116123768127612325689345889104 79111123712358789158936789581110691075356124571241014562462123856710 75799123881015369116910141012311345569212341238567847812156987893689 2691165791061014

10910245946745892123612381341567314771342779104357861465910143568945 71057108245791014413479123656884671689104564123245692134757831346710 1584679245112341245791333681252457468144573357951236123441465568213 49123106123461015213491235789113481343681172451071015

10101057845686357713625793346981015879125147112324469578108156985685 14626784123934510456613591910135456847106457369139356434510569213447 3564346869126123121234891014191014661014712310959136246105698679478123 3457678145727811514787246214661354147113491231078103781045456156978 913891012

1098123413429101265812714636810109101411239691282678156775912101234134 61345910119710126345710781165787679113451343103569123846895125778999 1013156941231047102134313485710241469681068610122691253457781010357956 9195671934539789357105568

10457912413564571457813567457313510123246764564356978931081014867105 4710168101546356457103467258101025811612381347810143123913549101211235 567107812954579456867107345259131056715796891331344113489101537810657 86334695791356634510346868109146889101591256791343457691222463134104 69

103124556101283452513544686213484671091012156745811712310713419101335 7842466781191348145567921341012319910141991012101056825912746791344910 1284710691013513436101214561054694781299101118910105693567756784562478 6123221231047859671072464123145787910

10547101211238125635633571105671834594781254681345101237357224564710 93453569667893134108101573572589557101714781147259124246346871235356 989131013455579114585791056969101141134814561247456793577910141591159 1012691013858104245

10586811513410125713435811815688147101237456613451234791196795512394 79245745688123715693123101344569612357812714699591285671012371245710 1561232581119101131345459129671082451013465688656979101283574134213610 78103578535619136783571045691231567435666787134951341789456710810117 710133567913421346123 
101047810101367710152591361235610148581113579356356857346145781252123 63573712347898134621237134156891233134545668691144676610119910122357 101231059101177913991012134661231069118567379124123235651357245734574 6912913438134945723571661014137912

107624595681014541238134581012746792681284697891331341056794674691263 575678643453571087812712310578661013949101336895345779126123813410345 112598912336781579891012984564471024679810146246179125891131237468563 57924610246291013114752569959138356712357913457811913477812346921579 9147

\section{Instance 5:}

1582

106368987101365694910142468791015681347467512313456246378127513414710 361012858114571071456145758101421473681216710746881346135321233891241 34227810112568910112135658955674134345618671042125435655911779111356 9

14853462245314614794346679127479845667245613487812567845673710115613 41910152123545686910774694467158965678356335721341867107491013624555 792891281343710141134621347910116581117812459125345274673134657898245 21357245635642457567934571610126467758128781246913424684134645751343 23568457414648134445768912534576791313464681227893456568128345

1313123186812638910478112810148581171235781274456178116910123578547102 346713464245235681236245369101136571233123512321348134163463713411 45535638910112910157123126912654692457647103457445782458535725810457 10814613573468656775710551456456147871344135

128414781341478791011612336101121345467329101277913624586125558122134 1578891014713436811445616123465695134156921345412315676679757103345 157910878101113562781153454245691013313486910645693134671012212375678 3458145663465781286912213431347134434568910125456612342457568324511 579

12471233681081251691344246879126134334535145234545795312381342671041 256581226123212326123247966134881014134579101225675568585671567381013 661013789107457965812145751238123779102567481347134459111610153357947 10147456664573681044798891311345123

15751247678381013261012612345912114734134713434566834634787789213443 5664695556765691123778102134621231567867947812624571357847975895356 23456346313441254391013512341347456163578467108578313477810179112567 591014635768457145631235910116581047896789111789612342452356391011785 91161451710125468368112345734663123213675911513411368679166894267103 123847817910

15171475389116578512321247781236471041233671065568856738911658112345 71345735724796891053578691236710135345767821910146567821237678612341 341581152468671031235245635910156766795681023568159134491014567965812 856955891067912324684677781277910153591261348346259111356456761571074 7856783134678108479243467134

8241236134421234781282465578177897756855696581216912435626811847971 910145123368126910134567256785684612356781910128457521344691361341810 11813454123546961348134381015

924135245733125271011746911610137413686101525781245734651343610127413 4168102356513685673123735683134556915810812322466346414571341412318 13456467112338101251348467

124559118781242463135219101552454612336898681079101326123391014323465 125789131581013743453691024565910126691271341136378101231235245421238 3575134461015417811891012289113345464567710138346159124791012114531345 345

95489131791163565457867835910117569612373123545742467569114787912213 463357845661347467146922468813411233357434551236134245771247458112 123512431356134713485710185811214567345677910612353461147857103134 866124361014269101134757105345531344456278101610128134879101431244710 1415912678951238145224616567413452591185686569857810412325781589324 6645789101371348778925910179135569445785673910136245388101571344123 
1482135812376791569413631476134513428579579114469117145812316792447 1014566612323573789424574698134181231134647356135687810591012731342 13411347710134671051238123521345567635711238357423463789647105610158 2136712415811512344710613631348124515811734645810567861368878971236 56855693678414725671124

10273565134655678345758112356635737810341345810142134426812869115125 4710158568128789724562452346335746791456433455569624615691656913569 75591211238671071233134656825797113471232568681012356854564678 1044678612474675123451237134812441347113451237123878114467214663462 681011834588681045910579102568361014778961341136364672610128468753466 610127791316812845637810212368681071342145589114581033458113421346123 724688101155683356469106661011112337812413484675123

\section{Instance 6:}

15102

1368678112355693134234679101510108101113578346981013679135781275812446 92134381015761343678101455567256911348781059791034578910134345689123 107899246347106791011591013835691342910154810136812327894479959113123 107912375784245101463681015512321345958123134101342678634610735795788 67106679567918101537811104674589258111313456123991014135737812781015 1563910137910159357814610123571014811353246814710245614599101141345478 8106897145513435796245135623569568754783125778129468624685784124881 23561013113571236457956843562681062145868115357167810134713696910132 13454671059109146145644563357857101912397810118123512321231013464691 569324541233912311458478574710113485792910136134249101291248867102710 129456102453456724513465456879101147101396811556761348356124635911181 23

10695685124145610123445781342645614781035899123458121057822451357814 6769106123534644134156895896346323577123134631057951233469110345235 8119356625710661013157999101157101271234105699456178927911

132657891347289104710125123771011668997891591159457713483464910125710 149567101134968117123103573357613449101287896613411237579935686101210 1235912344710857103123713459123212385675467656818123671255134947103 3576478105681513435134845615710963458591223563568914510145713415695 3456312311256134213454578356

10923465123956973573610151078126356813441237569112457889133345147968 91245677678101012317101125912945653454345734564135647873459578535682 465361014812351346145178111978123213488911481015835691581287912924525 912768910671041347571015478111479335764787123102468578103789824697810 62454456101231467

121981012784676681148101324795891175693591221046762456546875699356256 710457161015415710945782467591017471016345243455891054710157123159116 5892245265699781013581115246

134913417913413479101179346881013778122123101236567112335791127912767 831059116568258105668997811391014412310134375675681085681535634791337 811289118867102589567810679669113146412497810541231012371478135635610 65698356345773571356513424579581041341012392134834667101471231671091 24104794891154577956756101561237610158346379104579

1321024692464456935910891013146823345135734569913486785156765684346 10134935679457389114346245783577345578117102451357491011612395792134 889115213447913312355710961015141237759129891110671018910512363464245 11012311091012725913101239567714769101217810881011

10410147812331345134551348781036810101349345447812835765710913418135 22568101341412321345657821024693466891014313457912778124457957823134 10468

1022123106811110567105357813446811371015789129781019101567892356101349 95694456691011534510123835679101235672125871348457635797811547825674 479324651810129124734631346147896101311347467835754710261013478121046 9361239346834568123212374783246113491247235610134813479101565694345 9134 
13108671021231456924533575134645610789446774562958126569992466246856 8108101218910513436101276913224564789545633567791010581067810775911556 781341357661012412327810610781092461589712345696910132557871232259108 56759456768108346259113124484784123612394574971012324624577791131134 10123967810224563575681010581243568910157345334595781135

157124533566781010568956986812756727356247941124691011834591233271013 10123345638145213466784313486101110246789131524531012321238246614799 1344468268910610115610119713493563910135135878910571027101469101411237 712326783346101235124812317910397912881013158112835610678874671012386 89135641345469612536911564797789213415678124

14111453101348691244699113455672123457106456312385781067107123226710 1679735689345524571352345614641345156793454791326910735759781011234 45679101331236645710578889122457767946911142458591015634683564134314 6147101045691235569104478947102124104574161012779131069139123510246113 48457913456911243463134

146113425812812398913578106246766795469934510245735624571810136678113 12348101272469245513558691013459134768935812105356257868913446993468 47101059103691211247345841469781021348245107912712355673456921235567 947107346105911178128123347862457212310134414572451457979136567396913 25710889136313421231691371346910125246845696134712334562710128567113 496710981242123513437811158114678989131056773561034568581010568724618 10132123556741479910136457421239147691013105710

105524683469781277810234621069103136210457191015581241671051347781237 8104359107457914668101591091013112361457781225913824646793610145346773 466134113443565356361013107913951237691087101262462478445735671013491 2369123867916101277811261015107810897101451231078124246212477910334668 910

\section{Instance 7:}

20102

1789123646735691024574794357814553571556710414710123878127579634656 101296710378122145135668791075691013417810671011946961568968113134512 564687810143224661239145691237568869104147247101013412123987810991012 712357101215912379134134107912212458689291013102457457635710613455698 7910369104810159781176101410356212314791157810191014979132791384574134 34791012354567456624654579535782452591210357210356312362591386894123 71346568135631024525693679

197647893565781210581213577910148123174682778104135221256691127610129 791277567381015512310123869112910114134871349681210691215785134356747 81161241513486579181011914727912467101012372463346961348123414631349 356101235569261015713655468758986812214745811331232810128136677912214 796912612314575681121013493462791013524696134971012712341248468101231 457312326101131058102123624663357913482454357278106123117811

13727812491012371013659101078108581253461912383691096101586912414571346 356557101012333568512310678874571810138134512341341013431232456108710 12447106478113493561056721343134513471238668111068108457456751231246 21233123885787123547991231356312545681012322910143810141145751134107 10136134478118591214478241353134

143645776710824598134446769101232457581151361013497811259121213445568 91342123334610103451456334577101593466346447857892145868910213495911 6134713441463891088913568101061014191015310345146884798481015178107134 1079126581158912968113568759101517812869109910126134424531251027896123 95675123871011312345671067918913758961058123356156861458456212351014 631258147247991257104786134812321343456514773574513410681134788134 1787810126910141012315911945653464123212394134535610479659117710151345 8123912321369612595911724581341012444563891021345457381232245105697 914717101185811746758911102466791097571010457513446788457324511346610 139610141057811734581251579671013913442452569102453145491347469101232 58113456871475671092569858963451246745755913413431361056810259137123 81341078111136912366785246335641347613434797134867815710106913413422 
67841232378910134689101241345589102467123261015831344789512395810813 5114761467691267245513484676671041359134

1437810119710138346791452245471014734614576123557893123106101484710756 74910112245957914575471022134478126234637897245179109356512397569278 98345912365893245105674134578113713461239581028478281011551231123212 36581231342912319101527568156967357414793462579368115123719101361349 67878101559101346810846786134178118679981013281013534534797357

204769126134101239134476811102454671087810726910912362451578856956910 789128389127346412365672810115469101349134687911261012512376810669114 1235624631235781275913813494357735631342357634591345467812310791145 123978910781274569103455136871012291011612311239134778941365356810123 91472789647949245102463457291012265698691341479957108456758102112381 349334698910491015861012712510567647951452245588101135810512321341456 87567924631465123661015234610781042451524688569967104781110345156828 101159101474576513410910132581171236810148134

11196810192455956815710435774561056782567835643576346771013105691679 378111105675758951344791098101167911104568961011369137245679121357859 1326913101235123108478745710134913417101259101445673345267106469887910 547106791235697134913412461091013773566569568124469104571610138134361 3611349123

12235681147841237781283576124156992451078113891081146959126357712384 57513446101234579845793565346312415691012345910713425810821238134969 10112363575689456935710782451013421237357913442456135109456635686811 106797679513441233456279131691091246914566912813479101337812434610134 23453813451232123552452781110123958971344614647810181014712359689446 71012371245134

127234694674681276101264673610115457435781068102123612324681115672101 23712341134457928912735655810159678367923468134888912935751237791110 910154123212335681047910914783572569724668913578103681211251056946147 94683610127134711232591337811412359101391347123344689356624577681069 101111474591210245267936811

1567134378118123545710134471012353467571017812108134769119467101456910 124246513434572691316912127101265578212364561045786101246911741475356 813431342134178111069106613491343810117468224658913617101482453691123 56634595677412362465134107101318101571453456646811101348569578961232 581131056791233134528101491464810151012371246245681476710119246745641 3448136145765812107812610245156737812735791348469

15726781123868107134104565123981011555681068128134224544574756941458 47927913465695124713416101246134924681343891283471015688467456910246 6568291014713625671097101235246212318101151710142569101233467812381068 122581154710756946101398912379101123545785356313471349246103134556883 469125468101134109101165913278117246372458591114566978126781082453610 135471010789655679145105693134634585710

1831135567986812271248123101056849101416101266101327913513475811879133 79109568561239345881012146851342968128710154367971345567124538346934 610356747101185689456756963455591125693561013101348135971344691053456 5811934525781035737101314571412376810117910159457335610134578102678910 4710514731239245612321344123846818101584123612310123734683573345912 35710119412381259710115581036913713565781078112910139512381341047102910 126246312441237710149134491237610151456412554571010123812391235357 113335791361134825912147108591341236789559131071013378113412432468123 1524571691310579634527101544575124791013739101476911145721234910128568 512414456885911124667911558978101297912101243134789101466911103571246 445673465679186101475571015686134947810123414575911

20691341245471013812321231059135214791238679578910469123561112344134 513417811246946134912415911445728591123451028910971011156941231013551 47312366911891011712310513614788457979104679289123456747810568656967 1231013421349135668124134107571095788610125591064781091011371014167941 34212368581091344246212410789778121031349591255684123246771471134889 101035762451512365610121035695913224532454710117924535784710145567656 97891285678889132246412315677471051343134946856123278118478105710345 67813624685567791013614537101110345710910144357724518910646834575134 
2053356813461474345107811493454147156931236856931476589424615685134 15146576101466795346913410356592466781123454123101239378991354781267 8112671057810156810581285913827101356810856810789613417101375794346923 46856797812424510146556711233145645751068932458610135246656810678910 24524561469324551474124924586101476811310678247105568693578910154134 10123656831348545694573246878127471025810446710357910245512326812767 89245356784574591311349779121013451231146413431232791181349681112125 927899467434577101410456656935785145191014710910135578156996894134812 361233513493451568

158245619101195678910133123524610679459111913627610154357272451123964 57346810681292468568713448101351231356881249134514721236123757104457 3679775789134846934575681065691059112467925910526101139101419101510468 9123885811481011614671349910115357105912381011654781046869101474710978 1024784912510781157811679107579119246105910134539101289101144572102457 2465489132568101341910118789

1824123105691054569478835765912358101061012157102357413471352835646911 52569313410891091236567810345434687812768103124289136891391345156881 23312347810661014527811957933451013441231378982791071234134105698134 6891239101496811265682134329101297810561013875910456910123956865785246 124631354713411233471085684212349101319101177101394579246774569791167 1015367851351068986910245671135972469356659101246108913567104781235811 2910118246844578346745611343469557109578

101051458681245693136612327101375913101341910129135231345567645699123 2123113510610145781111013459146413467101518913757101534518246846710107 89867913566124589127134271013210246956736123845617812

17354569346412310961014245655691034645677591281476681034571123937895 1346567812344781012411237791122456646951238567756810123213464469534 684566791211341045773610157123612326910945646913135764591396912312371 2357911656855679913473462246824648345312321345678416913101343246846 958135534597911168107134291464710139108913912439101475710635781235467 45782134109569881014534641352123368127679105811624517812124568934611 463145868106610134134212374710156913

\section{Instance 8:}

20152

20111345121059115681083561312414683681015123456773456910111412311591367 1231013581343781212134556712379102891315671178101547912681291231413574 781334552466457387812123561123111114523561412312456245847915357101239 781112891155794156793246469111213414112473455123125671112310678356794 561467986792134489126456131341537101310589213412134154677134813411134 54791412361344579979111457131343124551241435797478123451335723451178 9102453810144478153562105912668101413134812578913147911578151344589212 46457143575245101233346127101335910111367849101361212311134768124123148 9121347912247812589634613456914611246155811558128134334517912141471212 791213123158911145891112336799345846761341056951234569984569910127479 59101511123135671810122456378112389138456283457136

176447105910141561014756781342123911136138911157812312310910125781297811 445667101529356756859357724615123146711791110146714123111231259131579 1335899346131347146469111424561056813456867856101115245757101435611135 1123121234781037811657813112458781012781134681089109589791013144784579 624515134545713467485781212315123345758457645636101412781095913141136 8123124569789115910132453781151351534610134613446913713425671336101164 564567124781157975811912581341524513136245714679513453134109101386810 7345657913291012155912314510123557971231471014656881344610131313512134 913481312375699810146591112134356811465891013111232145746812569946913 479312310456147810556841341547106134151112551351545648913144566123935 7101348246135671569724531342245121341326910147101212134155710103571124 51568813413456614755910413496710

15657910144691012364710111471234631178910710111335751256726912712310810 12111347913466811145911746831341571015478310610141112343561481361478915 134947961241178115134123577134258101034543451334533451066710835711123 
1235610147214715811134679346513415158101210136127810924613246313451368 5676610132791111123147894591114567710135945631231313419101383451196810 1446931351281014878122456681011158910105691691251231496794591362462123 734583571414713135578111124631341067815357135653134141347246571015101 34885912134671267845811119101394681068101545718891310259101256931346357 54571178991231559117579106912

171415589133458581171451478101349134115674345125795457145676356367108 39101513569534610246148910612311471015781021123735615961013612337810156 81014123268127245125910412313910128568116101153451089121681012747810610 115678121341135664579569813413123151233691147911513567835743561581012 1191015810457468111512312357111366791111239123254698579773454123191013 13681011791286812912371134101341313476910568112781014123636101396101210 13416101127811756921512310691053345147811524667810131235989121578114710 15713511791115121341471014891271461324625681561011371012912311136581012 105911445761348246126710151791271345125127811457815910136457131349346 114568568146811391015256910568

153712314123113462151241479106158911149101279101599101310789545647568145 8121345761341215695478812310610141445695671335711891012681271233123646 958134478117123178111146712105681256913567141361112315788246414675696 78105579213410113415567133461014778912867106568481011126811345791213414 146152452467115674357157997910767978681257101113134113452789152459246 11791012145812524597811151231113467101312581031231123107911451461414710 457127897102451478105246125691356915246935653134153451357126797567315 134146101112123

20113910153978966101313123715357935771458134124710279101791132691335811 791013951351424583561024511245151357134356712346821341013413571093568 134127897679124595581214891144671267911123105710669117356369118746897 895245259101781212134156798691213113452134135787136101348456356912454 56914478121231567955681128101214391011413415456136796567714511689835710 47812781252461445611242681181256991342710153710141078121389115471014356 10678111061013151474791011345145797891457878101186101415912514356747912 6101213134104576610128123157811213647812369115346115911113414103575789 1546771241213426799471081241581111123134683468612314910141098101214810 1213134115688610126456556715671077101136710112567344571034613467114123 1059103147121241513414568913425812133566356713412589111012313345614514 12315134313427812124710724593568147

17615811479121312312123971012578122134781078118144671213413123468129123 1013456792571021545714123141591013141341313421453691244576123125677134 1356912385675791011569515691033574457125913108911133357148913113412123 2457116710135698245713495686910124246524613967814123312311134691015153 561267101058112567135710558941231679351341057961241481342781265812413 4111461024514457956713571513452457910113791313910151156812931341567812 46947875671313485911546714123111445715245137811978107246105912118101362 46861013478101212325134154561151231034512134947101391015846714571044710 76811245718910715698710124591315910141112433561256914658914781181239910 14558111047104910111113412479247912467134151231381014

18111569133577145122461134692461514561341413483575123151451221458569 1545611231124610125434679101112579142469123134567111231781110123153465 679745794571011254123148910151351179133791364571335684797569127679141 2411568913411241571014134578147121345147104564567711781213681227910724 6112361259789631251367101134212557812413491391011104561445612581111579 5134913434691691115154781447105581187101595672681134686567101344581175 78132451213411568189121291013116591385811143571256791347610131169123456 1471013781210125141035714468513491231356115671312333466581015346445686 781212324571514681123561123812354794123659137346111231345615910131278 12359109146101341321231512387101332455678689117678139101111591314356128 10114478913416134141471015156710113429101310681161471213492454689135910 112457124367851341511123113410681051451212414134413485710712313246356 721239345678915345

197145614891011134459107356558109345611356121347124147812924652466847 85691028910647101212313710151526911111464568141349134813454671212375710 13134108910371011671013156815346136671073561268101135739101191231446741 
23212413123589121512310781221467912571010612451231235710357136812256715 1343578145911858951391013101231469125685346712578145710247813581215468 39101411341091232123324512469154671471013558101045611781013781151781027 911131231258121161011101156794686571073463581214134137891078111291013413 415712315679112334695457846894682467413413791310791011781161231435712 6911142791241457245114681413413134924589101415910151014765795134121343 357151234518912612313457214741231035715781114245111235135912335688145 734511125101589153564469131341113491471461014514712781262462413410134 4747915579135682134

1610359111471014105910912341342246131348710141213465676112544673679121 34889122134811910148689556812681115781210591127101493561221233134113412 1349468458115135151231378108681161341114525356136810216101235681587810 1123561013121231079134457115911144781312375786123312315246256895781321 3445913834561239567324512679756714810115579119101310345147107567914123 12691210124171013958116123712678512513468123158101271251069118116710147 810157101475683246107891279101910151177812691014142461113491231045715123 1361014213681235346314686457458121565812149101154571312385674124356912 67109467767816810104791159111591012213484123157810106810224511134113413 479347937134913415134

204589118591262454134141134615910119123133563568101232468713481256134 491011545614245122451131341053571456741341068913561135793462123646984 57111157912910148245131231561014659139345101345581014134467871367865911 15123561014269121035694710594561458115345712341237213415710581012126911 15781184579910112768129781014268111456514612610144571010134116811656713 68117567154699478146101485710315910121781111134138134135633571579131245 74781113124234514467712395785791362462145757811811135713435688456145 891578956710135794934516101282466789126787857914469135676567157912578 93561435615103571445713671093576356119101112123167956913356715123491011 779128567278111210910116689335621231145685791312312679779139578524548 1011

1934457535612710157612377811132455123121451679118913135134971011141238 147156784134101231289112123334572451359136691212534543451213414135113 42357978913124612373461189123123356912268108567153456647915246956711 2314681111246133567357861012512349101310123121462123695911141257578156 91211781135710118147156813781112146334515810141112521236357769121068914 101367134135689568535611451158121458101512346786125121453467813461559 1182452568678114781010691177567568935911146791312311134634513612313134 812352461113410245178113781223451512396812127912143567107898567113465 67116792781279101153245156784456124710556712155812116895681127812645614 89123610128123778910134412394578646714781113691215568119101597810712410 59121295681524517891267101191015781013141341024556101361238781213679567 10141056812591115781216910137891013357413491256246104671279118591115579 1112314345359105245

1713335714134579139791311237568646715610111313684781234642451113432569 1224513136319101214568123575145615125759105467104794913411478146811121 461356787456151236345291011106781259138145414691341424632451324638567 1456861236213615671067101224611245991012127346141341512312147117810824 69245481015105911157102810133357131258109246112421238136312415134691015 149101456101210357413413356151069111157101212514147457109134735719101315 91012135692910116710133569579128123642459123258121512556781456810116913 61479134356829101110479759111134131341412313127101185913111341089117134 556895810647101414523571534541251345710624514145712321235134151344134 13468989111012311141351289128478101231179101610124910129246155676781056 101413361014112455581126910145678246914566797569131344134105811125912 191310245535613134661012334511349568845712134113567146412315134132569 1124514356124791391014105699123312377811845667101443561534681781015134 12123106791171012813437911681012791238469313413461146810345756813581011 12681124784135117812144691591014861011789106345101231335734710118123148 91013568391011115699478124791557910610111891263461563455471037913713415 24695912149101585672467128101141341068111113418101313123351241424635683 7134111341591191059111459111335641347134991013153451213411689109245412 3155812279111113414471051231346101238469148101371123121341371012935676 
8101591013335655456122451346247946710783451035713457514619101114478129 10141512457389131891014791154691013461348134712342461524626891181012136 81297910213456105891512671011810141345611471067856710413491231413477912 8710121534624693123624521469123124

1712346714123456871471324515679128911989116457213459101510691063791141 236123556891341513496568412385791123115681561011513475689581110153565 123136810117893345645691238456121461058915124671471256994791359122136 8135569123479668101024543451467101159101513410712513134845657101510910 1111568912326101343571213413812591236579151342346378107679111341446712 4685791241351391014510791284672134117810557107213411134141341235634695 1231056761456133451024571232910145791076124124671134151233135881013104 5615756813123125910391014113561045686810689101434515245567991344891314 5726893213461461313411212310105676591013571081349357153563589142452710 117467351231212314469718101128101361235791211136735712245

1945357679128571012681010101238123456735812125695246142461467668107457 13169121413415591272456578212313781211245103578356535612910143134918912 56910991012151341068118671046781267102134215345346810256810356134561556 717893810149569513414457656811978926787591312123111468457104683145156 9111313451358147811524611246125691345312347896569567811959101446927812 13357713910151156874781281015144783356106786424513610117145155691178931 34541343457107912912312123411689513414710111213412106910141461279101157 95579334581341389102479669111457435714656735671324521341113410146834 51534694561424557812121357123124514159101214567769139678108101259101212 123813449101411569613513346368102357915591276781589235713147145710957 91258965913243561245375671256815579

1915148101258913151238134913410681161341334576812111231134123462679412 3347931512314781124781313679613411469259101567101012512710149568357972 45524614591048913111581015135613789371014813411710149146545712679756925 7921035712478121341524561267864561456796913108913312415345761014134710 891012414618101158910112458115675136141471313410345445685676134615357 1147822453691191341413691012315591343572123581014861015146913134567123 71581012745614781012478212313134156714157863451112312610127810141012385 68152451345614134578123357481014912351568111012461231434611810151141255 12689153455356117913345761156915245513461342591312346414123135810127811 13565191011116811135671267102910115312314689667812610152245

161151231478111278117479112313891210910148571015567658937101510125710147 913858915478104564123912311571022457123636912141231045743455456136911 141146147912945612467612311357151231357853567610133123812341232123153 91013119101191237581114123105910814669101312134151341810134123214513467 5245211581147811121514669101211232781113123121239457116797569813414910 1445793168912135613412359131181015813422459578546915910151378104134612 312467105691366710126811147913981011116891047813123157912356971345910151 5674691051123513415478114571368951278914781063579789101341115479101344 3577781211581235781424587811945761341312413513414891113581143573567157 891012311348571095911635671241135763357813413591214457959101245681567 8101231467931237569468121113485910

181414134478101179121291015978977101385791781261231357101047815810123124 591015224796134915894346212435910145891334556897134121231023561413413 346122451035611349135614711791351349368128356146911113423461035611134 912372466151471371012813412469357591013813145121456147213694710101235 35715357245811991011124147556812146146710912417101162468610121313410346 334615679156123113451545714578845751341356994694123107812121342123345 71581079101223591011810115979114710151781110245257895591394687691112678 1159108124151341314614346396710647102578121213433461345614346969101013 4157810413486710214753561168101103451276910132469246147108246569111512 311246123466123214531347814547810667101546713123179132123

1713147910778114345313411479101231313412123113465688791322469134121214 715910128123246911134105710956733561424561341146413415234610123713412 3571135144561135685810312399101155912151361324546796691173456959121056 813568155913146781781146581115811134784135935897134513461231312310357 1545611474571015123111158101461012131347789867891231259116123113515123 42456125681081014155912312314910151391013731232134104565134114698346912 
32156912245114245271015191013126913113561413515581093577134691011546782 456141231571013118912179121056956101135912921461335617101555794910151014 631238910111246715101234579813466891135141239581013457534531231545612 245112452810117469985811151231469109910131167101045775913291012467101344 791459115457691015121342569924585897891215581114791012311579 